\title{
APPLICATION OF SPECTRAL ACCELERATION FORWARD-BACKWARD METHOD FOR PROPAGATION OVER TERRAIN
}

\author{
A THESIS \\ SUBMITTED TO THE DEPARTMENT OF ELECTRICAL AND \\ ELECTRONICS ENGINEERING \\ AND THE INSTITUTE OF ENGINEERING AND SCIENCES \\ OF BILKENT UNIVERSITY \\ IN PARTIAL FULFILLMENT OF THE REQUIREMENTS \\ FOR THE DEGREE OF \\ MASTER OF SCIENCE
}

By

Celal Alp Tunç

September 2003 
I certify that I have read this thesis and that in my opinion it is fully adequate, in scope and in quality, as a thesis for the degree of Master of Science.

Prof. Dr. Ayhan Altıntaş (Supervisor)

I certify that I have read this thesis and that in my opinion it is fully adequate, in scope and in quality, as a thesis for the degree of Master of Science.

Asst. Prof. Dr. Vakur B. Ertürk (Co-supervisor)

I certify that I have read this thesis and that in my opinion it is fully adequate, in scope and in quality, as a thesis for the degree of Master of Science.

Prof. Dr. Hayrettin Köymen

I certify that I have read this thesis and that in my opinion it is fully adequate, in scope and in quality, as a thesis for the degree of Master of Science.

Prof. Dr. Mustafa Kuzuoğlu

Approved for the Institute of Engineering and Sciences:

Prof. Dr. Mehmet Baray

Director of Institute of Engineering and Sciences 


\title{
ABSTRACT
}

\section{APPLICATION OF SPECTRAL ACCELERATION FORWARD-BACKWARD METHOD FOR PROPAGATION OVER TERRAIN}

\author{
Celal Alp Tunç \\ M.S. in Electrical and Electronics Engineering \\ Supervisor: Prof. Dr. Ayhan Altıntaş \\ September 2003
}

Mobile radio planning requires the accurate prediction of electromagnetic field strengths over large terrain profiles. However, numerical methods, like MoM, become not suitable for electrically large surfaces, because of the $O\left(N^{3}\right)$ computational cost due to the large number of surface unknowns $N$. The ForwardBackward Method (FBM) is a stationary iterative technique for solving linear equation systems resulting from electromagnetic rough surface scattering problems and provides accurate results within very few iterations, causing a computational cost of $O\left(N^{2}\right)$. The Spectral Acceleration technique reduces the computational cost and memory requirements of the FBM to $O(N)$, so that the Spectrally Accelerated Forward-Backward Method (FBSA) can be applied over very large terrain profiles. Empirical models with reflection and multiple diffraction (RMD) corrections are commonly used to predict the field strengths over terrain profiles. In this work, applications of the FBM and FBSA are presented over electrically large terrain profiles. Also, using FBSA as a reference solution, the most common empirical models with RMD correction methods are examined to find out the best propagation models. 
Keywords: Rough Surface Scattering, Method of Moments, Forward-Backward Method, Spectral Acceleration, Radio Propagation, Propagation Models. 


\title{
SPEKTRAL HIZLANDIRILMIŞ İLERİ-GERI YÖNTEMI İLE ARAZI KESITLERINDE DALGA YAYINIMI UYGULAMALARI
}

\author{
Celal Alp Tunç \\ Elektrik ve Elektronik Mühendisliği Bölümü Yüksek Lisans \\ Tez Yöneticisi: Prof. Dr. Ayhan Altıntaş \\ Eylül 2003
}

Telsiz frekans planlaması, elektriksel olarak çok geniş arazi kesitleri üzerinde elektromanyetik alan şiddeti değerlerinin doğru tahminini gerektirir. Moment Metodu gibi nümerik yöntemler, $N$ yüzey bilinmeyeni için $O\left(N^{3}\right)$ düzeyinde bir hesaplama maliyeti meydana getirdiklerinden, elektriksel çok geniş yüzeyler için uygun değillerdir. İleri-Geri Yöntemi (IGY), pürüzlü yüzeylerden elektromanyetik saçılım problemlerinin sonucu olan doğrusal denklem sistemlerini çözebilen tekrarlamalı bir tekniktir ve $O\left(N^{2}\right)$ düzeyinde bir hesaplama maliyetine sebep olur. Spektral Hızlandırma tekniği, IGY'nin hesaplama maliyetini ve hafiza gereksinimini $O(N)$ düzeyine indirmektedir, bu yüzden Spektral Hızlandırılmış İleri-Geri Yöntemi (SHIG) çok geniş ölçekli arazi kesitleri için uygulanabilir. Empirik modeller ile, yansıma ve çoklu kırınım kaybı düzeltmeleri, arazi kesitleri üzerinde alan şiddeti tahminleri için sıklıkla kullanılmaktadır. Bu çalışmada, elektriksel geniş arazi kesitleri için IGY ve SHIG uygulamaları sunulmuştur. Ayrıca, SHIG referans çözüm olarak düşünülmüş ve en çok kullanılan empirik modellerle karşılaştırmalar yapılmıştır. 
Anahtar kelimeler: Pürüzlü Yüzeylerden Saçılım, Moment Metodu, İleriGeri Yöntemi, Spektral Hızlandırma, Dalga Yayınımı, Yayınım Modelleri 


\section{ACKNOWLEDGMENTS}

I gratefully thank my supervisors Prof. Ayhan Altintas and Asst. Prof. Vakur B. Ertürk, for their suggestions, supervision, and guidance throughout the development of this thesis.

I would also like to thank Prof. Hayrettin Köymen, and Prof. Mustafa Kuzuoğlu, the members of my jury, for reading and commenting on the thesis.

It is a pleasure to express my special thanks to Dr. Satılmış Topçu, and Prof. Hayrettin Köymen, also for supplying significant resources for the development of this thesis at Communication and Spectrum Management Research Center (ISYAM).

I would also like to express my deepest gratitude to Prof. Levent Gürel for encouraging me for the graduate study on electromagnetics.

Finally, I would like to thank the Spectrum Room people at ISYAM, Burak, Gökhan, Sarper and my dear friend Alper for their cooperation and friendship. Let the celebrations begin. 


\section{Contents}

1 INTRODUCTION 1

1.1 One-Dimensional Rough Surface Scattering Problem . . . . . . . . 2

1.2 Propagation Prediction Approaches . . . . . . . . . . . . 3

1.3 Integral Equation Based Methods for Terrain Propagation . . . . 5

2 FORWARD-BACKWARD METHOD 8

2.1 Integral and Matrix Equations for the Forward-Backward Method 10

2.1.1 EFIE Formulation for Horizontal Polarized Incidence on Non-PEC Surfaces . . . . . . . . . . . . . . . 11

2.1.2 MFIE Formulation for Vertical Polarized Incidence on Non-PEC Surfaces . . . . . . . . . . . . . . . 17

2.2 The Formulation of the Forward-Backward Method . . . . . . . . 21

2.3 Numerical Results for the FBM . . . . . . . . . . . . . . . 24

2.3.1 Applications of the FBM over Strip Profiles . . . . . . . 25

2.3.2 Applications of the FBM over Rough Surface Profiles . . . 38 
3.1 The FBSA for Quasi-Planar Rough Surfaces . . . . . . . . . . 57

3.1.1 Spectral Acceleration of the FBM for Horizontal Polarization 58

3.1.2 Spectral Acceleration of the FBM for Vertical Polarization 61

3.1.3 The Deformed Contour of Integration . . . . . . . . . . 63

3.1 .4 Step of Integration . . . . . . . . . . . . . . 67

3.1.5 The Computational Cost of the FBSA . . . . . . . . 68

3.2 The FBSA for Terrain Profiles . . . . . . . . . . . . . . . . 69

3.2.1 The Deformed Contour of Integration . . . . . . . . . 70

3.2 .2 Step of Integration . . . . . . . . . . . . . . . 73

3.2.3 Computation of the Scattered Field with Spectral Acceleration .......................... 75

3.3 Numerical Results for the FBSA . . . . . . . . . . . . . 77

3.3.1 Applications of the FBSA over Quasi-Planar Rough Surfaces 78

3.3.2 Applications of the FBSA over Terrain Profiles . . . . . . . 91

4 COMPARISONS OF PROPAGATION MODELS WITH FBSA $\begin{array}{ll}\text { SOLUTIONS } & 106\end{array}$

4.1 Empirical Propagation Models . . . . . . . . . . . . . . . 107

4.1.1 The Okumura-Hata Model . . . . . . . . . . . . . . 108

4.1.2 International Telecommunication Union Recommendations 110 
4.1.3 Federal Communications Commission Curves . . . . . . . . 113

4.2 Multiple Diffraction Corrections . . . . . . . . . . . . . . . 114

4.3 Numerical Results . . . . . . . . . . . . . . . . . . . . . 117

5 CONCLUSIONS

A Spectral Acceleration for the Backward Propagation

A.1 Horizontal Polarization . . . . . . . . . . . . . . . . . 170

A.2 Vertical Polarization . . . . . . . . . . . . . . 172 


\section{List of Figures}

2.1 Problem Geometry . . . . . . . . . . . . . . . . . . . 10

2.2 Surface Discretization . . . . . . . . . . . . . . . . . . 13

2.3 Forward and backward regions for the $n$th matching point . . . . 21

2.4 One-dimensional strip profile . . . . . . . . . . . . . . 25

2.5 Strip profile excited by a grazing incident plane wave . . . . . . 26

2.6 Induced current on a $50 \lambda$ strip (TM Pol.) . . . . . . . . . . . . 27

2.7 Residual and Absolute error for a 50 $\lambda$ strip (TM Pol.) . . . . . . . 27

2.8 Induced Current on a strip (TE Pol.) . . . . . . . . . . . . 28

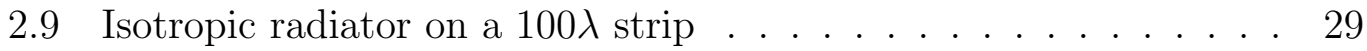

2.10 Infinitesimal Dipole on a $200 \lambda$ strip . . . . . . . . . . . . . . 31

2.11 Induced current on a $100 \lambda$ strip (TM Pol.) . . . . . . . . . . . . 32

2.12 Residual and Absolute error for a 100ג strip (TM Pol.) . . . . . . 32

2.13 Induced current on a $200 \lambda$ strip (TM Pol.) . . . . . . . . . . . . 34

2.14 Residual and Absolute error for a 200ג strip (TM Pol.) . . . . . . 34 
2.15 Induced current on a $500 \lambda$ strip (TM Pol.) . . . . . . . . . . . 35

2.16 Induced current on a $1000 \lambda$ strip (TM Pol.) . . . . . . . . . . . . 35

2.17 Induced current on a $2000 \lambda$ strip (TM Pol.) . . . . . . . . . . . 36

2.18 Computational Cost . . . . . . . . . . . . . . 37

2.19 Induced current on a $50 \lambda$ rough surface . . . . . . . . . . . . 39

2.20 Induced current on a $50 \lambda$ rough surface . . . . . . . . . . . . 40

2.21 Residual and Absolute error for a $50 \lambda$ rough surface . . . . . . . . 40

2.22 Induced current on a $100 \lambda$ rough surface . . . . . . . . . . . . 41

2.23 Induced current on a $100 \lambda$ rough surface . . . . . . . . . . . . . 43

2.24 Residual and Absolute error for a $100 \lambda$ rough surface . . . . . . . 43

2.25 Induced current on a $200 \lambda$ rough surface . . . . . . . . . . . . 45

2.26 Induced current on a $200 \lambda$ rough surface . . . . . . . . . . . . . 46

2.27 Residual and Absolute error for a 200 $\lambda$ rough surface . . . . . . . 46

2.28 Induced current on a $500 \lambda$ rough surface . . . . . . . . . . . 47

2.29 Induced current on a $1000 \lambda$ rough surface . . . . . . . . . . . 48

2.30 Induced current on a $2000 \lambda$ rough surface . . . . . . . . . . . . 49

2.31 Computational Cost . . . . . . . . . . . . . 50

2.32 Total Field at $h=1.8 \mathrm{~m}$ on a $2000 \lambda$ rough surface . . . . . . . . 52

2.33 Induced current on a $150 \lambda$ rough surface . . . . . . . . . . . . . 53

2.34 Residual and absolute error . . . . . . . . . . . . . . . . 54 
3.1 1-D finite rough surface profile. . . . . . . . . . . . 58

3.2 Integration path in complex space. . . . . . . . . . . . . . 61

3.3 The original and deformed contours of integration. . . . . . . . . 65

3.4 Integrand along the SDP of a flat surface. . . . . . . . . . . 66

3.5 A generic terrain profile. . . . . . . . . . . . . 70

3.6 Integration path in the complex space. . . . . . . . . . . . 71

3.7 A generic terrain profile and scattering zone. . . . . . . . . . . 76

3.8 Infinitesimal Dipole on a $200 \lambda$ strip . . . . . . . . . . . . . . 78

3.9 Induced current on a $200 \lambda$ strip and absolute error (TM) . . . . . 79

3.10 Scattered field from a $200 \lambda$ rough surface . . . . . . . . . . . . 80

3.11 Absolute error for 200 $\lambda$ quasi-planar surface - FBSA vs MoM . . . 81

3.12 Scattered field from a $500 \lambda$ rough surface . . . . . . . . . . 84

3.13 Absolute error for 500 $\lambda$ quasi-planar surface - FBSA vs FBM . . . 84

3.14 Scattered field from a $1000 \lambda$ rough surface . . . . . . . . . . 85

3.15 Absolute error for 1000 $\lambda$ QP surface - FBSA vs FBM . . . . . . 85

3.16 Scattered field from a $5000 \lambda$ rough surface . . . . . . . . . . 86

3.17 Computational Cost . . . . . . . . . . . . . . 87

3.18 Induced Current on a $200 \lambda$ rough surface . . . . . . . . . . . . 90

3.19 Induced Current on a $500 \lambda$ rough surface . . . . . . . . . . . . . 91

3.20 Deformed contour for a $200 \lambda$ terrain profile . . . . . . . . . . . 93 
3.21 Scattered field from a $200 \lambda$ width terrain . . . . . . . . . . . 93

3.22 Absolute error for $200 \lambda$ terrain - FBSA vs MoM . . . . . . . . . 94

3.23 Deformed contour for a $500 \lambda$ terrain profile . . . . . . . . . 95

3.24 Scattered field from a $500 \lambda$ width terrain . . . . . . . . . . 95

3.25 Deformed contour for a $1000 \lambda$ terrain profile . . . . . . . . . 96

3.26 Scattered field from a $1000 \lambda$ width terrain . . . . . . . . . 96

3.27 Absolute error for terrain profiles - FBSA vs FBM . . . . . . . . . 97

3.28 Deformed contour for a $2000 \lambda$ terrain profile . . . . . . . . . . . 98

3.29 Scattered field from a $2000 \lambda$ width terrain . . . . . . . . . 98

3.30 Deformed contour for a $5000 \lambda$ terrain profile . . . . . . . . . . 101

3.31 Scattered field from a $5000 \lambda$ width terrain . . . . . . . . . . . 101

3.32 Deformed contour for a $10000 \lambda$ terrain profile . . . . . . . . 102

3.33 Scattered field from a $10000 \lambda$ width terrain . . . . . . . . . . 102

3.34 Deformed contour for a $20000 \lambda$ terrain profile . . . . . . . . . 103

3.35 Scattered field from a $20000 \lambda$ width terrain . . . . . . . . . . 103

4.1 ITU-R Rec.370 Curves. . . . . . . . . . . . . . . . . . . . . . . . 109

4.2 ITU-R Rec.529 Curves. . . . . . . . . . . . . . . . . . . . . 111

4.3 ITU-R Rec.1546 Curves. . . . . . . . . . . . . . . . . . . . . 113

$4.4 \quad$ FCC Curves. . . . . . . . . . . . . . . . . . . . . . . . . . . 114 
4.5 Path profile model for single knife edge diffraction.

4.6 Epstein-Peterson method geometry. . . . . . . . . . . . . 115

4.7 Bullington method geometry. . . . . . . . . . . 116

4.8 Deygout method geometry. . . . . . . . . . . . . . . 116

4.9 Downhill terrain profile and deformed path. . . . . . . . . 118

4.10 Uphill terrain profile and deformed path. . . . . . . . . . . . . 118

4.11 FreeSpace vs. IE over downhill terrain for $200 \mathrm{MHz}$. . . . . . . . 120

4.12 ITUR370 vs. IE over downhill terrain for $200 \mathrm{MHz}$. . . . . . . . 121

4.13 ITUR529 vs. IE over downhill terrain for $200 \mathrm{MHz} . . . . .122$

4.14 ITUR1546 vs. IE over downhill terrain for $200 \mathrm{MHz} . \quad$. . . . . . 123

4.15 FCC vs. IE over downhill terrain for $200 \mathrm{MHz} . . . . . . . .124$

4.16 FreeSpace vs. IE over uphill terrain for $200 \mathrm{MHz}$. . . . . . . . . . 125

4.17 ITUR370 vs. IE over uphill terrain for $200 \mathrm{MHz} . \ldots . . . .126$

4.18 ITUR529 vs. IE over uphill terrain for $200 \mathrm{MHz} . \ldots . . . .127$

4.19 ITUR1546 vs. IE over uphill terrain for $200 \mathrm{MHz}$. . . . . . . . . 128

4.20 FCC vs. IE over uphill terrain for $200 \mathrm{MHz} . \quad$. . . . . . . . . . . 129

4.21 Deformed path of downhill profile at $500 \mathrm{MHz}$. . . . . . . . . 132

4.22 Deformed path of uphill profile at $500 \mathrm{MHz}$. . . . . . . . . . . 132

4.23 FreeSpace vs. IE over downhill terrain for $500 \mathrm{MHz}$. . . . . . . . 134

4.24 ITUR370 vs. IE over downhill terrain for $500 \mathrm{MHz}$. . . . . . . . 135 
4.25 ITUR529 vs. IE over downhill terrain for $500 \mathrm{MHz} . . . .136$

4.26 ITUR1546 vs. IE over downhill terrain for $500 \mathrm{MHz} . \quad$. . . . . . . 137

4.27 FCC vs. IE over downhill terrain for $500 \mathrm{MHz} . \ldots . . . .138$

4.28 FreeSpace vs. IE over uphill terrain for $500 \mathrm{MHz}$. . . . . . . . . 139

4.29 ITUR370 vs. IE over uphill terrain for $500 \mathrm{MHz} . . . . . .140$

4.30 ITUR529 vs. IE over uphill terrain for $500 \mathrm{MHz} . . . . . .141$

4.31 ITUR1546 vs. IE over uphill terrain for $500 \mathrm{MHz} . . . . . .142$

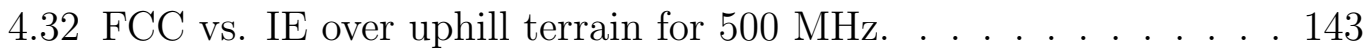

4.33 Deformed path of downhill profile at $890 \mathrm{MHz}$. . . . . . . . . . 145

4.34 Deformed path of uphill profile at $890 \mathrm{MHz}$. . . . . . . . . . . . 145

4.35 FreeSpace vs. IE over downhill terrain for $890 \mathrm{MHz}$. . . . . . . . 147

4.36 ITUR370 vs. IE over downhill terrain for $890 \mathrm{MHz} . \ldots . . .148$

4.37 ITUR529 vs. IE over downhill terrain for $890 \mathrm{MHz} . . . . .149$

4.38 ITUR1546 vs. IE over downhill terrain for $890 \mathrm{MHz}$. . . . . . . . 150

4.39 FCC vs. IE over downhill terrain for $890 \mathrm{MHz} . . . . . .151$

4.40 FreeSpace vs. IE over uphill terrain for $890 \mathrm{MHz}$. . . . . . . . . . 152

4.41 ITUR370 vs. IE over uphill terrain for $890 \mathrm{MHz} . \ldots . . .153$

4.42 ITUR529 vs. IE over uphill terrain for $890 \mathrm{MHz} . . . . . .154$

4.43 ITUR1546 vs. IE over uphill terrain for $890 \mathrm{MHz}$. . . . . . . . . 155

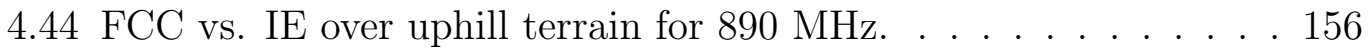


$4.45 \mathrm{MD}$ corrections vs. IE over downhill terrain for $200 \mathrm{MHz}$. . . . . 158

4.46 MD corrections vs. IE over downhill terrain for $500 \mathrm{MHz}$. . . . . 159

$4.47 \mathrm{MD}$ corrections vs. IE over downhill terrain for $890 \mathrm{MHz}$. . . . . 160

4.48 MD corrections vs. IE over uphill terrain for $200 \mathrm{MHz}$. . . . . . 161

$4.49 \mathrm{MD}$ corrections vs. IE over uphill terrain for $500 \mathrm{MHz}$. . . . . . . 162

4.50 MD corrections vs. IE over uphill terrain for $890 \mathrm{MHz}$. . . . . . 163 


\section{List of Tables}

2.1 Computational Cost . . . . . . . . . . . . . . . 36

2.2 Computational Cost . . . . . . . . . . . . . . . 50

3.1 Computational Cost . . . . . . . . . . . . . . 88

3.2 Study Parameters . . . . . . . . . . . . . . . . . . . . . 92

3.3 Study Parameters . . . . . . . . . . . . . . . . . . . . . 99

3.4 Computational Cost for the FBSA . . . . . . . . . . . . 104

4.1 Study Parameters for $200 \mathrm{MHz}$. . . . . . . . . . . . . 117

4.2 Absolute error values for $200 \mathrm{MHz}$. . . . . . . . . . . 130

4.3 Study Parameters for $500 \mathrm{MHz}$. . . . . . . . . . . . . . . . . . 131

4.4 Absolute error values for $500 \mathrm{MHz}$. . . . . . . . . . . . . 144

4.5 Study Parameters for $890 \mathrm{MHz}$. . . . . . . . . . . . . 146

4.6 Absolute error values for $890 \mathrm{MHz}$. . . . . . . . . . . . . . . 157

4.7 Absolute error values for downhill profile . . . . . . . . . . . 164

4.8 Absolute error values for uphill profile . . . . . . . . . . . 164 
4.9 Computational Cost for the FBSA . . . . . . . . . . . 165 
To My Nur and My Family ... 


\section{Chapter 1}

\section{INTRODUCTION}

The wireless communications era was born in 1970s with the development of highly reliable, miniature, solid-state radio frequency hardware. Since then, new wireless communications methods and services have been enthusiastically adopted by people throughout the world. The future growth of wireless communications systems will be tied more closely to radio spectrum allocations and regulatory decisions.

The frequency assignment problem has a significant role in sharing wellplanned frequency spectrum and obtaining the maximum serviceability. Frequency allocation and planning is a comprehensive study that implies coverage analysis, establishing locations of transmitters or receivers, computation of the interference over the candidate frequencies. Therefore, mobile radio planning requires the accurate computation of electromagnetic field strengths over large areas and in a wide variety of environments. In this regard, the problem is concerned with finding solutions and direct approaches to Maxwell's equations over randomly rough surfaces, such as integral equation based approaches. 


\subsection{One-Dimensional Rough Surface Scattering Problem}

Electromagnetic scattering from rough surfaces has been extensively treated in the literature. A recent review can be found in a special issue about this topic [1]. Most recent advances have been focused on the direct numerical simulation of the scattering problem. Numerical techniques based on integral equation formulations, such as the well-known Method of Moments (MoM) [2], are apparently some of the few sufficiently accurate and robust methods for low-grazing-angle scattering problems.

The primary factor limiting the use of the MoM in the calculation of electromagnetic scattering from rough surfaces is that a linear system of equations must be solved to yield the currents induced on the scatterer. Direct solution methods such as $L U$ decomposition are $O\left(N^{3}\right)$ operations, where $N$ is the number of unknowns in the discretized representation of the surface current. As the size of the surface increases, the computational expense of these operations becomes prohibitive. This has led to the development of iterative schemes that solve for the surface current in $O\left(N^{2}\right)$ steps.

The Forward-Backward Method (FBM) is a stationary iterative technique for solving linear equation systems resulting from electromagnetic rough surface scattering problems, which was developed for solving the magnetic field integral equation (MFIE) for perfect electrically conducting (PEC) surface by Holliday et al. [3], [4]. The method has been proposed for calculating the electromagnetic current on ocean-like PEC surfaces at low grazing angles. A similar approach was developed simultaneously by Kapp and Brown and called the Method of Ordered Multiple Interactions (MOMI) [5]. Both of them are based on splitting the current at each point into two components: the forward contribution due to the incident field and the radiation of the current elements located in front of 
the receiving element and the backward contribution due to the current elements located beyond the receiving element. The forward component is first found over the whole surface and then it is used to determine the backward contribution. This is repeated in an iterative process until a converged solution is reached. These methods have shown a very fast convergence, obtaining accurate results within very few iterations. However, the operational count is still $O\left(N^{2}\right)$, which prohibits the application of the FBM to very large-scale scattering problems.

The Spectral Acceleration algorithm was proposed to overcome the computational limitation of the FBM over one-dimensional slightly rough PEC surfaces by Chou and Johnson in [6], [7]. This algorithm accelerates the matrix-vector multiplications in the FBM and is based on a spectral representation of the twodimensional Green's function and an appropriate contour deformation. Consequently, the computational cost and memory requirements are reduced to $O(N)$, so that the Spectrally Accelerated Forward-Backward Method (FBSA) can be applied over very large one-dimensional surfaces. It should be noted that these original implementations of the spectral acceleration algorithm were developed to analyze quasi-planar (slightly rough) surfaces such as ocean-like surfaces, and becomes not suitable for very undulating geometries.

\subsection{Propagation Prediction Approaches}

Because of the computational limitations of integral equation based methods due to the large number of surface unknowns, the development of automatic tools for radio coverage prediction over geographical data is a growing interest area. Therefore, the coverage and propagation loss study for wireless communications has become a focus of interest and a great number of propagation models have been developed. 
According to their nature, the propagation models can be classified as empirical, semi-empirical (or semi-deterministic), and deterministic models.

Empirical models are described by equations or curves derived from statistical analysis of a large number of measured data. Among the empirical methods for predicting the field strength and path loss over terrain profiles for VHF-UHF frequencies, International Telecommunication Union Recommendations [8]-[10] and Federal Communications Commission curves [11] are considered to be the most significant ones. These models are simple and do not require detailed information about the environment. They are also easy and fast to apply because the estimation is usually obtained from experimental measurements. However, they can not provide a very accurate estimation of the scattered field or the path loss for an arbitrary environment.

Deterministic models are site-specific calculation methods which physically simulate the propagation of radio waves. Therefore the effect of the environment on the propagation parameters can be taken into account more accurately than in empirical models. Most of the deterministic models are based on ray-optical modelling approaches. The serious drawback of ray-optical methods is the computational complexity. Another kind of deterministic methods that has been studied extensively are those derived from the parabolic wave equation (PWE) approximation to the Helmholtz equation, in both integral and differential forms [12]-[14]. The PWE method is useful in problems where the energy is expected to propagate dominantly in a particular direction. The parabolic wave equation method allows handling the tropospheric refractive index variations, but they neglect the contribution of the backscattered field that is important in some cases and assume only forward propagation.

Semi-deterministic models result from an empirical modification of deterministic models in order to improve the agreement with measurement. These 
methods require more detailed information about the environment than the empirical methods but not as much as the deterministic models. Many of them are based on the high frequency asymptotic techniques such as spherical earth diffraction, multiple knife edge diffraction, geometrical optics and geometrical theory of diffraction. One such model, known as the Spherical Earth Knife Edge [15], uses a weighted average of analytic solutions for the multi-path, spherical earth, and knife-edge diffraction contributions which depends on the transmitter, receiver and terrain geometries. Another approach is the GTD model in [16] based on the application of the wedge diffraction modified to include finite conductivity and local roughness effects. Both methods have shown reasonable agreement with experimental data but there are significant differences in some cases that are difficult to explain. Besides, large number of knife edges or wedges required to model a terrain profile makes their application to real problems very cumbersome.

For the practical application of propagation models there is an important tradeoff between the accuracy of the prediction and the speed with which the prediction can be made. Inserting semi-deterministic reflection and multiple diffraction (RMD) corrections, into empirical prediction models yields more accurate results than the empirical results. Also, they are relatively easy and fast to apply. Therefore, empirical models with RMD corrections are commonly used to predict the field strengths over terrain profiles.

\subsection{Integral Equation Based Methods for Ter- rain Propagation}

Most of the radio propagation prediction methods are obtained by a combination of guesswork and analysis so that they can not give clear physical picture of the 
propagation process. So, choosing the best prediction model among a great number of methods becomes an important problem.

In this regard, numerical methods, such as integral equation (IE) based methods, become very desirable because they would avoid any kind of uncertainty in the electromagnetic analysis and hence, could be used to check the sensitivity of the true solution to the input terrain data. Besides, they could be used as a reference solution as an alternative to measurements to validate and clarify the limitations of other heuristic and intuitive methods involving approximations. Majority of the integral equation methods are based on the Method of Moments formulation [2]. The application of the MoM for the electrically large scattering surfaces implies the use of a very large number of surface unknowns $N$. Therefore, the solution of this kind of problems implies a very high computational cost in terms of $\mathrm{CPU}$ time and storage requirements. In this sense, the recently developed fast solvers for surface integral equation problems provide an alternative.

The first application of an integral equation method to the terrain propagation problem can be found in [17], where an IE is applied over small terrain profiles. Nevertheless, the application of this method to electrically large terrain profiles becomes impractical, due to the computational cost associated. Later on, in [18], a surface integral equation is derived and simplified with some assumptions such as neglecting back scattering and perfect magnetic conductivity, which make the method more efficient but still very time consuming and less accurate. In [19], an integral equation formulation is combined with an iterative version of the MoM, known as the banded matrix iterative approach (BMIA). As in the previous case, this method remains computationally complex, although a parallel implementation of the method allows the solution of some practical problems in the VHF band. A more efficient solution can be found in [20], where the fast far field approximation (FAFFA) was introduced and modified in an integral 
equation formulation for the terrain propagation problem. The FAFFA reduces the operational cost of the previous IE methods from $O\left(N^{2}\right)$ per iteration to $O\left(N^{4 / 3}\right)$. Finally, López et al. modified the spectral acceleration algorithm in order to implement FBSA to very undulating rough surfaces such as terrain profiles in [21] and the computational cost is reduced to $O(N)$.

This thesis aims to examine the most common propagation prediction models and multiple diffraction loss correction methods, and find out the most preferable ones in terms of accuracy. In order to achieve these goals, in this work, various implementations of conventional FBM and FBSA over various kinds of rough surface profiles are presented.

The conventional FBM is shown to be used as a reference solution instead of MoM, with its very accurate solutions and rapid convergence ability, for large numbers of surface unknowns where MoM fails because of the operational cost. Furthermore, it is applied over terrain profiles and used as a reference solution in order to examine the FBSA results. After several numerical experimentation over the FBSA, advantages and limitations of the method are detected, and FBSA is shown to be useful.

The main novelty of this work is to examine the propagation prediction models and multiple diffraction correction models against FBSA solutions. A great number of implementations of the propagation models are compared with the FBSA solutions over various terrain profiles for different frequency sets. According to the examinations, the most accurate propagation models and diffraction correction methods are proposed.

All fields and currents in this work are considered to have a time-harmonic dependence of the form $e^{j w t}$, that is suppressed from the expressions. The angular frequency is $\omega$ and $k$ is the wave number of the medium, which is assumed to be free space, above the rough surface. 


\section{Chapter 2}

\section{FORWARD-BACKWARD}

\section{METHOD}

The Forward-Backward Method (FBM) is a stationary iterative technique for solving linear system of equations resulting from electromagnetic rough surface scattering problems, which was developed for solving the magnetic field integral equation (MFIE) for perfect electrically conducting (PEC) surface by Holliday et al. [3], [4]. The method has been proposed for calculating the electromagnetic current on ocean-like PEC surfaces at low grazing angles. A similar approach was developed simultaneously by Kapp and Brown and called the Method of Ordered Multiple Interactions (MOMI) [5]. Both methods provide accurate results within very few iterations, causing a computational cost and a memory requirement of $O\left(N^{2}\right)$, where $N$ is the number of surface unknowns.

Since FBM and MOMI present very fast convergence, a great number of studies have been implemented in recent years. Holliday et al. extended FBM to imperfect conductors in [22]. In [23], a curvature term was included in the propagator matrix for MOMI in order to eliminate the undesired sampling sensitivity effect. Chou and Johnson combined FBM with electric field integral equation 
(EFIE).Furthermore they proposed an acceleration algorithm based on the spectral representation of Green's function which reduces the operational count to $O(N)$ for PEC surfaces [6], [7]. A combined field approach for scattering from infinite elliptical cylinders using MOMI was presented in [24].

West and Sturm investigated convergence performances and limitations of both methods by comparing them with several stationary and non-stationary iterative approaches [25]. The FBM may not exhibit convergent behavior for surfaces where the Method of Moment (MoM) current elements are not numbered sequentially as a function of increasing $x$ coordinate such as a ship or a large breaking wave on the ocean surface. The Generalized Forward-Backward Method (GFBM), which is a hybrid method based on a combination of the conventional FBM with MoM, was proposed to overcome this limitation in [26].

Chou and Johnson extended the Spectrally Accelerated Forward-Backward Method (FBSA) formulation to treat impedance surfaces [27]. Wang et al. applied FBM to high frequency radio wave propagation problems over forest canopies [28]. Chou presented applications of FBM and GFBM in the analysis of large array problems in [29], [30]. A multilevel version of the spectral acceleration algorithm was introduced in [31]. López et al. modified the spectral acceleration algorithm in order to implement FBSA to very undulating rough surfaces such as terrain profiles [21]. The spectral acceleration algorithm was adapted to the GFBM in [32]. Çivi presented an extension of FBM with discrete Fourier transform based acceleration algorithm for the efficient analysis of large printed dipole arrays [33].

This chapter is devoted to the discussion of the Forward-Backward Method. Corresponding integral and matrix equations for horizontal and vertical polarizations, and FBM formulations are described in Sections 2.1 and 2.2, respectively. Section 2.3 presents numerical results and limitations of the FBM over several sample rough surfaces. 


\subsection{Integral and Matrix Equations for the Forward-Backward Method}

In this chapter, our purpose is to compute the scattered field over a onedimensional rough surface profile which is illuminated by an electromagnetic source. Figure 2.1 illustrates such a rough surface that is characterized with the curve $C$ defined by $z=f(x)$, along the $x$-axis. This surface is illuminated by an incident field $\left\{\mathbf{E}^{i n c}(\boldsymbol{\rho}), \mathbf{H}^{i n c}(\boldsymbol{\rho})\right\}$, where $\boldsymbol{\rho}=\hat{x} x+\hat{z} z$ is the two-dimensional position vector denoting the position along the surface. The terrain is considered to be an imperfect conductor $\left(\epsilon_{r}(\boldsymbol{\rho}), \mu_{r}(\boldsymbol{\rho})\right)$.

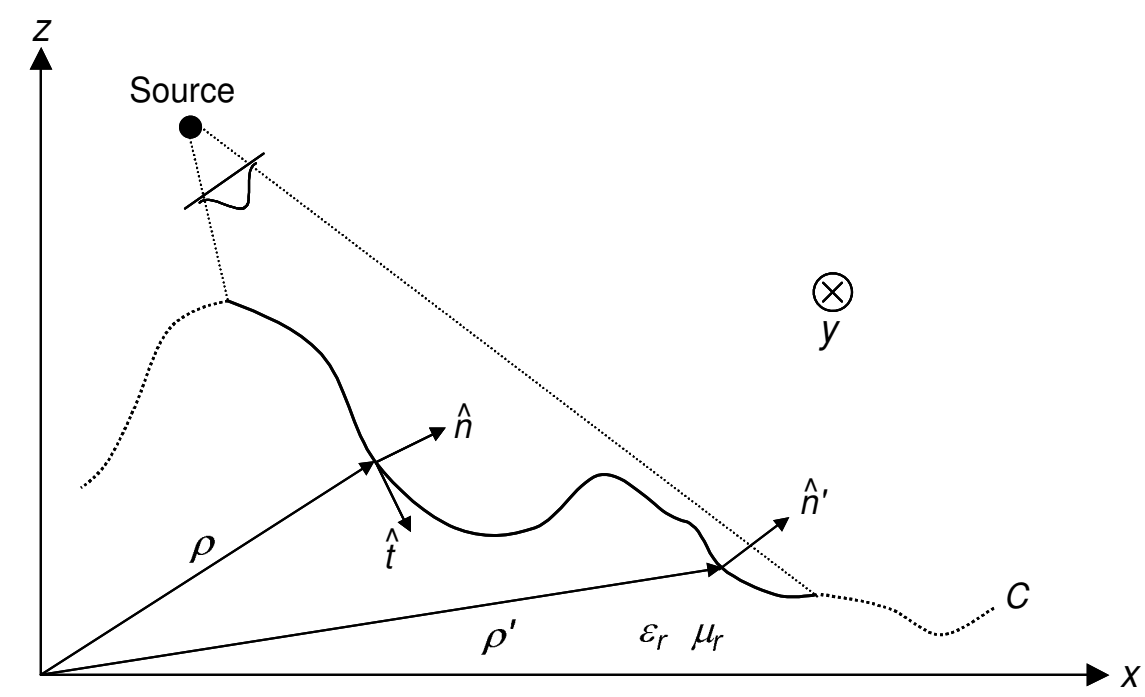

Figure 2.1: Problem Geometry

Assuming the relative permittivity of the scattering surface is large, an approximate the impedance boundary condition (IBC) can be used. If an IBC is valid, the surface may be treated using a single surface integral equation. Detailed information about impedance boundary condition can be found in [34]-[37].

An equivalent exterior problem for the rough surface profile illustrated in Figure 2.1 can be obtained using electric and magnetic sources $\mathbf{J}$ and $\mathbf{K}$, respectively, 
defined on the surface according to

$$
\begin{array}{r}
\mathbf{J}=\hat{n} \times \mathbf{H} \\
\mathbf{K}=\mathbf{E} \times \hat{n},
\end{array}
$$

and radiating in an infinite space with the same parameters as the exterior medium. Since the relative permittivity is large, the equivalent sources of $(2.1)$ and (2.2) can satisfy the IBC [35]

$$
\mathbf{K}(\boldsymbol{\rho})=\eta_{s}(\boldsymbol{\rho}) \mathbf{J}(\boldsymbol{\rho}) \times \hat{n}(\boldsymbol{\rho})
$$

where $\hat{n}$ is the unit normal vector to the surface and $\eta_{s}$ is the surface impedance which may vary along the surface. Integral equations for the problem can be formulated to relate the incident electric or magnetic fields to the equivalent sources.

In order to examine the scattering problem for a general wave polarization, it is most convenient to decompose the electric field into its perpendicular and parallel components relative to the plane of incidence, and analyze each one of them individually. The total field will be the vector sum of these two polarizations.

The transverse magnetic (TM) case, in which the electric field is perpendicular to the plane of incidence, is defined as the horizontal polarization case; while the transverse electric (TE) case, in which the electric field is parallel to the plane of incidence, is called as the vertical polarization case. Both horizontal and vertical polarization cases are examined in the following subsections.

\subsubsection{EFIE Formulation for Horizontal Polarized Inci- dence on Non-PEC Surfaces}

To compute the scattered field, unknown current induced on the surface has to be found for a given incident field, which may be radiated by any kind of source. If 
the incident field on the scattering surface in Figure 2.1 is horizontally polarized $\left(\mathbf{E}^{i n c}=\hat{y} E^{i n c}\right)$, and if an impedance boundary condition is valid, then equivalent sources have components $J_{y}$ and $K_{t}$, and the IBC reduces to

$$
K_{t}(\boldsymbol{\rho})=E_{y}(\boldsymbol{\rho})=\eta_{s}(\boldsymbol{\rho}) H_{t}(\boldsymbol{\rho})=\eta_{s}(\boldsymbol{\rho}) J_{y}(\boldsymbol{\rho})
$$

$\hat{t}=\hat{y} \times \hat{n}$ is the unit tangent vector along the surface, and hence, $K_{t}$ denotes the tangential component of the equivalent magnetic source. Then an electric field integral equation given by

$$
-\mathbf{E}^{i n c}(\boldsymbol{\rho})=-\eta_{s}(\boldsymbol{\rho}) J_{y}(\boldsymbol{\rho})+\mathbf{E}^{s c a t}(\boldsymbol{\rho})
$$

is valid on the scattering surface.

The electric field integral equation (EFIE) can be written entirely in terms of the equivalent electric current density $J_{y}$ on the surface as

$$
-E_{y}^{i n c}(\boldsymbol{\rho})=-\eta_{s}(\boldsymbol{\rho}) J_{y}(\boldsymbol{\rho})-j \omega A_{y}-\frac{1}{\epsilon}\left\{\frac{\partial F_{x}}{\partial z}-\frac{\partial F_{z}}{\partial x}\right\}
$$

where $\mathbf{A}$ and $\mathbf{F}$ are the magnetic and electric vector potentials, respectively, and can be expressed as

$$
\begin{gathered}
A_{y}(\boldsymbol{\rho})=\mu \int_{C} J_{y}\left(\boldsymbol{\rho}^{\prime}\right) G\left(\boldsymbol{\rho}, \boldsymbol{\rho}^{\prime}\right) d \rho^{\prime} \\
\mathbf{F}_{t}(\boldsymbol{\rho})=\epsilon \int_{C} \hat{t}\left(\boldsymbol{\rho}^{\prime}\right) \eta_{s}\left(\boldsymbol{\rho}^{\prime}\right) J_{y}\left(\boldsymbol{\rho}^{\prime}\right) G\left(\boldsymbol{\rho}, \boldsymbol{\rho}^{\prime}\right) d \rho^{\prime}
\end{gathered}
$$

where $\mu$ and $\epsilon$ are the permeability and permittivity of the medium above the rough surface, respectively. $\mathbf{F}_{t}$ denotes the tangential component of the electric vector potential. $G$ is the two-dimensional Green's function expressed as,

$$
G\left(\boldsymbol{\rho}, \boldsymbol{\rho}^{\prime}\right)=\frac{1}{4 j} H_{0}^{(2)}(k R)
$$

where $H_{0}^{(2)}$ is the second-kind Hankel function with order zero and

$$
R=\sqrt{\left[x(\boldsymbol{\rho})-x\left(\boldsymbol{\rho}^{\prime}\right)\right]^{2}+\left[z(\boldsymbol{\rho})-z\left(\boldsymbol{\rho}^{\prime}\right)\right]^{2}} .
$$

Here primed coordinates denote the source locations, while unprimed coordinates represent observation points on the surface. 
Substituting (2.7) and (2.8) into (2.6), the electric field integral equation can be rewritten as

$$
\begin{aligned}
-E_{y}^{i n c}(\boldsymbol{\rho}) & =-\eta_{s}(\boldsymbol{\rho}) J_{y}(\boldsymbol{\rho})-j \omega \mu \int_{C} J_{y}\left(\boldsymbol{\rho}^{\prime}\right) G\left(\boldsymbol{\rho}, \boldsymbol{\rho}^{\prime}\right) d \rho^{\prime} \\
& +\int_{C} \eta_{s}\left(\boldsymbol{\rho}^{\prime}\right) J_{y}\left(\boldsymbol{\rho}^{\prime}\right) \frac{\partial}{\partial n^{\prime}} G\left(\boldsymbol{\rho}, \boldsymbol{\rho}^{\prime}\right) d \rho^{\prime}
\end{aligned}
$$

where, $J_{y}$ is the surface electrical current on $C$ and $\frac{\partial}{\partial n^{\prime}} G$ is the derivative of the two-dimensional Green's function with respect to $\hat{n}^{\prime}$, the normal vector to the surface at the source point $\boldsymbol{\rho}^{\prime}$.

Assuming that the incident field is finite, the surface and the integration in (2.11) can be confined to a finite region, though the profile $C$ is arbitrarily extended to infinity. Therefore, (2.11) can be solved using a Method of Moments (MoM) discretization process [2].

\section{The Method of Moments Solution}

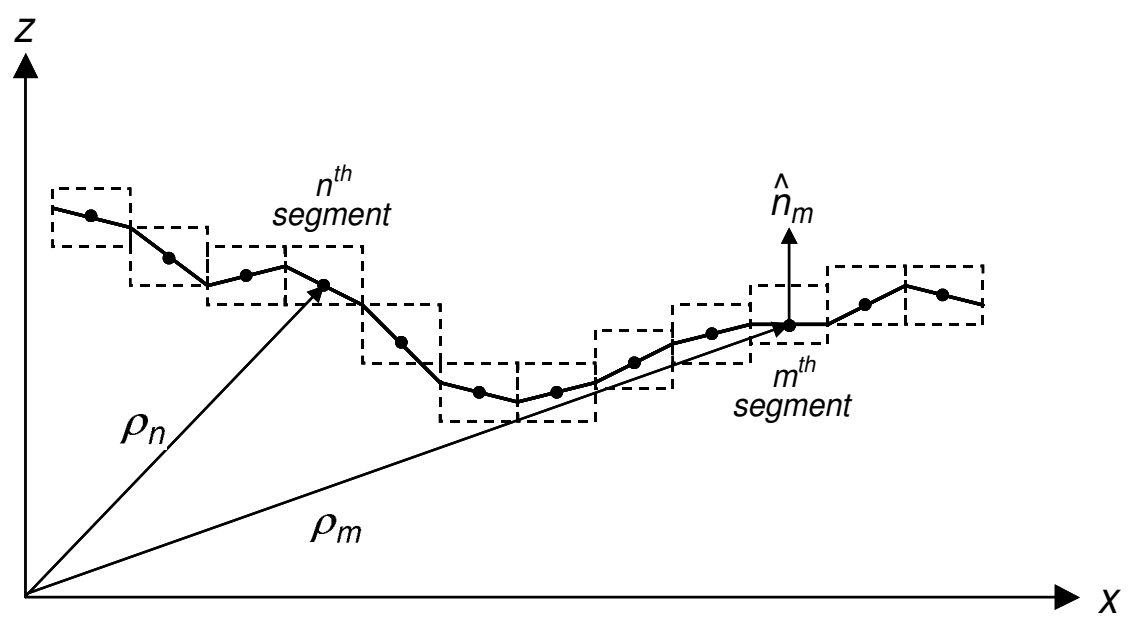

Figure 2.2: Surface Discretization

It is necessary to solve (2.11) for the unknown $J_{y}\left(\boldsymbol{\rho}^{\prime}\right)$ and that is an operator inversion problem. (2.11) is an integral equation that can be used to find the unknown induced current $J_{y}\left(\boldsymbol{\rho}^{\prime}\right)$ based on the incident electric field $-E_{y}^{\text {inc }}(\boldsymbol{\rho})$. The solution may be reached numerically by reducing (2.11) to a series of linear 
algebraic equations that may be solved by conventional matrix equations techniques. To facilitate this, the unknown current density $J_{y}\left(\boldsymbol{\rho}^{\prime}\right)$ is approximated by an expansion of $N$ known terms with constant, but unknown coefficients:

$$
J_{y}\left(\boldsymbol{\rho}^{\prime}\right) \cong \sum_{m=1}^{N} I_{m} p_{m}\left(\boldsymbol{\rho}^{\prime}\right) .
$$

The surface is now divided into $N$ segments as illustrated in Figure 2.2. The $p_{m}\left(\boldsymbol{\rho}^{\prime}\right)$ functions in the expansion (2.12) are chosen for their ability to accurately model the unknown quantity, while minimizing computation. They are often referred to as basis or expansion functions. To avoid the computational cost, subdomain piecewise constant or pulse functions will be used. These functions are defined to be of a constant value over one segment and zero elsewhere, such that

$$
p_{m}\left(\boldsymbol{\rho}^{\prime}\right)= \begin{cases}1 & , \text { if } \boldsymbol{\rho}^{\prime} \in \text { segment } m \\ 0 & , \text { otherwise }\end{cases}
$$

Substituting (2.12) into (2.11) and evaluating (2.11) at a fixed observation point on the surface such as $\boldsymbol{\rho}_{n}$, produces an integrand that is solely a function of $\boldsymbol{\rho}^{\prime}$. Obviously this leads to one equation with $N$ unknowns $I_{m}$. In order to obtain a solution for these $N$ amplitude constants, $N$ linearly independent equations are necessary. These equations may be produced by choosing an observation point $\boldsymbol{\rho}_{n}$ on the surface at the center of each segment as shown in Figure $2.2(n=$ $1,2, \ldots, N)$. This will result in one equation corresponding to each observation point. Since the integral in (2.11) is nonsingular, interchanging the integration and summation,

$$
\begin{aligned}
-E_{y}^{i n c}\left(\boldsymbol{\rho}_{n}\right) \cong & -\eta_{s}\left(\boldsymbol{\rho}_{n}\right) I_{n}-j \omega \mu \sum_{m=1}^{N} I_{m} \int_{\Delta x_{m}} G\left(\boldsymbol{\rho}_{n}, \boldsymbol{\rho}_{m}\right) d \rho^{\prime} \\
& +\sum_{m=1}^{N} I_{m} \int_{\Delta x_{m}} \eta_{s}\left(\boldsymbol{\rho}_{m}\right) \frac{\partial}{\partial n_{m}} G\left(\boldsymbol{\rho}_{n}, \boldsymbol{\rho}_{m}\right) d \rho^{\prime}
\end{aligned}
$$

are valid for $N$ such points of observation. The $N \times N$ system produced by (2.14) can be written more concisely using matrix notation as 


$$
-\left[\begin{array}{c}
E_{y}^{i n c}\left(\boldsymbol{\rho}_{1}\right) \\
E_{y}^{i n c}\left(\boldsymbol{\rho}_{2}\right) \\
\vdots \\
E_{y}^{i n c}\left(\boldsymbol{\rho}_{N}\right)
\end{array}\right]=\left[\begin{array}{cccc}
Z_{11} & Z_{12} & \ldots & Z_{1 N} \\
Z_{21} & Z_{22} & \ldots & Z_{2 N} \\
\vdots & \vdots & \ldots & \vdots \\
Z_{N 1} & Z_{N 2} & \ldots & Z_{N N}
\end{array}\right]\left[\begin{array}{c}
I_{1} \\
I_{2} \\
\vdots \\
I_{N}
\end{array}\right]
$$

or

$$
\left[V_{n}\right]=\left[Z_{n m}\right] \cdot\left[I_{m}\right]
$$

In summary, the solution of (2.11) for the current distribution on a rough surface has been accomplished by approximating the unknown with pulse basis functions, dividing the surface into segments, and then sequentially enforcing (2.11) at the center of each segment to form a set of linear equations. The procedure that is followed to convert the continuous integral equation to a discrete matrix equation is a special case of a general approach known as Method of Moments. In this special case the basis functions are pulse functions and weighting (testing) functions are impulses. This is also called the point matching with pulse basis functions [2].

The entries of the $N \times N$ matrix in (2.15) represent the self and mutual impedances between different segments in the model, thus, this matrix is called as the moment method impedance matrix. The entries of the impedance matrix in (2.15) are given by,

$$
Z_{n m}=\int_{\Delta x_{m}}\left[-j \omega \mu G\left(\boldsymbol{\rho}_{n}, \boldsymbol{\rho}_{m}\right)+\eta_{m} \frac{\partial}{\partial n_{m}} G\left(\boldsymbol{\rho}_{n}, \boldsymbol{\rho}_{m}\right)\right] d \rho^{\prime}
$$

where $\boldsymbol{\rho}_{n}$ denotes the observation point which is considered to be located on the center of the $n$th segment, while $\boldsymbol{\rho}_{m}$ represents the source point on the center of the $m$ th. If the segments are small compared to the wavelength, typically $\frac{\lambda}{10}$, the elements of the impedance matrix may be approximated as,

$$
\begin{aligned}
Z_{n m} & \cong-\frac{\omega \mu}{4} \Delta x_{m} H_{0}^{(2)}\left(k\left|\boldsymbol{\rho}_{n}-\boldsymbol{\rho}_{m}\right|\right) \\
& -j \frac{k \eta_{m}}{4} \Delta x_{m} H_{1}^{(2)}\left(k\left|\boldsymbol{\rho}_{n}-\boldsymbol{\rho}_{m}\right|\right) \hat{n}_{m} \cdot \hat{\rho}_{n m}
\end{aligned}
$$


where $H_{1}^{(2)}$ is the second-kind Hankel function with order one, coming from the partial derivative of the Green's function and $\Delta x_{m}$ is the length of the $m$ th segment. Also, $\hat{\rho}_{n m}$ denotes a unit vector in the direction from source $\boldsymbol{\rho}_{m}$ to the receiving element $\boldsymbol{\rho}_{n}$, and $\hat{n}_{m}$ represents the unit normal vector of the surface at $\boldsymbol{\rho}_{m}$

Since the Hankel function is singular for $\boldsymbol{\rho}_{n}=\boldsymbol{\rho}_{m}$, the diagonal elements of the impedance matrix cannot be evaluated using (2.18). Moreover, accurate evaluation of the diagonal terms is very important, since they give a greater contribution to the solution of the system because of their relatively larger amplitudes. Therefore, the impedance matrix is diagonally dominant, and using the small argument series expansion of the Hankel functions, diagonal entries of the impedance matrix can be obtained as [38]

$$
Z_{m m} \cong-\frac{\omega \mu}{4} \Delta x_{m}\left[1-j \frac{2}{\pi} \ln \left(\frac{\gamma k \Delta x_{m}}{4 e}\right)\right]-\frac{\eta_{m}}{2}
$$

where $\gamma$ is the Euler constant 1.781072418 and $e=2.718281828$.

Note that, the expressions for the PEC case can be deduced by a simple manner through replacing $\eta_{m}$ by 0 . For the sake of completeness, the expressions for the PEC case are rewritten as follows:

$$
\begin{gathered}
Z_{n m} \cong-\frac{\omega \mu}{4} \Delta x_{m} H_{0}^{(2)}\left(k\left|\boldsymbol{\rho}_{n}-\boldsymbol{\rho}_{m}\right|\right) \\
Z_{m m} \cong-\frac{\omega \mu}{4} \Delta x_{m}\left[1-j \frac{2}{\pi} \ln \left(\frac{\gamma k \Delta x_{m}}{4 e}\right)\right] .
\end{gathered}
$$

(2.11) is now said to be discretized to form the matrix equation (2.15). The elements of the impedance matrix are obtained in (2.18) and (2.19) for mutual and self coupling terms, respectively. The system $\mathbf{V}=\overline{\mathbf{Z}} \cdot \mathbf{I}$ should be solved for unknown current coefficients, $\mathbf{I}=\left\{I_{m}\right\}$. 


\subsubsection{MFIE Formulation for Vertical Polarized Incidence on Non-PEC Surfaces}

If the incident field on the scattering surface in Figure 2.1 has a vertical polarization $\left(\mathbf{H}^{i n c}=\hat{y} H^{i n c}\right)$, and if an impedance boundary condition is valid along the surface, then equivalent sources have components $K_{y}$ and $J_{t}$, and the IBC reduces to

$$
K_{y}(\boldsymbol{\rho})=-E_{t}(\boldsymbol{\rho})=\eta_{s}(\boldsymbol{\rho}) H_{y}(\boldsymbol{\rho})=-\eta_{s}(\boldsymbol{\rho}) J_{t}(\boldsymbol{\rho})
$$

Although the MFIE formulation is generally used for closed surfaces; since the surface is assumed to be arbitrarily extended to infinity, a magnetic field integral equation can be used to model the vertical polarization problem. Thus, the magnetic field integral equation

$$
-H_{y}^{i n c}(\boldsymbol{\rho})=J_{t}(\boldsymbol{\rho})+H_{y}^{s c a t}(\boldsymbol{\rho})
$$

is valid on the scattering surface.

In terms of the tangential induced current $J_{t}$, the magnetic field integral equation can be expressed on the surface as

$$
-H_{y}^{i n c}(\boldsymbol{\rho})=J_{t}(\boldsymbol{\rho})-j \omega F_{y}-\frac{1}{\mu}\left\{\frac{\partial A_{z}}{\partial x}-\frac{\partial A_{x}}{\partial z}\right\}
$$

where

$$
\begin{gathered}
\mathbf{A}_{t}(\boldsymbol{\rho})=\mu \int_{C} \hat{t}\left(\boldsymbol{\rho}^{\prime}\right) J_{t}\left(\boldsymbol{\rho}^{\prime}\right) G\left(\boldsymbol{\rho}, \boldsymbol{\rho}^{\prime}\right) d \rho^{\prime} \\
F_{y}(\boldsymbol{\rho})=-\epsilon \int_{C} \eta_{s}\left(\boldsymbol{\rho}^{\prime}\right) J_{t}\left(\boldsymbol{\rho}^{\prime}\right) G\left(\boldsymbol{\rho}, \boldsymbol{\rho}^{\prime}\right) d \rho^{\prime}
\end{gathered}
$$

and $\hat{t}$ is the unit tangent vector along the surface.

Substituting (2.25) and (2.26) into (2.24), the magnetic field integral equation can be rewritten as

$$
\begin{aligned}
-H_{y}^{i n c}(\boldsymbol{\rho}) & =J_{t}(\boldsymbol{\rho})+j \omega \epsilon \int_{C} \eta_{s}\left(\boldsymbol{\rho}^{\prime}\right) J_{t}\left(\boldsymbol{\rho}^{\prime}\right) G\left(\boldsymbol{\rho}, \boldsymbol{\rho}^{\prime}\right) d \rho^{\prime} \\
& -\int_{C} J_{t}\left(\boldsymbol{\rho}^{\prime}\right) \frac{\partial}{\partial n^{\prime}} G\left(\boldsymbol{\rho}, \boldsymbol{\rho}^{\prime}\right) d \rho^{\prime}
\end{aligned}
$$


where, $G$ is the two-dimensional Green's function, given by (2.9), and $\frac{\partial}{\partial n^{\prime}} G$ is its derivative respect to $\hat{n}^{\prime}$, the normal vector to the surface at the source point $\boldsymbol{\rho}^{\prime}$.

Assuming that the incident field is finite, the surface profile $C$, which is arbitrarily extended to infinity, and the integration in (2.27) can be confined to a finite region. Therefore, applying the same discretization process illustrated in Figure 2.2, the equivalent current density can be approximated.

\section{The Method of Moments Solution}

(2.27) is an integral equation that can be used to find the unknown induced current $J_{t}\left(\boldsymbol{\rho}^{\prime}\right)$ based on the incident magnetic field $-H_{y}^{i n c}(\boldsymbol{\rho})$. The solution may be reached numerically by reducing (2.27) to a series of linear algebraic equations and then applying conventional matrix equation techniques. To facilitate this, the unknown current density $J_{t}\left(\boldsymbol{\rho}^{\prime}\right)$ is approximated by an expansion of $N$ known terms with constant, but unknown coefficients:

$$
J_{t}\left(\boldsymbol{\rho}^{\prime}\right) \cong \sum_{m=1}^{N} I_{m} p_{m}\left(\boldsymbol{\rho}^{\prime}\right)
$$

The surface is now divided into $N$ segments as illustrated in Figure 2.2. The $p_{m}\left(\boldsymbol{\rho}^{\prime}\right)$ functions in the expansion (2.28) are chosen to be subdomain piecewise constant or pulse functions in order to avoid the computational cost. These functions are defined to be of a constant value over one segment and zero elsewhere, such that

$$
p_{m}\left(\boldsymbol{\rho}^{\prime}\right)= \begin{cases}1 & , \text { if } \boldsymbol{\rho}^{\prime} \in \text { segment } m \\ 0 & , \text { otherwise }\end{cases}
$$

Note that, as $N \rightarrow \infty$, the approximated expression for the unknown current density approaches to the exact solution.

Substituting (2.28) into (2.27) and evaluating (2.27) at a fixed observation point on the surface such as $\boldsymbol{\rho}_{n}$, produces an integrand that is solely a function of $\boldsymbol{\rho}^{\prime}$. Obviously this yields one equation with $N$ unknowns $I_{m}$. In order to obtain a solution for these $N$ amplitude constants, $N$ linearly independent equations are 
necessary. These equations may be produced by choosing an observation point $\boldsymbol{\rho}_{n}$ on the surface at the center of each segment as shown in Figure $2.2(n=$ $1,2, \ldots, N)$. This will result in one equation corresponding to each observation point. Thus,

$$
\begin{aligned}
-H_{y}^{i n c}\left(\boldsymbol{\rho}_{n}\right) & \cong I_{n}+j \omega \epsilon \sum_{m=1}^{N} I_{m} \int_{\Delta x_{m}} \eta_{s}\left(\boldsymbol{\rho}_{m}\right) G\left(\boldsymbol{\rho}_{n}, \boldsymbol{\rho}_{m}\right) d \rho^{\prime} \\
& -\sum_{m=1}^{N} I_{m} \int_{\Delta x_{m}} \frac{\partial}{\partial n_{m}} G\left(\boldsymbol{\rho}_{n}, \boldsymbol{\rho}_{m}\right) d \rho^{\prime}
\end{aligned}
$$

are valid for $N$ such points of observation. The $N \times N$ system produced by (2.30) can be written more concisely using matrix notation as

$$
-\left[\begin{array}{c}
H_{y}^{i n c}\left(\boldsymbol{\rho}_{1}\right) \\
H_{y}^{i n c}\left(\boldsymbol{\rho}_{2}\right) \\
\vdots \\
H_{y}^{i n c}\left(\boldsymbol{\rho}_{N}\right)
\end{array}\right]=\left[\begin{array}{cccc}
Z_{11} & Z_{12} & \ldots & Z_{1 N} \\
Z_{21} & Z_{22} & \ldots & Z_{2 N} \\
\vdots & \vdots & \ldots & \vdots \\
Z_{N 1} & Z_{N 2} & \ldots & Z_{N N}
\end{array}\right]\left[\begin{array}{c}
I_{1} \\
I_{2} \\
\vdots \\
I_{N}
\end{array}\right]
$$

or

$$
\left[V_{n}\right]=\left[Z_{n m}\right] \cdot\left[I_{m}\right]
$$

In summary, the solution of (2.27) for the current distribution on a rough surface has been accomplished by approximating the unknown with pulse basis functions, dividing the surface into segments, and then sequentially enforcing (2.27) at the center of each segment (point matching) to form a set of linear equations [2]. The entries of the MoM impedance matrix in (2.31) are given by,

$$
Z_{n m}=\int_{\Delta x_{m}}\left[j \omega \epsilon \eta_{m} G\left(\boldsymbol{\rho}_{n}, \boldsymbol{\rho}_{m}\right)-\frac{\partial}{\partial n_{m}} G\left(\boldsymbol{\rho}_{n}, \boldsymbol{\rho}_{m}\right)\right] d \rho^{\prime}
$$

where $\boldsymbol{\rho}_{n}$ denotes the observation point which is considered to be located on the center of the $n$th segment, while $\boldsymbol{\rho}_{m}$ represents the source point on the center of the $m$ th. If the segments are small compared to the wavelength, typically $\frac{\lambda}{10}$, the elements of the impedance matrix may be approximated as,

$$
\begin{aligned}
Z_{n m} & \cong \frac{\omega \epsilon \eta_{m}}{4} \Delta x_{m} H_{0}^{(2)}\left(k\left|\boldsymbol{\rho}_{n}-\boldsymbol{\rho}_{m}\right|\right) \\
& +j \frac{k}{4} \Delta x_{m} H_{1}^{(2)}\left(k\left|\boldsymbol{\rho}_{n}-\boldsymbol{\rho}_{m}\right|\right) \hat{n}_{m} \cdot \hat{\rho}_{n m}
\end{aligned}
$$


where $H_{1}^{(2)}$ is the second-kind Hankel function with order one, coming from the partial derivative of the Green's function, and $\Delta x_{m}$ is the length of $m$ th segment. Also, $\hat{\rho}_{n m}$ denotes a unit vector in the direction from source $\boldsymbol{\rho}_{m}$ to the receiving element $\boldsymbol{\rho}_{n}$, and $\hat{n}_{m}$ represents the unit normal vector of the surface at $\boldsymbol{\rho}_{m}$.

Since the Hankel function is singular for $\boldsymbol{\rho}_{n}=\boldsymbol{\rho}_{m}$, the diagonal elements of the impedance matrix can be evaluated using the small argument series expansion of the Hankel functions. Thus, diagonal entries of the impedance matrix are obtained as [38]

$$
Z_{m m} \cong \frac{1}{2}+\frac{\omega \epsilon \eta_{m}}{4} \Delta x_{m}\left[1-j \frac{2}{\pi} \ln \left(\frac{\gamma k \Delta x_{m}}{4 e}\right)\right]
$$

where $\gamma$ is the Euler number 1.781072418 and $e=2.718281828$.

It should be noted that, the expressions for the PEC case can be obtained by a simple manner through replacing $\eta_{m}$ by 0 as:

$$
\begin{gathered}
Z_{n m} \cong j \frac{k}{4} \Delta x_{m} H_{1}^{(2)}\left(k\left|\boldsymbol{\rho}_{n}-\boldsymbol{\rho}_{m}\right|\right) \hat{n}_{m} \cdot \hat{\rho}_{n m} \\
Z_{m m} \cong \frac{1}{2}
\end{gathered}
$$

The MoM procedure generates an impedance matrix that has $N^{2}$ entries for $N$ surface unknowns. Each element of the matrix is calculated separately. For this reason, the processing time and memory requirement appears to be $O\left(N^{2}\right)$ to form the impedance matrix. Once the impedance matrix $\overline{\mathbf{Z}}$ is formed, the system $\mathbf{V}=\overline{\mathbf{Z}} \cdot \mathbf{I}$ should be solved for unknown current coefficients, $\mathbf{I}=\left\{I_{m}\right\}$. The direct solution methods such as Gaussian elimination or LU decomposition requires an $O\left(N^{3}\right)$ floating point operations. Therefore, processing time for the solution becomes $O\left(N^{3}\right)$ for direct solution methods. As the problem size becomes electrically larger, computational requirements of the MoM increases very rapidly. Therefore, instead of MoM, iterative techniques such as FBM, whose formulation is given in Section 2.2, can be used to reduce the operation count to $O\left(N^{2}\right)$. 


\subsection{The Formulation of the Forward-Backward Method}

Applying the discretization process, the integral equations modelling the original scattering problem are converted into matrix equations for both horizontal and vertical polarizations as,

$$
\mathbf{V}=\overline{\mathbf{Z}} \cdot \mathbf{I}
$$

where $\overline{\mathbf{Z}}$ is the MoM impedance matrix whose entries are given in (2.18) and (2.34) and column vector $\mathbf{V}$ elements are given by minus the incident field at matching points. The system defined by (2.38) should be solved for unknown current coefficients $\mathbf{I}=\left\{I_{m}\right\}$ in order to find the induced current on the surface. Instead of direct solution that causes $O\left(N^{3}\right)$ computational requirement, the Forward-Backward Method can be used for solving the matrix equation obtained for the EFIE, the MFIE or any combination of them.

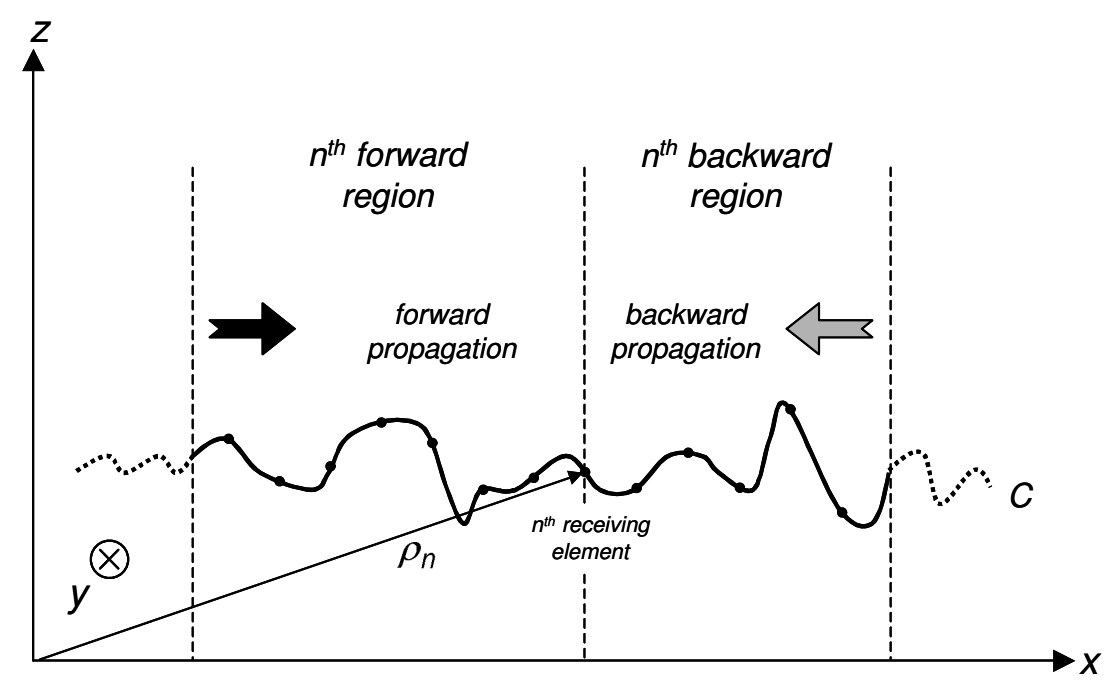

Figure 2.3: Forward and backward regions for the $n$th matching point

The Forward-Backward Method proposes a forward and backward decomposition over the matrices and vectors involved in (2.38) [7] 


$$
\begin{gathered}
\mathbf{I}=\mathbf{I}^{f}+\mathbf{I}^{b} \\
\overline{\mathbf{Z}}=\overline{\mathbf{Z}}^{f}+\overline{\mathbf{Z}}^{s}+\overline{\mathbf{Z}}^{b}
\end{gathered}
$$

where $\mathbf{I}^{f}$ is the forward component denoting the current distribution due to the wave propagation in the forward direction and $\mathbf{I}^{b}$ is the backward component representing the current distribution due to the wave propagation in the backward direction. In (2.40), $\overline{\mathbf{Z}}^{f}$ and $\overline{\mathbf{Z}}^{b}$ are the impedance matrices consisting of elements in the lower and upper triangular parts of $\overline{\mathbf{Z}}$ excluding the diagonal terms, respectively. It is noted that $\overline{\mathbf{Z}}^{s}$ is a diagonal matrix consisting only of the self impedances of all surface segments.

Using (2.39) and (2.40), the matrix equation given by (2.38) can be separated into two matrix equations:

$$
\begin{gathered}
\overline{\mathbf{Z}}^{s} \cdot \mathbf{I}^{f}=\mathbf{V}-\overline{\mathbf{Z}}^{f} \cdot\left(\mathbf{I}^{f}+\mathbf{I}^{b}\right) \\
\overline{\mathbf{Z}}^{s} \cdot \mathbf{I}^{b}=-\overline{\mathbf{Z}}^{b} \cdot\left(\mathbf{I}^{f}+\mathbf{I}^{b}\right) .
\end{gathered}
$$

Considering the $n$th receiving element on the surface in Figure 2.3, it can be said that, the second term in the right-hand side of (2.41) represents the forward propagating field contribution due to the radiation of current elements in front (elements where $x<x_{n}$ ) of the receiving element. Likewise, the term on the right-hand side of (2.42) represents the backward propagating field contribution due to the radiation of current elements in the rear (elements where $x>x_{n}$ ) of the receiving element. Therefore, (2.41) and (2.42) may be defined as the forward propagation and backward propagation equations, respectively.

The total induced current on the $n$th receiving element is composed of the sum of the forward $\left(\mathbf{I}^{f}\right)$ and backward $\left(\mathbf{I}^{b}\right)$ field-induced currents. An iterative procedure can be used to solve forward and backward propagation equations by initializing $\mathbf{I}^{b, 0}=0$, and at the $q$ th sweep,

$$
\left(\overline{\mathbf{Z}}^{s}+\overline{\mathbf{Z}}^{f}\right) \cdot \mathbf{I}^{f,(q)}=\mathbf{V}-\overline{\mathbf{Z}}^{f} \cdot \mathbf{I}^{b,(q-1)}
$$




$$
\left(\overline{\mathbf{Z}}^{s}+\overline{\mathbf{Z}}^{b}\right) \cdot \mathbf{I}^{b,(q)}=-\overline{\mathbf{Z}}^{b} \cdot \mathbf{I}^{f,(q)}
$$

Since $\overline{\mathbf{Z}}^{s}+\overline{\mathbf{Z}}^{b}$ is an upper triangular matrix and $\overline{\mathbf{Z}}^{s}+\overline{\mathbf{Z}}^{f}$ is a lower triangular matrix, the matrices in this iterative process do not need to be factorized or inverted. Thus, (2.43) and (2.44) can be solved for $\mathbf{I}^{f,(q)}$ and $\mathbf{I}^{b,(q)}$ by forward and backward substitution, respectively. Iterations are continued until surface currents show convergence to within a specified accuracy criterion. The ForwardBackward Method presents very fast convergence within a few iterations. Using FBM, there is no need to store the elements of the impedance matrix, because of the sweeping procedure. However, the surface height data, incident field values at matching points, and forward, backward and total currents have to be stored in $N$ element arrays, where $N$ is the surface unknowns. Therefore, the memory requirement of the method is $O(N)$. The mutual impedance values are recomputed at each iteration with a computational cost of $O\left(Q N^{2}\right)$, where $Q$ is the number of iterations. Since the method obtains very accurate results in a few iterations (usually $Q$ is less than 10), the total computational requirement of the method becomes $O\left(N^{2}\right)$ for large $N$.

The FBM algorithm is a stationary iterative process and, in fact, mathematically equivalent to the well-known symmetric successive over relaxation SSOR iteration [25]. This method is very good at obtaining accurate results, when the matrix in the linear equation system is diagonally dominant. Changing the order of current elements disturbs the diagonally dominant nature, which then strongly affects the convergence of the method. The algorithm may become unstable for re-entrant surfaces where current elements are not numbered sequentially as a function of increasing $x$ coordinate. This limitation of the FBM has been overcome by the Generalized Forward-Backward Method (GFBM), which is a hybrid method based on a combination of the conventional FBM with MoM. In the next section, the convergence and accuracy performance of the ForwardBackward Method is presented with numerical results and comparisons. Also, limitations of the method are investigated. 


\subsection{Numerical Results for the FBM}

In this section, numerical results are presented to validate the convergence and accuracy of the Forward-Backward Method over different one-dimensional surface profiles. Results are obtained for both horizontal and vertical polarizations, considering the profiles representing both perfect and imperfect electric conductor surfaces.

In order to check the accuracy of the method, results are compared with the Method of Moments. The residual error is used for monitoring the convergence of the FBM in terms of the number of iterations. The residual error vector after the $q$ th iteration is defined as

$$
\mathbf{r}^{(q)}=\mathbf{V}-\overline{\mathbf{Z}} \cdot \mathbf{I}^{(q)}
$$

Substituting (2.43) and (2.44) into (2.45), the residual error vector can be evaluated in a more efficient way as

$$
\mathbf{r}^{(q)}=\overline{\mathbf{Z}}^{f} \cdot\left[\mathbf{I}^{(q-1)}-\mathbf{I}^{(q)}\right]
$$

The residual error is defined as

$$
\text { residual error }=\frac{\left\|\mathbf{r}^{(q)}\right\|}{\|\mathbf{V}\|}
$$

where $\left\|\mathbf{r}^{(q)}\right\|$ denotes the vector norm. Another convergence criterion, the absolute error of the FBM method, which is defined by

$$
\text { absolute error }=\frac{\left\|\mathbf{I}^{(q)}-\mathbf{I}_{M o M}\right\|}{\left\|\mathbf{I}_{M o M}\right\|}
$$

is also used to check the accuracy.

The results are grouped according to the type of surface profiles. Studies of the FBM over strip profiles and rough surfaces are examined separately in Sections 2.3.1 and 2.3.2, respectively. Operating frequency is chosen to be 300 $\mathrm{MHz}$ for all results in this Chapter. 


\subsubsection{Applications of the FBM over Strip Profiles}

Figure 2.4 shows a strip profile $z=f(x)=0$, which is illuminated by an electromagnetic source, and its discretization into $N$ segments with pulse basis functions. Applying point matching, the matrix equation to be solved for unknown coefficients of current pulses is obtained as

$$
\mathbf{V}=\overline{\mathbf{Z}} \cdot \mathbf{I}
$$

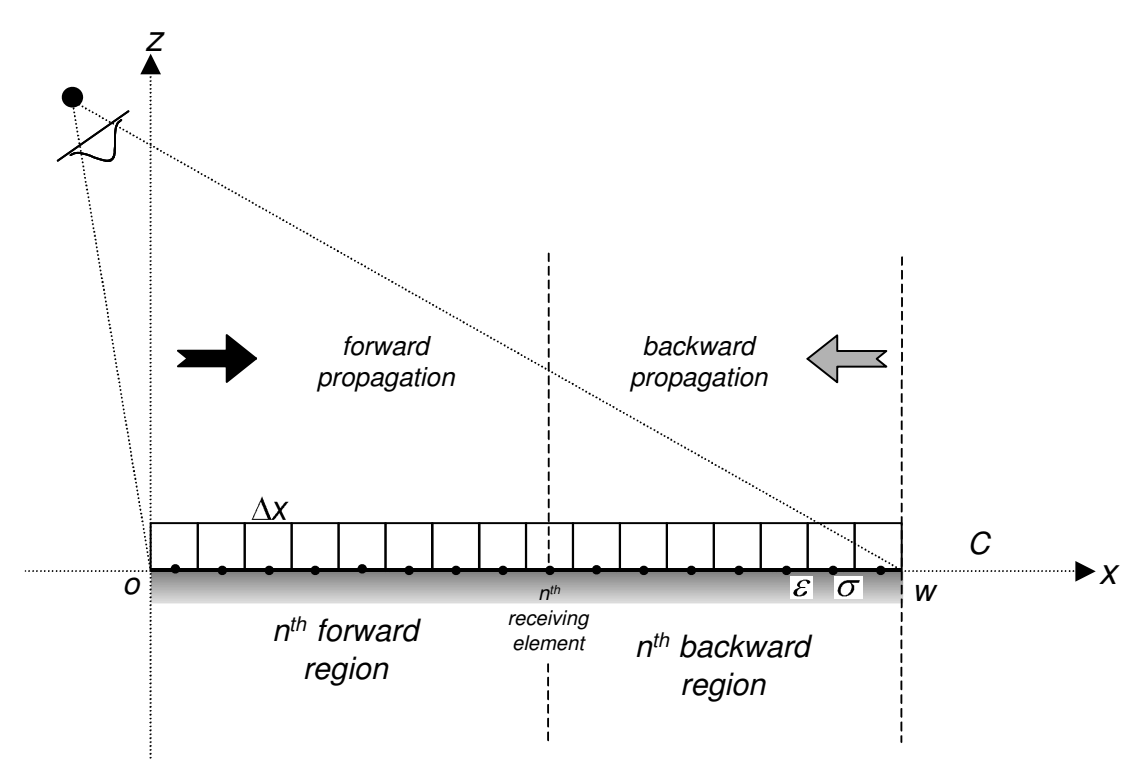

Figure 2.4: One-dimensional strip profile

The first surface is a strip profile with a width of $w=50 \lambda$, excited by a finite plane wave having a horizontal polarization. Taking the segment length $\Delta x=\frac{\lambda}{10}$, the strip profile can be discretized into $N=500$ segments. Using (2.18) and (2.19), the entries of the impedance matrix are evaluated. Since the incident field is a finite plane wave, elements of the vector $\mathbf{V}$ become

$$
V_{n}=-E_{y}^{i n c}\left(\boldsymbol{\rho}_{n}\right)= \begin{cases}e^{-j k\left(x_{n} \cos \theta-z_{n} \sin \theta\right)} & , \text { if } 0<x_{n}<w \\ 0 & , \text { otherwise }\end{cases}
$$

where $\theta$ is the angle of incidence from $x$-axis. The normal and grazing incidence cases are considered taking the incidence angle $\theta=\frac{\pi}{2}$ and $\theta=\frac{\pi}{20}$, respectively. 


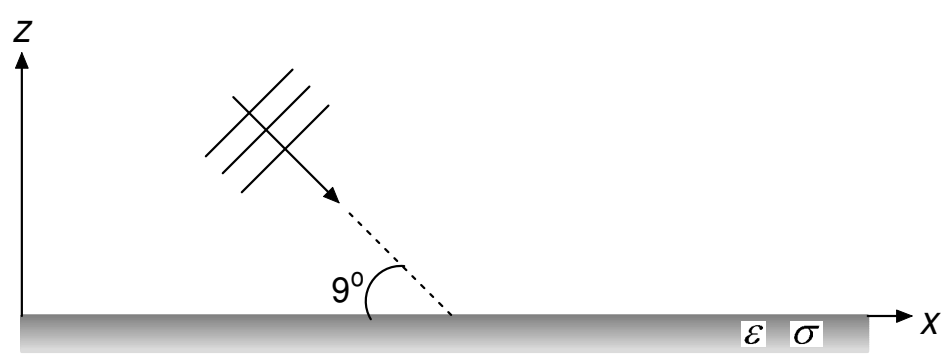

Figure 2.5: Strip profile excited by a grazing incident plane wave

Then, the MoM solution for unknown induced currents can be found by solving the matrix equation in (2.49) via Gaussian elimination method as

$$
\mathbf{I}=\overline{\mathbf{Z}}^{-1} \cdot \mathbf{V}
$$

In order to validate the accuracy of the Forward-Backward Method with this geometry, the induced current obtained with FBM, using iterative forward and backward radiation equations (2.43) and (2.44), is compared with the reference solution given by MoM applied to the whole surface for both normal and grazing cases of incidence.

Figure 2.6 (a) and (b) illustrate the comparison of induced currents obtained

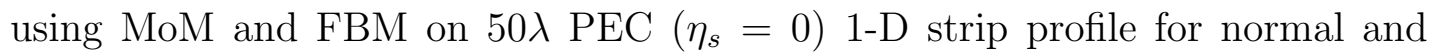
grazing incident plane wave cases, respectively, while (c) and (d) show the same comparison on $50 \lambda$ non-PEC $\left(\eta_{s}=15+j 20 \Omega\right)$ strip. It is obvious that the FBM yields very accurate results for both normal and grazing incidence cases on PEC and non-PEC strip profiles.

In order to show the accuracy of the method, the residual and absolute error versus the number of iteration graphs are illustrated in Figure 2.7. It is observed that, after six or seven iterations, the values converge to an accuracy level of residual error about $10^{-3}$. It should be noted that the method gives more accurate results for the normal incidence case than the grazing incident case. Also, error values for studies over non-PEC surfaces are less than those of PEC surfaces. This 
(a) Normal Incident Planewave on PEC Strip

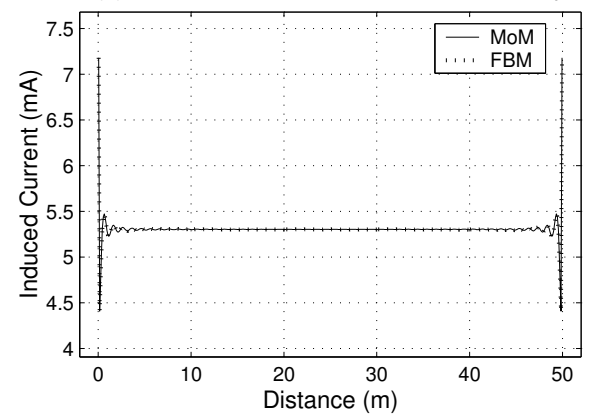

(c) Normal Incident Planewave on non-PEC Strip

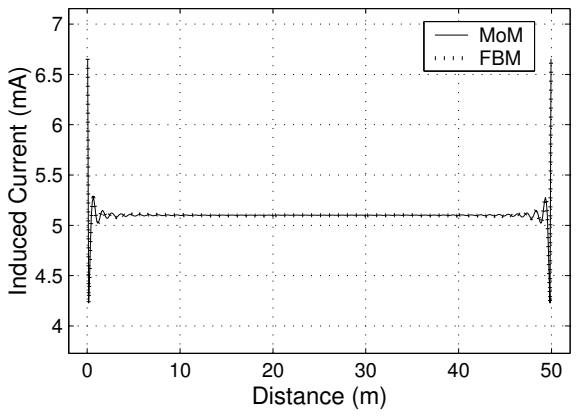

(b) Grazing Incident Planewave on PEC Strip

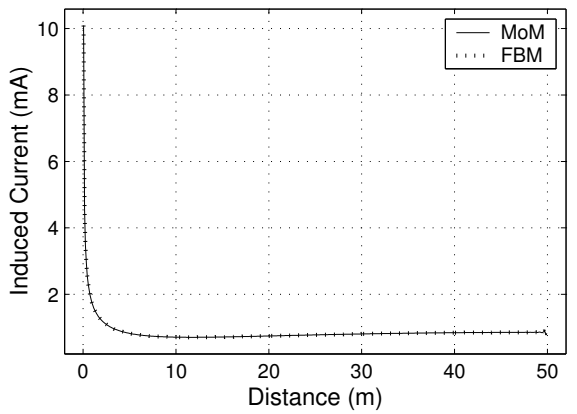

(d) Grazing Incident Planewave on non-PEC Strip

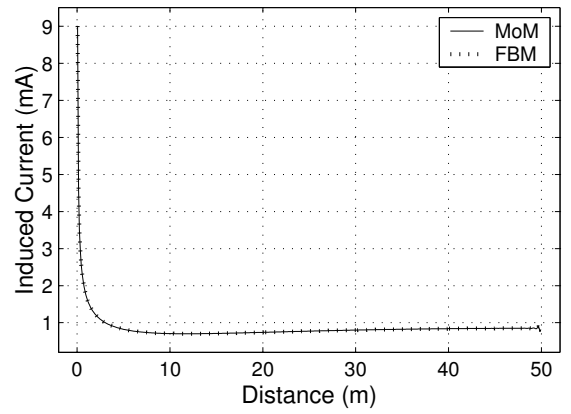

Figure 2.6: Induced current on a $50 \lambda$ strip (TM Pol.)
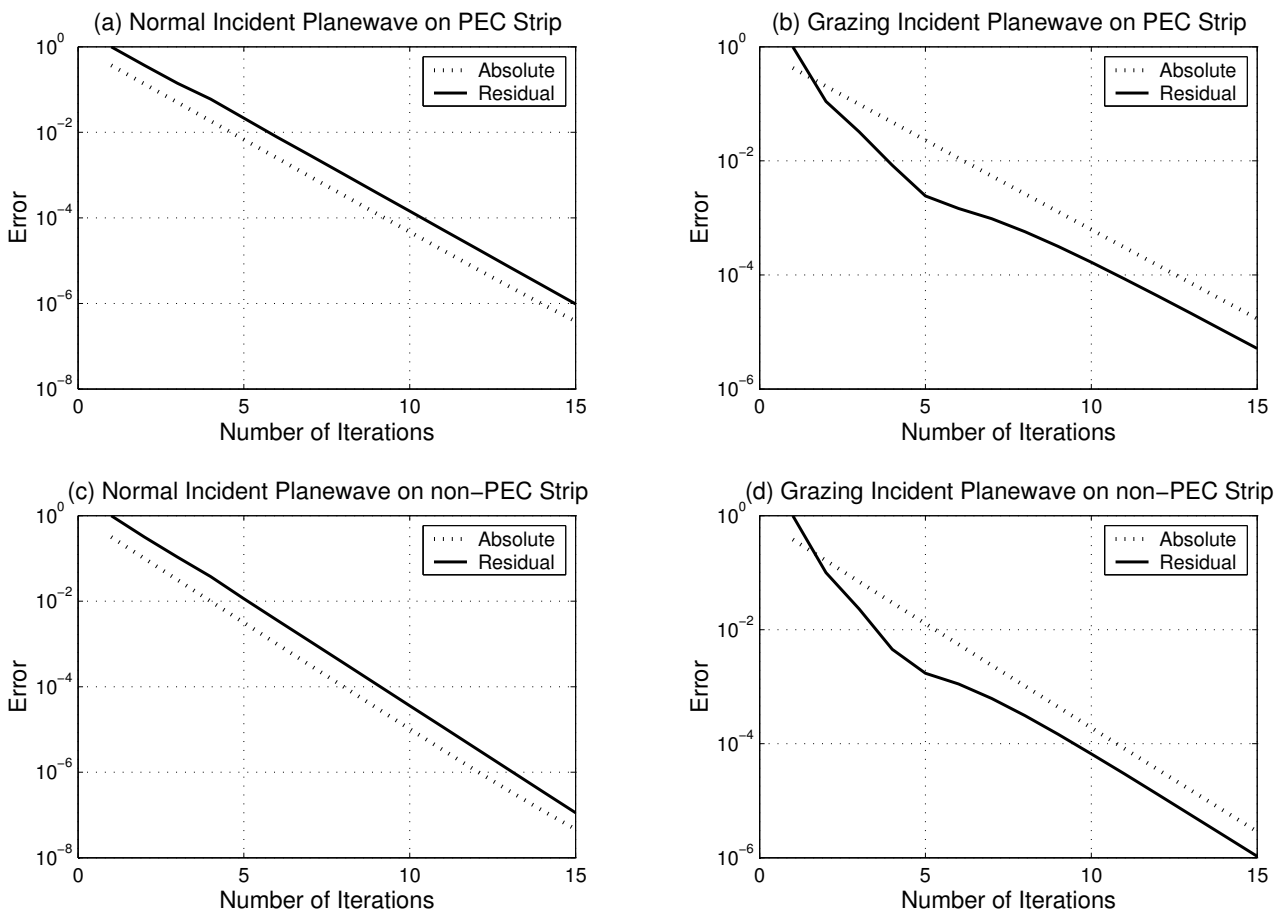

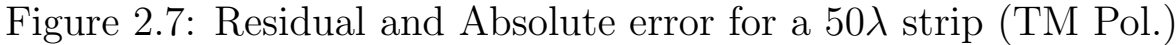


is because of the non-zero surface impedance term in the expression for diagonal elements of the impedance matrix, which is given in (2.19). Since self terms have larger amplitudes for the non-PEC case, the matrix becomes diagonally more dominant, and hence the FBM gives more accurate results for the non-PEC case.

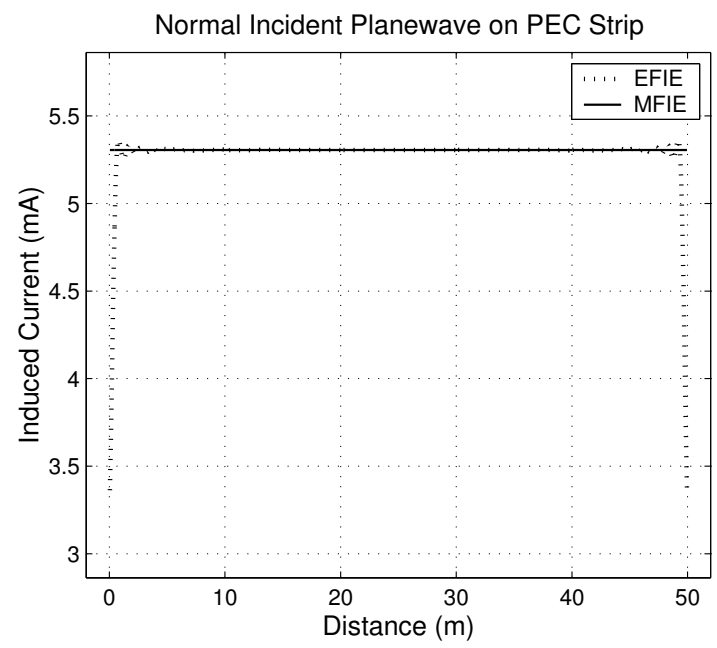

Figure 2.8: Induced Current on a strip (TE Pol.)

If the plane wave radiating over the strip is vertically polarized, (2.34) and (2.35) must be used to evaluate the mutual and self interactions. For the PEC case, the terms including the surface impedance vanish, because $\eta_{m}=0$. Also, since the surface is a strip profile of $z=f(x)=0$, the dot product term disappears, since $\hat{\rho}_{n m}$ is perpendicular to surface normal for any pairs of source and observation points. Thus, the self and mutual interactions are found as:

$$
Z_{n m}=\left\{\begin{array}{cc}
\frac{1}{2} & , m=n \\
0 & , m \neq n
\end{array}\right.
$$

and this results in

$$
J_{t}(\boldsymbol{\rho})=-2 H_{y}^{i n c}(\boldsymbol{\rho})
$$

This is consistent with the assumption of considering the surface profile extended to infinity, because of the fact, the induced current on an infinite strip is the physical optics current $\mathbf{J}_{s}^{P O}=2 \hat{n} \times \mathbf{H}^{i n c}$. This prevents to reach the numerically 
accurate solution of the induced current over strip profiles for vertical polarization case. However, since the dot product term does not vanish for rough surface profiles, the MFIE formulation has been generally used for vertical polarized incident field.

Figure 2.8 illustrates the induced current over 50 $\lambda$ PEC strip, which is illuminated by a vertical polarized normal incident plane wave. The amplitude of the magnetic field is taken as $\frac{1}{\eta_{0}}$. The induced current obtained using magnetic and electric field integral equations are compared. Since the EFIE formulation for vertical polarization case is not as computationally efficient as the MFIE formulation, the MFIE formulation is generally used for computing the scattered fields over electrically large rough surface profiles.

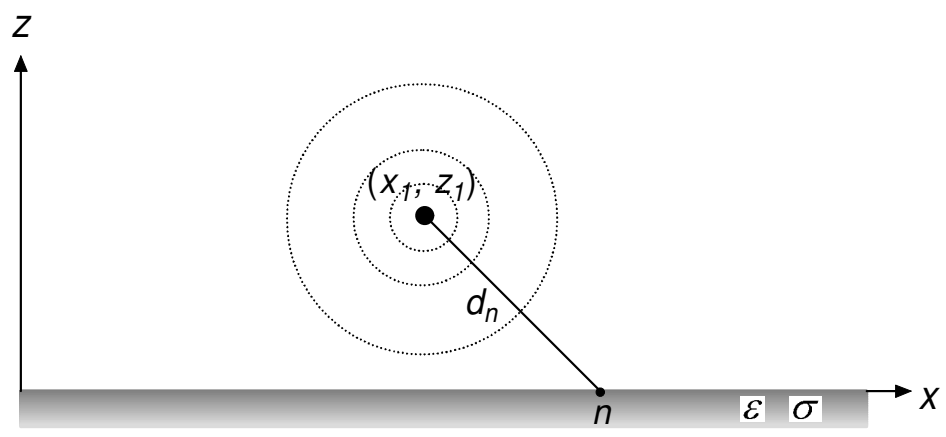

Figure 2.9: Isotropic radiator on a $100 \lambda$ strip

Consider the geometry illustrated in Figure 2.9. The strip having a width of $100 \lambda$ is symmetrically illuminated by an electromagnetic source located at the coordinates $\left(x_{1}=50 \lambda, z_{1}=25 \lambda\right)$. Assuming the source to be an isotropic radiator, and considering a horizontal polarized cut of its spherical radiation pattern on the $x z$-plane, the elements of the excitation vector can be given as,

$$
V_{n}=-E_{y}^{i n c}\left(\boldsymbol{\rho}_{n}\right)=-E_{0} \frac{e^{-j k d_{n}}}{d_{n}}
$$

where

$$
d_{n}=\sqrt{\left[x_{n}-x_{1}\right]^{2}+\left[z_{n}-z_{1}\right]^{2}} .
$$


Using the radiation density integral in [39]

$$
P_{t}=\int_{\theta=0}^{\pi} \int_{\phi=0}^{2 \pi} \frac{\left|E_{o}\right|^{2}}{2 \eta} \sin \theta d \theta d \phi
$$

the magnitude of the electric field can be related to the transmitted power as

$$
E_{0}=\sqrt{60 P_{t}}
$$

$P_{t}$ denotes the transmitted power from the isotropic radiator in $(2.57)$ and it is considered to be 25 Watts.

Locating the same isotropic radiator to the coordinates $\left(x_{1}=0, z_{1}=25 \lambda\right)$, the non-symmetric incidence case can be considered. In order to validate the accuracy of the Forward-Backward Method with this geometry, the induced current obtained with FBM, is compared with the reference solution given by MoM applied to the whole surface for both symmetric $\left(x_{1}=50 \lambda, z_{1}=25 \lambda\right)$ and non-symmetric $\left(x_{1}=0, z_{1}=25 \lambda\right)$ illumination cases. Figure 2.11 (a) and (b) illustrate the comparison of induced currents obtained using MoM and FBM on 100 $\lambda$ PEC $\left(\eta_{s}=0\right)$ strip for symmetric and non-symmetric incidence cases, respectively, while (c) and (d) show the same comparison on a $100 \lambda$ non-PEC $\left(\eta_{s}=20+j 15 \Omega\right)$ strip. For the symmetric incidence case, the current is also induced symmetrically on the surface profile. Residual and absolute error results in Figure 2.12 show similar characteristics to those of previous geometry. After very few iterations, the accuracy level of residual error about $10^{-3}$ is reached. Better convergence of the current values for the non-PEC surface is observed again, because of relatively larger self terms in the impedance matrix.

Figure 2.10 illustrates another strip profile of $w=200 \lambda$ width. This surface is considered to be illuminated by a horizontally polarized dipole antenna located symmetrically at coordinates $\left(x_{1}=100 \lambda, z_{1}=25 \lambda\right)$. For this type of source, elements of the incident field vector can be given by,

$$
V_{n}=-E_{y}^{i n c}\left(\boldsymbol{\rho}_{n}\right)=-E_{0} \frac{e^{-j k d_{n}}}{d_{n}} \sin \theta_{n}
$$


where $\theta_{n}$ is the elevation angle of the receiving element from the vertical axis of the source, and $\sin \theta_{n}$ can be determined for the strip geometry by,

$$
\sin \theta_{n}=\frac{x_{n}-x_{1}}{d_{n}}
$$

and

$$
E_{0}=\sqrt{90 P_{t}}
$$

is found using (2.56). $d_{n}$ denotes the distance between the $n$th receiving element and the source, and it is given by (2.55). $P_{t}$ is the transmitted power from the horizontally polarized dipole antenna, which is considered to be 25 Watts. Choosing the pulse width as $\Delta x=\frac{\lambda}{10}$, the strip profile can be discretized into $N=2000$ segments.

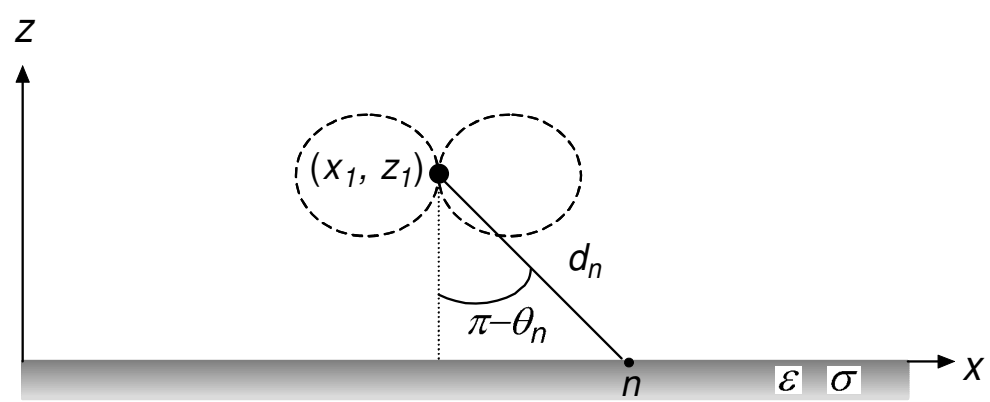

Figure 2.10: Infinitesimal Dipole on a $200 \lambda$ strip

The non-symmetric incidence case can be considered, changing the coordinates of the same dipole antenna to $\left(x_{1}=0, z_{1}=25 \lambda\right)$. In order to test the Forward-Backward Method with this geometry, the FBM current is determined on center points of these segments and compared with the reference solution.

For both symmetric and non-symmetric cases of incidence, Figure 2.13 illustrates that FBM results suit very well to the MoM reference solutions on both $\operatorname{PEC}\left(\eta_{s}=0\right)$ and non-PEC $\left(\eta_{s}=20+j 20 \Omega\right)$ strip profiles of $200 \lambda$ width. In order to show the accuracy of the method, the residual and absolute error versus the number of iteration graphs are illustrated in Figure 2.14. 
(a) Isotropic Rad. on PEC Strip (Symmetric)

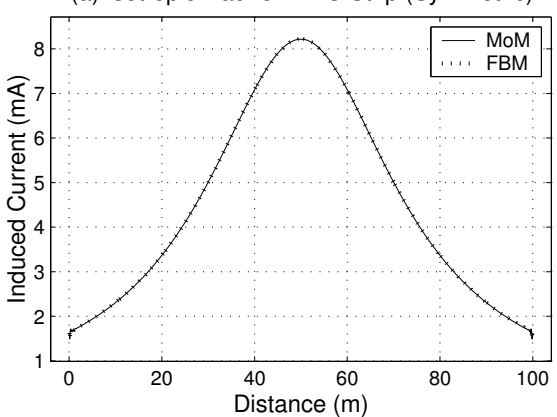

(c) Isotropic Rad. on non-PEC Strip (Symmetric)

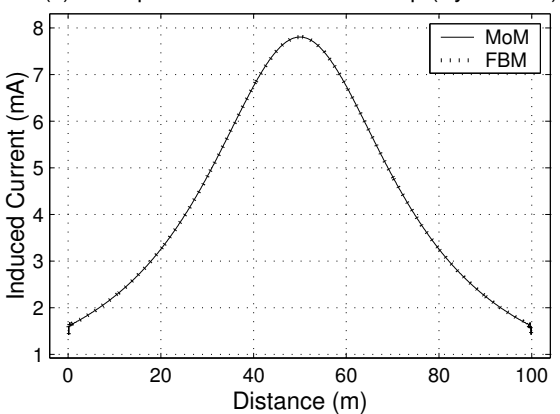

(b) Isotropic Rad. on PEC Strip (non-symmetric)

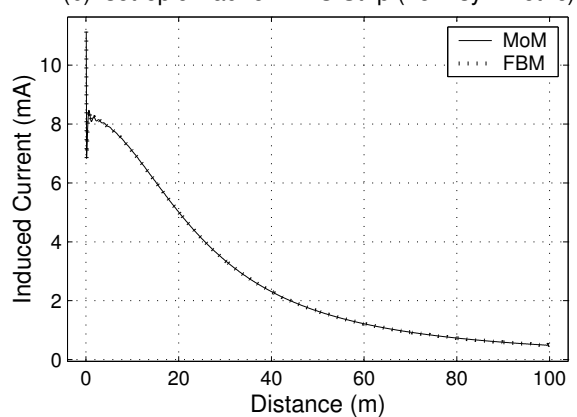

(d) Isotropic Rad. on non-PEC Strip (non-symmetric)

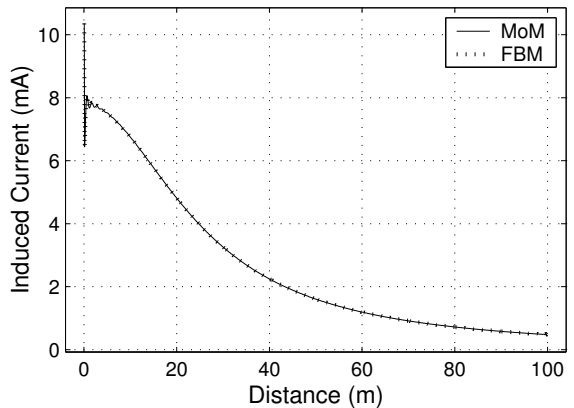

Figure 2.11: Induced current on a $100 \lambda$ strip (TM Pol.)
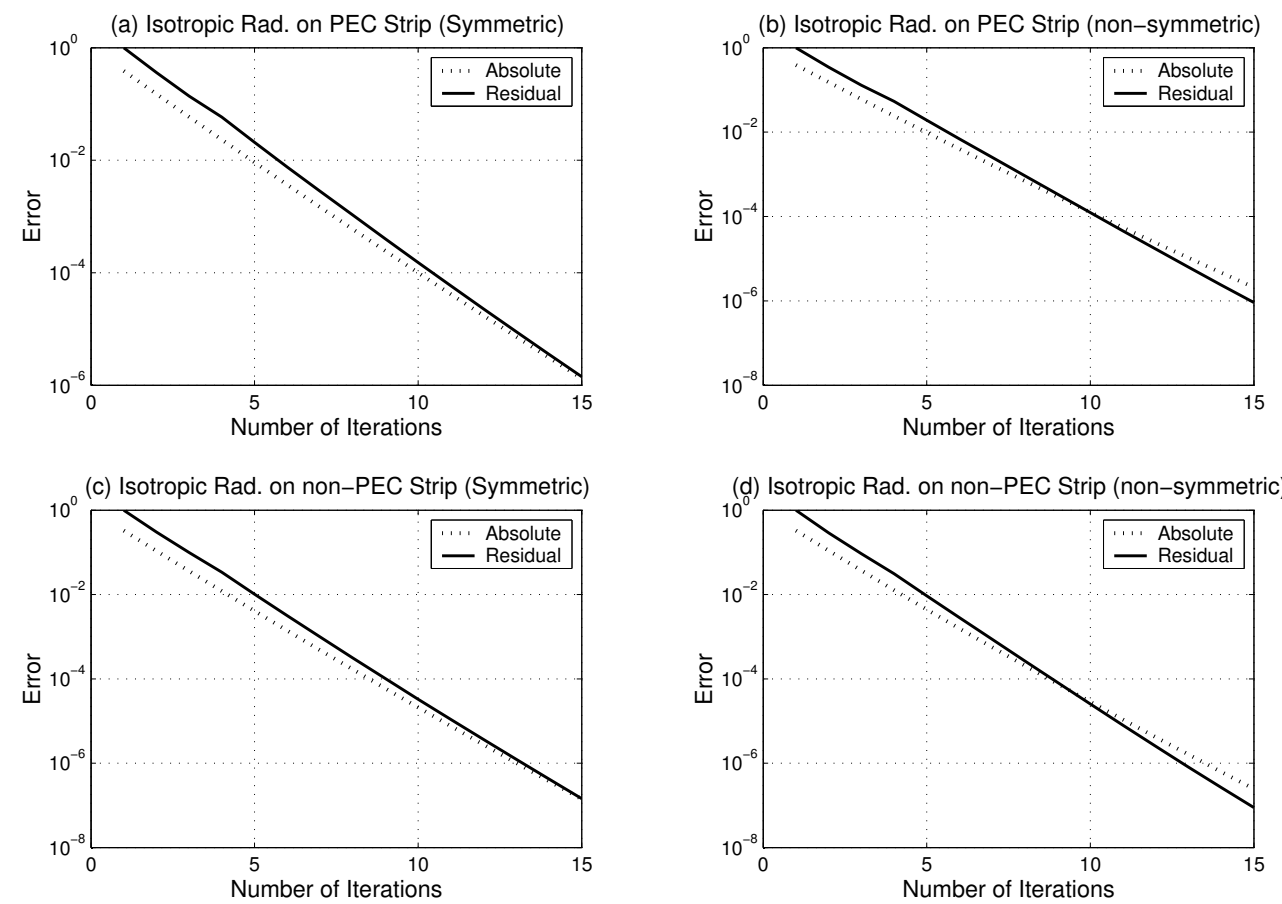

Figure 2.12: Residual and Absolute error for a $100 \lambda$ strip (TM Pol.) 
It is observed that, since self terms have larger amplitudes for the non-PEC case, the matrix becomes diagonally more dominant, and hence the FBM gives more accurate results for non-PEC case.

After 2000 surface unknowns, to obtain the MoM reference solution becomes a very difficult problem because of the $O\left(N^{3}\right)$ computational cost. However, tests of the FBM so far show that the FBM solution can be used as a numerically accurate reference for computing the scattering from strip profiles. As mentioned before, after six or seven iterations, the values converge to an error level about $10^{-3}$.

Consider a strip profile with a width of $w=500 \lambda$, excited by a finite plane wave having a horizontal polarization. The normal and grazing incidence cases are considered taking the incidence angle $\theta=\frac{\pi}{2}$ and $\theta=\frac{\pi}{20}$, respectively. Figure 2.15 (a) and (b) illustrate the induced current obtained using FBM after six iterations on $500 \lambda \operatorname{PEC}\left(\eta_{s}=0\right)$ strip for normal and grazing incident plane wave cases, respectively.

Locating an isotropic radiator at the coordinates $\left(x_{1}=500 \lambda, z_{1}=25 \lambda\right)$ and at $\left(x_{1}=0 z_{1}=25 \lambda\right)$ over a strip profile having a width of $1000 \lambda$ symmetric and non-symmetric cases of incidence are taken into account, respectively. Figure 2.16 (a) and (b) illustrate the induced current obtained using FBM after six iterations on $1000 \lambda$ non-PEC $\left(\eta_{s}=20+j 15 \Omega\right)$ strip for symmetric and non-symmetric incidence cases, respectively.

Finally, consider a strip profile with a width of $w=2000 \lambda$, excited by a dipole antenna having a horizontal polarization. The symmetric and non-symmetric incidence cases are considered locating the source at $\left(x_{1}=1000 \lambda, z_{1}=25 \lambda\right)$ and at $\left(x_{1}=0 z_{1}=25 \lambda\right)$, respectively. Figure 2.17 (a) and (b) illustrate the induced current obtained using FBM after six iterations on $2000 \lambda$ non-PEC $\left(\eta_{s}=\right.$ $20+j 20 \Omega$ ) strip for symmetric and non-symmetric incidence cases, respectively. 
(a) Dipole on PEC Strip (Symmetric)
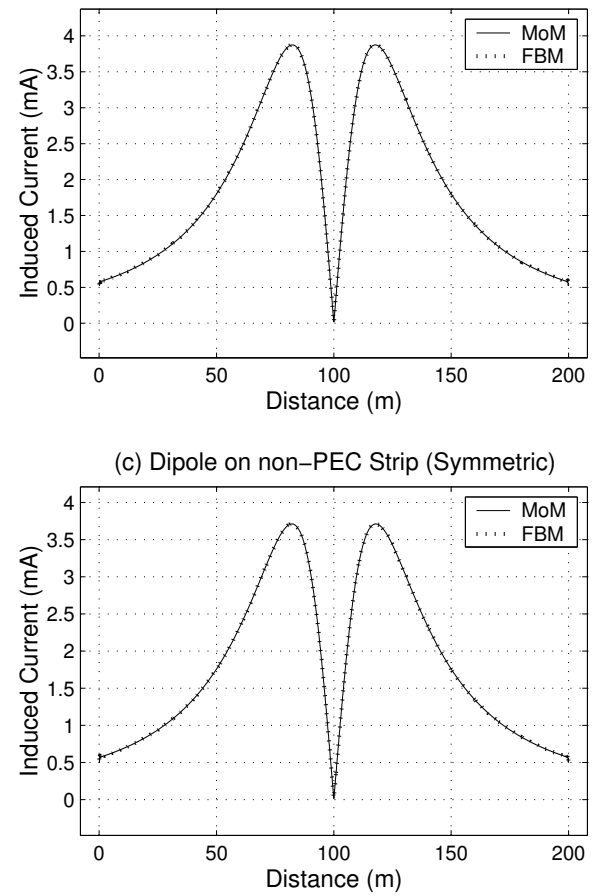

(b) Dipole on PEC Strip (non-symmetric)

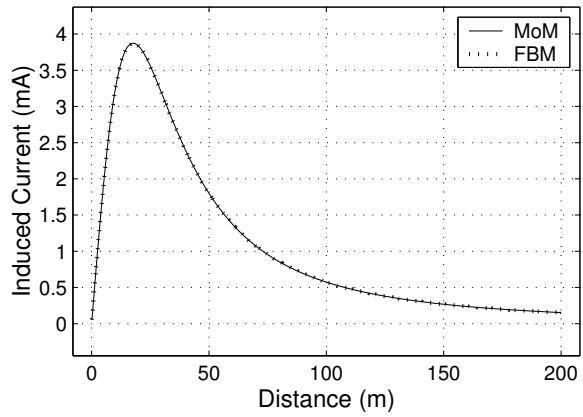

(d) Dipole on non-PEC Strip (non-symmetric)

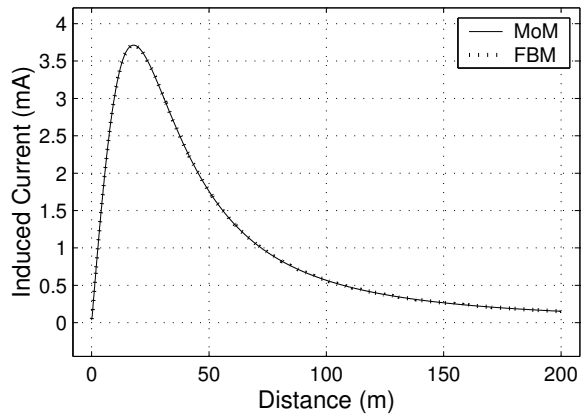

Figure 2.13: Induced current on a $200 \lambda$ strip (TM Pol.)
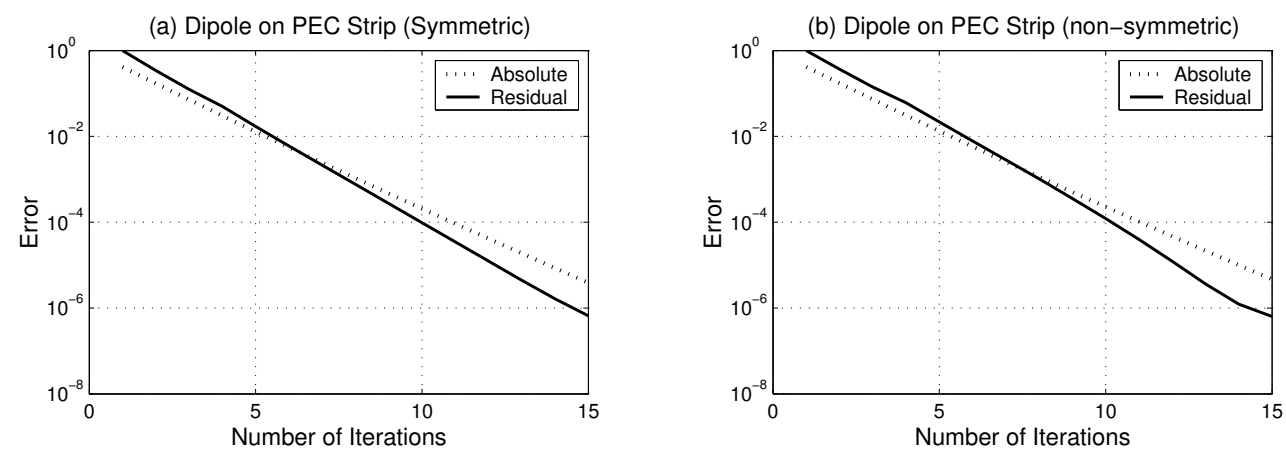

(c) Dipole on non-PEC Strip (Symmetric)
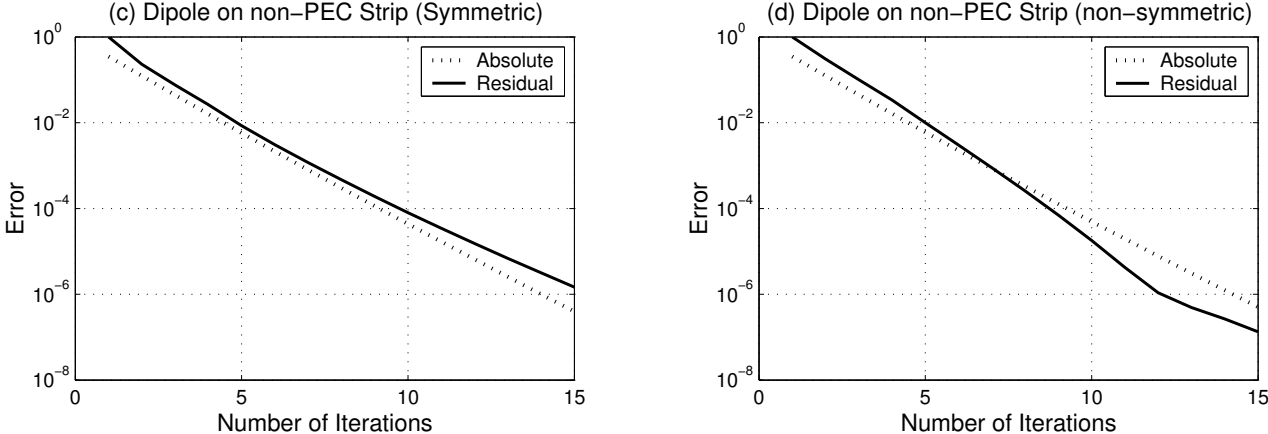

Figure 2.14: Residual and Absolute error for a 200 $\lambda$ strip (TM Pol.) 
(a) Normal Incident Planewave on PEC Strip

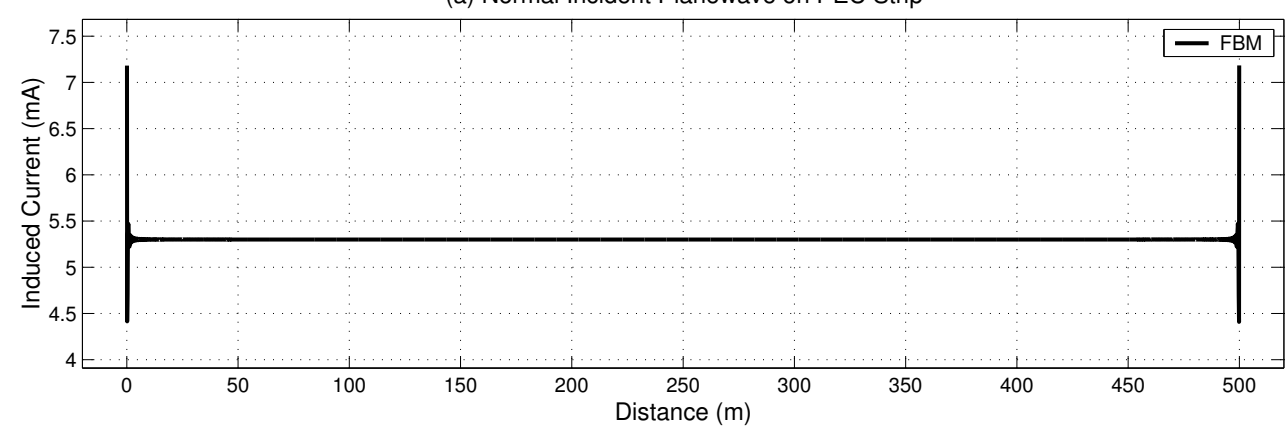

(b) Grazing Incident Planewave on PEC Strip

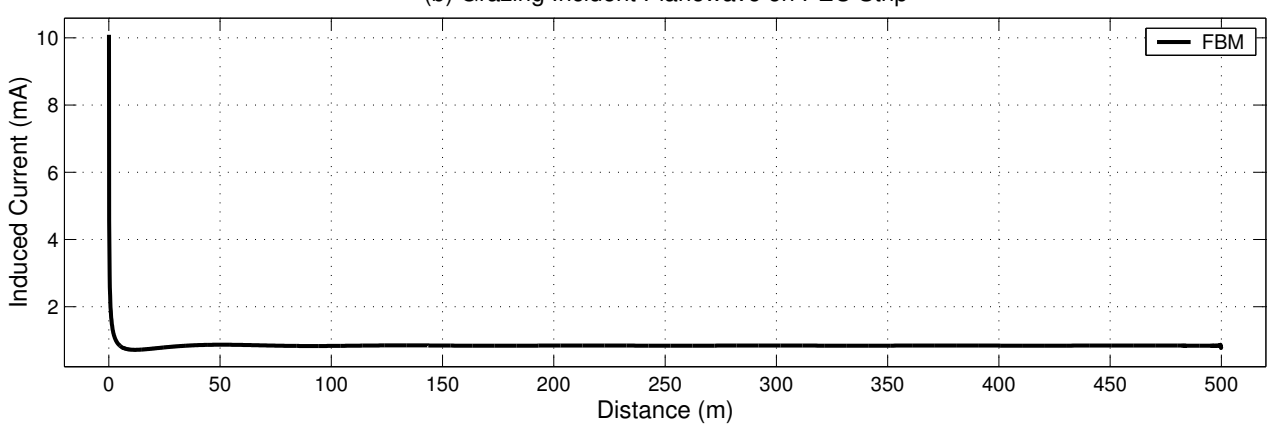

Figure 2.15: Induced current on a 500 $\lambda$ strip (TM Pol.)

(a) Isotropic Radiator on non-PEC Strip (Symmetric)

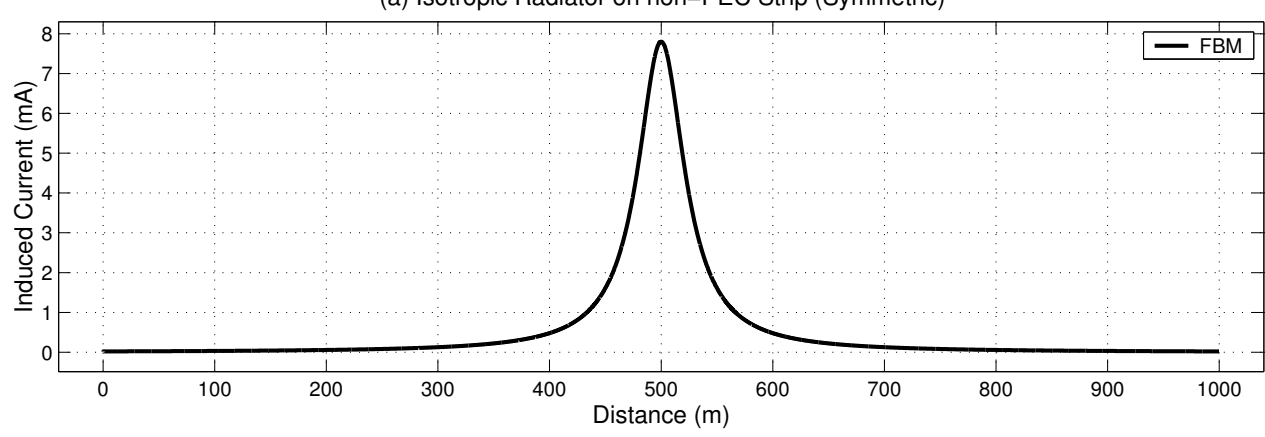

(b) Isotropic Radiator on non-PEC Strip (non-symmetric)

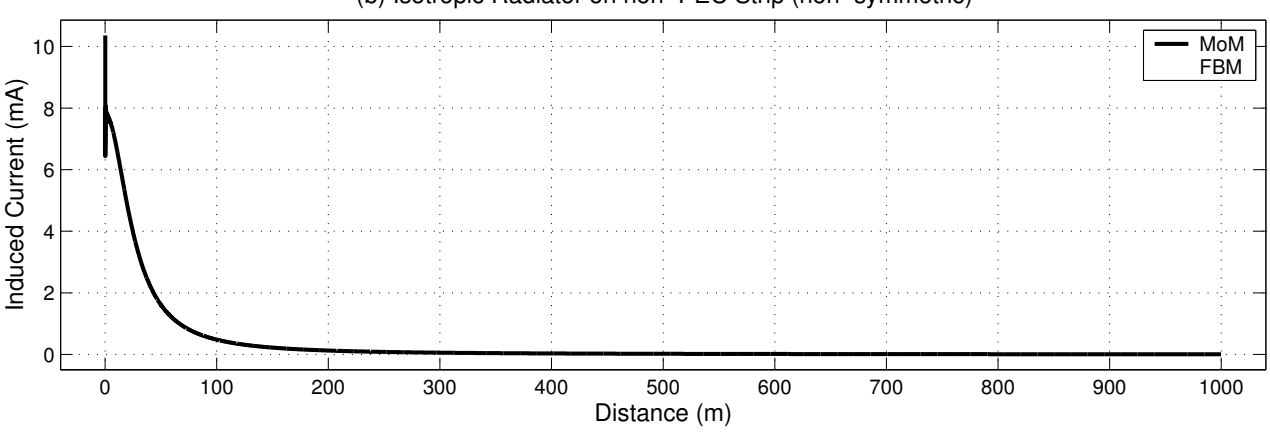

Figure 2.16: Induced current on a $1000 \lambda$ strip (TM Pol.) 
(a) Dipole on non-PEC Strip (Symmetric)

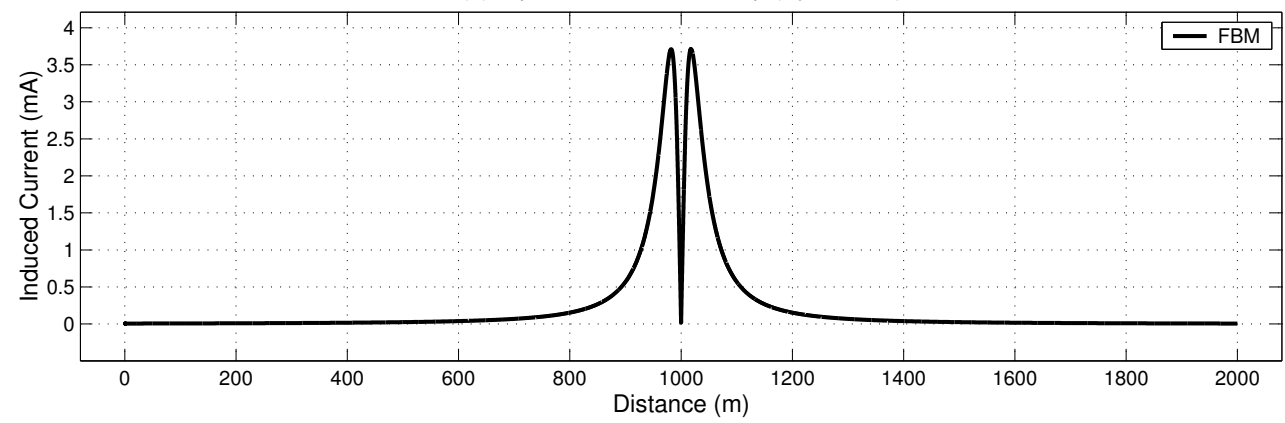

(b) Dipole on non-PEC Strip (non-symmetric)

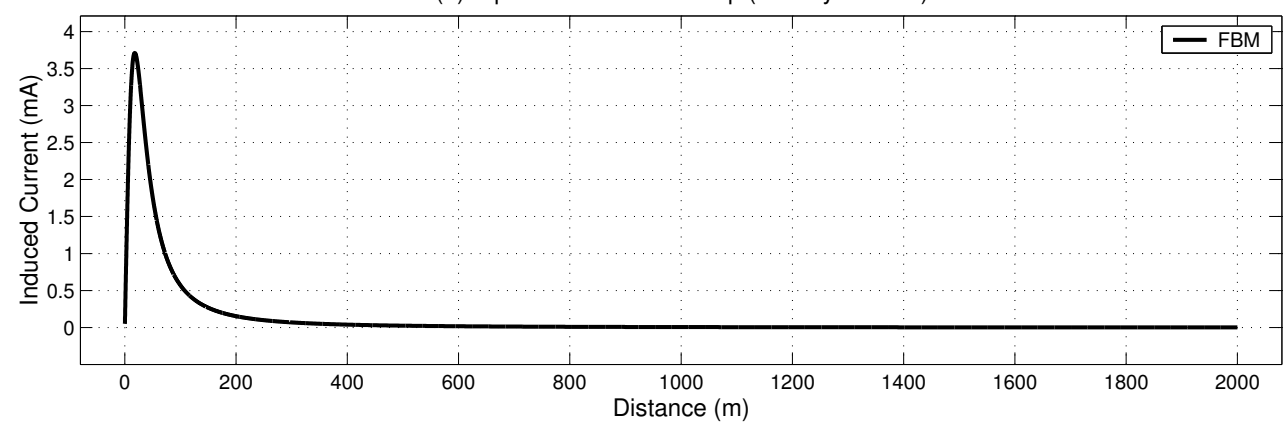

Figure 2.17: Induced current on a 2000 $\lambda$ strip (TM Pol.)

Investigations over strip profiles show that the FBM obtains numerically accurate solutions within very few iterations on strip profiles for horizontal polarization case.

\begin{tabular}{|c|c|c|c|}
\hline$N$ & Matrix Fill (s) & LU Inversion (s) & FBM (s) \\
\hline 500 & 1.5 & 61.5 & 9 \\
1000 & 7 & 547 & 36 \\
2000 & 33.5 & 5981 & 144 \\
5000 & 237 & NA & 90.5 \\
10000 & 989.5 & NA & 393.1 \\
20000 & 4044.5 & NA & 1666.6 \\
\hline
\end{tabular}

Table 2.1: Computational Cost

In order to compare the computational cost of the method with MoM, the computation time versus number of iterations examinations are illustrated in Table 2.1 and in Figure 2.18 for both of the reference methods in the case of horizontally polarized incident field. The continuous line shows the cpu-time for the FBM after six iterations, since after six or seven iterations, the values 


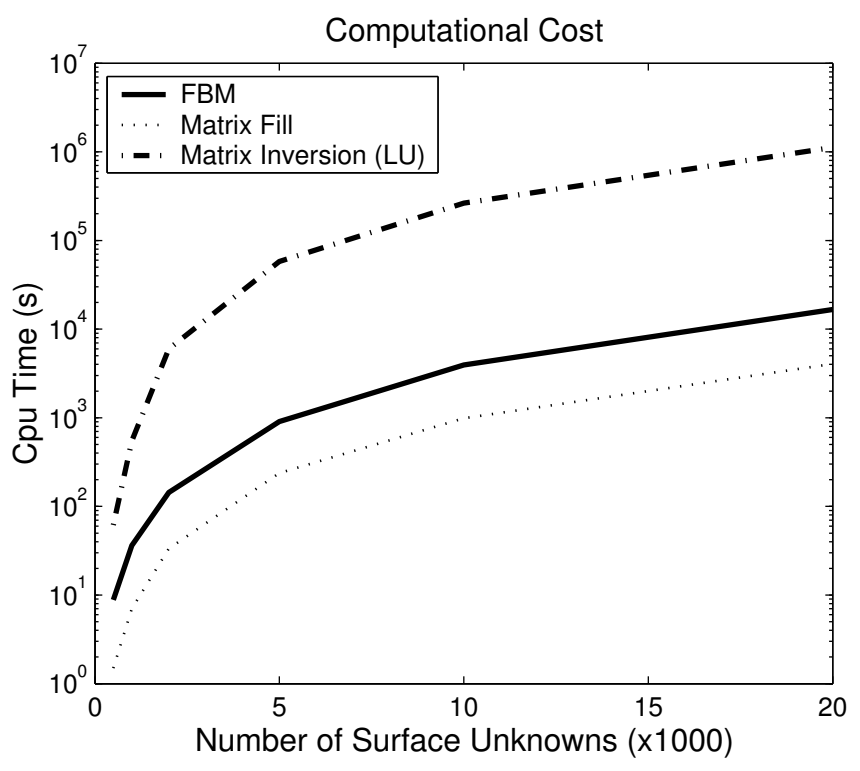

Figure 2.18: Computational Cost

converge to an error level about $10^{-3}$. Computational cost for the matrix filling and direct inversion (LU) for the MoM are illustrated, too. Note that, after 2000 unknowns, cpu-time values for the MoM are obtained using a spline extrapolation of those for less than 2000.

It is obvious that, the FBM presents a computational cost of $O\left(N^{2}\right)$ while the direct solution of the MoM requires $O\left(N^{3}\right)$ cpu-time, where $N$ is the total number of surface unknowns.

The investigations and comparisons demonstrate that, the FBM can be used as a numerically accurate reference solution instead of MoM over strip profiles in horizontal polarization case, because of its very accurate results within very few iterations, causing a computational time of $O\left(N^{2}\right)$ and a memory requirement of $O(N)$. 


\subsubsection{Applications of the FBM over Rough Surface Pro- files}

Figure 2.19 (a) shows a $w=50 \lambda$ symmetric PEC rough surface profile, which is illuminated by a normal incident finite plane wave. Applying point matching with pulse basis functions, the matrix equation to be solved for unknown coefficients of current pulses is obtained as

$$
\mathbf{V}=\overline{\mathbf{Z}} \cdot \mathbf{I}
$$

Assuming a horizontal polarization, using (2.18) and (2.19), the entries of the impedance matrix are evaluated. (2.50) is valid for computing the elements of $V_{n}$ for the horizontal polarization case.

In the case of vertical polarization, the impedance matrix elements are obtained using (2.34) and (2.35). Elements of the vector $\mathbf{V}$ are considered to be

$$
V_{n}=-H_{y}^{i n c}\left(\boldsymbol{\rho}_{n}\right)=\frac{1}{\eta_{o}} \begin{cases}e^{-j k\left(x_{n} \cos \theta-z_{n} \sin \theta\right)} & , \text { if } 0<x_{n}<w \\ 0 & , \text { otherwise }\end{cases}
$$

for the consistency in the amplitude of the induced current, where $\theta$ is the angle of incidence from $x$-axis. The normal incidence case is considered taking the incidence angle $\theta=\frac{\pi}{2}$.

Then, the MoM solution for unknown induced currents can be found by solving the matrix equation in (2.49) via Gaussian elimination method as

$$
\mathbf{I}=\overline{\mathbf{Z}}^{-1} \cdot \mathbf{V}
$$

In order to validate the accuracy of the Forward-Backward Method with this geometry, the induced current obtained with FBM, using iterative forward and backward radiation equations (2.43) and (2.44), is compared with the reference solution given by MoM applied to the whole surface for both horizontal and vertical cases of incidence. 
(a) PEC Rough Surface Profile

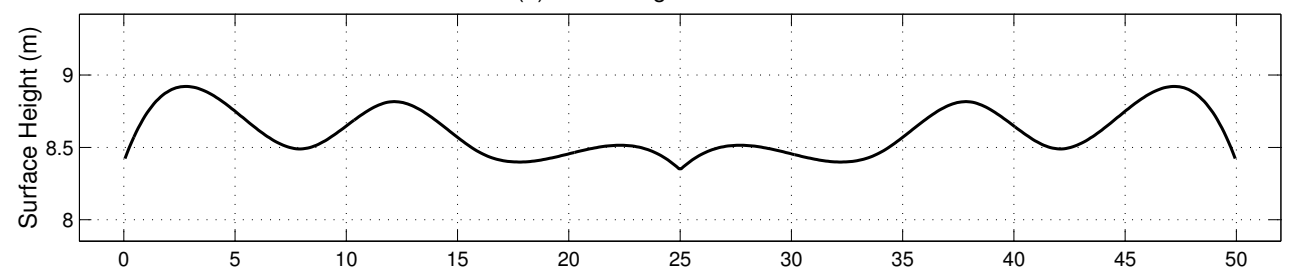

(b) TM Polarized Planewave on PEC Surface (Normal)

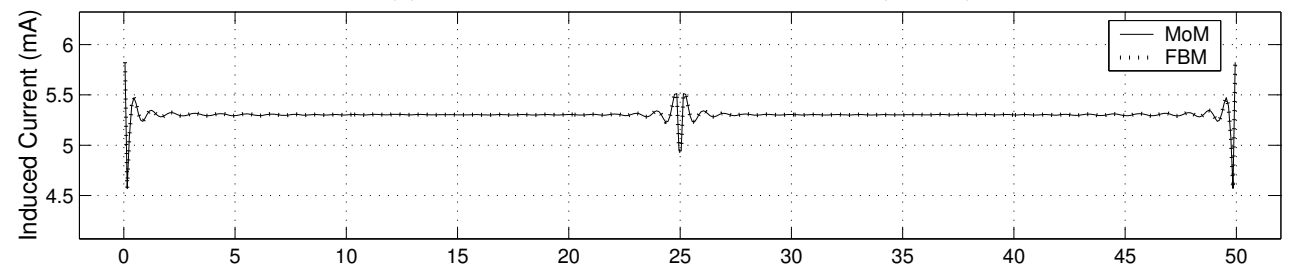

(c) TE Polarized Planewave on PEC Surface (Normal)

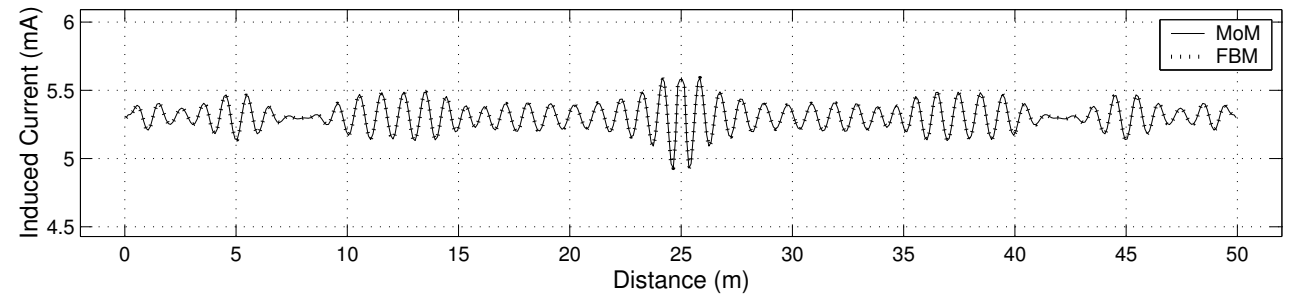

Figure 2.19: Induced current on a $50 \lambda$ rough surface

Figure 2.19 (b) and (c) illustrate the comparison of induced currents obtained using MoM and FBM on 50ג PEC $\left(\eta_{s}=0\right)$ rough surface profile for normal incident TM and TE polarized plane wave cases, respectively.

The grazing incidence case is considered taking the incidence angle $\theta=\frac{\pi}{20}$. Figure 2.20 (b) and (c) illustrate the comparison of induced currents obtained using MoM and FBM for grazing incident TM and TE polarized plane wave cases, respectively, on the same $50 \lambda \mathrm{PEC}\left(\eta_{s}=0\right)$ rough surface profile.

It is obvious that the FBM presents very accurate results in both normal and grazing incidence cases for TM and TE polarizations. Especially the effects of shadowed regions on the induced current are clearly observed for the grazing incidence case. In order to show the accuracy of the method, the residual and absolute error versus the number of iteration graphs are illustrated in Figure 2.21. As mentioned before, after six or seven iterations, the values converge to an error level about $10^{-3}$ in the case of horizontal polarization. In the vertical 
(a) PEC Rough Surface Profile

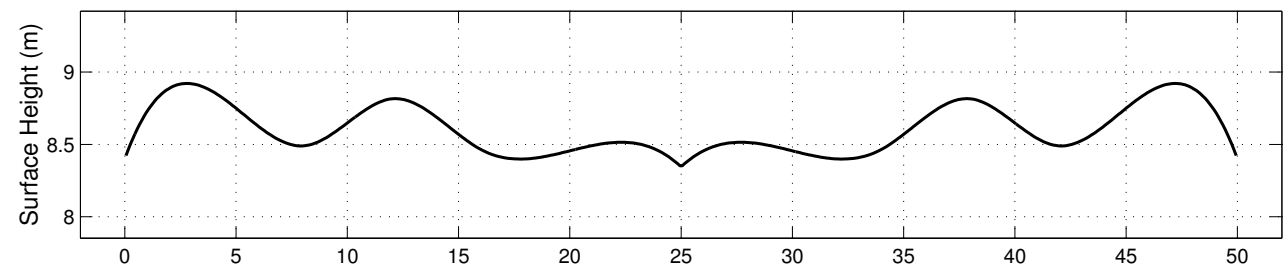

(b) TM Polarized Planewave on PEC Surface (Grazing)

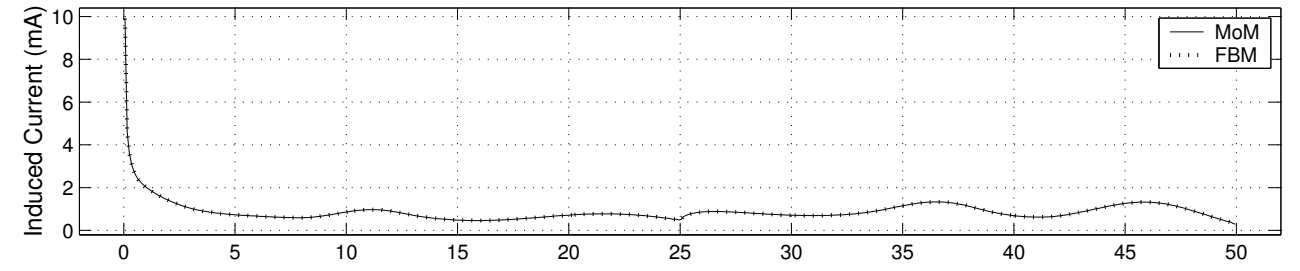

(c) TE Polarized Planewave on PEC Surface (Grazing)

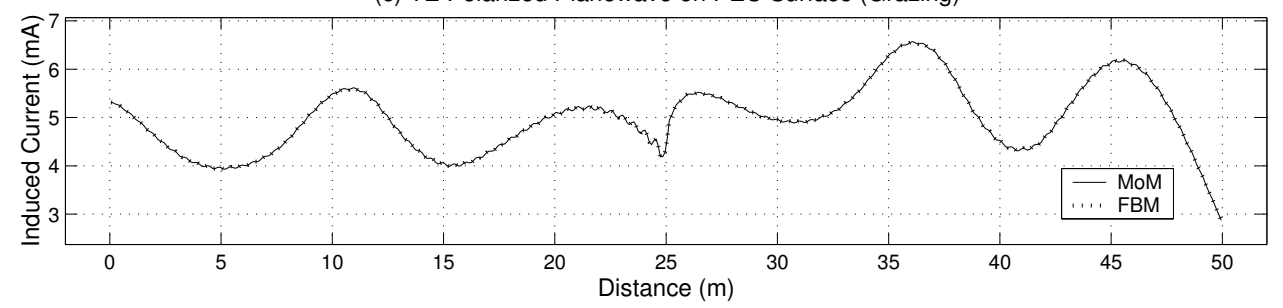

Figure 2.20: Induced current on a $50 \lambda$ rough surface
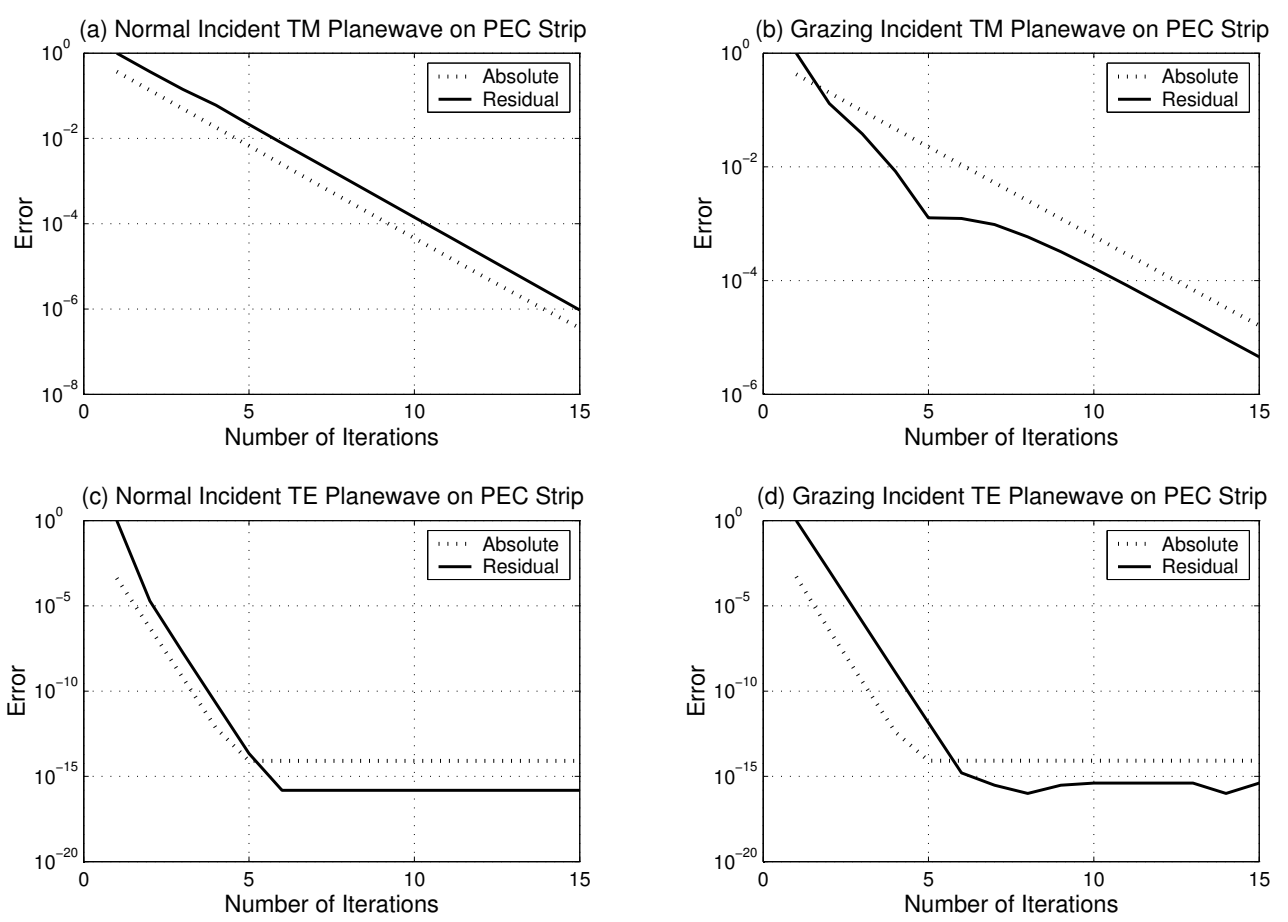

Figure 2.21: Residual and Absolute error for a $50 \lambda$ rough surface 
polarization case the required number of iterations to reach a desirable error level is two. The impedance matrix in the TE case is much more diagonally dominant than that in the TM case, so that the FBM presents more accurate results within much less number of iterations in the TE case.

\section{Isotropic Radiator on a $100 \lambda$ rough surface}

Figure 2.22 (a) shows a $w=100 \lambda$ symmetric non-PEC rough surface profile, which is illuminated by an isotropic radiator located symmetrically over the surface at a height above $25 \lambda$ in the middle of the surface. Assuming a horizontal polarization, using (2.18) and (2.19), the entries of the impedance matrix are evaluated. (2.54)-(2.57) are valid for computing the elements of $V_{n}$ for the horizontal polarization case.

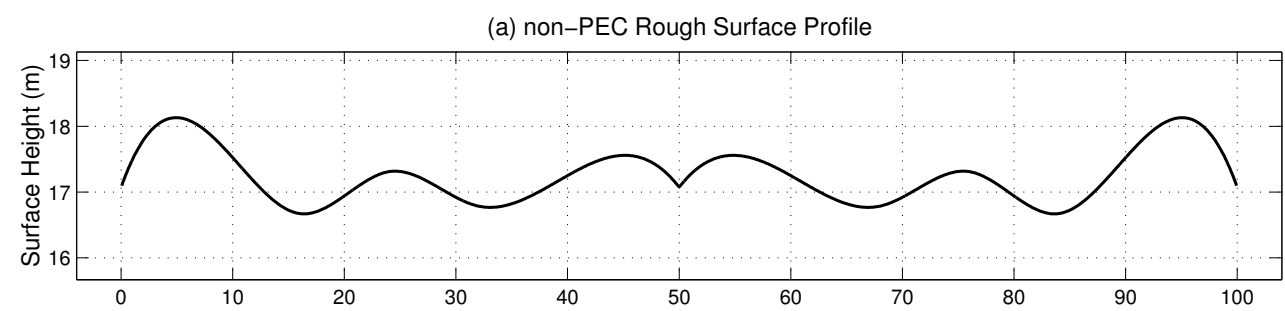

(b) TM Polarized Isotropic Radiator on non-PEC Surface (symmetric)

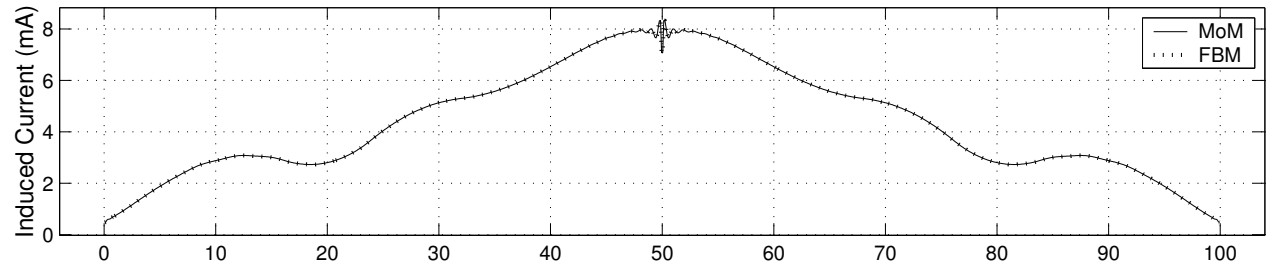

(c) TE Polarized Isotropic Radiator on non-PEC Surface (symmetric)

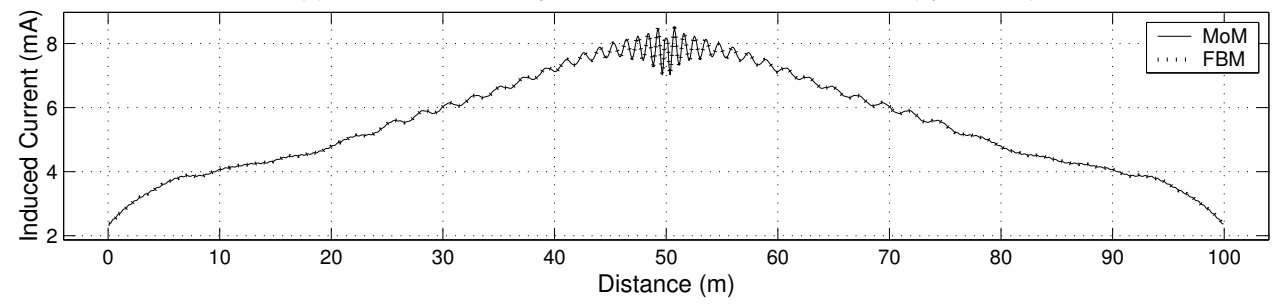

Figure 2.22: Induced current on a $100 \lambda$ rough surface

Considering a vertical polarized cut of the spherical radiation pattern of the isotropic radiator on the $x z$-plane, the impedance matrix elements are obtained 
using (2.34) and (2.35). Elements of the vector $\mathbf{V}$ are considered to be

$$
V_{n}=-H_{y}^{i n c}\left(\boldsymbol{\rho}_{n}\right)=-\frac{E_{0}}{\eta_{o}} \frac{e^{-j k d_{n}}}{d_{n}}
$$

for the consistency in the amplitude of the induced current, where

$$
d_{n}=\sqrt{\left[x_{n}-x_{1}\right]^{2}+\left[z_{n}-z_{1}\right]^{2}}
$$

and

$$
E_{0}=\sqrt{60 P_{t}}
$$

$P_{t}$ denotes the transmitted power from the isotropic radiator in $(2.66)$ and it is considered to be 25 Watts.

Figure 2.22 (b) and (c) illustrate the comparison of induced currents obtained using MoM and FBM on $100 \lambda$ non-PEC $\left(\eta_{s}=20+j 15 \Omega\right)$ rough surface profile for symmetric incident TM and TE polarized isotropic radiator cases, respectively.

The non-symmetric incidence case is considered, locating the radiator over the surface at a height above $25 \lambda$ in the beginning of the surface. Figure 2.23 (b) and (c) illustrate the comparison of induced currents obtained using MoM and FBM for non-symmetric incident TM and TE polarized isotropic radiator cases, respectively, on the same $100 \lambda$ non-PEC $\left(\eta_{s}=20+j 15 \Omega\right)$ rough surface profile.

In order to show the accuracy of the method, the residual and absolute error versus the number of iteration graphs are illustrated in Figure 2.24. In the vertical polarization case the required number of iterations to reach a desirable error level is two, while it is six in the case of horizontal polarization.

The impedance matrix in the TE case is much more diagonally dominant than that in the TM case, so that the FBM presents more accurate results within much less number of iterations in the TE case. 
(a) non-PEC Rough Surface Profile

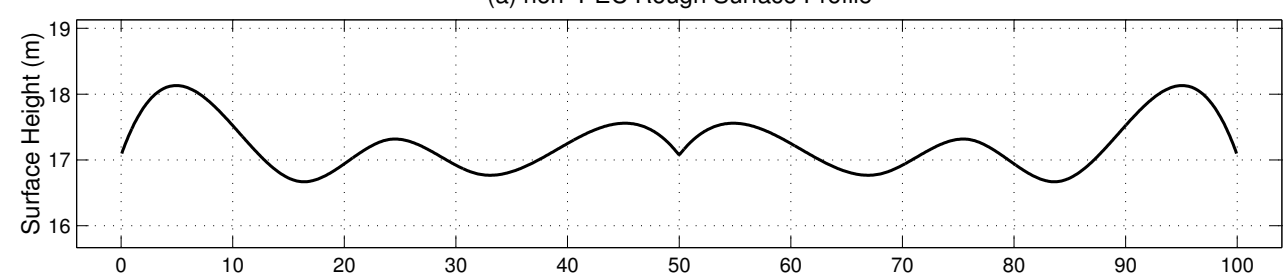

(b) TM Polarized Isotropic Radiator on non-PEC Surface (non-symmetric)

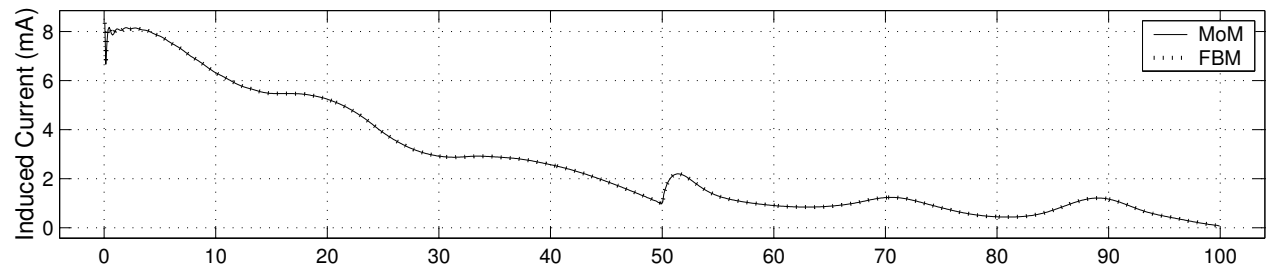

(c) TE Polarized Isotropic Radiator on non-PEC Surface (non-symmetric)

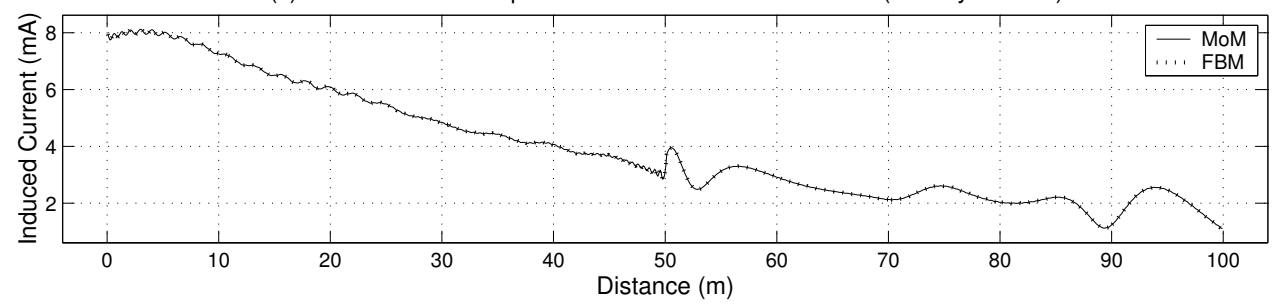

Figure 2.23: Induced current on a $100 \lambda$ rough surface
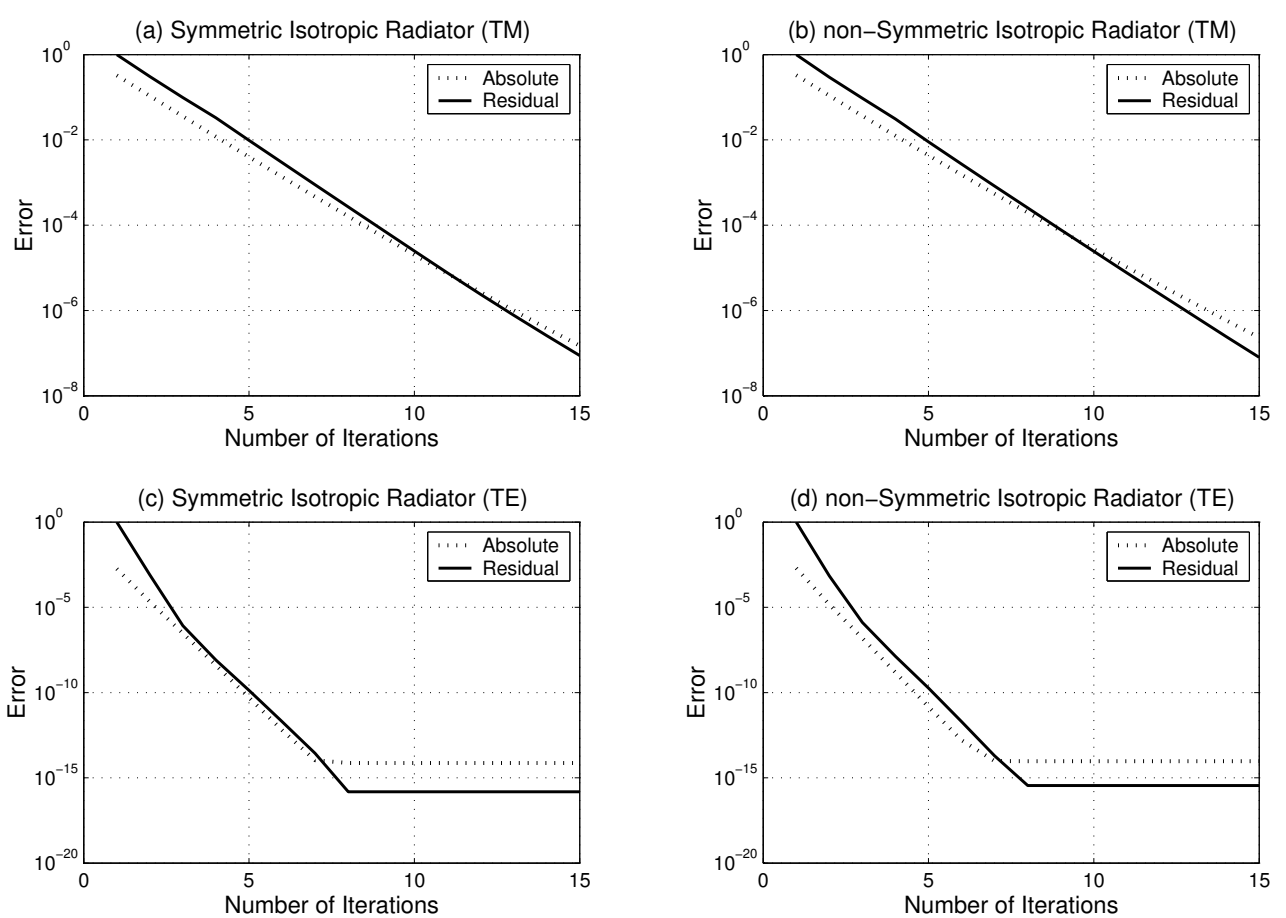

Figure 2.24: Residual and Absolute error for a $100 \lambda$ rough surface 


\section{Infinitesimal Dipole on a $200 \lambda$ rough surface}

Consider the $w=200 \lambda$ symmetric non-PEC rough surface profile illustrated in Figure 2.25 (a), which is illuminated by an infinitesimal dipole antenna located symmetrically over the profile at a height above $25 \lambda$ in the middle of the surface.

Assuming a horizontal polarization, using (2.18) and (2.19), the entries of the impedance matrix are evaluated. (2.58)-(2.60) are valid for computing the elements of $V_{n}$ for the horizontal polarization case.

In the case of vertical polarization, the impedance matrix elements are obtained using (2.34) and (2.35). Elements of the vector $\mathbf{V}$ are considered to be

$$
V_{n}=-H_{y}^{i n c}\left(\boldsymbol{\rho}_{n}\right)=-\frac{E_{0}}{\eta_{o}} \frac{e^{-j k d_{n}}}{d_{n}} \sin \theta_{n}
$$

for the consistency in the amplitude of the induced current, where

$$
\sin \theta_{n}=\frac{x_{n}-x_{1}}{d_{n}}
$$

and

$$
E_{0}=\sqrt{90 P_{t}}
$$

$d_{n}$ denotes the distance between the $n$th receiving element and the source, and it is given by $(2.65) . P_{t}$ represents the transmitted power from the infinitesimal dipole in (2.69) and it is considered to be 25 Watts.

Figure 2.25 (b) and (c) illustrate the comparison of induced currents obtained using MoM and FBM on $200 \lambda$ non-PEC $\left(\eta_{s}=20+j 20 \Omega\right)$ rough surface profile for symmetric incident TM and TE polarized infinitesimal dipole cases, respectively.

The non-symmetric incidence case is considered, locating the dipole over the profile at a height above $25 \lambda$ in the beginning of the surface. Figure 2.26 (b) and (c) illustrate the comparison of induced currents obtained using MoM and FBM for non-symmetric incident TM and TE polarized infinitesimal dipole cases, respectively, on the same $200 \lambda$ non-PEC $\left(\eta_{s}=20+j 20 \Omega\right)$ rough surface profile. 
(a) non-PEC Rough Surface Profile

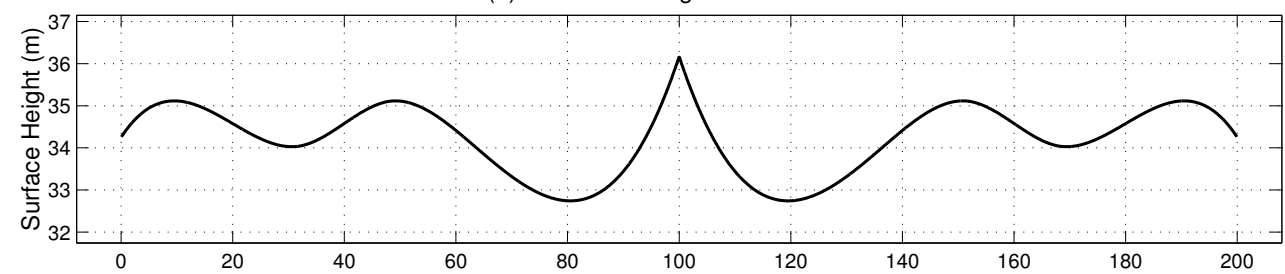

(b) TM Polarized Dipole on non-PEC Surface (Symmetric)

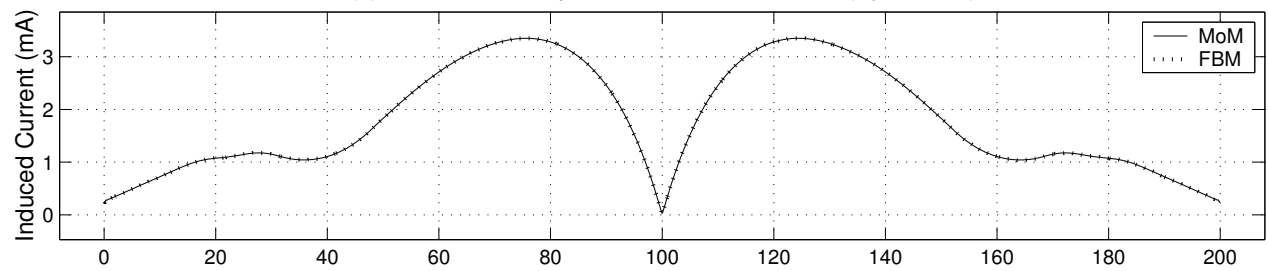

(c) TE Polarized Dipole on non-PEC Surface (Symmetric)

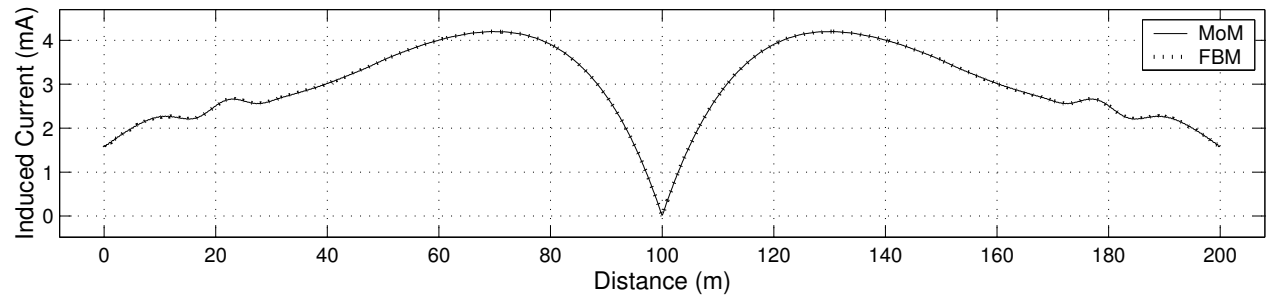

Figure 2.25: Induced current on a $200 \lambda$ rough surface

In order to show the accuracy of the method, the residual and absolute error versus the number of iteration graphs are illustrated in Figure 2.27.

In the vertical polarization case the required number of iterations to reach a desirable error level is two, while it is six in the case of horizontal polarization. The impedance matrix in the TE case is much more diagonally dominant than that in the TM case, so that the FBM presents more accurate results within much less number of iterations in the TE case.

Obtaining the MoM reference solution becomes a cumbersome problem because of the $O\left(N^{3}\right)$ computational cost, after 2000 surface unknowns. However, tests of the FBM so far shows that the FBM solution can be used as a numerically accurate reference for computing the scattering from rough surface profiles. It is observed that, after six or seven iterations for the TM polarization case and after two iterations for the TE polarization case, the values converge to an accuracy level of residual error about $10^{-3}$. 
(a) non-PEC Rough Surface Profile

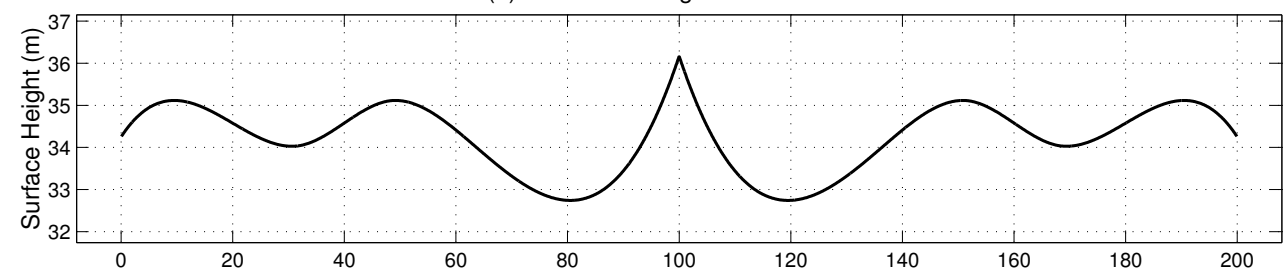

(b) TM Polarized Dipole on non-PEC Surface (non-symmetric)

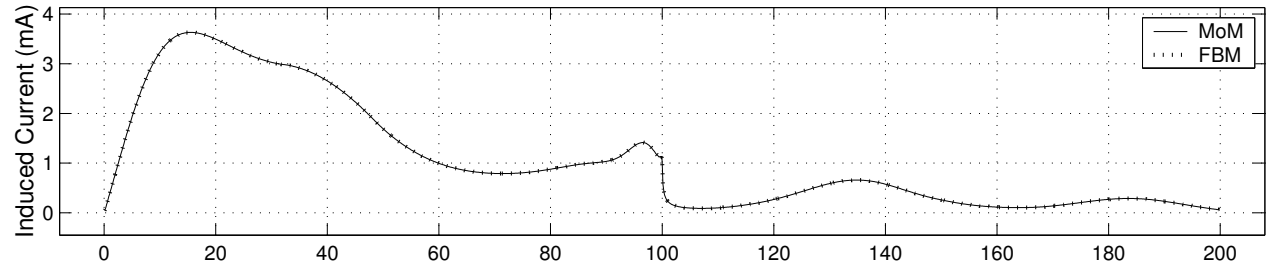

(c) TE Polarized Dipole on non-PEC Surface (non-symmetric)

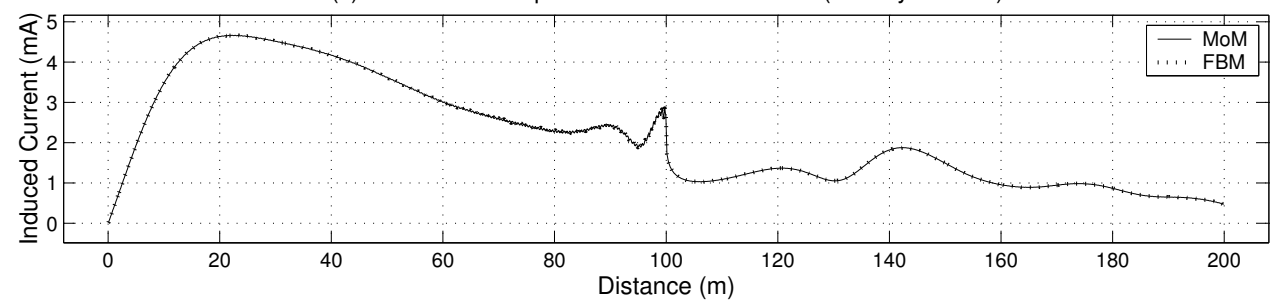

Figure 2.26: Induced current on a $200 \lambda$ rough surface
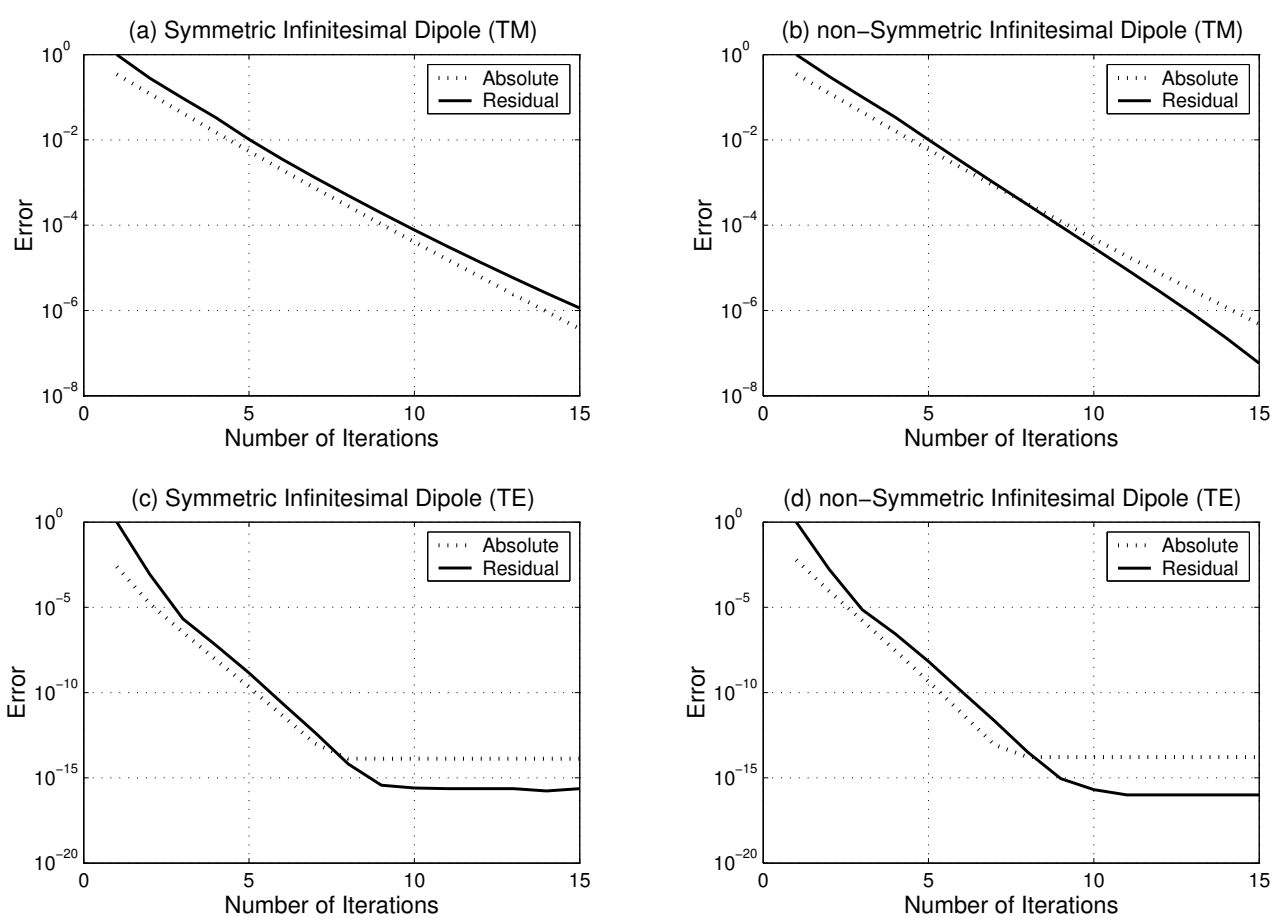

Figure 2.27: Residual and Absolute error for a 200 $\lambda$ rough surface 


\section{Plane wave incident on a $500 \lambda$ rough surface}

Consider the rough surface profile with a width of $w=500 \lambda$, excited by a finite plane wave, as illustrated in Figure 2.28 (a). The grazing incidence case is considered taking the incidence angle $\theta=\frac{\pi}{20}$. Figure 2.28 (b) and (c) illustrate the induced current obtained using FBM after six iterations for TM polarization and after two iterations for TM polarization on 500ג PEC $\left(\eta_{s}=0\right)$ rough surface profile, respectively. The effects of shadowed regions on the induced current are clearly observed for the grazing incidence case for both polarizations.

(a) PEC Rough Surface Profile

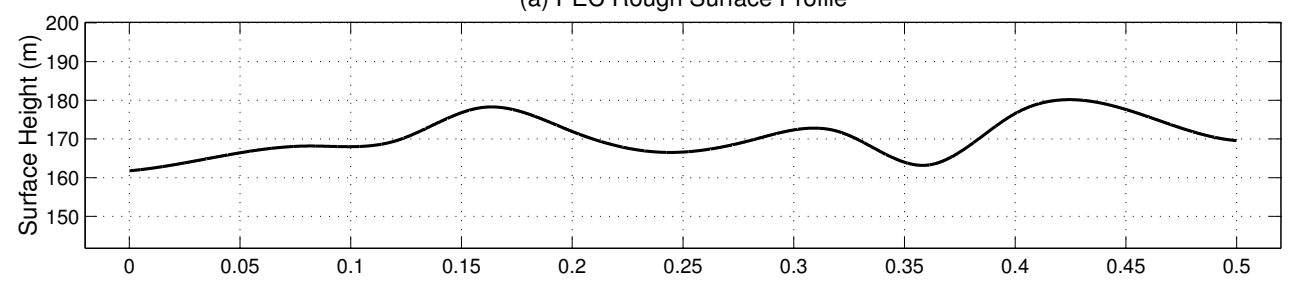

(b) TM Polarized Planewave on non-PEC Surface (Grazing)

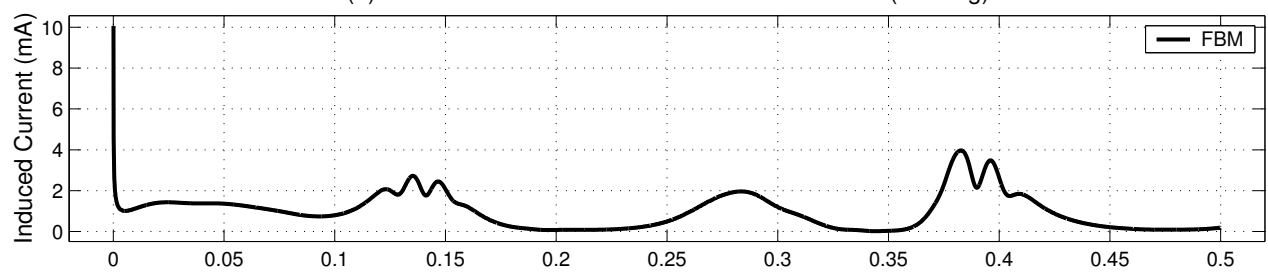

(c) TE Polarized Planewave on non-PEC Surface (Grazing)

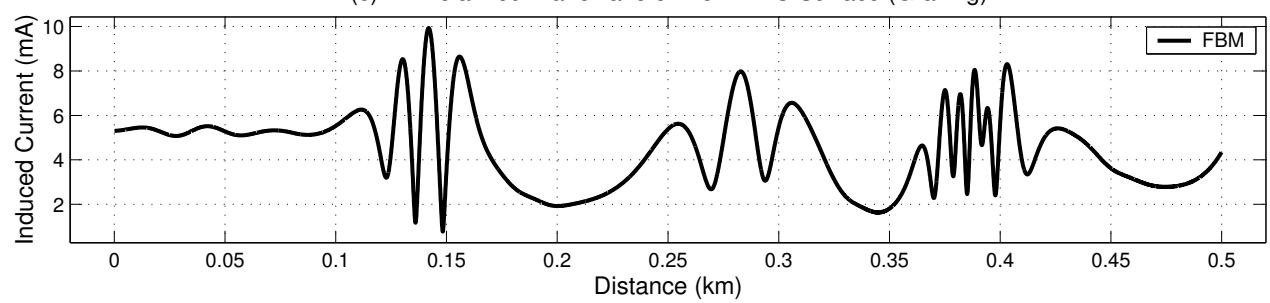

Figure 2.28: Induced current on a $500 \lambda$ rough surface

\section{Isotropic Radiator on a $1000 \lambda$ rough surface}

Figure 2.29 (a) shows a $w=1000 \lambda$ symmetric non-PEC rough surface profile, which is illuminated by an isotropic radiator located symmetrically over the surface at a height above $25 \lambda$ in the middle of the surface. Figure 2.29 (b) and (c) illustrate the induced current obtained using FBM after six iterations for 
(a) non-PEC Rough Surface Profile

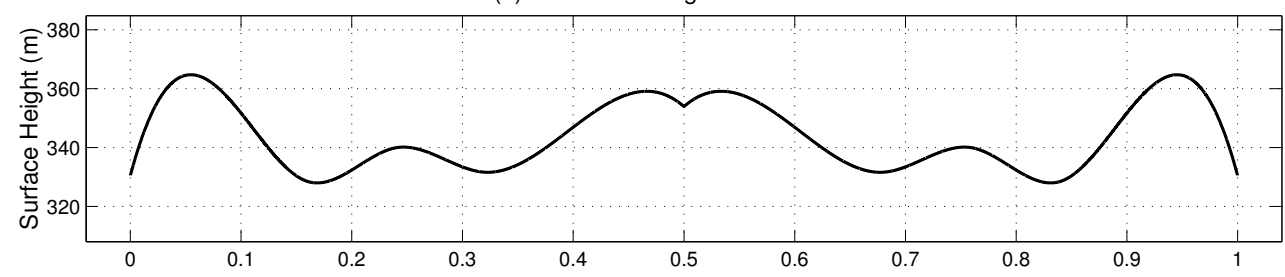

(b) TM Polarized Isotropic Radiator on non-PEC Surface (Symmetric)

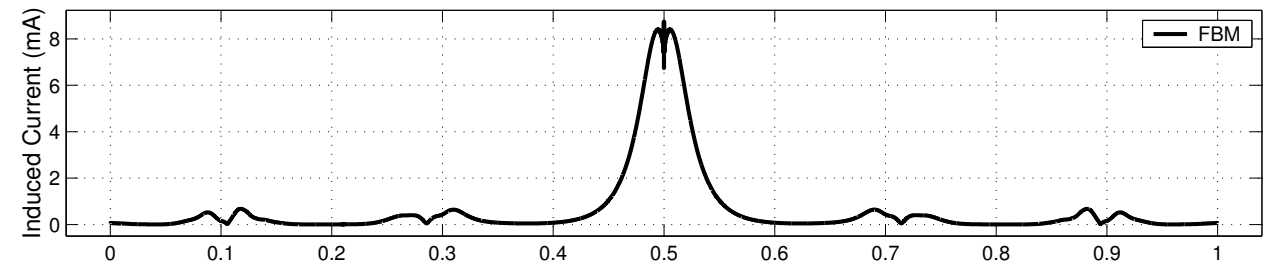

(c) TE Polarized Isotropic Radiator on non-PEC Surface (Symmetric)

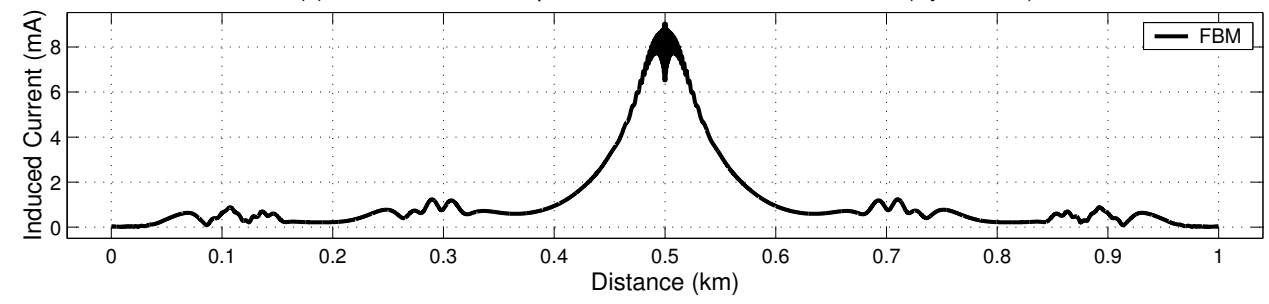

Figure 2.29: Induced current on a $1000 \lambda$ rough surface

TM polarization and after two iterations for TM polarization on $1000 \lambda$ non-PEC $\left(\eta_{s}=20+j 15 \Omega\right)$ rough surface profile, respectively.

\section{Infinitesimal Dipole on a $2000 \lambda$ rough surface}

Consider the $w=2000 \lambda$ non-PEC rough surface profile illustrated in Figure 2.30 (a), which is illuminated by an infinitesimal dipole antenna. The nonsymmetric incidence case is considered, locating the dipole over the profile at a height above $25 \lambda$ in the beginning of the surface. Taking the segment length $\Delta x=\frac{\lambda}{10}$, the surface profile can be discretized into $N=20000$ segments. Figure 2.30 (b) and (c) illustrate the induced current obtained using FBM after six iterations for TM polarization and after two iterations for TE polarization on $2000 \lambda$ non-PEC $\left(\eta_{s}=20+j 20 \Omega\right)$ rough surface profile, respectively.

Investigations over rough surface profiles show that the FBM obtains the numerically accurate solutions within very few iterations on rough surfaces for 

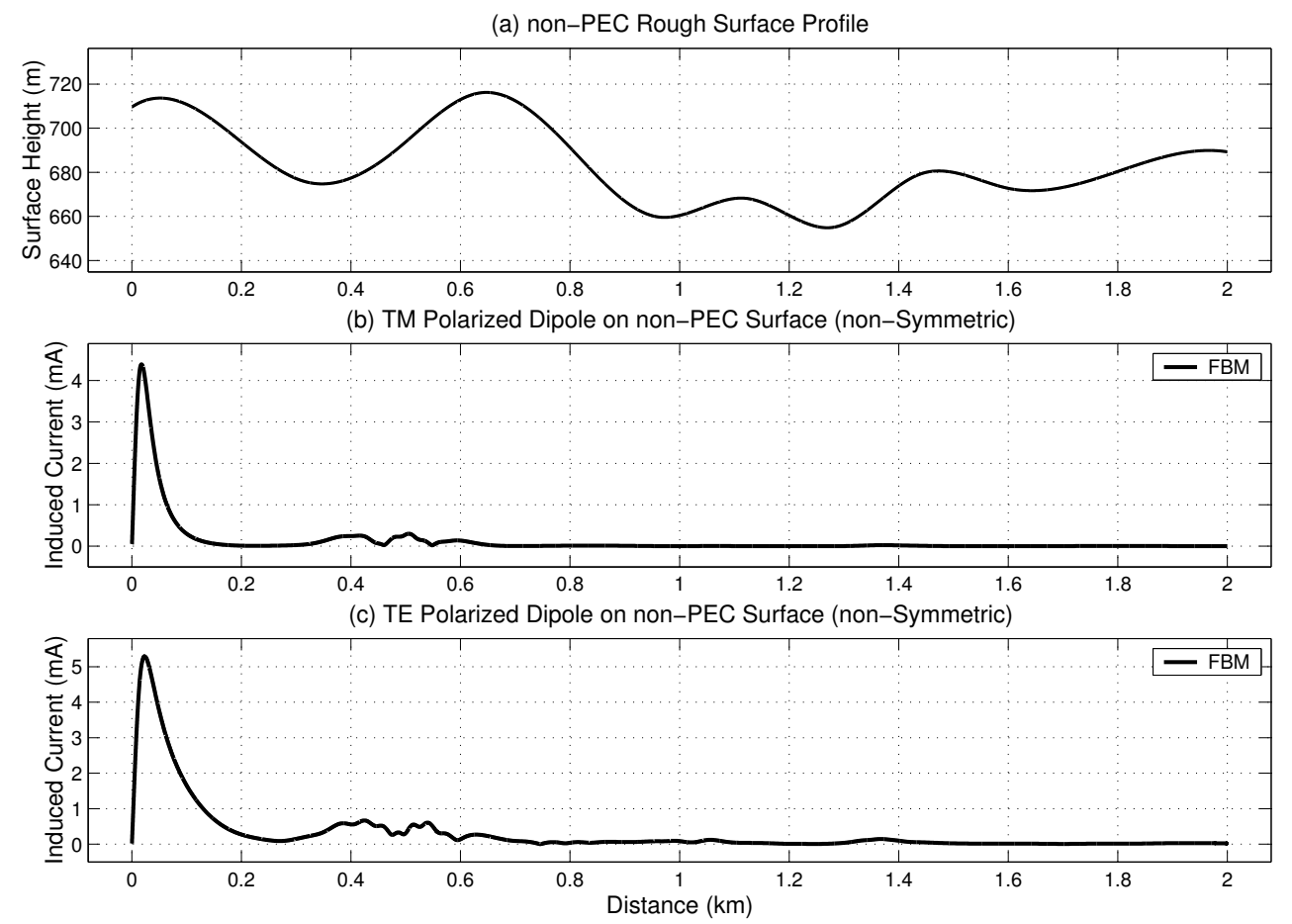

Figure 2.30: Induced current on a $2000 \lambda$ rough surface

both horizontal and vertical polarization cases. In order to compare the computational cost of the method with MoM, the computation time versus number of iterations examinations are illustrated in Figure 2.31 and in Table 2.2 for both of the reference methods. The FBM reaches a desirable level of error after six iterations in the TM case and after two iterations in the TE case, when the values converge to an accuracy level of residual error about $10^{-3}$. Computational cost of the matrix filling and direct inversion (LU) for the MoM are illustrated, too.

The cpu-time for the TE-FBM is nearly the same as the matrix filling time. Note that, after 2000 unknowns, cpu-time values for the MoM are obtained using a spline extrapolation of those for less than 2000 .

It is obvious that, the FBM presents a computational cost of $O\left(N^{2}\right)$ while the direct solution of the MoM requires $O\left(N^{3}\right)$ cpu-time, where $N$ is the total number of surface unknowns. 


\begin{tabular}{|c|c|c|c|c|}
\hline$N$ & Matrix Fill (s) & LU Inversion (s) & TM-FBM (s) & TE-FBM (s) \\
\hline 500 & 1.5 & 59.5 & 9 & 1.5 \\
1000 & 6.5 & 542 & 35 & 5.9 \\
2000 & 25.5 & 5891.5 & 142 & 23.6 \\
5000 & 154.5 & NA & 88.7 & 172 \\
10000 & 609.5 & NA & 377.5 & 681 \\
20000 & 2419.5 & NA & 15805 & 2811 \\
\hline
\end{tabular}

Table 2.2: Computational Cost

The investigations and comparisons demonstrate that, the FBM can be used as a numerically accurate reference solution instead of MoM over rough surface profiles in both horizontal and vertical polarization cases, because of its very accurate results within very few iterations, causing a computational time of $O\left(N^{2}\right)$ and a memory requirement of $O(N)$.

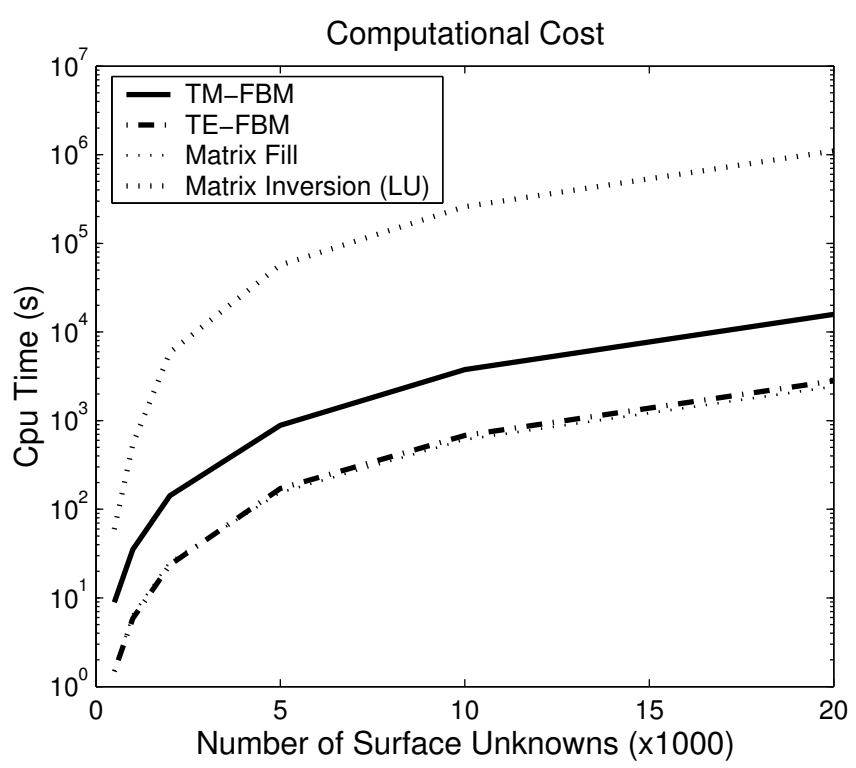

Figure 2.31: Computational Cost

\section{Computation of the Scattered Field}

Once the current distribution over the rough surface profile have been computed by the Forward-Backward iterative process, the next step is to compute the scattered field. If the region of interest corresponds to a small portion of the surface, the numerical evaluation the total field will involve a reduced number 
of operations, but if these regions are extended to the complete terrain profile and the field strength is computed in a dense set of points, similar to the MoM discretization, the cpu-cost will increase up to $O\left(N^{2}\right)$, since the scattered field is expressed as,

$$
\begin{aligned}
E_{y}^{s c a t}\left(\boldsymbol{\rho}_{n}\right) & =-j \omega A_{y}-\frac{1}{\epsilon}\left\{\frac{\partial F_{x}}{\partial z}-\frac{\partial F_{z}}{\partial x}\right\} \\
& \cong-\frac{\omega \mu}{4} \sum_{m=1}^{N} I_{m} \Delta x_{m} H_{0}^{(2)}\left(k\left|\boldsymbol{\rho}_{n}-\boldsymbol{\rho}_{m}\right|\right) \\
& -j \frac{k}{4} \sum_{m=1}^{N} I_{m} \Delta x_{m} \eta_{m} H_{1}^{(2)}\left(k\left|\boldsymbol{\rho}_{n}-\boldsymbol{\rho}_{m}\right|\right) \hat{n}_{m} \cdot \hat{\rho}_{n m}
\end{aligned}
$$

and

$$
\begin{aligned}
H_{y}^{\text {scat }}\left(\boldsymbol{\rho}_{n}\right) & =-j \omega F_{y}+\frac{1}{\mu}\left\{\frac{\partial A_{x}}{\partial z}-\frac{\partial A_{z}}{\partial x}\right\} \\
& \cong \frac{\omega \epsilon}{4} \sum_{m=1}^{N} I_{m} \Delta x_{m} \eta_{m} H_{0}^{(2)}\left(k\left|\boldsymbol{\rho}_{n}-\boldsymbol{\rho}_{m}\right|\right) \\
& +j \frac{k}{4} \sum_{m=1}^{N} I_{m} \Delta x_{m} H_{1}^{(2)}\left(k\left|\boldsymbol{\rho}_{n}-\boldsymbol{\rho}_{m}\right|\right) \hat{n}_{m} \cdot \hat{\rho}_{n m}
\end{aligned}
$$

for TM and TE polarization cases respectively. Here $I_{m}$ denotes the computed induced current on the source point $\boldsymbol{\rho}_{m}$, and $\boldsymbol{\rho}_{n}$ denotes the observation point on where the scattered field will be obtained.

Consider the $w=2000 \lambda$ non-PEC rough surface profile illustrated in Figure 2.32 (a), which is illuminated by an infinitesimal dipole antenna. The nonsymmetric incidence case is considered, locating the dipole over the profile at a height above $25 \lambda$ in the beginning of the surface. Figure 2.30 (b) and (c) illustrate the induced current obtained using FBM after six iterations for TM polarization and after two iterations for TE polarization on $2000 \lambda$ non-PEC $\left(\eta_{s}=20+j 20 \Omega\right)$ rough surface profile, respectively.

2.32 (a) also illustrates a copy of the surface profile, where the scattered field is going to be computed, sited $h=1.8 \lambda$ above the original terrain. Figure 2.32 (b) and (c) illustrate the total field $E_{y}^{t o t}=E_{y}^{i n c}+E_{y}^{s c a t}$ obtained using (3.37) for TM 
(a) non-PEC Rough Surface Profile

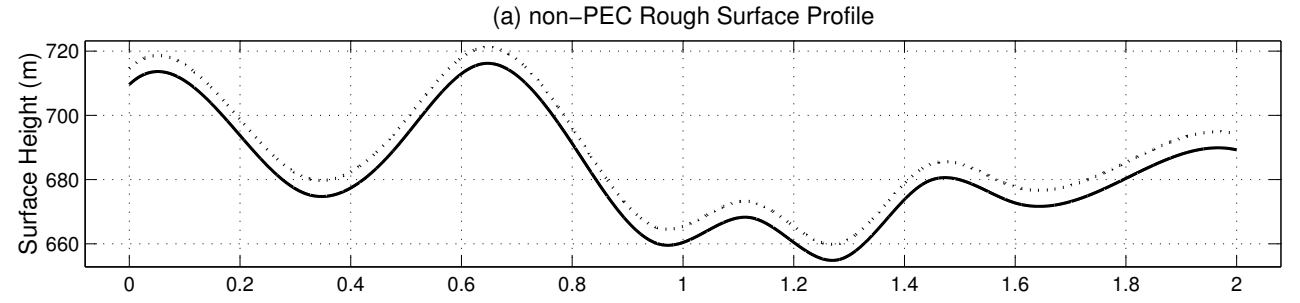

(b) TM Polarized Dipole on non-PEC Surface (non-Symmetric)

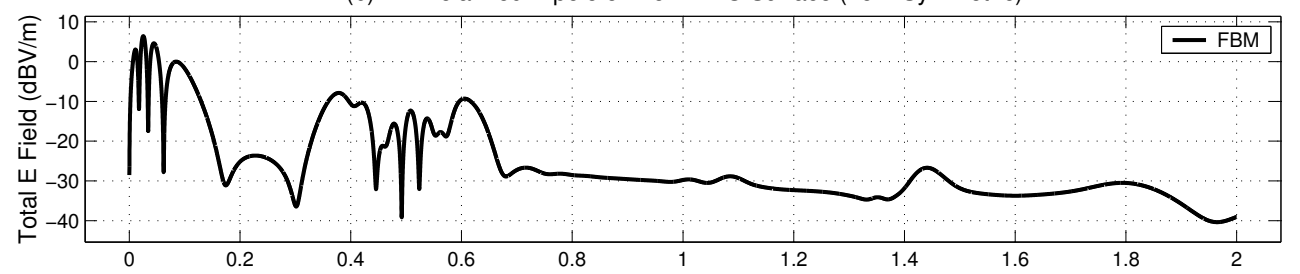

(c) TE Polarized Dipole on non-PEC Surface (non-Symmetric)

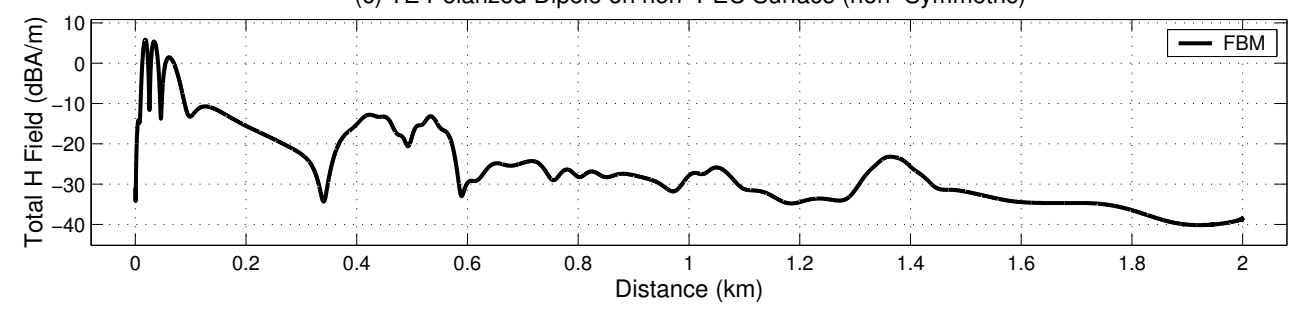

Figure 2.32: Total Field at $h=1.8 \mathrm{~m}$ on a $2000 \lambda$ rough surface

polarization and $H_{y}^{\text {tot }}=H_{y}^{i n c}+H_{y}^{\text {scat }}$ obtained using (3.38) for TE polarization on $2000 \lambda$ non-PEC $\left(\eta_{s}=20+j 20 \Omega\right)$ rough surface profile, respectively. The effects of shadowed regions on the total field are clearly observed for both polarizations.

\section{The Limitations of the FBM}

The FBM algorithm is a stationary iterative process and, in fact, mathematically equivalent to the well-known symmetric successive over relaxation - SSOR iteration [25]. This method is very good at obtaining accurate results, when the matrix in the linear equation system is diagonally dominant. Changing the order of current elements disturbs the diagonally dominant nature, which then strongly affects the convergence of the method. The algorithm may become unstable for re-entrant surfaces where current elements are not numbered sequentially as a function of increasing $x$ coordinate. 
(a) PEC Rough Surface Profile

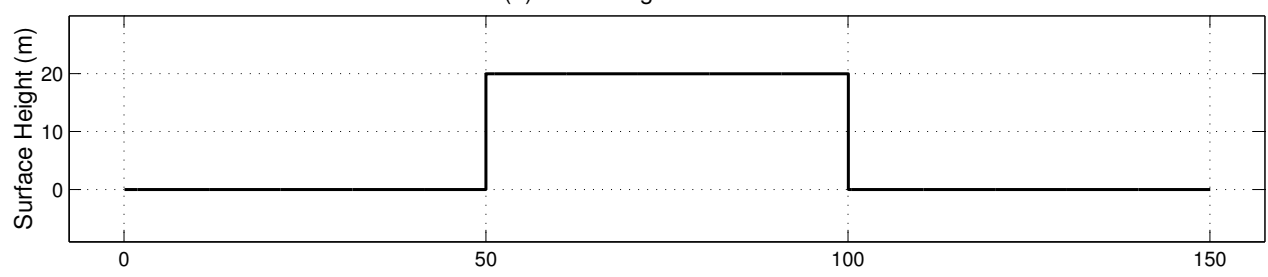

(b) TM Polarized Planewave on PEC Surface (Grazing)

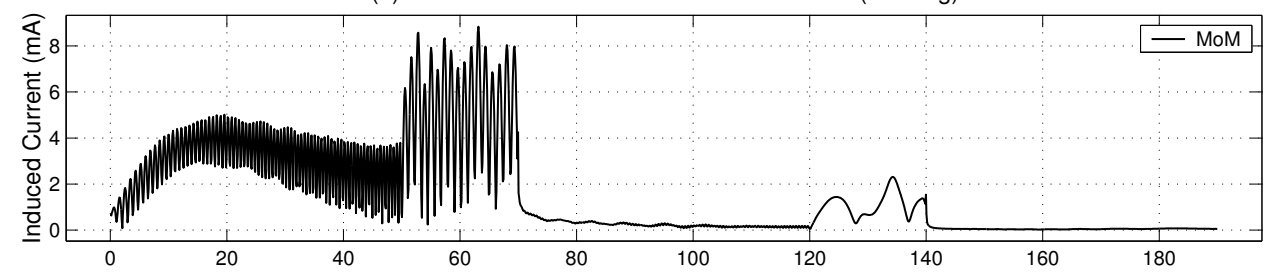

(c) TM Polarized Planewave on PEC Surface (Grazing)

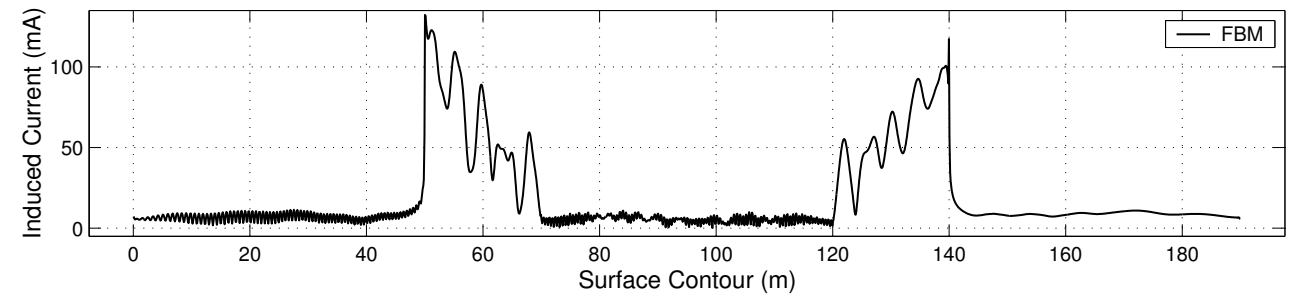

Figure 2.33: Induced current on a $150 \lambda$ rough surface

Consider the $w=150 \lambda$ PEC surface profile illustrated in Figure 2.33 (a), which is illuminated by a grazing incident plane wave. Taking the segment length $\Delta x=\frac{\lambda}{10}$, the surface contour can be discretized into $N=1900$ segments. Figure 2.33 (b) illustrates the induced current obtained using the MoM and (c) illustrates the one obtained from the FBM for TM polarization case on $150 \lambda$ PEC $\left(\eta_{s}=0\right)$ rough surface profile. It is observed that even after 15 iterations, the FBM result could not reach an accurate value of induced current. The residual and absolute error graphs are plotted in Figure 2.34.

The induced current obtained using the FBM for the TE polarization could not even converge to a value and gave $N a N$ current distribution. This limitation of the FBM has been overcome by the Generalized Forward-Backward Method (GFBM), which is a hybrid method based on a combination of the conventional FBM with MoM [26]. 

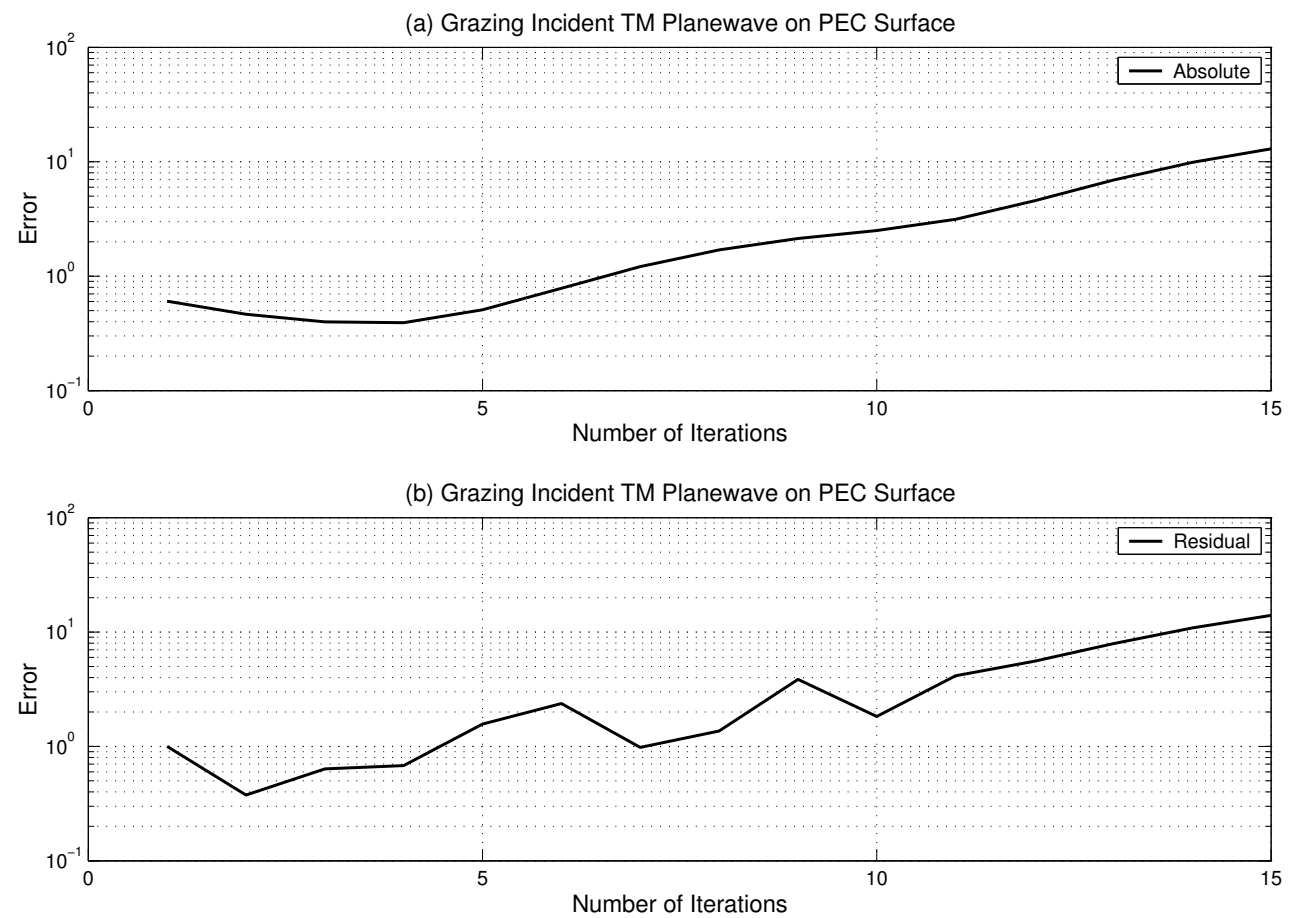

Figure 2.34: Residual and absolute error

Non-stationary iterative techniques, such as Conjugate Gradient Method, are not affected by the order of the surface interactions in the impedance matrix. Hence, they can handle any kind of such re-entrant surfaces. However, their ability to converge is not as fast as that of stationary algorithms. The number of iterations to approach a desired level of error often reaches to hundreds without preconditioning techniques. In order to use a preconditioner, the entries of the matrix should be evaluated and stored, so that the use of non-stationary algorithms are not as computationally efficient as the use of stationary ones. 


\section{Chapter 3}

\section{SPECTRAL ACCELERATION OF THE FBM}

The Forward-Backward Method has been shown to provide a more rapid convergence than a standard non-stationary iterative algorithm in many cases. In this method, the current due to the forward propagating field contribution is first found for every receiving element on the surface and then employed to recover the backward propagating field contribution in an iterative procedure. This procedure presents very accurate results within very few iterations. However, the FBM obtains an operational count of $O\left(N^{2}\right)$ in the matrix-vector multiplication, and in order to avoid $O\left(N^{2}\right)$ memory storage, a time-consuming computation of impedance matrix elements needs to be repeated on every iteration. This computational cost prohibits the application of the FBM to large-scale scattering problems.

The Spectral Acceleration algorithm was proposed to overcome the computational limitation of the FBM over one-dimensional slightly rough PEC surfaces by Chou and Johnson in [7]. This algorithm accelerates the matrix-vector multiplications in the FBM and divides contributions between points into strong and 
weak regions. The spectral acceleration is based on a spectral representation of the two-dimensional Green's function and appropriate contour deformation, producing a fast multipole-like formulation in which contributions from large number of points to a single point are evaluated simultaneously. However, unlike traditional multipole methods, only one large group of points is considered for the calculation of weak region contributions, and this results a more efficient computation.

In order to further improve the efficiency of the spectral expansion, the angular spectral integration path is deformed into the complex angular plane to obtain a smaller domain of integration with less rapid oscillation along the integration path. The multiplication is performed in a forward sweep followed by a backward sweep, with the weak region continuously increasing in size as the multiplication proceeds in one direction. Because of the use of forward and backward sweeps, the spectral acceleration approach is well suited for the FBM, but can also be used in any standard iterative method.

This technique reduces the computational cost and memory requirements to $O(N)$, so that the Spectrally Accelerated Forward-Backward Method (FBSA) can be applied over very large one-dimensional quasi-planar surfaces. Chou and Johnson extended the Spectrally Accelerated Forward-Backward Method (FBSA) formulation to treat impedance surfaces [27]. A multilevel version of the spectral acceleration algorithm was introduced in [31]. It should be noted that these original implementations of the spectral acceleration algorithm were developed to analyze quasi-planar (slightly rough) surfaces such as ocean-like surfaces, and becomes not suitable for very undulating geometries. López et al. modified the spectral acceleration algorithm in order to implement FBSA to very undulating rough surfaces such as terrain profiles [21]. The spectral acceleration algorithm was adapted to the GFBM in [32]. Moss et al. calculated the scattering from layered rough surfaces using the FBSA in [40]. The FBSA was 
implemented to two-dimensional PEC and impedance surfaces, respectively in [41] and [42].

This chapter is devoted to the discussion of the Spectral Acceleration of the Forward-Backward Method. The spectral acceleration for quasi-planar rough surfaces and its adaptation over very undulating terrain profiles are described in Sections 3.1 and 3.2, respectively. Section 3.3 presents numerical results and limitations of the FBSA over several sample rough surfaces.

\subsection{The FBSA for Quasi-Planar Rough Sur- faces}

The iterative FBM procedure requires the repeated computation of the matrixvector products denoting the forward and backward radiations of the source elements preceding and following the $n$th receiving element, respectively. It is obvious that this computation requires an $O\left(N^{2}\right)$ operational count. The acceleration algorithm for computing forward and backward radiations in $O(N)$ begins with selection of a neighborhood distance $L_{s}$ from the receiving element as illustrated in Figure 3.1. Thus, the radiating elements over a given receiving element are divided into two groups: strong interactions group $G_{s}$ and weak interactions group $G_{w}$. So, for a given neighborhood distance $L_{s}$, the strong group $G_{s}$ includes the $N_{s}$ nearest elements to the receiving element while the rest radiating elements are included in the weak group $G_{w}$. With this decomposition, the fields radiated over the receiving element can be expressed as the sum of the weak and strong group contributions.

The spectral acceleration algorithm is based on the fast computation of the far elements radiation by using a spectral representation of the two-dimensional Green's function. Thus, the radiation of the strong interaction group is computed 
through the FBM matrix-vector products in an operational count of $O\left(N_{s}^{2}\right)$, but to the weak interactions, the spectral acceleration is applied for the computation of weak interactions producing a computational cost of $O(N)$. Since the number of surface unknowns in the strong group is extremely less than that in the weak region, the total operational count becomes $O(N)$.

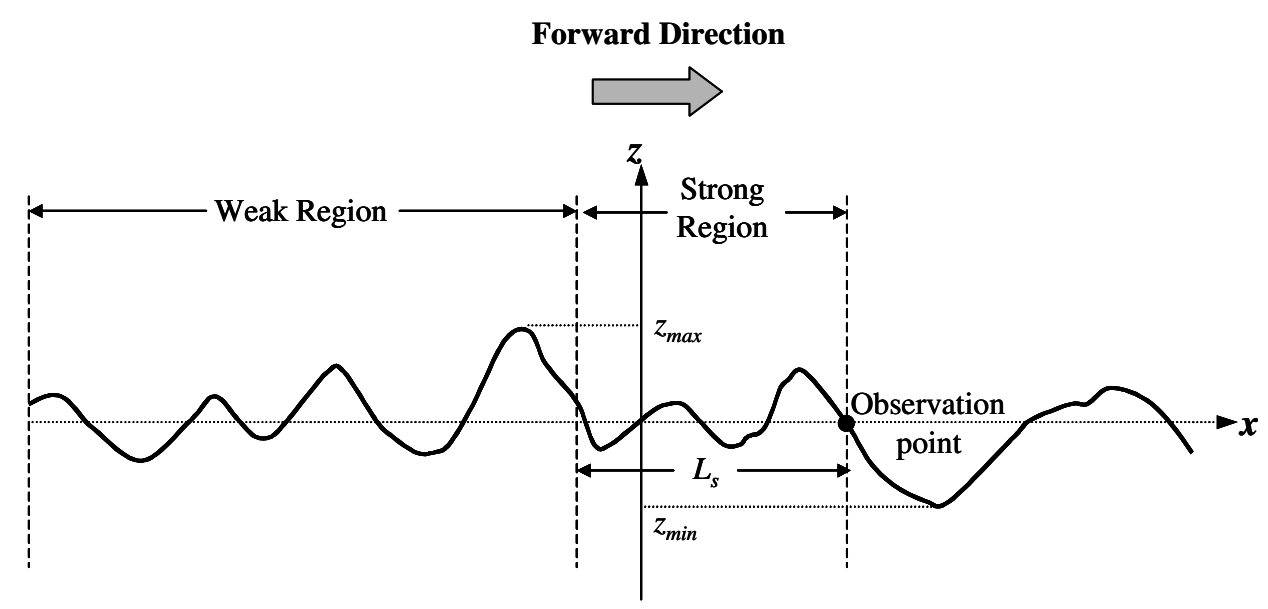

Figure 3.1: 1-D finite rough surface profile.

Since the FBM formulation is derived for both horizontal and vertical polarizations, the spectral acceleration will be described in Sections 3.1.1 and 3.1.2, respectively, in the same manner. Then, the appropriate deformation of the integration contour and the integration parameters will be discussed.

\subsubsection{Spectral Acceleration of the FBM for Horizontal Polarization}

The FBM procedure of the electric field integral equation for horizontal polarization requires repeated computation of the matrix-vector products $\overline{\mathbf{Z}}^{f}$. I and $\overline{\mathbf{Z}}^{b} \cdot \mathbf{I}$ as

$$
E_{f}\left(\boldsymbol{\rho}_{n}\right)=\sum_{m=1}^{n-1} I_{m} Z_{n m}
$$




$$
E_{b}\left(\boldsymbol{\rho}_{n}\right)=\sum_{m=n+1}^{N} I_{m} Z_{n m}
$$

where $N$ is the number of surface unknowns. (A.1) and (A.2) denotes the forward and backward radiations of the source elements in front and at the rear of the $n$th receiving element, respectively. Selecting a neighborhood distance $L_{s}$ for a receiving element, within which interactions are classified as strong and outside of which interactions are classified as weak, the acceleration algorithm for computing (A.1) and (A.2) in $O(N)$ begins. As a result (A.1) can be rewritten as

$$
\begin{gathered}
E_{f}\left(\boldsymbol{\rho}_{n}\right)=E_{s}\left(\boldsymbol{\rho}_{n}\right)+E_{w}\left(\boldsymbol{\rho}_{n}\right) \\
=\sum_{m=n-N_{s}}^{n-1} I_{m} Z_{n m}+\sum_{m=1}^{n-N_{s}-1} I_{m} Z_{n m},
\end{gathered}
$$

where $N_{s}=L_{s} / \Delta x$ denotes the number of elements that have strong interactions with the $n$th element. The first term in (A.3) denoted by $E_{s}$ represents the strong region contributions and the second term denoted by $E_{w}$ represents the weak region contributions. The off-diagonal entries of the impedance matrix for horizontal polarization were derived using the EFIE in the previous chapter for non-PEC surfaces as

$$
Z_{n m}=-j \omega \mu G\left(\boldsymbol{\rho}_{n}, \boldsymbol{\rho}_{m}\right) \Delta x_{m}+\eta_{m} \Delta x_{m} \frac{\partial}{\partial n_{m}} G\left(\boldsymbol{\rho}_{n}, \boldsymbol{\rho}_{m}\right)
$$

For simplicity only the application of the spectral acceleration to the forward propagation will be described. The backward propagation equation, (A.2), can be treated in the same fashion and is given in Appendix A.

The strong region contributions $E_{s}$ are found in the conventional FBM manner using the exact matrix elements, while the rapid computation of the weak region contributions $E_{w}$ is performed by employing the spectral representation of the two-dimensional Green's function and its partial derivative. The spectral representation of the Green's function is expressed as:

$$
G\left(\boldsymbol{\rho}_{n}, \boldsymbol{\rho}_{m}\right)=\frac{-j}{4 \pi} \int_{C_{\phi}} e^{-j k\left[\left(x_{n}-x_{m}\right) \cos \phi+\left(z_{n}-z_{m}\right) \sin \phi\right]} d \phi
$$


where $C_{\phi}$ is the contour of integration in the complex $\phi$ space shown in Figure 3.2. On the other hand, the spectral representation of the partial derivative of the Green's function with respect to the normal vector on source point can be expressed as:

$$
\begin{aligned}
\frac{\partial}{\partial n_{m}} G\left(\boldsymbol{\rho}_{n}, \boldsymbol{\rho}_{m}\right)= & \frac{k}{4 \pi} \int_{C_{\phi}}\left[\cos \theta_{m} \cos \phi+\sin \theta_{m} \sin \phi\right] \\
& \cdot e^{-j k\left[\left(x_{n}-x_{m}\right) \cos \phi+\left(z_{n}-z_{m}\right) \sin \phi\right]} d \phi
\end{aligned}
$$

where $\theta_{m}$ is the angle between the unit normal vector to the surface at the source point, $\hat{n}_{m}$, and the unit vector in the $x$ direction, $\hat{x}$.

Substituting (A.5) and (A.6) into (A.3) and interchanging the integration and summation, yields to:

$$
E_{w}\left(\boldsymbol{\rho}_{n}\right)=-\frac{\omega \mu}{4 \pi} \int_{C_{\phi}} F_{n}(\phi) d \phi
$$

where

$$
\begin{aligned}
F_{n}(\phi)= & \sum_{m \in G_{w}} I_{m} \Delta x_{m}\left\{1-\frac{\eta_{m}}{\eta_{o}}\left[\cos \theta_{m} \cos \phi+\sin \theta_{m} \sin \phi\right]\right\} \\
& \cdot e^{-j k\left[\left(x_{n}-x_{m}\right) \cos \phi+\left(z_{n}-z_{m}\right) \sin \phi\right]}
\end{aligned}
$$

showing that weak element contributions at point $\boldsymbol{\rho}_{n}$ can be obtained through a spectral domain integral of the weak element complex far-field pattern or plane wave spectrum, $F_{n}(\phi)$.

It is at this point where the great reduction in computational cost is obtained because $F_{n}(\phi)$ can be easily updated using a recursive procedure. In general, $F_{n}(\phi)$ can be expressed as a function of $F_{n-1}(\phi)$ as follows [7]:

$$
\begin{aligned}
F_{n}(\phi)= & F_{n-1}(\phi) e^{-j k\left[\left(x_{n}-x_{n-1}\right) \cos \phi+\left(z_{n}-z_{n-1}\right) \sin \phi\right]} \\
& +I_{n s} \Delta x_{n s}\left\{1-\frac{\eta_{n s}}{\eta_{o}}\left[\cos \theta_{n s} \cos \phi+\sin \theta_{n s} \sin \phi\right]\right\} \\
& \cdot e^{-j k\left[\left(x_{n}-x_{n s}\right) \cos \phi+\left(z_{n}-z_{n s}\right) \sin \phi\right]}
\end{aligned}
$$

with $F_{n}(\phi)=0$ for $n \leq N_{s}+1$ in the forward sweep. In (A.9), $n s=n-$ $N_{s}-1$ is the new source point introduced in the weak group as the iterative 


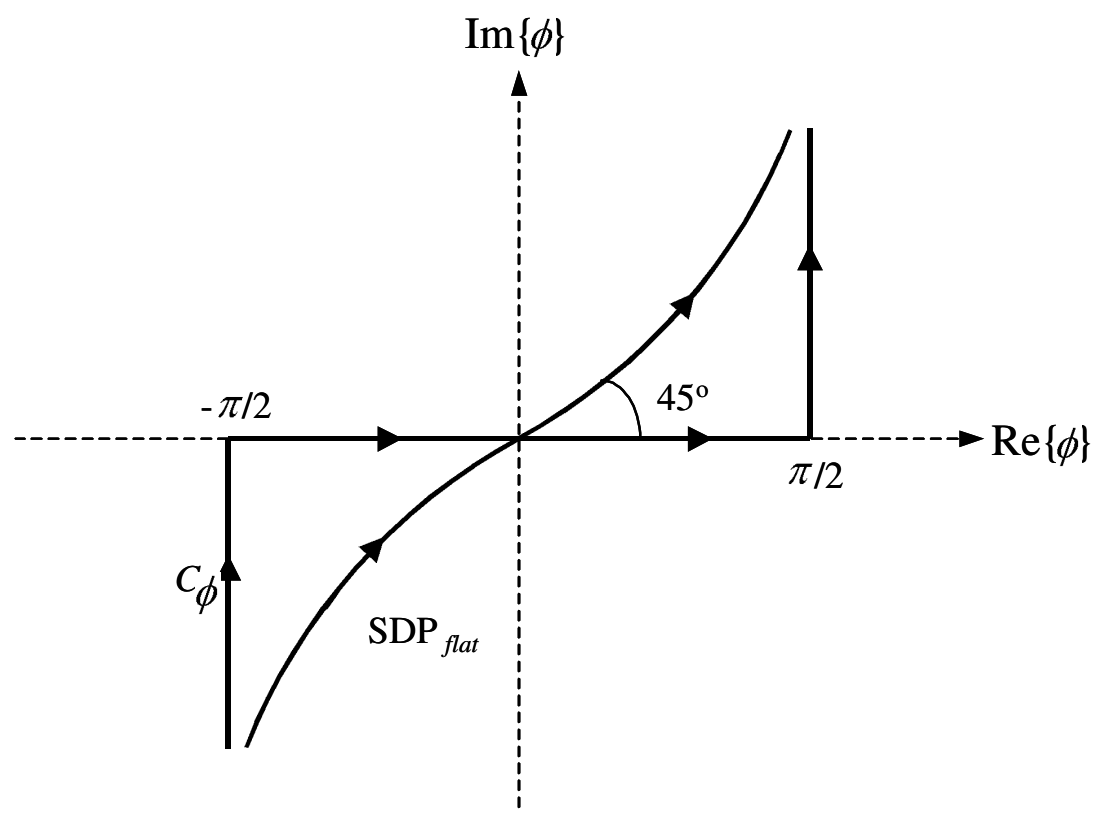

Figure 3.2: Integration path in complex space.

procedure sweeps the surface in the forward direction. Namely, (A.9) shows that $F_{n}(\phi)$ continuously updates as each new source element enters the weak interaction group in the forward sweep, and hence, the complex far field pattern at the receiving element is expressed in terms of that at the previous one. This procedure produces an $O(N)$ operational cost. An analogous procedure is used for the backward propagation.

\subsubsection{Spectral Acceleration of the FBM for Vertical Po- larization}

Similar to the horizontal polarization case, when using the magnetic field integral equation in the vertical polarization case, the radiating elements are also divided into two groups: the strong interactions region $G_{s}$, and the weak interactions region $G_{w}$. And their contribution to the forward propagating magnetic field $H_{f}$ 
can be expressed as follows:

$$
\begin{gathered}
H_{f}\left(\boldsymbol{\rho}_{n}\right)=H_{s}\left(\boldsymbol{\rho}_{n}\right)+H_{w}\left(\boldsymbol{\rho}_{n}\right) \\
=\sum_{m=n-N_{s}}^{n-1} I_{m} Z_{n m}+\sum_{m=1}^{n-N_{s}-1} I_{m} Z_{n m},
\end{gathered}
$$

where $N_{s}=L_{s} / \Delta x$ denotes the number of elements that have strong interactions with the $n$th element. The first term in (A.10) denoted by $H_{s}$ represents the strong region contributions and the second term denoted by $H_{w}$ represents the weak region contributions. The off-diagonal entries of the impedance matrix for vertical polarization were derived using the MFIE in the previous chapter for non-PEC surfaces as

$$
Z_{n m}=j \omega \epsilon \eta_{m} \Delta x_{m} G\left(\boldsymbol{\rho}_{n}, \boldsymbol{\rho}_{m}\right)-\Delta x_{m} \frac{\partial}{\partial n_{m}} G\left(\boldsymbol{\rho}_{n}, \boldsymbol{\rho}_{m}\right)
$$

For simplicity only the application of the spectral acceleration to the forward propagation will be described. The backward propagation of the magnetic field, $H_{b}$, can be treated in the same fashion and is given in Appendix A.

The strong region contributions $H_{s}$ are found in the conventional FBM manner using the exact matrix elements, while the rapid computation of the weak region contributions $H_{w}$ is performed by employing the spectral representation of the two-dimensional Green's function and its partial derivative.

Substituting the spectral representation of the two-dimensional Green's function, (A.5), and its partial derivative with respect to the unit normal vector to the surface on the source location, (A.6), into (A.10) and interchanging the integration and summation, yields to:

$$
H_{w}\left(\boldsymbol{\rho}_{n}\right)=-\frac{k}{4 \pi} \int_{C_{\phi}} F_{n}(\phi) d \phi
$$

where

$$
\begin{aligned}
F_{n}(\phi)= & \sum_{m \in G_{w}} I_{m} \Delta x_{m}\left\{\cos \theta_{m} \cos \phi+\sin \theta_{m} \sin \phi-\frac{\eta_{m}}{\eta_{o}}\right\} \\
& \cdot e^{-j k\left[\left(x_{n}-x_{m}\right) \cos \phi+\left(z_{n}-z_{m}\right) \sin \phi\right]}
\end{aligned}
$$


showing that weak element contributions at point $\boldsymbol{\rho}_{n}$ can be obtained through a spectral domain integral of the weak element complex far-field pattern or plane wave spectrum, $F_{n}(\phi)$.

$F_{n}(\phi)$ can be easily updated using a recursive procedure. In general, $F_{n}(\phi)$ can be expressed as a function of $F_{n-1}(\phi)$ as follows [32].

$$
\begin{aligned}
F_{n}(\phi)= & F_{n-1}(\phi) e^{-j k\left[\left(x_{n}-x_{n-1}\right) \cos \phi+\left(z_{n}-z_{n-1}\right) \sin \phi\right]} \\
& +I_{n s} \Delta x_{n s}\left\{\cos \theta_{n s} \cos \phi+\sin \theta_{n s} \sin \phi-\frac{\eta_{n s}}{\eta_{o}}\right\} \\
& \cdot e^{-j k\left[\left(x_{n}-x_{n s}\right) \cos \phi+\left(z_{n}-z_{n s}\right) \sin \phi\right]}
\end{aligned}
$$

with $F_{n}(\phi)=0$ for $n \leq N_{s}+1$ in the forward sweep. In (A.14), ns $=n-$ $N_{s}-1$ is the new source point introduced in the weak group as the iterative procedure sweeps the surface in the forward direction. Namely, (A.14) shows that $F_{n}(\phi)$ continuously updates as each new source element enters the weak interaction group in the forward sweep, and hence, the complex far field pattern at the receiving element is expressed in terms of that at the previous one. This procedure produces an $O(N)$ operational cost. An analogous procedure is used for the backward propagation.

Once the integrands $F_{n}$ have been determined, it is necessary to describe the parameters which define the numerical integration in the complex space. The integration path and the numerical sampling density are described in the following subsections.

\subsubsection{The Deformed Contour of Integration}

In order to further improve efficiency of the spectral expansion, the angular spectral integration path is deformed in the complex angular plane to obtain a smaller domain of integration with less rapid oscillations along the path. 
As stated before, two-dimensional Green's function is expressed in terms of the second-kind Hankel function with order zero as,

$$
G\left(\boldsymbol{\rho}, \boldsymbol{\rho}^{\prime}\right)=\frac{1}{4 j} H_{0}^{(2)}(k R)
$$

where

$$
R=\sqrt{\left[x(\boldsymbol{\rho})-x\left(\boldsymbol{\rho}^{\prime}\right)\right]^{2}+\left[z(\boldsymbol{\rho})-z\left(\boldsymbol{\rho}^{\prime}\right)\right]^{2}}
$$

and the spectral representations of 2-D Green's function and its partial derivative are given by (A.5) and (A.6), respectively. The Hankel function is analytic in the complex plane for widely separated points, and hence, the original contour of integration in (A.5) and (A.6), $C_{\phi}$, can be deformed to a steepest descent path (SDP). For a given pair of source $\left(\boldsymbol{\rho}_{m}\right)$ and observation $\left(\boldsymbol{\rho}_{n}\right)$ points, the saddle point of the SDP is given by

$$
\phi_{n m}=\tan ^{-1}\left(\frac{z_{n}-z_{m}}{x_{n}-x_{m}}\right)
$$

on the real axis. This angle for $\phi_{n m}$ represents the direct ray connecting the source and receiving elements, demonstrating that the most significant contribution arises from the lit region of the source element.

For a flat surface, such that $z_{n}=z_{m}$, the SDP passes through its saddle point at the origin as shown in Figure 3.3, and an asymptotic analysis demonstrates that most of the contribution occurs on portions of the SDP path near a saddle point on the real axis. As the distance from the saddle point increases along the SDP path, the complex values of $\phi$ on the SDP result in an exponential attenuation of the integrand in (A.5) and (A.6), so that contributions become negligible. This deformation of the integration contour can be very advantageous numerically, since the contributing region of the path is usually much smaller than the original integration path $C_{\phi}$. This results in a smaller integration interval required. Also, allowing a smaller sampling rate for calculating the integral, rapid oscillations of the integrand can be reduced. 


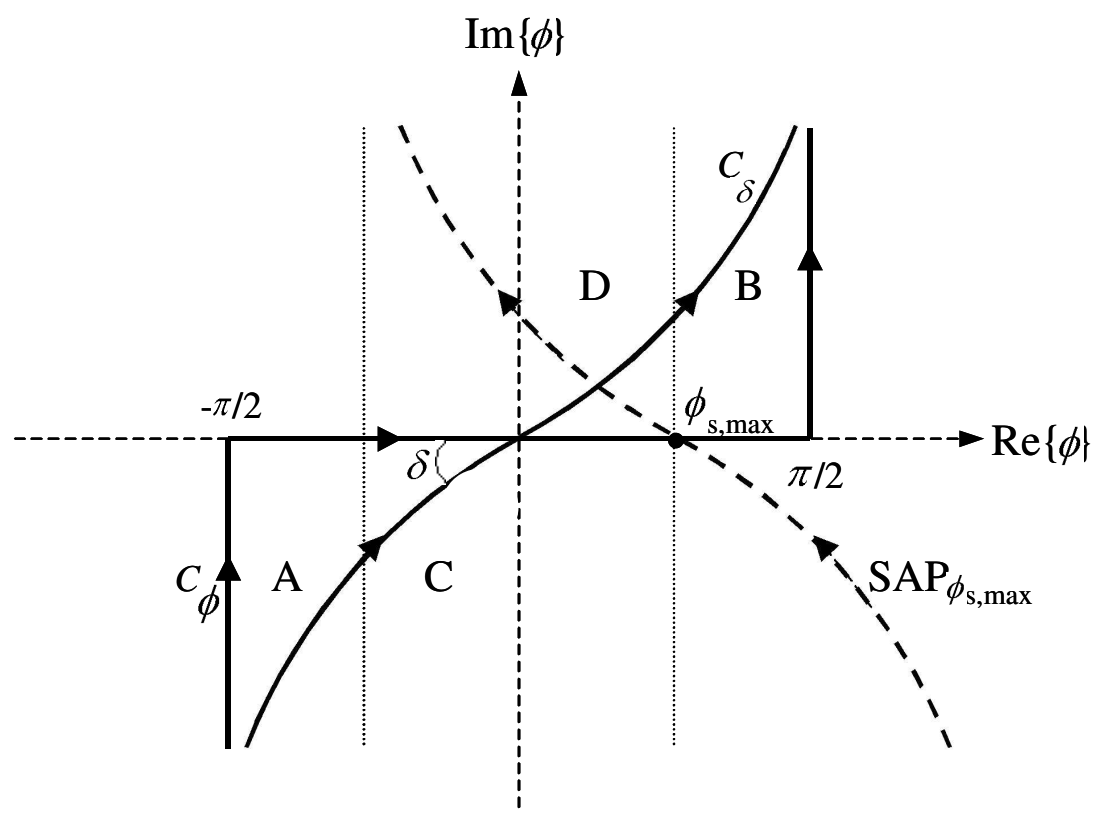

Figure 3.3: The original and deformed contours of integration.

However, when coupling between many pairs of points is considered, as in the weak region contribution to the receiving point as shown in Figure 3.1, there is no longer a unique SDP path and a single saddle point away from which only the attenuation of the integrand is obtained. In this case, several saddle points are considered on the real axis for each pair of elements, located at real values of $\phi$ given by $\phi_{n m}$ in (3.17). The width along the real axis to which these saddle points extend will be defined as the asymptotic lit region in the spatial domain, which is analogous to the geometrical optics (GO) region. Parts of the real axis outside the lit region are classified as the asymptotic shadow region in the space domain. Therefore, $\mathrm{C}$ and $\mathrm{D}$ can be defined as the lit region in the spatial domain, while $\mathrm{A}$ and $\mathrm{B}$ are defined as the shadow regions in Figure 3.3.

Since contributions between several source and receiving elements on the surface are now being considered simultaneously, there is no longer a unique SDP along which only the attenuation of the integrand is obtained away from a single saddle point. It can still be shown that the portions of all paths in 
regions $\mathrm{A}$ and $\mathrm{B}$ provide only small contributions to the integral due to either exponential attenuation or fast oscillation of the integrand. The portions of paths in regions $\mathrm{C}$ and $\mathrm{D}$ in Figure 3.3, however, now mix both descent and ascent paths where the integrand $F_{n}(\phi)$ may exponentially increase, particularly for large $z$ deviations, i.e. rougher surfaces, where the lit region becomes larger. Figure 3.4 shows this behavior by plotting the integrand along the SDP of a flat surface for different values of $x_{m}-x_{n}$ and $z_{m}-z_{n}$ values. Note that increases in the integrand away from the flat surface saddle point at $\operatorname{Re}\{\phi\}=0$ are now possible, with larger exponential increase rates for the larger values of $R$, which is given by (3.16). Consideration of the steepest ascent path (SAP) for a given saddle point shows that the maximum value along the path should occur when the SAP of the outermost saddle points intersects the contour of integration, providing a prediction method for the maximum value of the integrand obtained along a particular path.

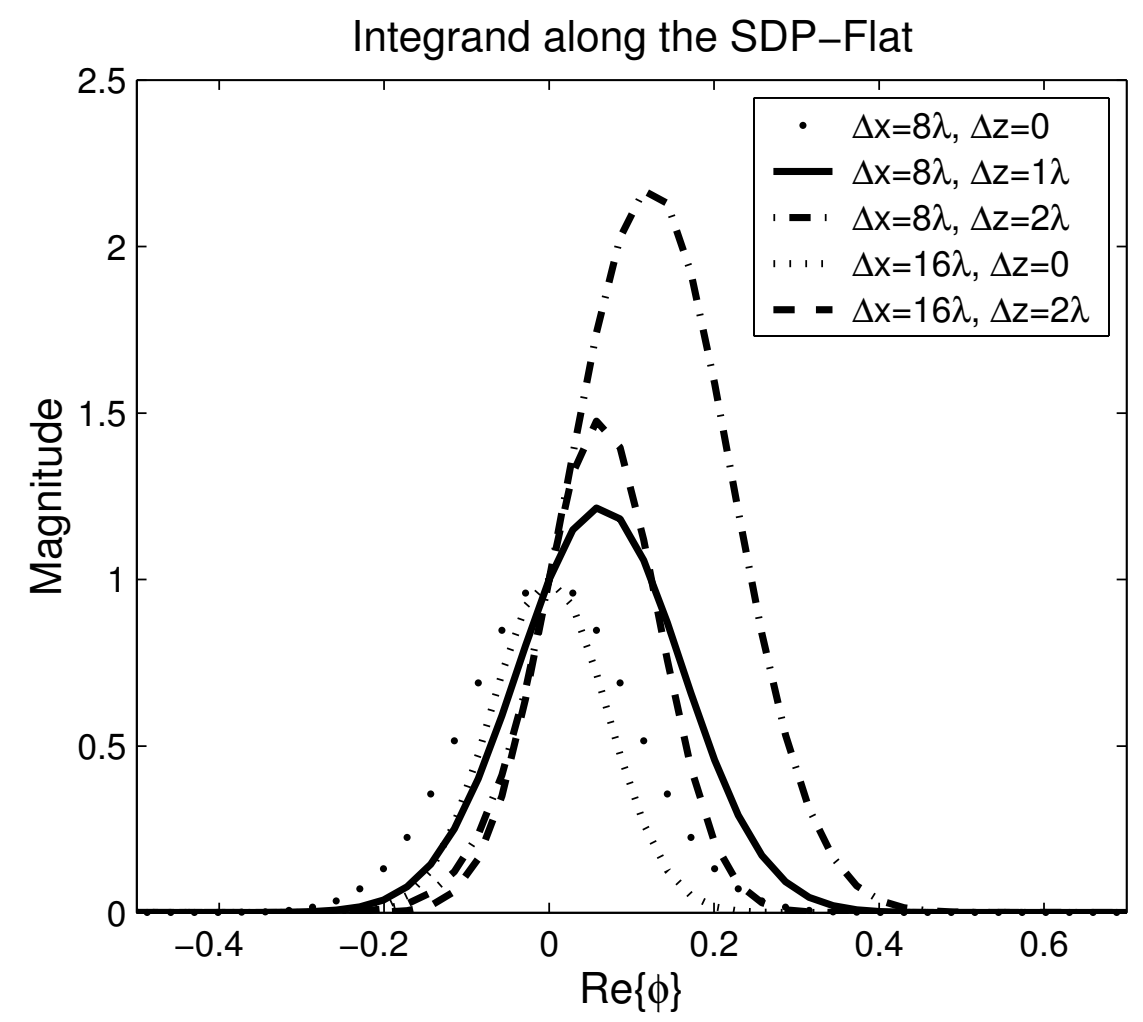

Figure 3.4: Integrand along the SDP of a flat surface. 
To avoid numerical difficulties in the integration caused by this exponential growth, it is desirable (a) to choose $L_{s}$ that yields a small spatial domain lit region, and (b) to deform the contour of integration from $C_{\phi}$ to $C_{\delta}$ which is a path between $C_{\phi}$ and the SDP for a flat surface as shown in Figure 3.3. $C_{\delta}$ is defined to be a straight line with slope $\tan \delta$ through regions $\mathrm{C}$ and D. It is noted that $\delta=\pi / 4$ for a flat surface, but this angle is chosen to be smaller for more rough surfaces in order to avoid extreme exponential growth rates in regions $\mathrm{C}$ and D. Outside the lit region, $C_{\delta}$ is deformed into the deep shadow regions so that shadow region contributions are insignificant.

One criterion for selecting $\delta$ can be obtained by limiting the maximum value of the integrand on the path to $e^{20}[7]$. The equation which results specifies $\delta=\tan ^{-1}(1 / b)$, with

$$
b=\max \left[\sqrt{\frac{k R_{s}}{20}} \phi_{s, \max }-1,1\right],
$$

which insures that the SDP of a flat surface path is obtained for flat surfaces but a smaller value of $\delta$ is used as the surface becomes more rough. In (3.18),

$$
R_{s}=\sqrt{L_{s}^{2}+\left(z_{\max }-z_{\min }\right)^{2}}
$$

and

$$
\phi_{s, \max }=\tan ^{-1}\left(\frac{z_{\max }-z_{\min }}{R_{s}}\right)
$$

are used since it is the saddle point at the shadow boundary with the largest distance between source and observation points that produces the largest possible value of the integrand.

\subsubsection{Step of Integration}

The integrals of (A.7) and (A.12) over $\phi$ can be discretized into $2 Q+1$ plane wave directions and mapped onto the real axis according to

$$
d \phi \rightarrow \Delta \phi e^{j \delta}
$$




$$
\phi \rightarrow \phi_{p}=p \Delta \phi e^{j \delta}
$$

and $Q$ can be found from [32]

$$
Q=\left\lceil\frac{2 \phi_{s, \max }}{\Delta \phi}\right\rceil
$$

where $\lceil$.$\rceil denotes the nearest integer larger than the argument. Tests for several$ rough surfaces have shown that a value of

$$
L_{s}=\frac{z_{\max }-z_{\min }}{4}
$$

can yield accurate results [7]. These selections are obtained from numerical studies of the attenuation of the integrand along the SDP of a flat surface and may not hold for very rough surfaces. An equation for $\Delta \phi$ was developed through a study of far field patterns of a uniform current distribution on an $L-L_{s}$ long flat surface and showed that approximately 44 samples are required in the main lobe region of a flat surface pattern, i.e. [7]

$$
\Delta \phi=\sqrt{\frac{5}{k R_{s}}} / 22
$$

\subsubsection{The Computational Cost of the FBSA}

The total operational count involved in finding the forward propagating radiation from the source elements can be described as follows. The number of operations for the direct numerical summation involved in the computation of strong contributions for $N$ receiving elements is $N \times N_{s}$. The operation count to compute $2 Q+1$ plane waves for $N-N_{s}$ source elements is $4(2 Q+1) \times\left(N-N_{s}\right)$, which implies three multiplications and one addition for each plane wave, and the operational count for the computation of the weak contribution from $2 Q+1$ plane waves for $N-N_{s}$ receiving elements is $2(2 Q+1) \times\left(N-N_{s}\right)$ including two multiplications for each plane wave.

As a result, the total computation cost is

$$
\text { count } \approx N \times N_{s}+6(2 Q+1) \times\left(N-N_{s}\right) .
$$


The computation count is $O(N)$ as the length increases for a fixed frequency, for large $N$. The total memory storage is also estimated as $\left(N_{s}+3\right) \times N+2 Q+1$, where $N_{s} \times N$ units are used for the storage of mutual impedances for the strong interactions group which can be eliminated if matrix elements are recalculated on each iteration, $3 N$ units are used for the incident field, forward contribution and backward contribution, and $2 Q+1$ units are required to store plane waves.

\subsection{The FBSA for Terrain Profiles}

It is important to notice that, the original implementation of the spectral acceleration algorithm, developed to analyze ocean-like rough surfaces, is only appropriate when dealing with slightly rough surfaces, becoming not suitable when dealing with very undulating geometries.

The spectral acceleration algorithm presented in [7] is modified and adapted to be able to handle wave propagation over very undulating surfaces such as terrain profiles in [21]. The most notable of these changes is the definition of a new integration path in the complex space. The operational count of this modified method is still $O(N)$, allowing to obtain the surface current distribution over real terrain profiles in a short time.

As it was stated before, the spectral acceleration algorithm is based on the fast computation of the far elements radiation by using a spectral representation of the 2-D Green's function. Thus, the radiating elements over a given receiving element are divided into two groups as the strong interaction group and the weak interaction group. With this decomposition, the fields radiated over the receiving element can be expressed as the sum of the weak and strong group contributions. Once the integrands $F_{n}$ have been determined by (A.9) and (A.14), respectively in horizontal and vertical polarization cases, as discussed in the previous section, it is necessary to describe the parameters which define the numerical integration 


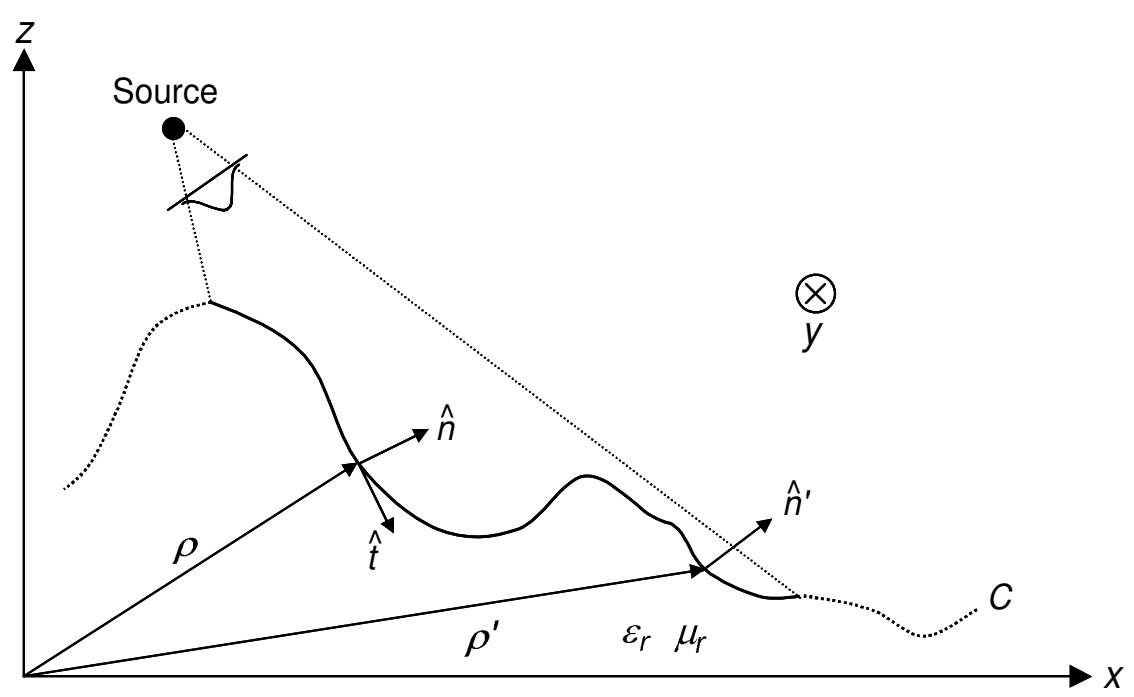

Figure 3.5: A generic terrain profile.

in the complex space. In the following subsections, the integration path and the numerical sampling density will be described.

\subsubsection{The Deformed Contour of Integration}

As explained in the previous section, the Hankel function is analytic in the complex plane for widely separated points, so the integration contour $C_{\phi}$ can be deformed to any other integration path as shown in Figure 3.6. This path is chosen to reduce the computational cost needed to evaluate the integral and to avoid possible exponential growths of the integrand values which would cause numerical errors due to the limited numerical precision of the computer.

As it can be seen in Figure 3.6, the path is composed by several stretches. The main one is the central stretch denoted by $C$; if needed two lateral stretches can be added, the left one denoted as $L$, and the right part named as $R$. All the parameters needed to determine the integration path, the angle $\delta$ that the stretch $C$ forms with the real axis and the position of $\phi_{1}, \phi_{2}, \phi_{3}$ and $\phi_{4}$ points on the complex space, will be defined next. 


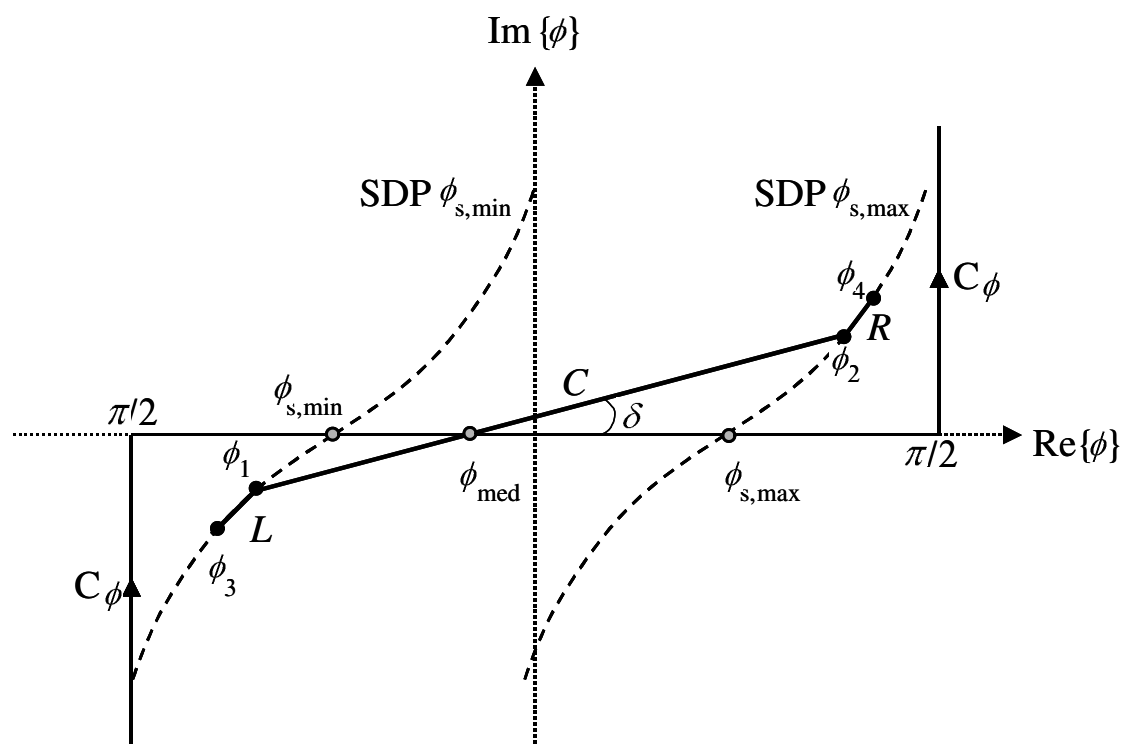

Figure 3.6: Integration path in the complex space.

To obtain these parameters, some generic considerations must be taken into account. All these considerations are related to the saddle point distribution in the complex $\phi$-space. For a general terrain profile, as one depicted in Figure 3.5, the saddle points are distributed along the real axis of the complex $\phi$ plane. Each set of source placed at $\boldsymbol{\rho}_{m}$, and receiving element placed at $\boldsymbol{\rho}_{n}$ corresponds to a saddle point located at $\phi_{n m}$ given by

$$
\phi_{n m}=\tan ^{-1}\left(\frac{z_{n}-z_{m}}{x_{n}-x_{m}}\right)
$$

such that $\phi_{n m}$ is limited by the minimum and maximum slopes of the terrain, i.e. $\phi_{n m} \in\left[\phi_{s, \min }, \phi_{s, \max }\right]$. It is important to notice that for an irregular terrain the saddle points are not distributed in a homogenous manner along the real axis. For a downhill profile, the saddle points will be placed at $\phi_{n m}<0$, whereas for an uphill geometry they will be placed at $\phi_{n m}>0$. For a generic terrain, a medium angle $\phi_{\text {med }}$ can be obtained from the mean value of all saddle point values. This angle $\phi_{m e d}$ gives a general idea of the terrain slope and the distribution of the saddle points on the real axis. It is desirable to center the integration path with respect to the saddle point distribution, so the central stretch $C$ will be centered 
at $\phi_{m e d}$, which is the cross-point of this stretch with the real axis, as shown in Figure 3.6.

To completely determine the stretch $C$, the angle $\delta$ formed with the real axis must be defined. The inclination angle of the central path $\delta$ is determined by limiting the contribution due to the most critical saddle point over the deformed contour. The limit proposed in [7], $e^{20}$, is not valid because it was obtained considering the special geometric characteristics of ocean-like rough surfaces. When the geometries under study present important height variations, this limit must be redefined. Through empirical tests it has been found that a value of $e^{2}$ is a nearly optimum choice to limit this contribution, even though smaller values can be used. Upon this consideration, the angle $\delta$ must follow this expression:

$$
\tan \delta=\min \left[\frac{1}{\sqrt{\frac{k R_{s}}{2}}\left|\phi_{n m}-\phi_{m e d}\right|-1}\right]
$$

where

$$
R_{s}=\sqrt{\left(x_{n}-x_{m}\right)^{2}+\left(z_{n}-z_{m}\right)^{2}} .
$$

It is necessary to find the worst case i.e. the maximum value of $\sqrt{R_{s}}\left|\phi_{n m}-\phi_{m e d}\right|$. The computation of $\sqrt{R_{s}}\left|\phi_{n m}-\phi_{m e d}\right|$ for all possible source-observation points sets would imply a cost of $O\left(N^{2}\right)$. For the most cases the terrain can be approximated by a coarser polygonal line with segments with dimensions proportional to the strong group length $L_{s}$. With this approximation the minimum value of $\delta$ can be obtained by analyzing the saddle point distribution over a polygonal approach to the real terrain where only the endpoints of each segment are considered.

As it can be seen by inspection of Figure 3.6, the central stretch $C$ is limited by the cross point $\phi_{1}$ with the steepest descent path of the saddle point $\phi_{s, \min }$ $\left(\operatorname{SDP} \phi_{s, \min }\right)$ and by the cross point $\phi_{2}$ with the steepest descent path of the saddle point $\phi_{s, \max }\left(\mathrm{SDP} \phi_{s, \max }\right)$. In most cases, the integrand will decay to zero near these points. However, for more complex terrains, when $\delta$ is close to $0^{\circ}$, it may be possible that some contributions do not diminish enough along stretch 
$C$, and so, $F_{n}(\phi)$ may not decay to zero near the stretch extremes. Then it would be necessary to add parts $L$ and $R$, these parts correspond to portions of the $\operatorname{SDP} \phi_{s, \min }$ and $\operatorname{SDP} \phi_{s, \max }$. Considering that these points are placed close to the real axis, the SDPs can be approximated by $45^{\circ}$ straight lines.

In case the lateral stretch $L$ is needed for a correct integration, the stretch will be extended from $\phi_{1}$ to $\phi_{3}$, a point where the integrand decays to a reference value $\zeta$. It is necessary to determine at which value of $\phi_{3}$ the contribution of $\phi_{s, \min }$ gets a value of $\zeta$ i.e.:

$$
\operatorname{Im}\left\{\phi_{3}\right\}=-\sqrt{\frac{-\ln \zeta}{k L_{s}}},
$$

where $\operatorname{Im}\{$.$\} represents the imaginary part. In general, a value of \zeta=e^{-3}$ provides a good accuracy in the complex integration, even though smaller values can be used.

An analogous procedure is used to determine the addition of the stretch $R$ considering the saddle point placed as $\phi_{s, \max }$.

\subsubsection{Step of Integration}

Once the path is determined, it is necessary to define the step of integration. For the central stretch $C$, the step of integration is defined as in the previous section, i.e.:

$$
\Delta \phi_{C}=\frac{\sqrt{5 /\left(k R_{s, \max }\right)}}{22}
$$

where

$$
R_{s, \max }=\sqrt{L_{s}^{2}+\left(z_{\max }-z_{\min }\right)^{2}}
$$

This integration step could be used for the complete integration path, but the integrand over the lateral stretches smoothly decays to zero and a larger integration step could be used. 
To determine the step used for the lateral stretches the distance $R_{s, \max }$ used in (3.31) must be substituted by the distance that defines the most significant saddle points over the lateral stretch $L$, the distance $R_{\max , \phi_{s, \min }}$ is the maximum distance between any pair of source-receiving points whose saddle point is placed at $\phi_{s, \text { min }}$. Meanwhile, for the stretch $R$, the distance $R_{\max , \phi_{s, \max }}$ is the maximum distance between any pair of source-receiving points whose saddle point is placed at $\phi_{s, \max }$. This means that the steps of integration considered are as follows: for the stretch $L$

$$
\Delta \phi_{L}=\frac{\sqrt{5 /\left(k R_{\left.\max , \phi_{s, \min }\right)}\right.}}{22}
$$

and for the lateral stretch $R$

$$
\Delta \phi_{R}=\frac{\sqrt{5 /\left(k R_{\left.\max , \phi_{s, \max }\right)}\right.}}{22}
$$

Then, the integration variable is mapped to the $\operatorname{Re}\{\phi\}$ axis according to

$$
\begin{gathered}
d \phi_{C} \rightarrow \Delta \phi_{C} e^{j \delta} \\
d \phi_{L, R} \rightarrow \Delta \phi_{L, R} e^{j \pi / 4}
\end{gathered}
$$

In some situations it could be possible that the angle $\delta$ takes very low values. This means that the path of integration will be close to the real axis such that the integrand will present very fast oscillations making it very difficult to integrate on the deformed contour. In this case, it is possible to use different integration paths along the terrain profile. Then, the complete geometry will be divided into several portions each of which can be analyzed using a different angle $\delta$ higher than a given minimum. After analyzing each portion of the geometry, a new integration path can be determined to analyze the rest of the terrain, considering that the previous saddle points will not be present for the rest of the terrain. This division process could be repeated several times along the terrain depending on the geometry. The use of different paths to analyze the whole terrain profile implies to recompute the integrand $F_{n}(\phi)$ for each new integration path, but it is done only few times so the total cost is still $O(N)$. 


\subsubsection{Computation of the Scattered Field with Spectral Acceleration}

Once the current distribution over the rough surface profile have been computed by the Forward-Backward iterative process, the next step is to compute the scattered field. If the region of interest corresponds to a small portion of the surface, the numerical evaluation of the total field will involve a reduced number of operations, but if these regions are extended to the complete terrain profile and the field strength is computed in a dense set of points, similar to the MoM discretization, the cpu-cost will increase up to $O\left(N^{2}\right)$, since the scattered field is expressed as,

$$
\begin{aligned}
E_{y}^{\text {scat }}\left(\boldsymbol{\rho}_{n}\right) & =-j \omega A_{y}-\frac{1}{\epsilon}\left\{\frac{\partial F_{x}}{\partial z}-\frac{\partial F_{z}}{\partial x}\right\} \\
& \cong-\frac{\omega \mu}{4} \sum_{m=1}^{N} I_{m} \Delta x_{m} H_{0}^{(2)}\left(k\left|\boldsymbol{\rho}_{n}-\boldsymbol{\rho}_{m}\right|\right) \\
& -j \frac{k}{4} \sum_{m=1}^{N} I_{m} \Delta x_{m} \eta_{m} H_{1}^{(2)}\left(k\left|\boldsymbol{\rho}_{n}-\boldsymbol{\rho}_{m}\right|\right) \hat{n}_{m} \cdot \hat{\rho}_{n m}
\end{aligned}
$$

and

$$
\begin{aligned}
H_{y}^{\text {scat }}\left(\boldsymbol{\rho}_{n}\right) & =-j \omega F_{y}+\frac{1}{\mu}\left\{\frac{\partial A_{x}}{\partial z}-\frac{\partial A_{z}}{\partial x}\right\} \\
& \cong \frac{\omega \epsilon}{4} \sum_{m=1}^{N} I_{m} \Delta x_{m} \eta_{m} H_{0}^{(2)}\left(k\left|\boldsymbol{\rho}_{n}-\boldsymbol{\rho}_{m}\right|\right) \\
& +j \frac{k}{4} \sum_{m=1}^{N} I_{m} \Delta x_{m} H_{1}^{(2)}\left(k\left|\boldsymbol{\rho}_{n}-\boldsymbol{\rho}_{m}\right|\right) \hat{n}_{m} \cdot \hat{\rho}_{n m}
\end{aligned}
$$

for TM and TE polarization cases respectively. Here $I_{m}$ denotes the computed induced current on the source point $\boldsymbol{\rho}_{m}$, and $\boldsymbol{\rho}_{n}$ denotes the observation point on where the scattered field will be obtained.

This $O\left(N^{2}\right)$ cost can make the method unsuitable for large terrain profiles. To overcome this limitation, the spectral acceleration can be applied to compute the scattered field only with an $O(N)$ cost. 


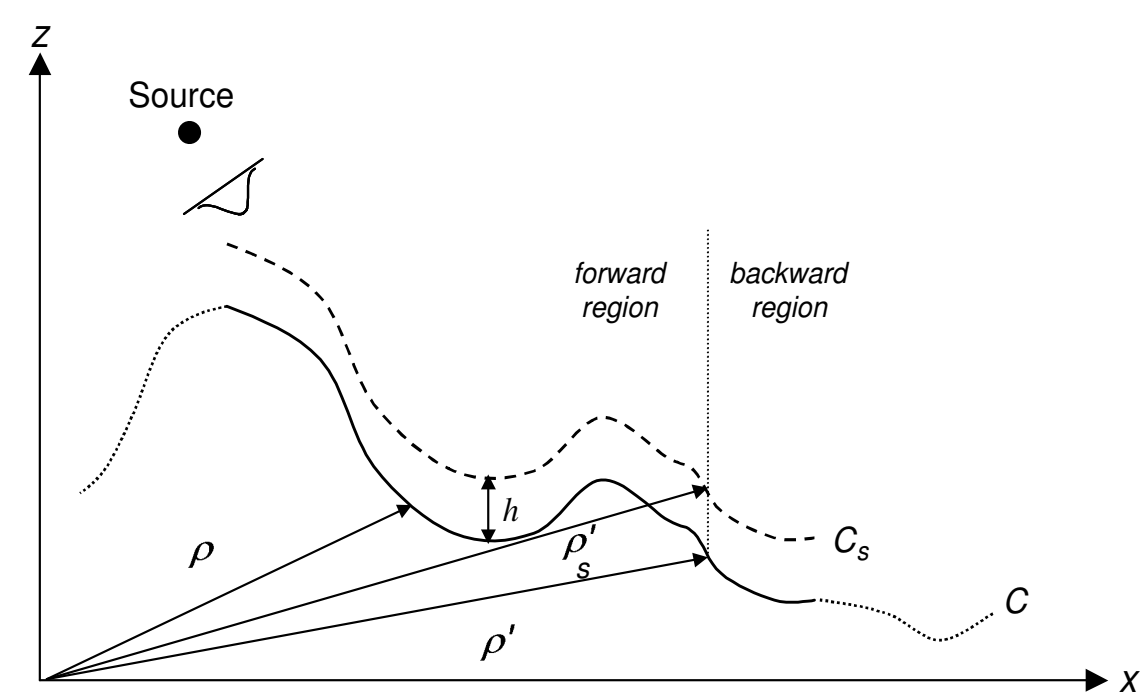

Figure 3.7: A generic terrain profile and scattering zone.

Consider the terrain profile $C$ depicted in Figure 3.7 and consider a copy of that profile $C_{s}$, where the scattered field is going to be computed, sited $h$ meters above the original terrain.

In order to make use of the spectral acceleration in computing the scattered field, the contribution of radiating elements is divided into two groups by separating the elements as forward and backward regions as shown in Figure 3.7. With this decomposition, the scattered field can be expressed as:

$$
E^{s}\left(\boldsymbol{\rho}_{k}^{s}\right)=E_{f}^{s}\left(\boldsymbol{\rho}_{k}^{s}\right)+E_{b}^{s}\left(\boldsymbol{\rho}_{k}^{s}\right)
$$

where $E_{f}^{s}\left(\boldsymbol{\rho}_{k}^{s}\right)$ and $E_{b}^{s}\left(\boldsymbol{\rho}_{k}^{s}\right)$ denote the contribution of the forward and backward regions to the scattered field in $\boldsymbol{\rho}_{k}^{s}$, respectively.

These two components of the scattered field will be computed separately. First, $E_{f}^{s}\left(\boldsymbol{\rho}_{k}^{s}\right)$ will be evaluated sweeping the terrain in the forward direction, and then $E_{b}^{s}\left(\boldsymbol{\rho}_{k}^{s}\right)$ can be obtained through a backward sweep. This division is necessary for the spectral acceleration. 
Following the explanation in Section 3.1, each component can be divided into a strong and a weak group and one can use the spectral acceleration to compute the weak group contribution. In this way, $E_{f}^{s}\left(\boldsymbol{\rho}_{k}^{s}\right)$ can be easily obtained from $E_{f}^{s}\left(\boldsymbol{\rho}_{k-1}^{s}\right)$, just recomputing the strong group radiation and updating the spectral representation of the weak group radiation. The same procedure can be done for $E_{b}^{s}\left(\boldsymbol{\rho}_{k}^{s}\right)$. With these decompositions, the scattered field can be easily calculated just sweeping the terrain forward and backward once, with a computational cost of $O(N)$.

\subsection{Numerical Results for the FBSA}

In this section, numerical results are presented to check the convergence and accuracy of the FBSA over different one-dimensional surface profiles. Results are obtained for both horizontal and vertical polarizations, considering the profiles representing both perfect and imperfect electric conductor surfaces.

In order to test the accuracy of the method, results are compared with the Method of Moments solution for the cases which have number of unknowns less than 2000. For the cases having more than 2000 surface unknowns, the FBM is used as the reference solution. The absolute error is used for monitoring the convergence of the FBSA in terms of the number of iterations. The absolute error of the FBSA method is defined by

$$
\text { absolute error }=\frac{\left\|\mathbf{I}^{(q)}-\mathbf{I}_{M o M}\right\|}{\left\|\mathbf{I}_{M o M}\right\|} .
$$

The results are grouped according to the type of surface profiles. Studies of the FBSA over slightly rough surfaces and terrain profiles are examined separately in Sections 3.3.1 and 3.3.2, respectively. The operating frequency is considered to be $300 \mathrm{MHz}$ for all numerical examples. Scattered fields are obtained over a copy of original surfaces sited $h=1.8 \lambda$ above. 


\subsubsection{Applications of the FBSA over Quasi-Planar Rough Surfaces}

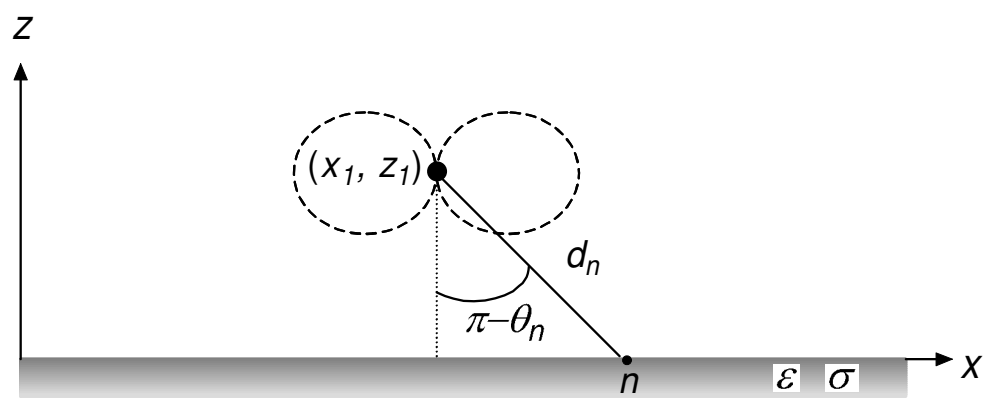

Figure 3.8: Infinitesimal Dipole on a 200 $\lambda$ strip

Consider the PEC strip profile of $w=200 \lambda$ width in Figure 3.8. This surface is considered to be illuminated by a horizontally polarized dipole antenna located symmetrically at coordinates $\left(x_{1}=100 \lambda, z_{1}=25 \lambda\right)$. For this type of source, elements of the incident field vector can be given by,

$$
V_{n}=-E_{y}^{i n c}\left(\boldsymbol{\rho}_{n}\right)=-E_{0} \frac{e^{-j k d_{n}}}{d_{n}} \sin \theta_{n}
$$

where $\theta_{n}$ is the elevation angle of the receiving element from the vertical axis of the source, and $\sin \theta_{n}$ can be determined for the strip geometry by,

$$
\sin \theta_{n}=\frac{x_{n}-x_{1}}{d_{n}}
$$

and

$$
E_{0}=\sqrt{90 P_{t}}
$$

$d_{n}$ denotes the distance between the $n$th receiving element and the source, and it is given by

$$
d_{n}=\sqrt{\left[x_{n}-x_{1}\right]^{2}+\left[z_{n}-z_{1}\right]^{2}}
$$

$P_{t}$ is the transmitted power from the horizontally polarized dipole antenna, which is considered to be 25 Watts. Choosing the pulse width as $\Delta x=\frac{\lambda}{10}$, the strip profile can be discretized into $N=2000$ segments. 
In order to test the FBSA with this geometry, the FBSA current is determined on center points of these segments and compared with the reference solution obtained via MoM. $\delta$ is selected as $\pi / 4$ since the profile is a flat surface. The neighborhood distance $L_{s}$ is taken to be $1 \lambda$, which yields to $N_{s}=10 . Q$ is computed to be 37 , so the far field pattern $F_{n}(\phi)$ consists of $2 Q+1=75$ plane waves.

(a) TM Polarized Dipole on PEC Strip (Symmetric)
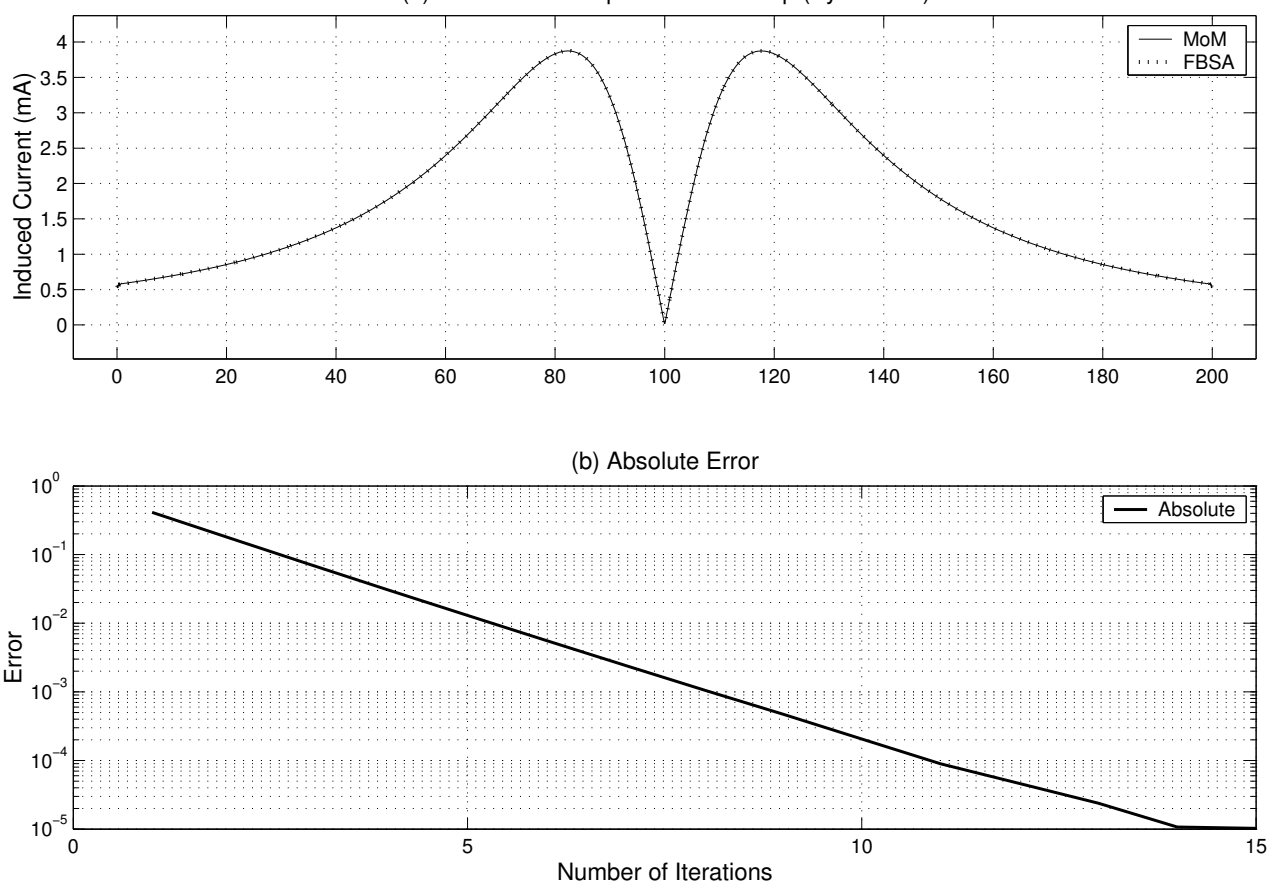

Figure 3.9: Induced current on a 200 $\lambda$ strip and absolute error (TM)

Figure 3.9 illustrates that FBSA results suit very well to the MoM reference solutions. In order to show the accuracy of the method, the absolute error versus the number of iteration graph is plotted in Figure 3.9 (b). It is clearly observed that, after six or seven iterations, the absolute error reaches to a value about $10^{-3}$.

Figure 3.10 (a) shows a $w=200 \lambda$ non-PEC quasi-planar surface profile having a maximum height deviation of $\Delta z_{\max }=z_{\max }-z_{\min }=1.17 \lambda$, which is illuminated by an infinitesimal dipole antenna located non-symmetrically over 
the profile at a height above $25 \lambda$ at the beginning of the surface. Assuming a horizontal polarization, and using (2.18) and (2.19), the entries of the impedance matrix are evaluated. (3.41)-(3.43) are valid for computing the elements of $V_{n}$ for the horizontal polarization case.
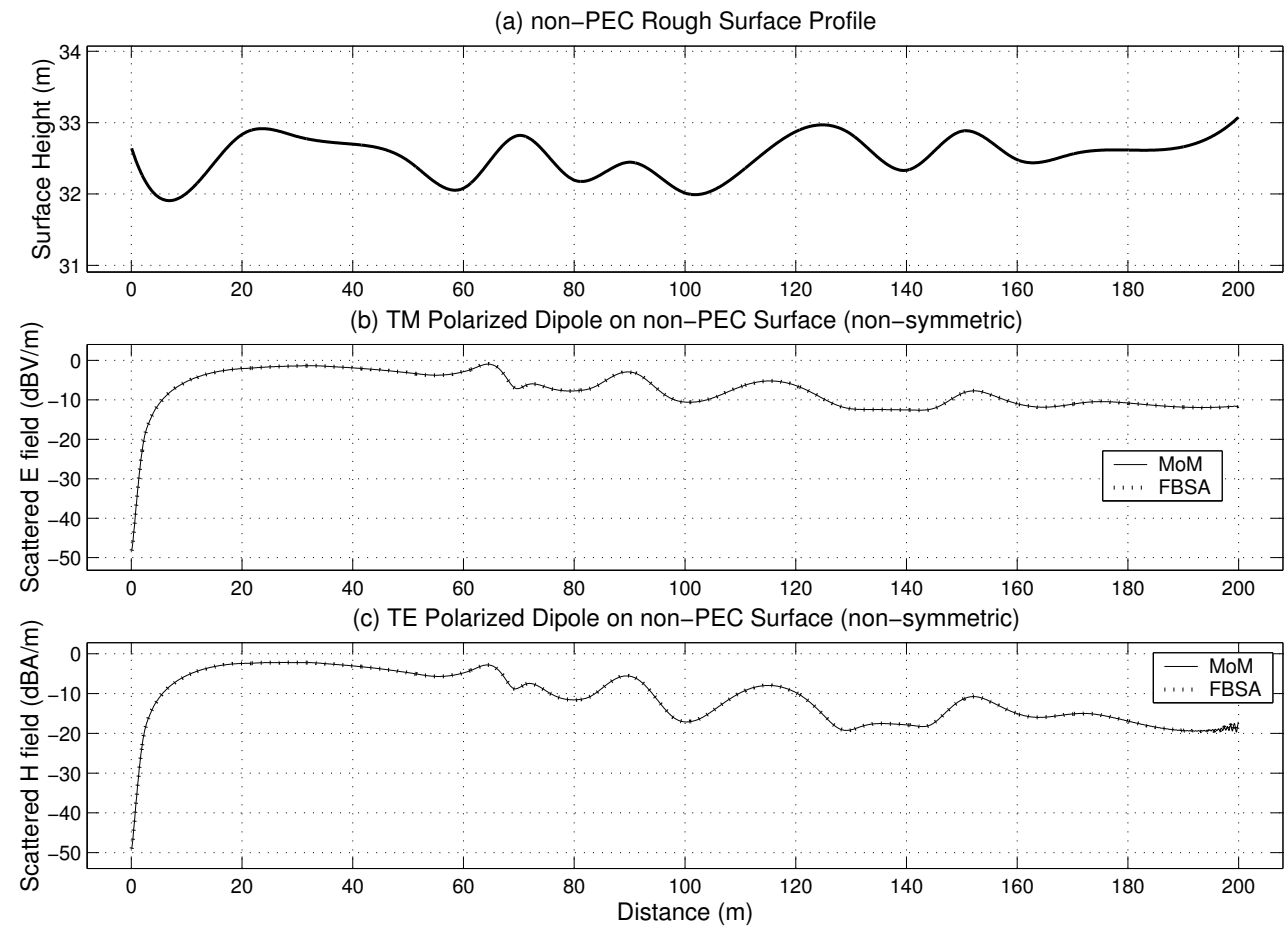

Figure 3.10: Scattered field from a $200 \lambda$ rough surface

In the case of vertical polarization, the impedance matrix elements are obtained using (2.34) and (2.35). Elements of the vector $\mathbf{V}$ are considered to be

$$
V_{n}=-H_{y}^{i n c}\left(\boldsymbol{\rho}_{n}\right)=-\frac{E_{0}}{\eta_{o}} \frac{e^{-j k d_{n}}}{d_{n}} \sin \theta_{n}
$$

for the consistency in the amplitude of the induced current, where

$$
\sin \theta_{n}=\frac{x_{n}-x_{1}}{d_{n}}
$$

and

$$
E_{0}=\sqrt{90 P_{t}}
$$

$d_{n}$ denotes the distance between the $n$th receiving element and the source, and it is given by (3.44). $P_{t}$ represents the transmitted power from the infinitesimal 
dipole in (3.47) and it is considered to be 25 Watts. $\delta$ is computed as $\pi / 4$. The neighborhood distance $L_{s}$ is taken to be $1 \lambda$, which yields $N_{s}=10 . Q$ is computed to be 44 , so the far field pattern $F_{n}(\phi)$ consists of $2 Q+1=89$ plane waves. Figure 3.10 (b) and (c) illustrate the comparison of scattered fields obtained using MoM and FBSA on $200 \lambda$ non-PEC $\left(\eta_{s}=20+j 20 \Omega\right)$ rough surface profile for nonsymmetric incident TM and TE polarized infinitesimal dipole cases, respectively. It is obvious that the FBSA presents very accurate results in both TM and TE polarization cases. The effects of shadowed regions on the induced current are clearly observed. The absolute error versus the number of iteration graphs are illustrated in Figure 3.11.

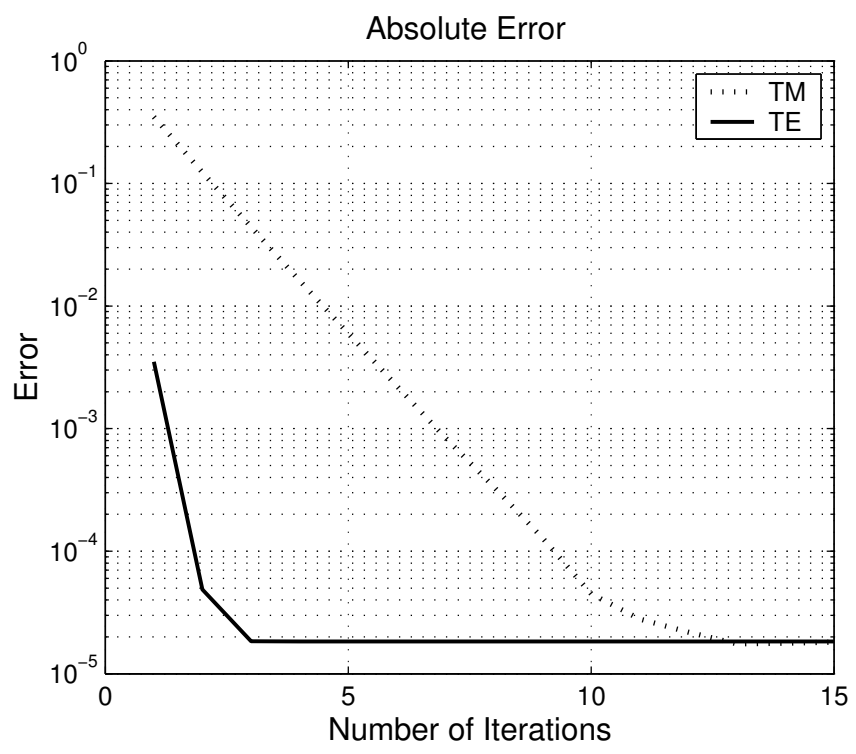

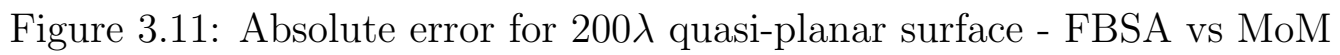

In the vertical polarization case the required number of iterations to reach a desirable error level, i.e. about $10^{-3}$, is 2 , while it is 6 in the case of horizontal polarization. The impedance matrix in the TE case is much more diagonally dominant than that of a TM case, so that the FBSA presents more accurate results within much less number of iterations in the TE case. It is also observed that the absolute error falls exponentially, but then it levels out. This is often a 
characteristic of iterative methods in general and is caused by the finite numerical precision of the computer [26].

Obtaining the MoM reference solution becomes a cumbersome problem because of the $O\left(N^{3}\right)$ computational cost, after 2000 surface unknowns. However, tests of the FBM shows that the FBM solution can be used as a numerically accurate reference for computing the scattering from rough surface profiles. The accuracy of the FBSA is tested using the FBM as a reference after 2000 unknowns.

Consider the PEC rough surface profile with a width of $w=500 \lambda$ and having a maximum height deviation of $\Delta z_{\max }=z_{\max }-z_{\min }=1.32 \lambda$, excited by a normal incident tapered plane wave, as illustrated in Figure 3.12 (a). If the incident field is horizontally polarized, elements of the vector $\mathbf{V}$ become

$$
V_{n}=-E_{y}^{i n c}\left(\boldsymbol{\rho}_{n}\right)= \begin{cases}e^{-j k\left(x_{n} \cos \theta-z_{n} \sin \theta\right)} & , \text { if } 0<x_{n}<w \\ 0 & , \text { otherwise }\end{cases}
$$

where $\theta$ is the angle of incidence from $x$-axis. The normal incidence case is considered taking the incidence angle $\theta=\frac{\pi}{2}$. In the case of vertical polarization, elements of the vector $\mathbf{V}$ are considered to be

$$
V_{n}=-H_{y}^{i n c}\left(\boldsymbol{\rho}_{n}\right)=\frac{1}{\eta_{o}} \begin{cases}e^{-j k\left(x_{n} \cos \theta-z_{n} \sin \theta\right)} & , \text { if } 0<x_{n}<w \\ 0 & , \text { otherwise }\end{cases}
$$

for the consistency in the amplitude of the induced current.

In order to test the FBSA with this geometry, the FBSA current is determined on center points of these segments and compared with the reference solution obtained via FBM. $\delta$ is computed as $\pi / 4$. The neighborhood distance $L_{s}$ is taken to be $1 \lambda$, which yields to $N_{s}=10 . Q$ is found to be 45 , so the far field pattern $F_{n}(\phi)$ consists of $2 Q+1=91$ plane waves. Figure 3.12 (b) and (c) illustrate the scattered fields obtained using FBSA and FBM after 6 iterations for TM polarization and after 2 iterations for TE polarization on 500 $\lambda$ PEC 
$\left(\eta_{s}=0\right)$ rough surface profile, respectively. The absolute error versus the number of iteration graphs are illustrated in Figure 3.13.

Figure 3.14 (a) shows a $w=1000 \lambda$ non-PEC quasi-planar surface profile having a maximum height deviation of $\Delta z_{\max }=z_{\max }-z_{\min }=1.53 \lambda$, which is illuminated by a isotropic radiator located non-symmetrically over the profile at a height above $25 \lambda$ in the beginning of the surface. Assuming a horizontal polarization, and using (2.18) and (2.19), the entries of the impedance matrix are evaluated. For this type of source, elements of the incident field vector can be given by,

$$
V_{n}=-E_{y}^{i n c}\left(\boldsymbol{\rho}_{n}\right)=-E_{0} \frac{e^{-j k d_{n}}}{d_{n}}
$$

where $d_{n}$ denotes the distance between the $n$th receiving element and the source, and it is given by (3.44) and

$$
E_{0}=\sqrt{60 P_{t}}
$$

In the case of vertical polarization, the impedance matrix elements are obtained using (2.34) and (2.35). Elements of the vector $\mathbf{V}$ are considered to be

$$
V_{n}=-H_{y}^{i n c}\left(\boldsymbol{\rho}_{n}\right)=-\frac{E_{0}}{\eta_{o}} \frac{e^{-j k d_{n}}}{d_{n}}
$$

for the consistency in the amplitude of the induced current, and

$$
E_{0}=\sqrt{60 P_{t}}
$$

$P_{t}$ denotes the transmitted power from the isotropic radiator in (3.53) and it is considered to be 25 Watts.

For this geometry, $\delta$ is computed as $\pi / 4$. The neighborhood distance $L_{s}$ is taken to be $1 \lambda$, which yields $N_{s}=10 . Q$ is computed to be 48 , so the far field pattern $F_{n}(\phi)$ consists of $2 Q+1=97$ plane waves. Figure 3.14 (b) and (c) illustrate the comparison of scattered fields obtained using FBM and FBSA on $1000 \lambda$ non-PEC $\left(\eta_{s}=20+j 15 \Omega\right)$ rough surface profile for nonsymmetric incident TM and TE polarized isotropic radiator cases, respectively. The absolute error versus the number of iteration graphs are illustrated in Figure 3.15. 
(a) PEC Rough Surface Profile

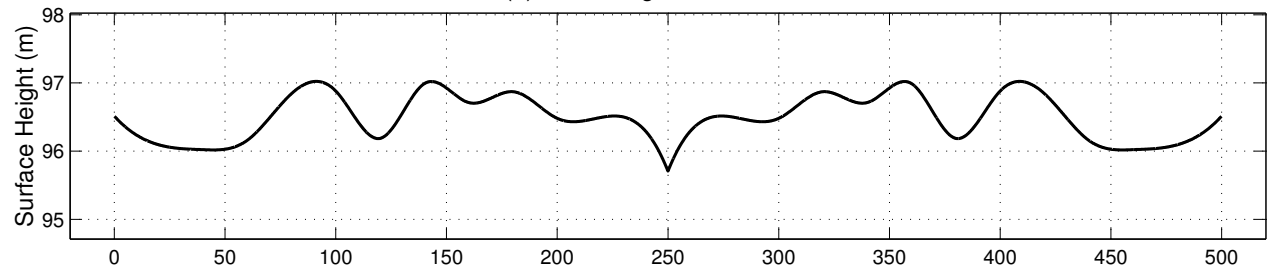

(b) TM Polarized PlaneWave on PEC Surface (Normal)

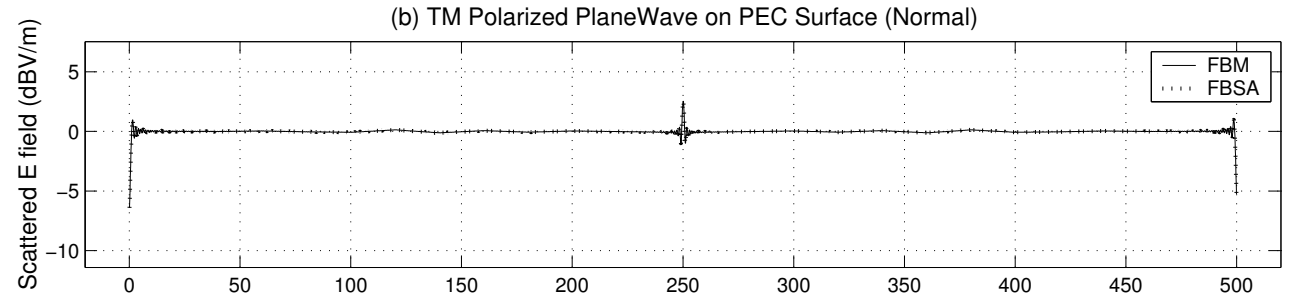

(c) TE Polarized PlaneWave on PEC Surface (Normal)

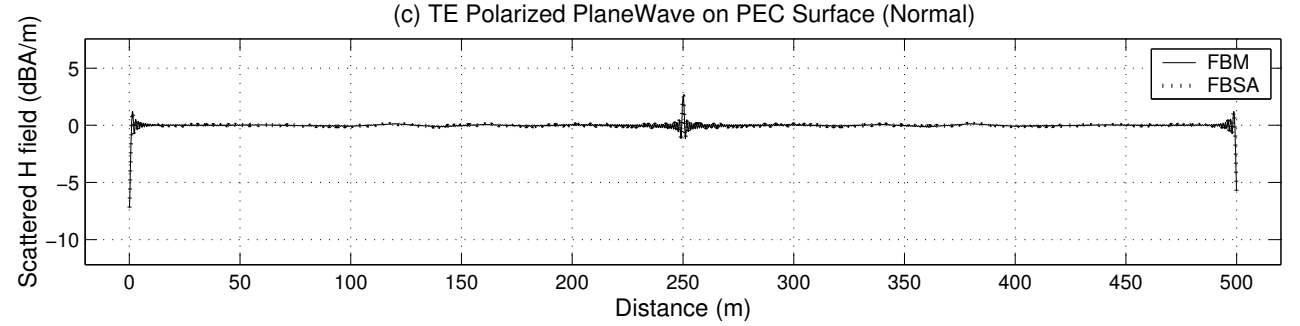

Figure 3.12: Scattered field from a $500 \lambda$ rough surface

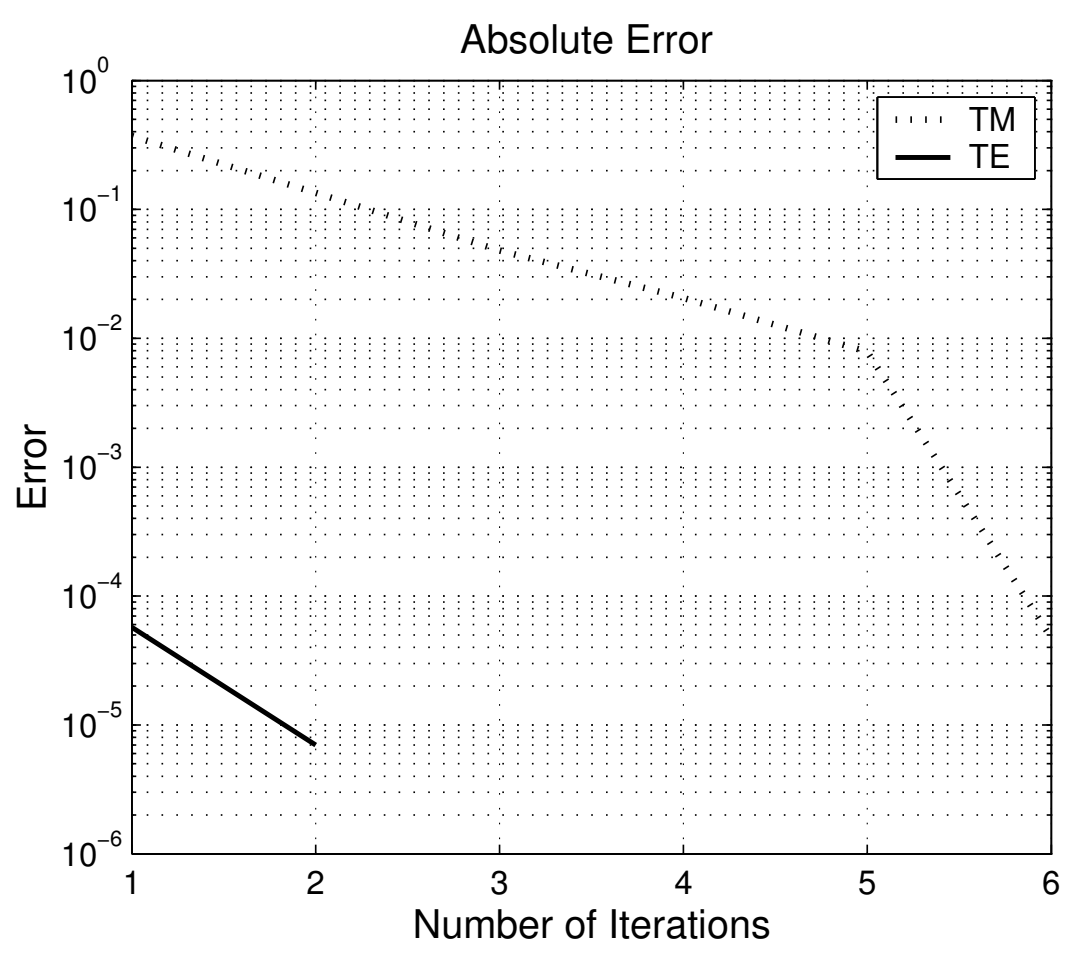

Figure 3.13: Absolute error for 500 $\lambda$ quasi-planar surface - FBSA vs FBM 
(a) non-PEC Rough Surface Profile

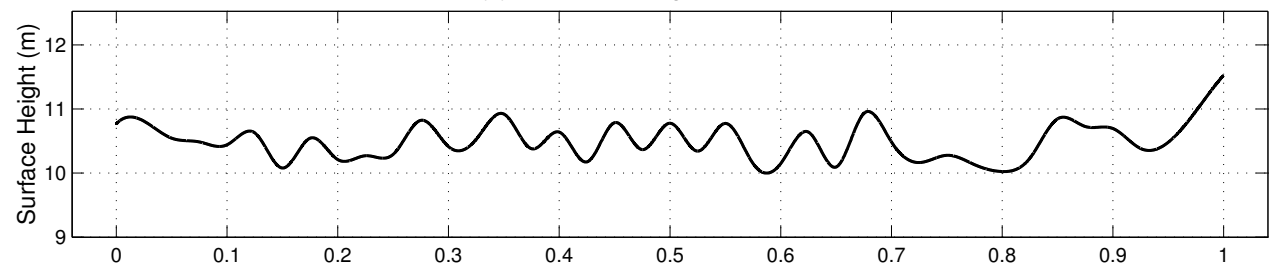

(b) TM Polarized Isotropic Source on non-PEC Surface (non-symmetric)

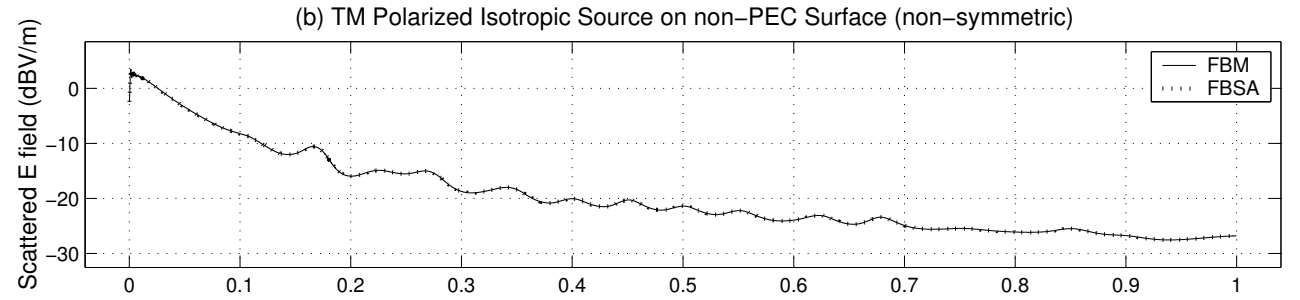

(c) TE Polarized Isotropic Source on non-PEC Surface (non-symmetric)

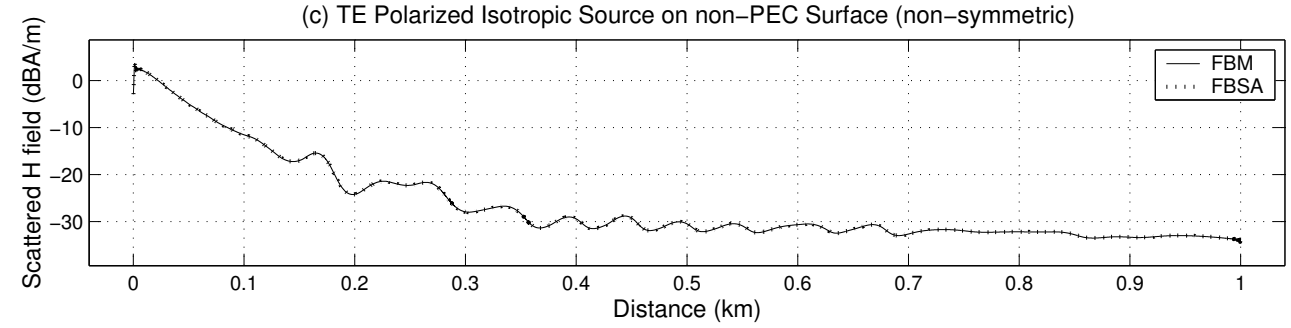

Figure 3.14: Scattered field from a 1000 $\lambda$ rough surface

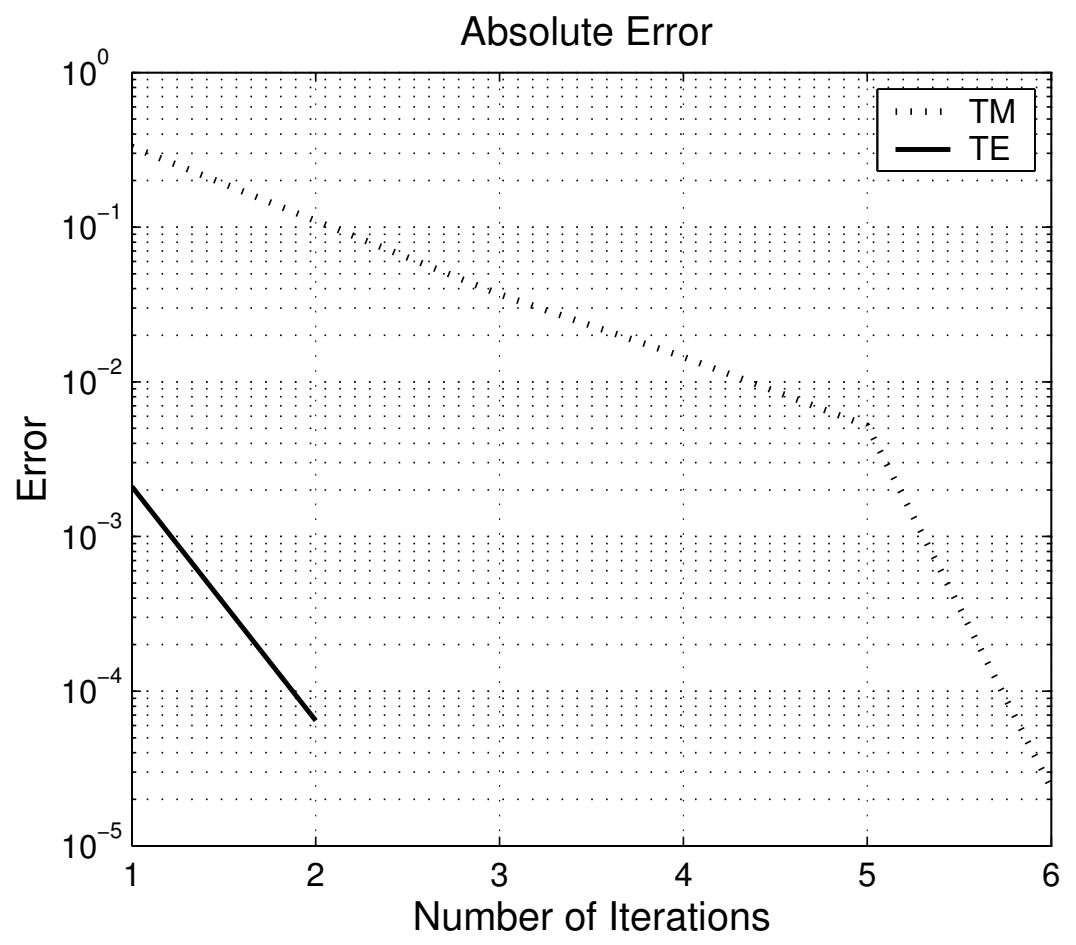

Figure 3.15: Absolute error for 1000ג QP surface - FBSA vs FBM 
Obtaining the FBM reference solution becomes a cumbersome problem because of the $O\left(N^{2}\right)$ computational cost, after 20000 surface unknowns. However, tests of the FBSA shows that the FBSA solution can be used as a numerically accurate reference for computing the scattering from quasi-planar rough surface profiles.
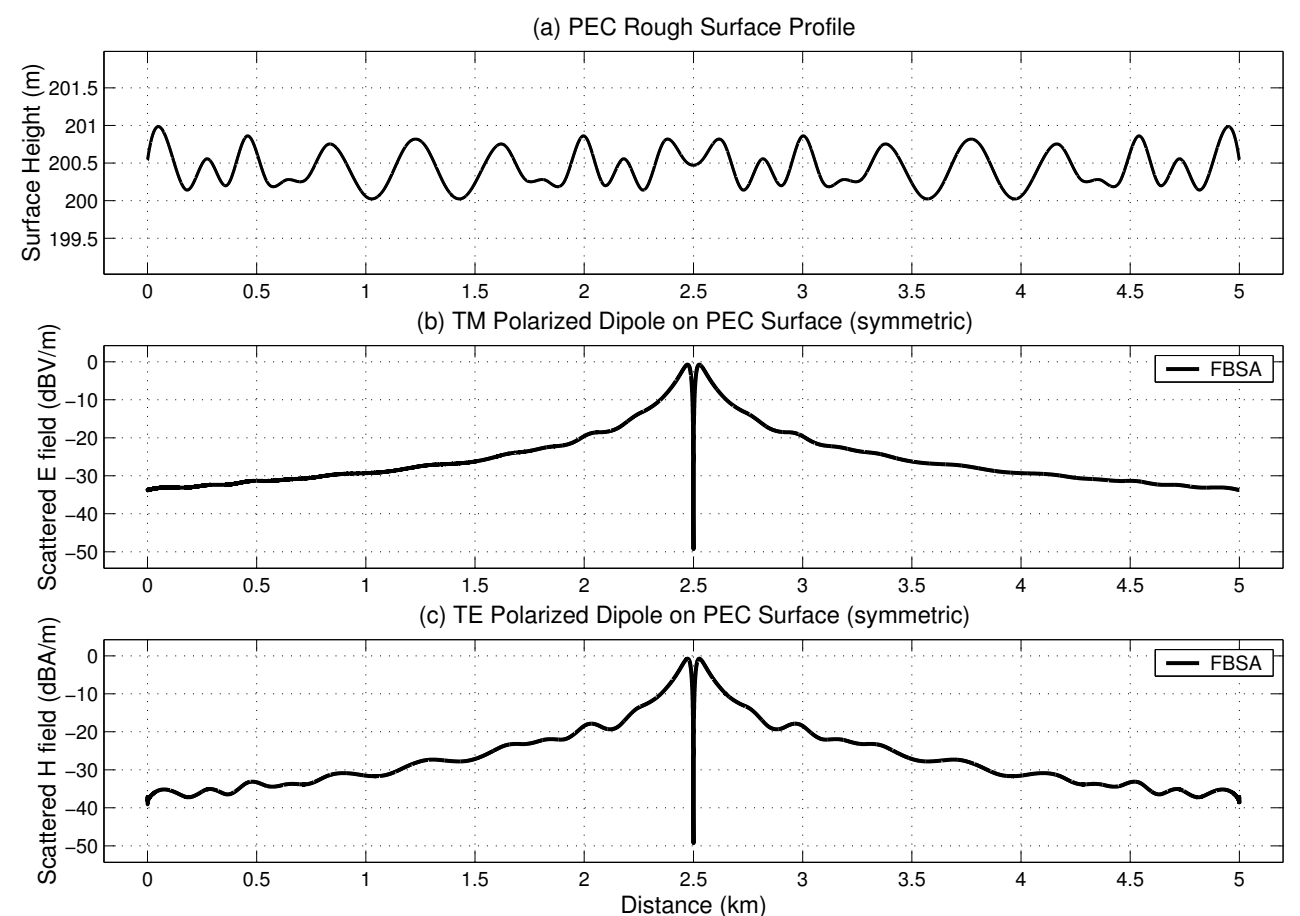

Figure 3.16: Scattered field from a 5000 $\lambda$ rough surface

Consider the PEC rough surface profile with a width of $w=5000 \lambda$ and having a maximum height deviation of $\Delta z_{\max }=z_{\max }-z_{\min }=0.97 \lambda$ in Figure 3.16 (a). This surface is considered to be illuminated by a dipole antenna located symmetrically at a height above $25 \lambda$ in the middle of the $x$ axis of the surface. (3.41)-(3.43) and (3.45)-(3.47) are valid for computing the elements of $V_{n}$ for the horizontal and vertical polarization cases, respectively. For this geometry, $\delta$ is computed as $\pi / 4$. The neighborhood distance $L_{s}$ is taken to be $1 \lambda$, which yields $N_{s}=10 . Q$ is computed to be 42 , so the far field pattern $F_{n}(\phi)$ consists of $2 Q+1=85$ plane waves. Figure 3.16 (b) and (c) illustrate the comparison of scattered fields obtained using FBSA on 5000 $\lambda$ PEC $\left(\eta_{s}=0\right)$ rough surface 


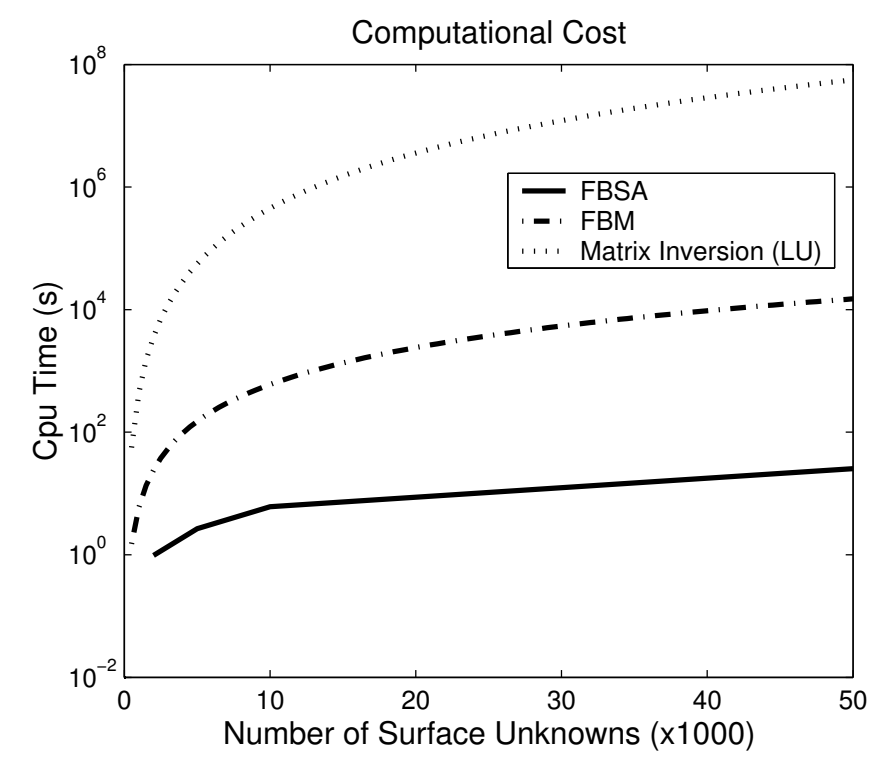

Figure 3.17: Computational Cost

profile for nonsymmetric incident TM and TE polarized infinitesimal dipole cases, respectively.

Investigations over rough surface profiles show that the FBSA obtains the numerically accurate solutions within very few iterations on quasi-planar rough surfaces for both horizontal and vertical polarization cases. In order to compare the computational cost of the method with MoM and the FBM, the computation time versus number of iterations examinations are illustrated in Figure 3.17 and Table 3.1 for three of the reference methods. The FBM and FBSA reach a desirable level of error after 6 iterations in the TM case and after 2 iterations in the TE case.

Note that, after 2000 unknowns, cpu-time values for the MoM are obtained using a spline interpolation of those for less than 2000. The same process is done for the FBM after 20000 surface unknowns. It is obvious that, the FBSA presents a computational cost of $O(N)$ while the direct solution of the MoM requires $O\left(N^{3}\right)$ cpu-time and the FBM causes $O\left(N^{2}\right)$, where $N$ is the total number of surface unknowns. 


\begin{tabular}{|c|c|c|c|}
\hline$N$ & LU Inversion (s) & FBM per iter. (s) & FBSA per iter (s) \\
\hline 500 & 56 & 1.5 & - \\
1000 & 452 & 5.9 & - \\
2000 & 3595 & 24 & 0.98 \\
5000 & $\mathrm{NA}$ & 155 & 2.67 \\
10000 & $\mathrm{NA}$ & 612 & 6.08 \\
20000 & $\mathrm{NA}$ & 2504 & 10.94 \\
50000 & $\mathrm{NA}$ & NA & 25.5 \\
\hline
\end{tabular}

Table 3.1: Computational Cost

The comparisons demonstrate that, the FBSA can be used as a numerically accurate reference solution instead of MoM or FBM over quasi planar rough surface profiles in both horizontal and vertical polarization cases, because of its very accurate results within very few iterations, causing a computational time and a memory requirement of $O(N)$.

\section{The Limitations of the Spectral Acceleration Algorithm}

The original spectral acceleration algorithm is proposed for quasi-planar or slightly rough surface profiles as ocean-like surfaces. The algorithm is good at analyzing the scattering from such profiles of which the maximum height variation is several wavelengths. Thus, the saddle points are considered to be distributed around the origin on the complex $\phi$ space as the saddle points of a flat surface.

As the roughness of the surface increases, many saddle points will be grouped close to the outermost saddle point, $\phi_{s, \max }$, near the steepest ascent path intersecting the deformed contour. Also the $e^{20}$ term for the upper limit of the integrand in the weak region contribution expression is not a suitable limit as the roughness increases, because the weak region contribution can easily blow up for values smaller than but close to $e^{20}$. In order to overcome this problem, the limit of the integrand is proposed to be $e^{2}$ instead of $e^{20}$ in [32].

However, this suggestion does not overcome the limitation of the spectral acceleration for uphill or downhill surface geometries, since the deformed contour 
of integration is still considered to intersect the real axis of the complex $\phi$ space at the origin. For an uphill profile, most of the saddle points will be grouped around somewhere on the positive $\operatorname{Re}\{\phi\}$ axis, and maybe even around a value larger than the estimated outermost saddle point $\phi_{s, \max }$. In this case, the SDP of a flat surface or any deformed contour intersecting the $\operatorname{Re}\{\phi\}$ axis at the origin will become useless, since the main contribution comes from saddle points which are distributed around somewhere else.

Figure 3.18 shows an impedance $\left(\eta_{s}=20+15 j \Omega\right) 200 \lambda$ width uphill rough surface profile illuminated by an infinitesimal dipole located at a $25 \lambda$ height above the beginning of the surface. In the $e^{20}$ case for the upper limit of the integrand, the inclination angle is computed to be $21.2^{\circ}$ and this slope causes the deformed contour to intersect the steepest ascent path of the $\phi_{s, \max }$ and the integrand to become $e^{20}$ at the point of this intersection, and hence, the weak region term blows up immediately even in the first iteration. Consequently no converged result is obtained using the FBSA. However, for this example, using $e^{2}$ as the upper limit of the integrand, the inclination angle is found as $5.5^{\circ}$ and the strong region neighborhood is computed to be $L_{s}=17 \lambda$. The FBSA converges with an absolute error about $10 \%$ in the TM polarization case and about $5 \%$ for TE polarization when these parameters are used. Figure 3.18 shows the comparisons of the MoM and FBSA solution for both polarizations. It is obvious that the method fails to be a reference solution as the roughness increases.

Consider the non-PEC $\left(\eta_{s}=20+15 j \Omega\right)$ rough surface profile having a $500 \lambda$ width and illuminated by an infinitesimal dipole located at a $25 \lambda$ height above the beginning of the surface as illustrated in Figure3.19 (a). In the $e^{20}$ case for the upper limit of the integrand, the inclination angle is computed to be $10.9^{\circ}$ and this slope causes the deformed contour to intersect the steepest ascent path of the $\phi_{s, \max }$ and the integrand to become $e^{20}$ at the point of this intersection. Therefore, the weak region term blows up immediately even in the first iteration. 
(a) non-PEC Surface Profile

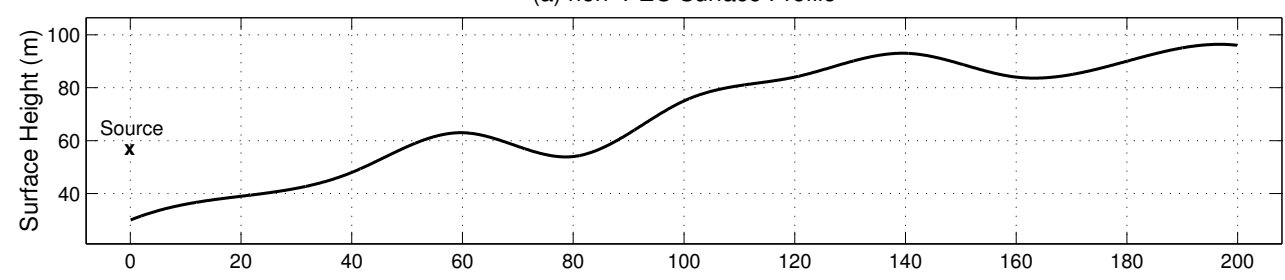

(b) TM Polarized Dipole on non-PEC Surface

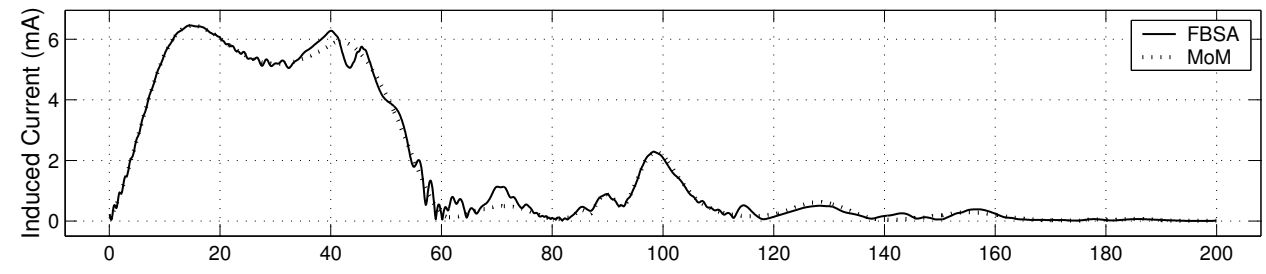

(c) TE Polarized Dipole on non-PEC Surface

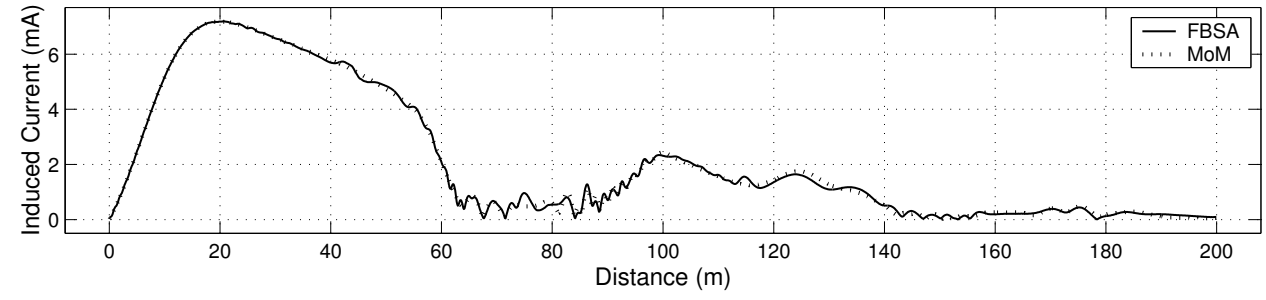

Figure 3.18: Induced Current on a 200 $\lambda$ rough surface

Consequently, no converged results are obtained using the FBSA. However, for the same example, using $e^{2}$ as the upper limit of the integrand of the FBSA, the inclination angle is found as $3.2^{\circ}$ and the strong region neighborhood is computed to be $L_{s}=50 \lambda$. Figure 3.19 shows the comparisons of the FBM and FBSA solution for both polarizations when these parameters are used. Since the inclination angle is too small in this case, the deformed contour of integration approaches close to both the real axis and its intersection with the SAP of the outermost saddle point. This point has a significant effect on the exponential growth of the integrand on the complex space. In fact, the assumption of the origin to be the most effective saddle point for the weak region contribution loses its validity as the roughness increases, and hence the inclination angle becomes too small for an uphill geometry for which the saddle points are densely distributed around somewhere on the positive real axis or a downhill geometry for which the saddle points are densely distributed around somewhere on the negative real axis. 
(a) non-PEC Surface Profile

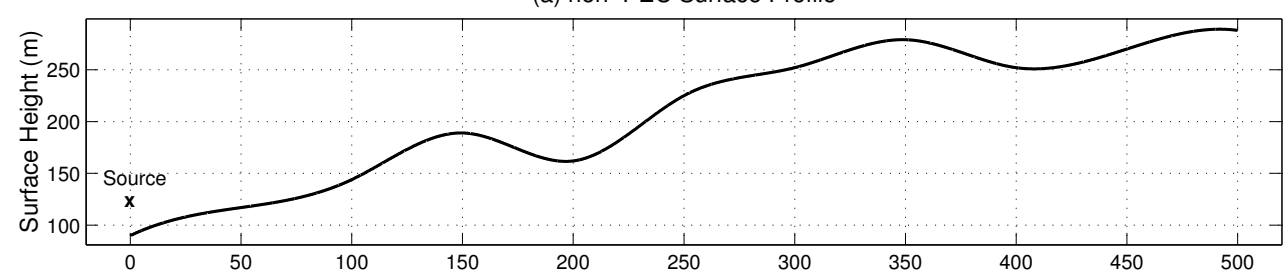

(b) TM Polarized Dipole on non-PEC Surface

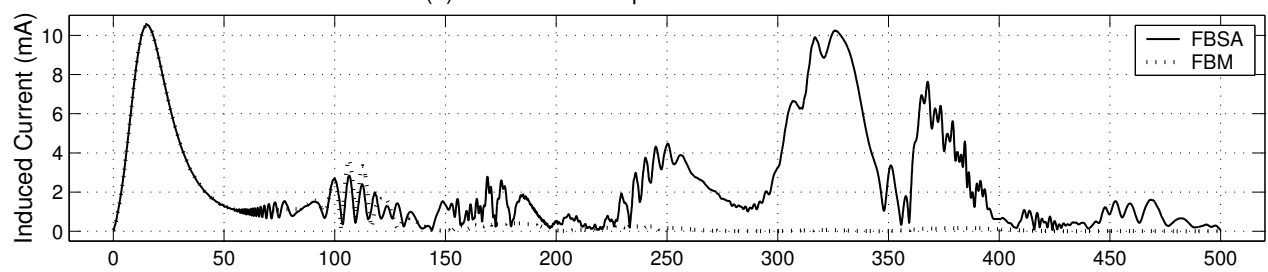

(c) TE Polarized Dipole on non-PEC Surface

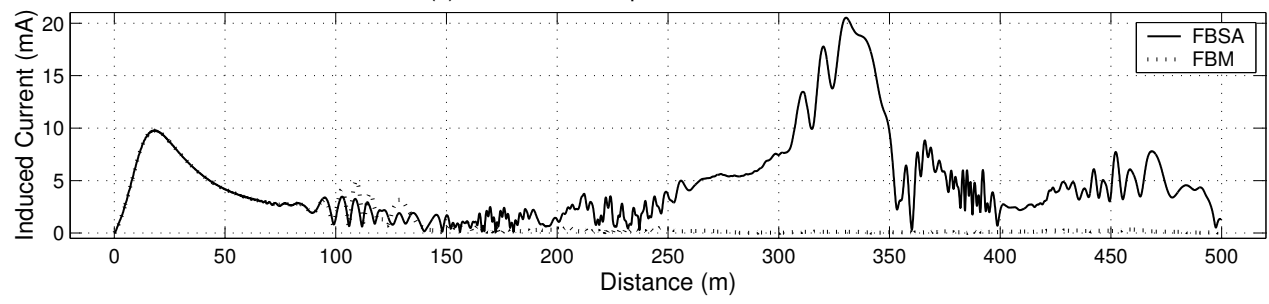

Figure 3.19: Induced Current on a $500 \lambda$ rough surface

In order to overcome this limitation, the spectral acceleration algorithm is modified to handle with very undulating rough surfaces such as terrain profiles in [21]. Applications of this modified algorithm are discussed in the next subsection.

\subsubsection{Applications of the FBSA over Terrain Profiles}

In this section, the validity of the modified spectral acceleration algorithm is shown by comparing the results with reference solutions. The scattered field values are computed, for comparisons, over a copy of each terrain, sited $h=1.8 \lambda$ above the original profile. The MoM is used as the reference solution for terrain profiles having number of unknowns less than 2000. For the cases having more than 2000 surface unknowns, numerically accurate solutions are obtained using FBM. Details about each case are given by tables and figures for every terrain profile analyzed. 
Consider $200 \lambda$ width non-PEC $\left(\eta_{s}=20+j 15 \Omega\right)$ terrain profile illustrated in Figure 3.20 (a). The parameters that are needed to implement the FBSA to this geometry are included in Table 3.2 and the deformed contour of integration is illustrated in Figure 3.20 (b). Since the geometry is a downhill profile, the medium saddle point is located on the negative real axis. This surface is considered to be illuminated by a dipole antenna located at a $25 \lambda$ height above the left-most point of the terrain. (3.41)-(3.43) and (3.45)-(3.47) are valid for computing the elements of $V_{n}$ for the horizontal and vertical polarization cases, respectively. $P_{t}$, the transmitted power from the infinitesimal dipole, is considered to be 25 Watts.

\begin{tabular}{|c|c|c|c|c|}
\hline Number of Unknowns $(N)$ & 2000 & 5000 & 10000 & 20000 \\
\hline Frequency $(\mathrm{MHz})$ & 300 & 300 & 300 & 300 \\
\hline$\phi_{\text {med }}(\mathrm{rad})$ & -0.290 & -0.076 & 0.143 & -0.013 \\
\hline$\phi_{s, \min }(\mathrm{rad})$ & -0.573 & -1.112 & -1.011 & -0.258 \\
\hline$\phi_{s, \max }(\mathrm{rad})$ & 0.511 & 0.955 & 0.896 & 0.213 \\
\hline$L_{s}(\lambda)$ & 2 & 2 & 2 & 2 \\
\hline Num. of integr. points & 315 & 715 & 812 & 300 \\
\hline$\delta(\mathrm{rad})$ & 0.264 & 0.104 & 0.112 & 0.218 \\
\hline
\end{tabular}

Table 3.2: Study Parameters

Figure 3.21 (b) and (c) illustrate the comparison of scattered fields, using MoM and FBSA on 200 $\lambda$ terrain profile illustrated in Figure 3.21 (a) for TM and TE polarized infinitesimal dipole cases, respectively. The absolute error versus the number of iteration graphs are illustrated in Figure 3.22.

Figure 3.23 (a) shows a $500 \lambda$ width PEC $\left(\eta_{s}=0\right)$ terrain profile. The study parameters of FBSA for this profile are included in Table 3.2 and the deformed contour of integration is illustrated in Figure 3.23 (b). Since the geometry is a downhill profile, the medium saddle point is located on the negative real axis. This surface is considered to be illuminated by a dipole antenna located at a $25 \lambda$ height above the middle of the terrain. 
(a) non-PEC Rough Surface Profile

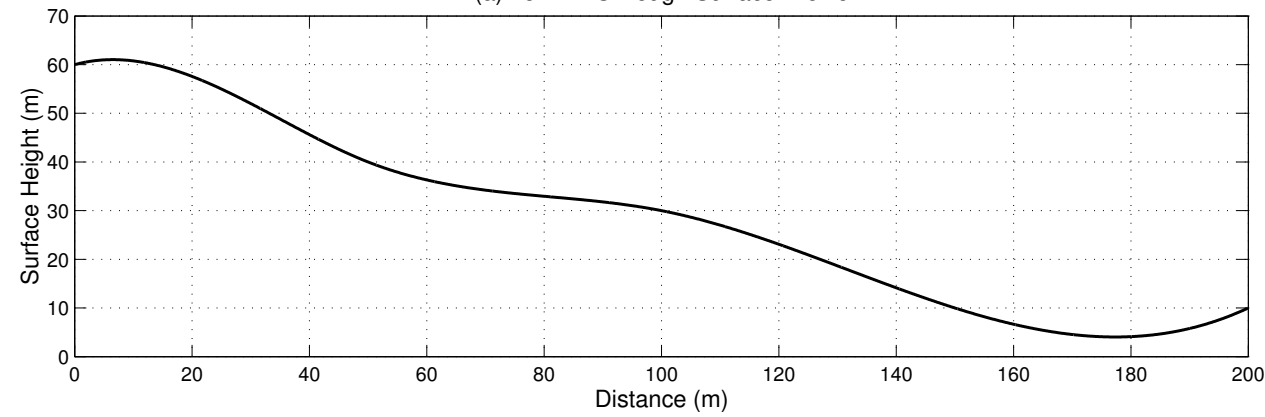

(b) Deformed Integration Contour

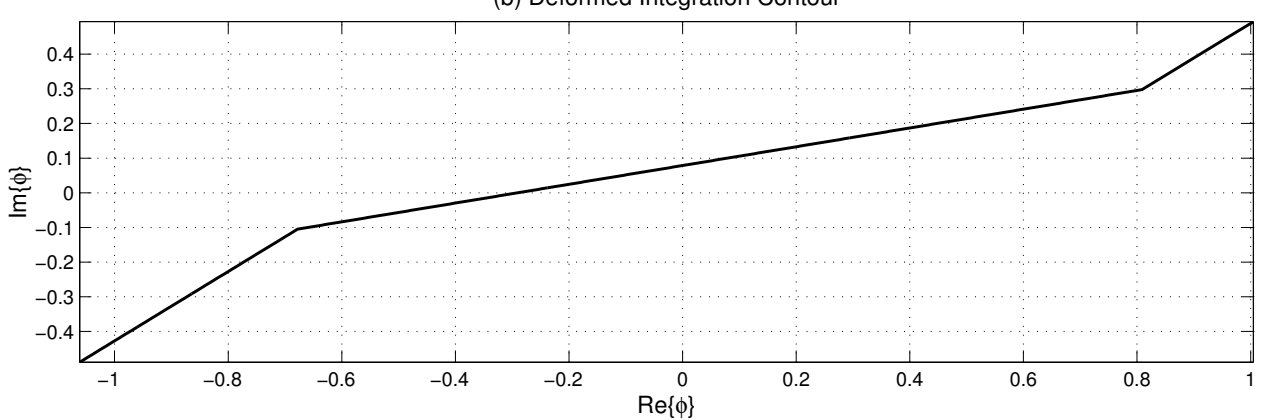

Figure 3.20: Deformed contour for a $200 \lambda$ terrain profile

(a) non-PEC Rough Surface Profile
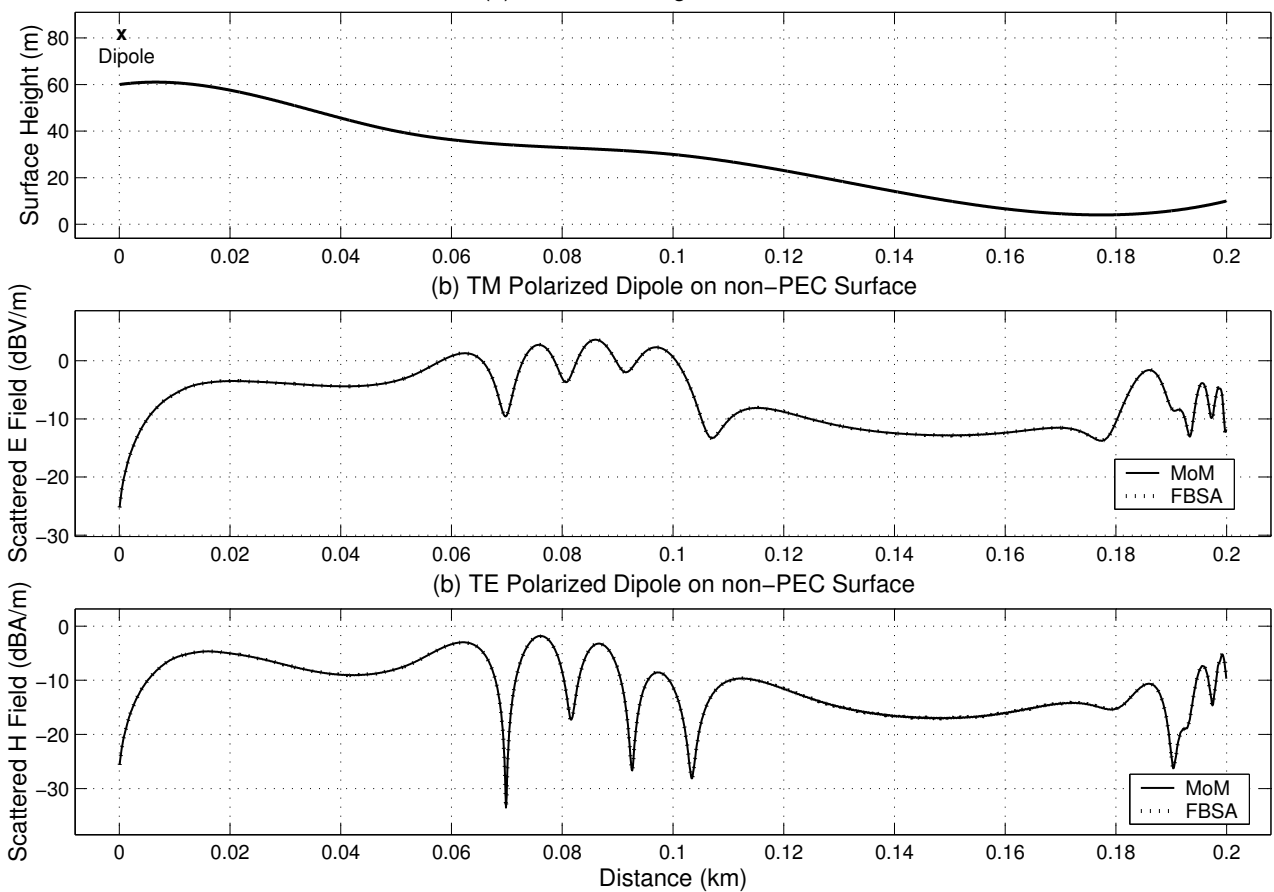

Figure 3.21: Scattered field from a $200 \lambda$ width terrain 


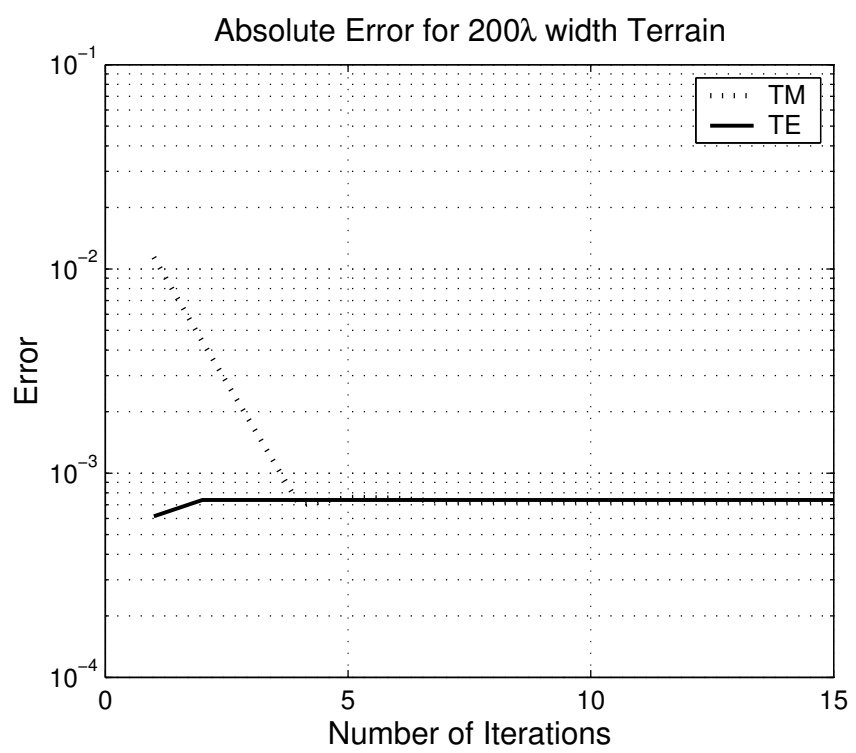

Figure 3.22: Absolute error for 200 $\lambda$ terrain - FBSA vs MoM

(3.41)-(3.43) and (3.45)-(3.47) are valid for computing the elements of $V_{n}$ for the horizontal and vertical polarization cases, respectively. $P_{t}$, the transmitted power from the infinitesimal dipole, is considered to be 40 Watts.

In order to test the FBSA with this geometry, the scattered field is determined on center points of these segments via FBSA using the corresponding parameters, and compared with the reference solution computed by FBM. The absolute error versus the number of iteration graphs, illustrated in Figure 3.27, show the accuracy of the method. Figure 3.24 (b) and (c) illustrate the comparison of scattered fields, using FBM and FBSA on $500 \lambda$ width terrain profile illustrated in Figure 3.24 (a) for TM and TE polarized infinitesimal dipole cases, respectively. The absolute error versus the number of iteration graphs are illustrated in Figure 3.27. Consider $1000 \lambda$ width non-PEC $\left(\eta_{s}=15+j 20 \Omega\right)$ terrain profile in Figure 3.25 (a). The parameters that are needed to implement the FBSA to this geometry are included in Table 3.2 and the deformed contour of integration is illustrated in Figure 3.25 (b). Since the geometry is an uphill profile, the medium saddle point is located on the positive real axis. 
(a) PEC Rough Surface Profile

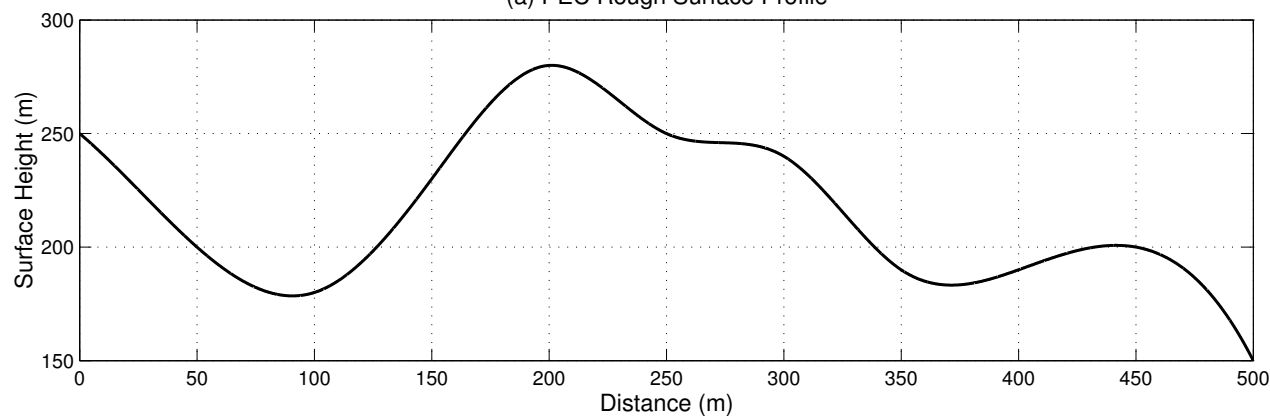

(b) Deformed Integration Contour

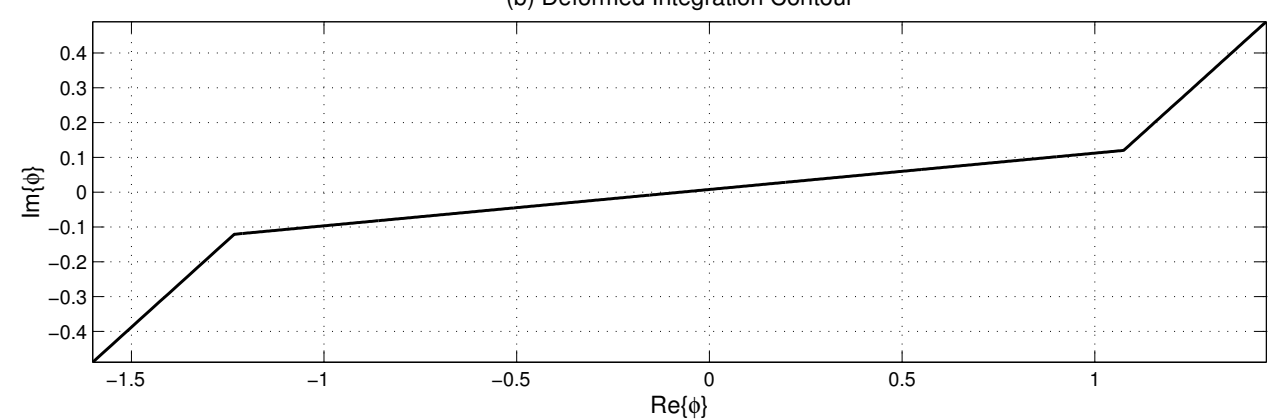

Figure 3.23: Deformed contour for a $500 \lambda$ terrain profile

(a) PEC Rough Surface Profile

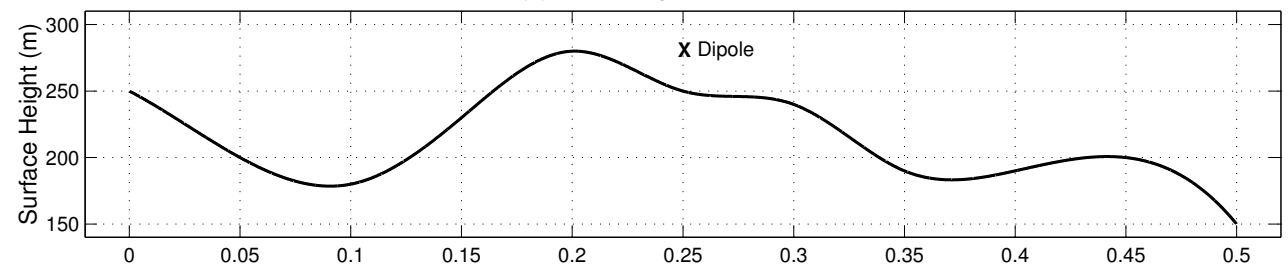

(b) TM Polarized Dipole on PEC Surface
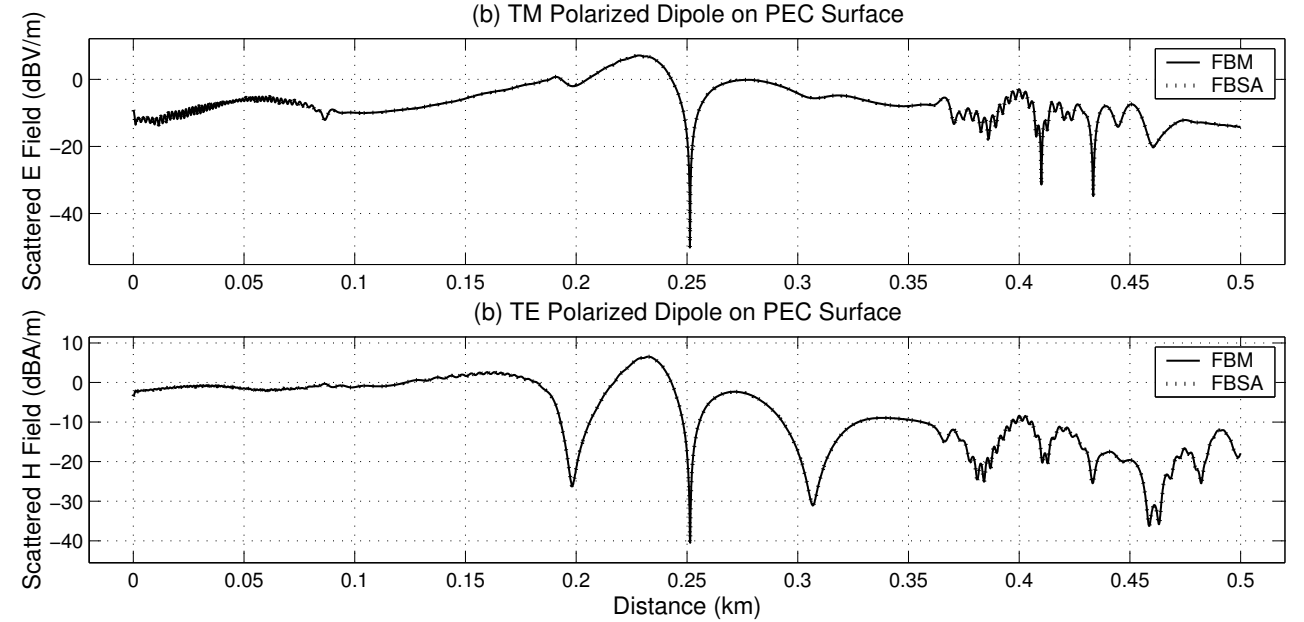

Figure 3.24: Scattered field from a $500 \lambda$ width terrain 
(a) non-PEC Rough Surface Profile

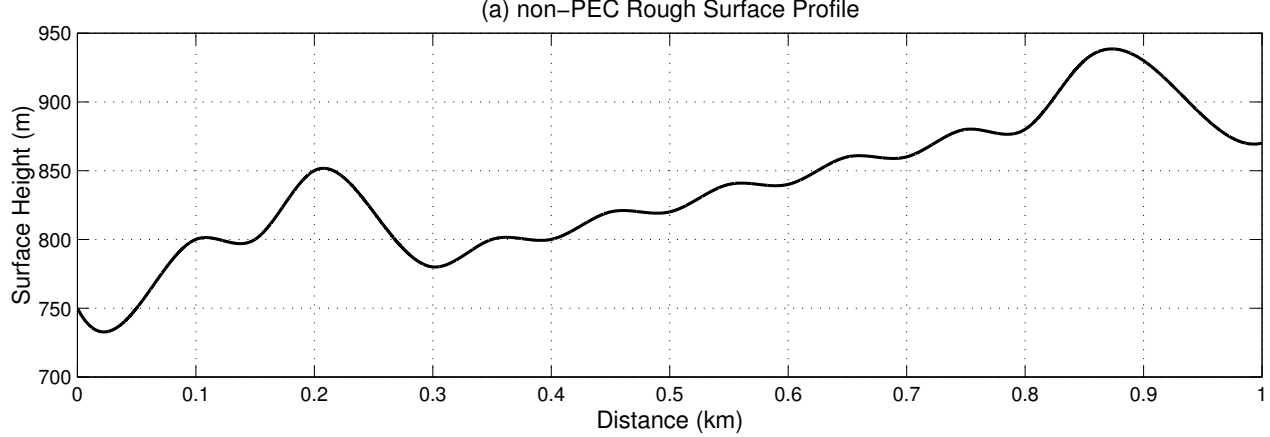

(b) Deformed Integration Contour

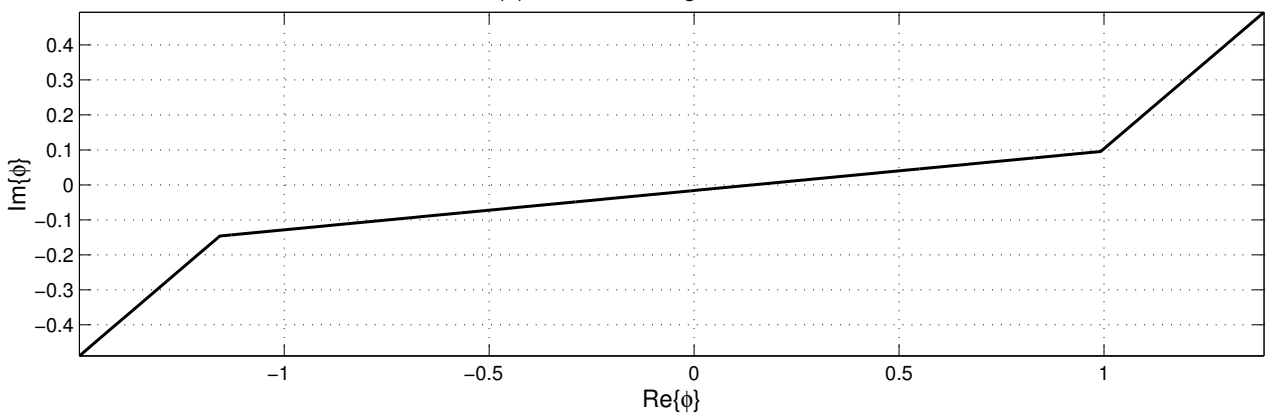

Figure 3.25: Deformed contour for a $1000 \lambda$ terrain profile

(a) non-PEC Terrain Profile

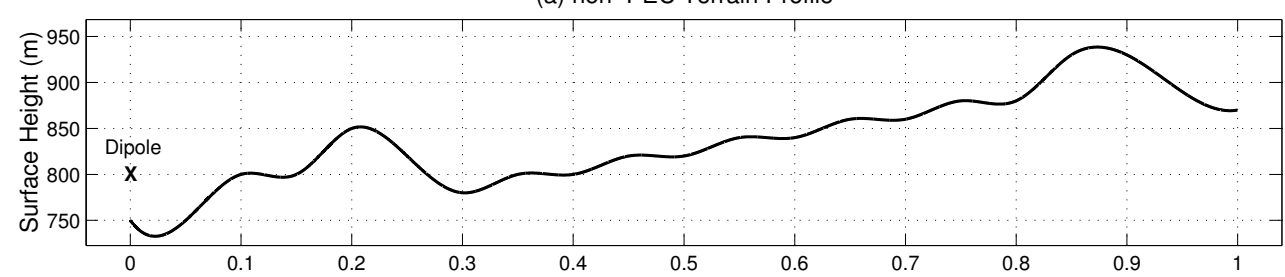

(b) TM Polarized Dipole on non-PEC Surface

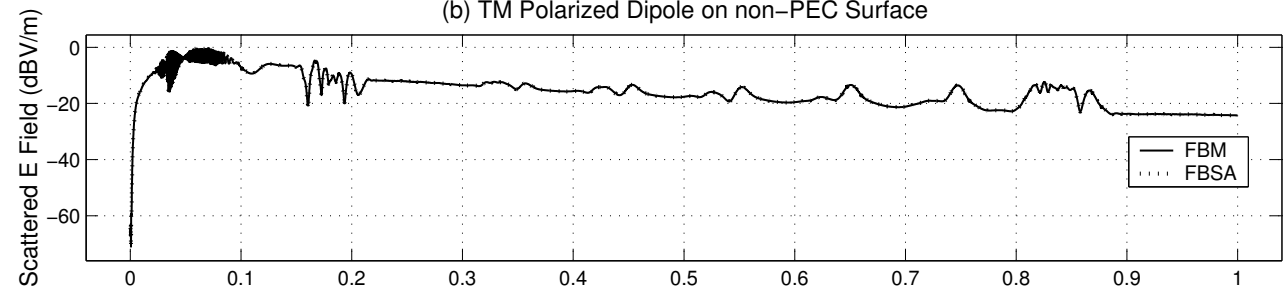

(b) TE Polarized Dipole on non-PEC Surface

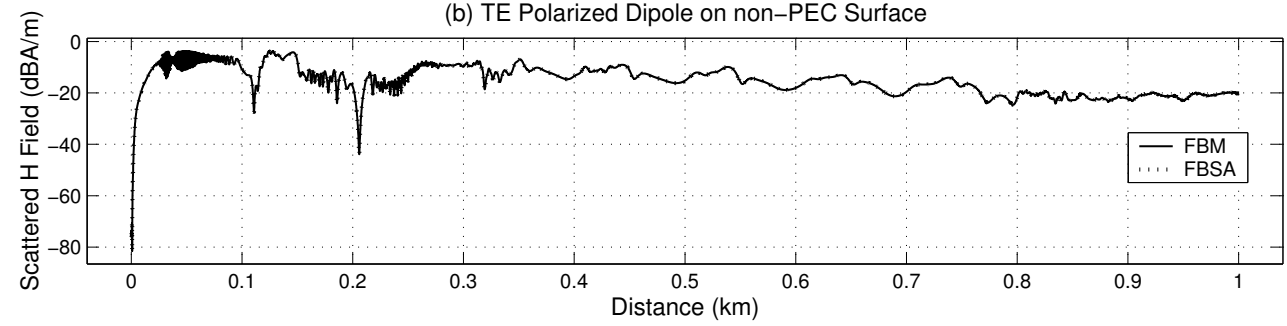

Figure 3.26: Scattered field from a $1000 \lambda$ width terrain 

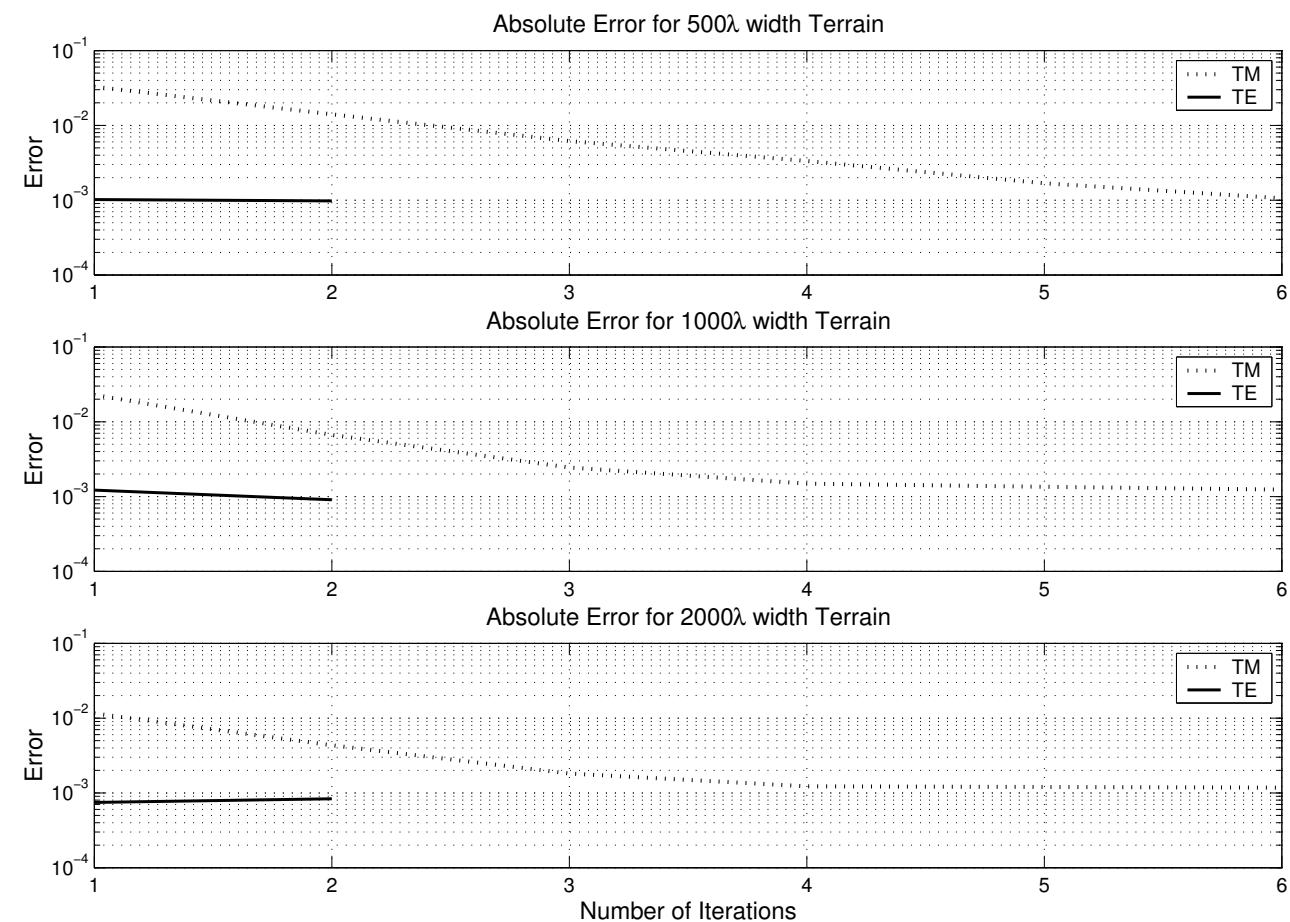

Figure 3.27: Absolute error for terrain profiles - FBSA vs FBM

This surface is considered to be illuminated by a dipole antenna located at a $40 \lambda$ height above the left-most point of the terrain as illustrated in Figure 3.25 (a). (3.41)-(3.43) and (3.45)-(3.47) are valid for computing the elements of $V_{n}$ for the horizontal and vertical polarization cases, respectively. $P_{t}$, the transmitted power from the infinitesimal dipole, is considered to be 40 Watts.

Figure 3.26 (b) and (c) illustrate the comparison of scattered fields, using FBM and FBSA on $1000 \lambda$ width terrain profile illustrated in Figure 3.26 (a) for TM and TE polarized infinitesimal dipole cases, respectively. The absolute error versus the number of iteration graphs, illustrated in Figure 3.27, show the accuracy of the method. Figure 3.28 (a) shows a $2000 \lambda$ width non-PEC $\left(\eta_{s}=20+j 20 \Omega\right)$ terrain profile. The study parameters of FBSA for this profile are included in Table 3.2 and the deformed contour of integration is illustrated in Figure 3.28 (b). Since the geometry is a downhill profile, the medium saddle point is located on the negative real axis. 


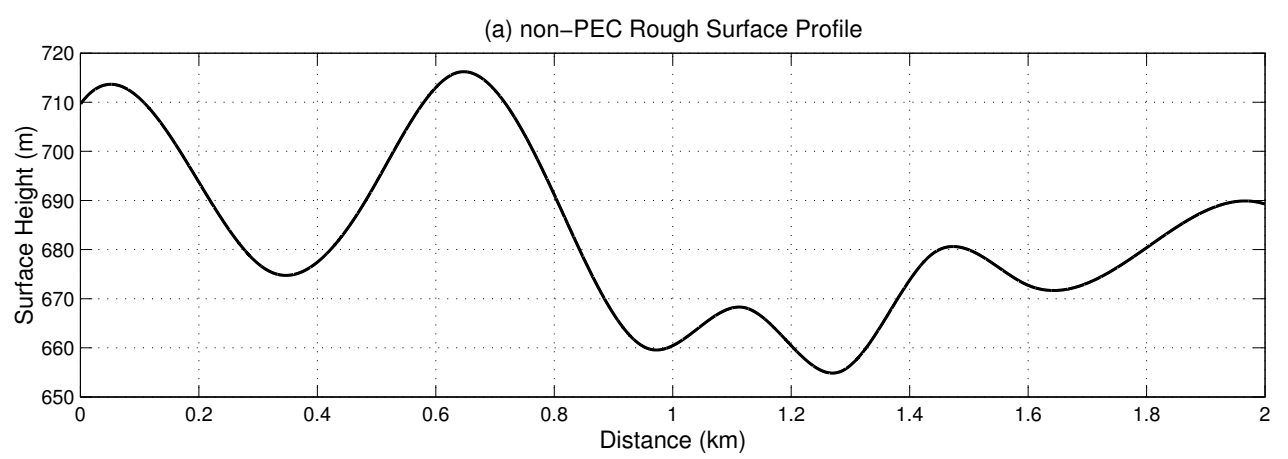

(b) Deformed Integration Contour

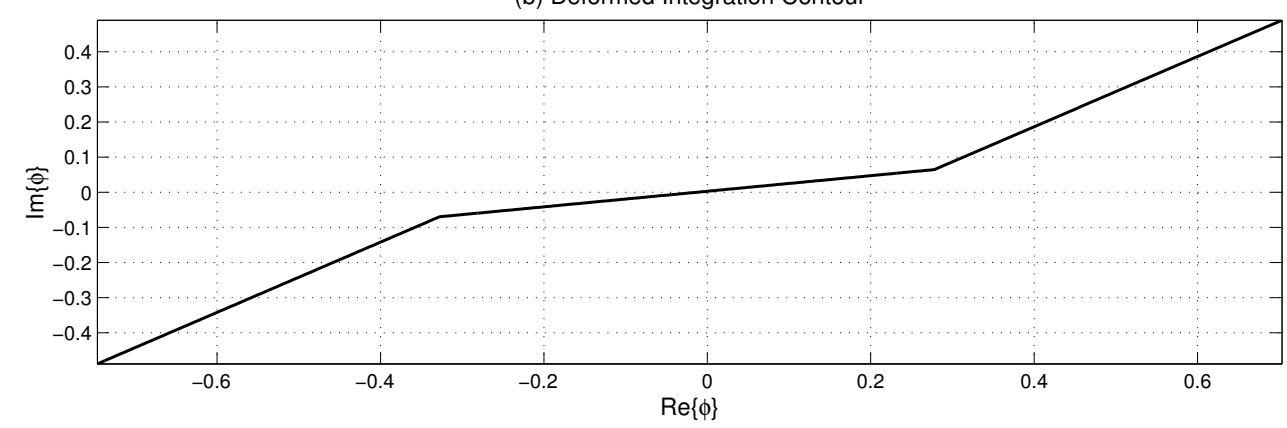

Figure 3.28: Deformed contour for a $2000 \lambda$ terrain profile
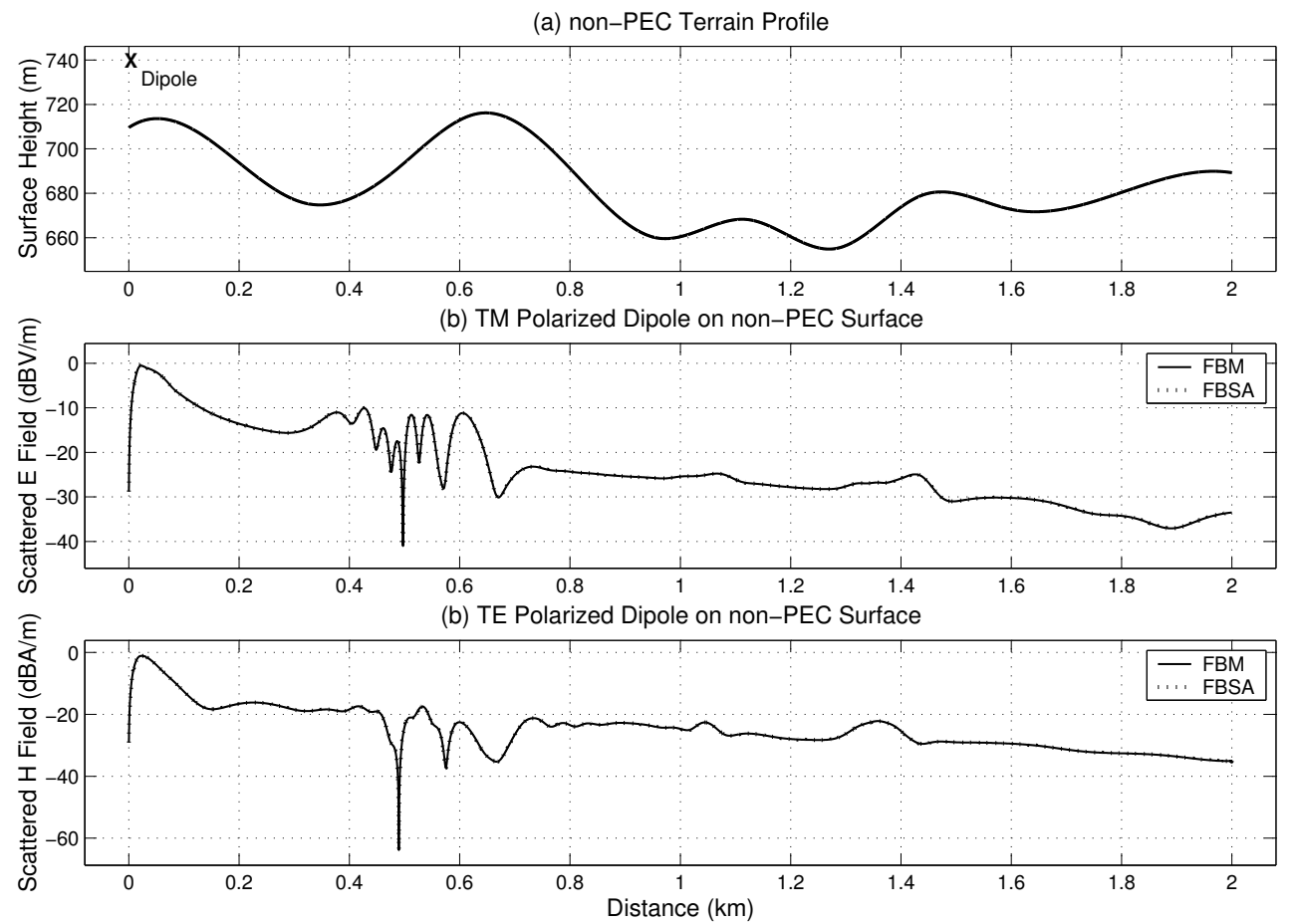

Figure 3.29: Scattered field from a $2000 \lambda$ width terrain 
This surface is considered to be illuminated by a dipole antenna located at a $25 \lambda$ height above the left-most point of the terrain. (3.41)-(3.43) and (3.45)(3.47) are valid for computing the elements of $V_{n}$ for the horizontal and vertical polarization cases, respectively. $P_{t}$, the transmitted power from the infinitesimal dipole, is considered to be 25 Watts.

Figure 3.29 (b) and (c) illustrate the comparison of scattered fields, using FBM and FBSA on 2000 $\lambda$ width terrain profile for TM and TE polarized infinitesimal dipole cases, respectively. The absolute error versus the number of iteration graphs are illustrated in Figure 3.27.

Obtaining the FBM reference solution becomes a cumbersome problem because of the $O\left(N^{2}\right)$ computational cost, after 20000 surface unknowns. However, tests of the FBSA shows that the FBSA solution can be used as a numerically accurate reference for computing the scattering from terrain profiles. Consider $5000 \lambda$ width non-PEC $\left(\eta_{s}=20+j 20 \Omega\right)$ terrain profile in Figure 3.30 (a). The parameters that are needed to implement the FBSA to this geometry are included in Table 3.3 and the deformed contour of integration is illustrated in Figure 3.30 (b). Since the geometry is a downhill profile, the medium saddle point is located on the negative real axis.

\begin{tabular}{|c|c|c|c|}
\hline Number of Unknowns $(N)$ & 50000 & 100000 & 200000 \\
\hline Frequency $(\mathrm{MHz})$ & 300 & 300 & 300 \\
\hline$\phi_{\text {med }}(\mathrm{rad})$ & -0.075 & 0.045 & -0.015 \\
\hline$\phi_{s, \min }(\mathrm{rad})$ & -0.488 & -0.138 & -0.230 \\
\hline$\phi_{s, \max }(\mathrm{rad})$ & 0.274 & 0.371 & 0.120 \\
\hline$L_{s}(\lambda)$ & 2 & 2 & 2 \\
\hline Num. of integr. points & 591 & 470 & 745 \\
\hline$\delta(\mathrm{rad})$ & 0.111 & 0.106 & 0.093 \\
\hline
\end{tabular}

Table 3.3: Study Parameters

This surface is considered to be illuminated by a dipole antenna located at a $60 \lambda$ height above the left-most point of the terrain. (3.41)-(3.43) are valid for computing the elements of $V_{n}$ for the horizontal polarization case. $P_{t}$, the 
transmitted power from the infinitesimal dipole, is considered to be 60 Watts. Figure 3.31 (b) illustrates the scattered field obtained using FBSA on 5000 $\lambda$ terrain profile for TM polarized infinitesimal dipole case.

Figure 3.32 (a) shows a $10000 \lambda$ width non-PEC $\left(\eta_{s}=20+j 20 \Omega\right)$ terrain profile. The study parameters of FBSA for this profile are included in Table 3.3 and the deformed contour of integration is illustrated in Figure 3.32 (b). Since the geometry is an uphill profile, the medium saddle point is located on the positive real axis.

This surface is considered to be illuminated by a dipole antenna located at a $60 \lambda$ height above the middle of the terrain. (3.41)-(3.43) and (3.45)-(3.47) are valid for computing the elements of $V_{n}$ for the horizontal and vertical polarization cases, respectively. $P_{t}$, the transmitted power from the infinitesimal dipole, is considered to be 90 Watts. Figure 3.33 (b) and (c) illustrate the scattered fields computed using FBSA on $10000 \lambda$ terrain profile for TM and TE polarized infinitesimal dipole cases, respectively.

Consider $20000 \lambda$ width non-PEC $\left(\eta_{s}=25+j 20 \Omega\right)$ terrain profile in Figure 3.34 (a). The parameters that are needed to implement the FBSA to this geometry are included in Table 3.3 and the deformed contour of integration is illustrated in Figure 3.34 (b). Since the geometry is a downhill profile, the medium saddle point is located on the negative real axis.

This surface is considered to be illuminated by a dipole antenna located at a $90 \lambda$ height above the left-most point of the terrain. (3.41)-(3.43) and (3.45)(3.47) are valid for computing the elements of $V_{n}$ for the horizontal and vertical polarization cases, respectively. $P_{t}$, the transmitted power from the infinitesimal dipole, is considered to be 90 Watts. Figure 3.35 (b) and (c) illustrate the scattered fields computed using FBSA on 20000 $\lambda$ terrain profile for TM and TE polarized infinitesimal dipole cases,respectively. 
(a) non-PEC Rough Surface Profile

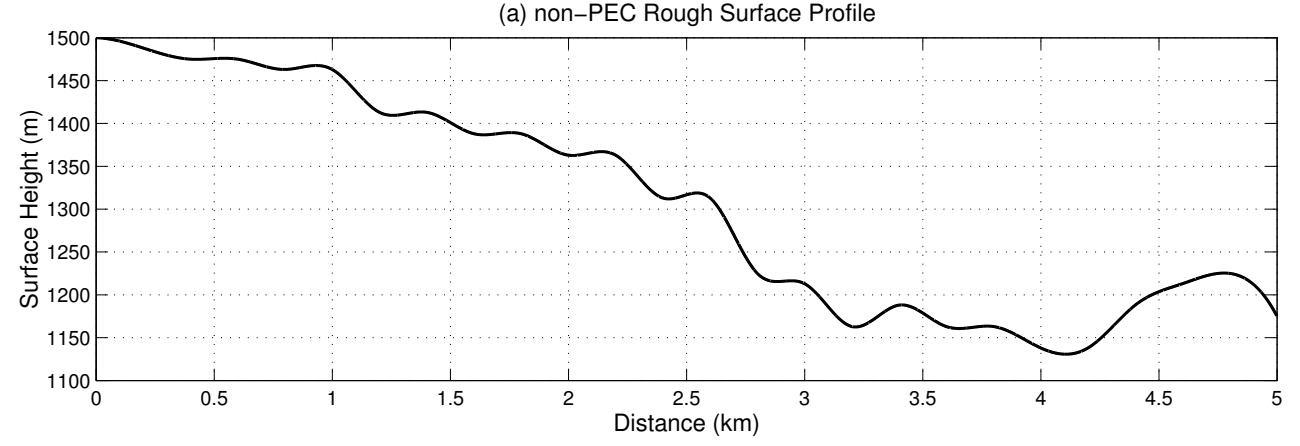

(b) Deformed Integration Contour

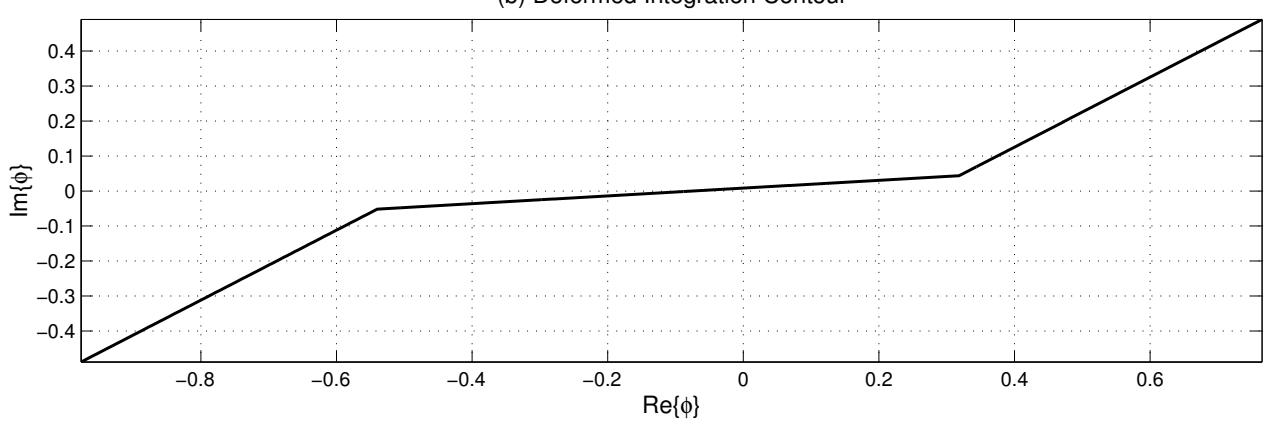

Figure 3.30: Deformed contour for a $5000 \lambda$ terrain profile

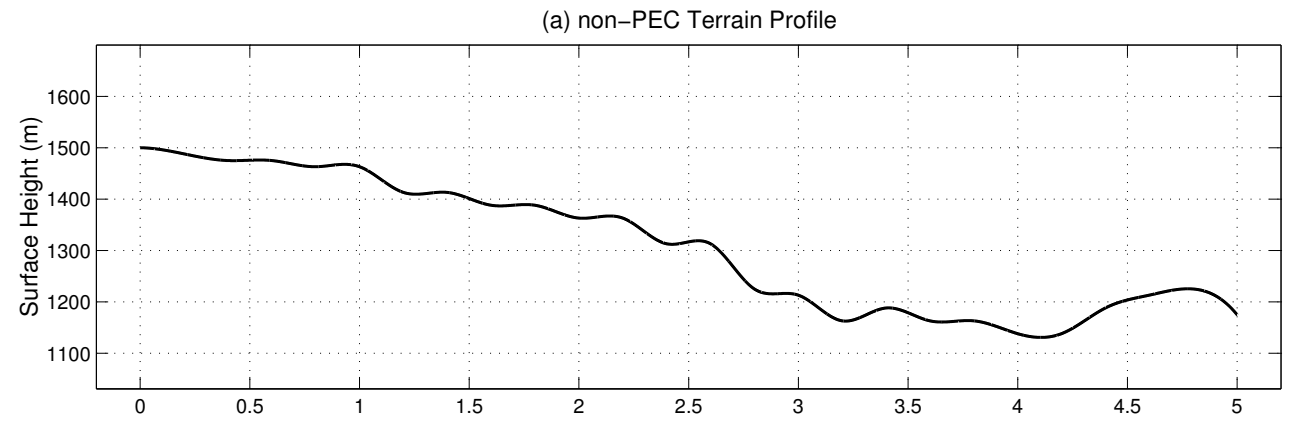

(b) TM Polarized Dipole on non-PEC Surface

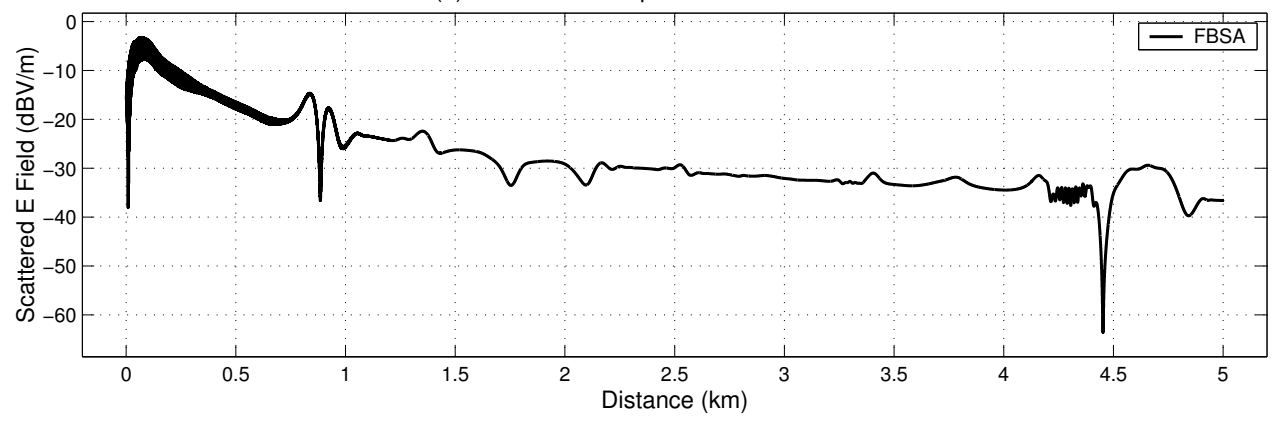

Figure 3.31: Scattered field from a $5000 \lambda$ width terrain 
(a) PEC Rough Surface Profile

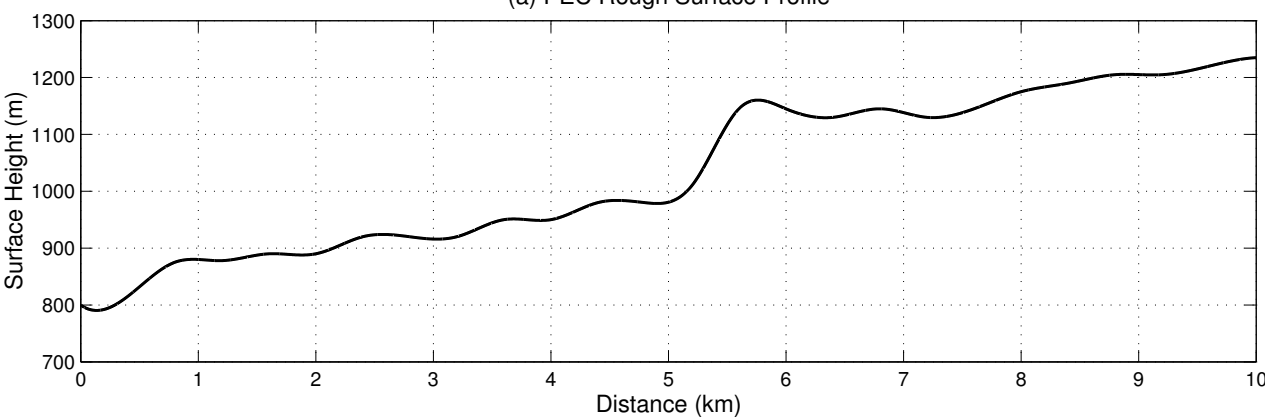

(b) Deformed Integration Contour

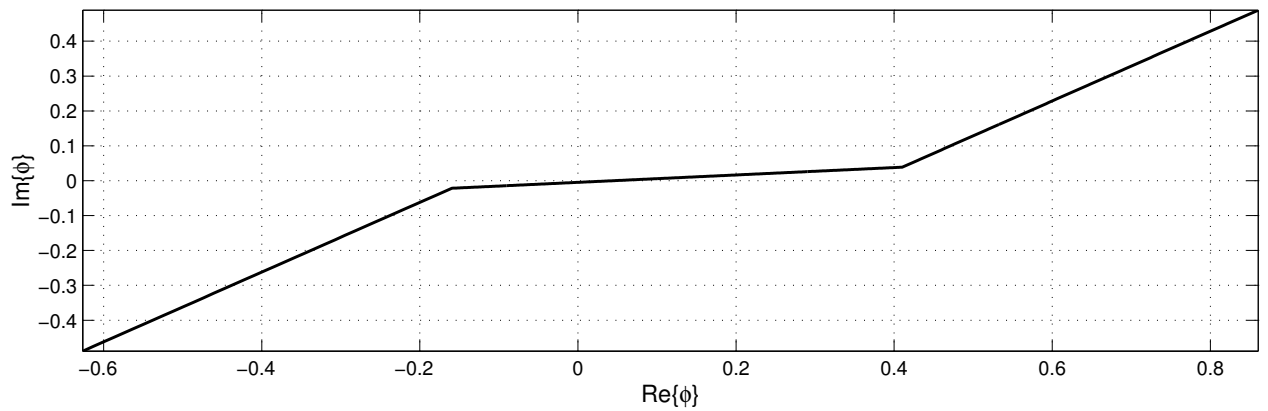

Figure 3.32: Deformed contour for a $10000 \lambda$ terrain profile

(a) PEC Terrain Profile

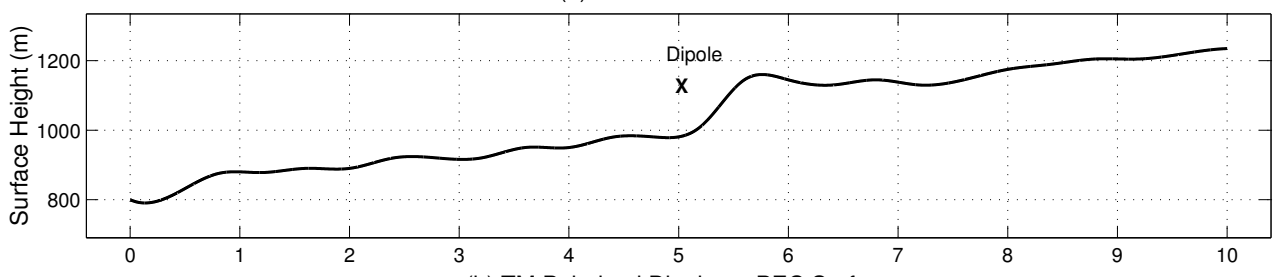

(b) TM Polarized Dipole on PEC Surface
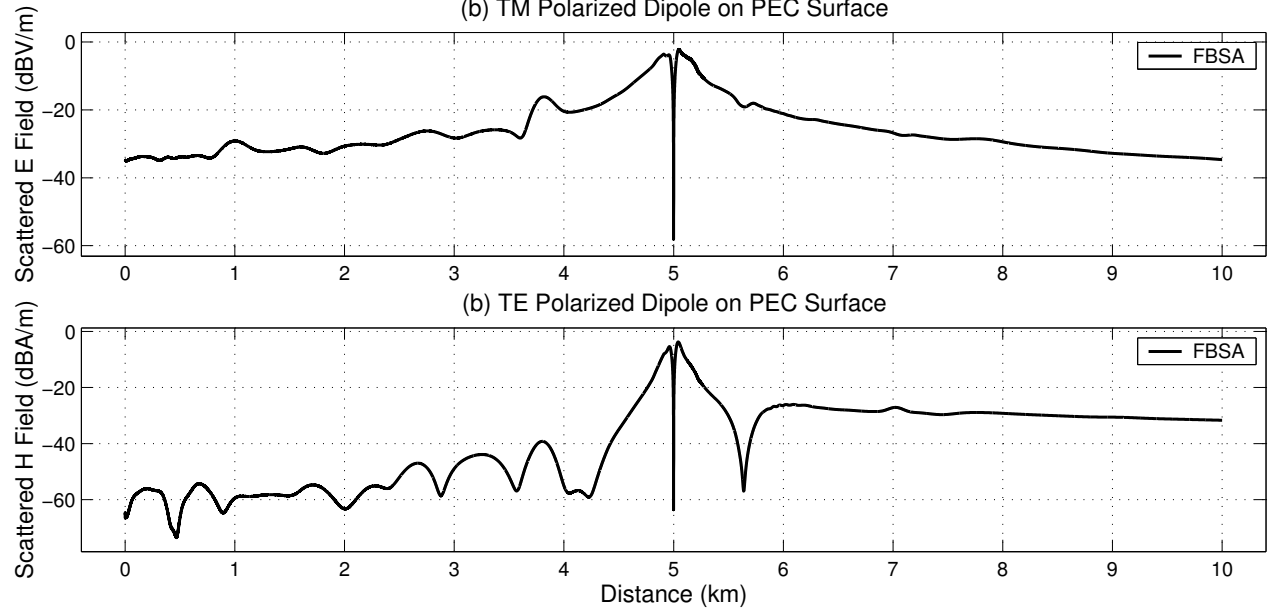

Figure 3.33: Scattered field from a 10000 $\lambda$ width terrain 


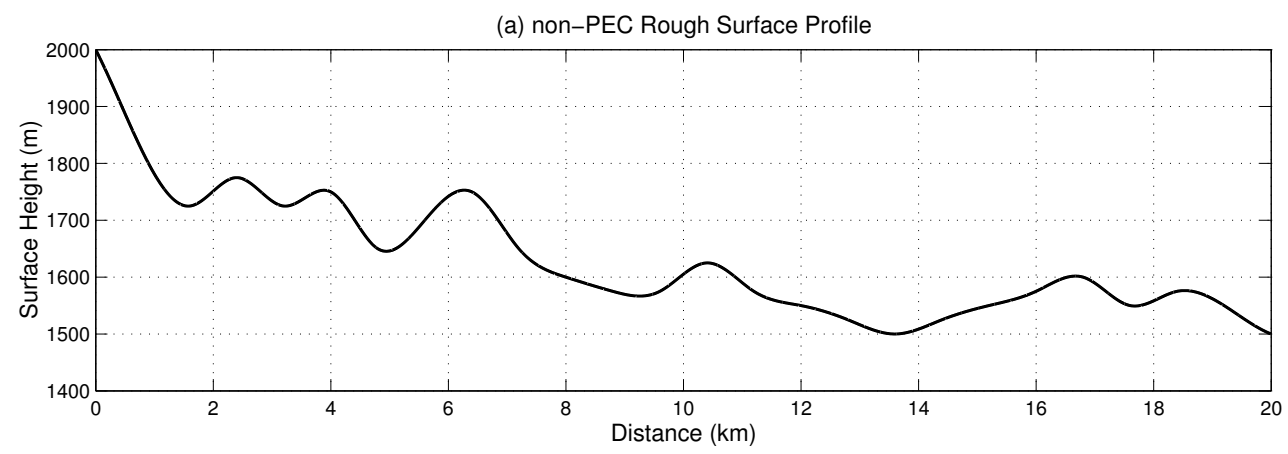

(b) Deformed Integration Contour

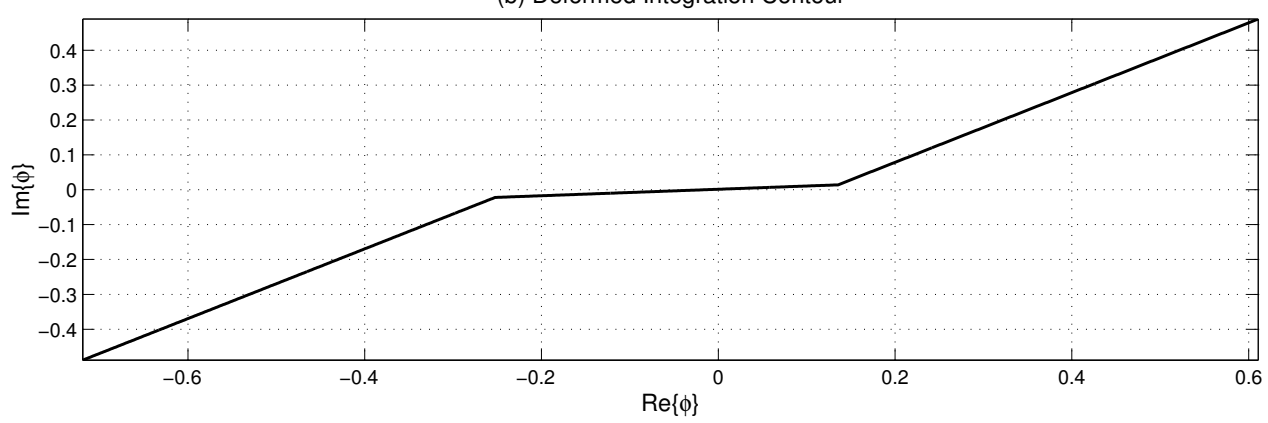

Figure 3.34: Deformed contour for a $20000 \lambda$ terrain profile
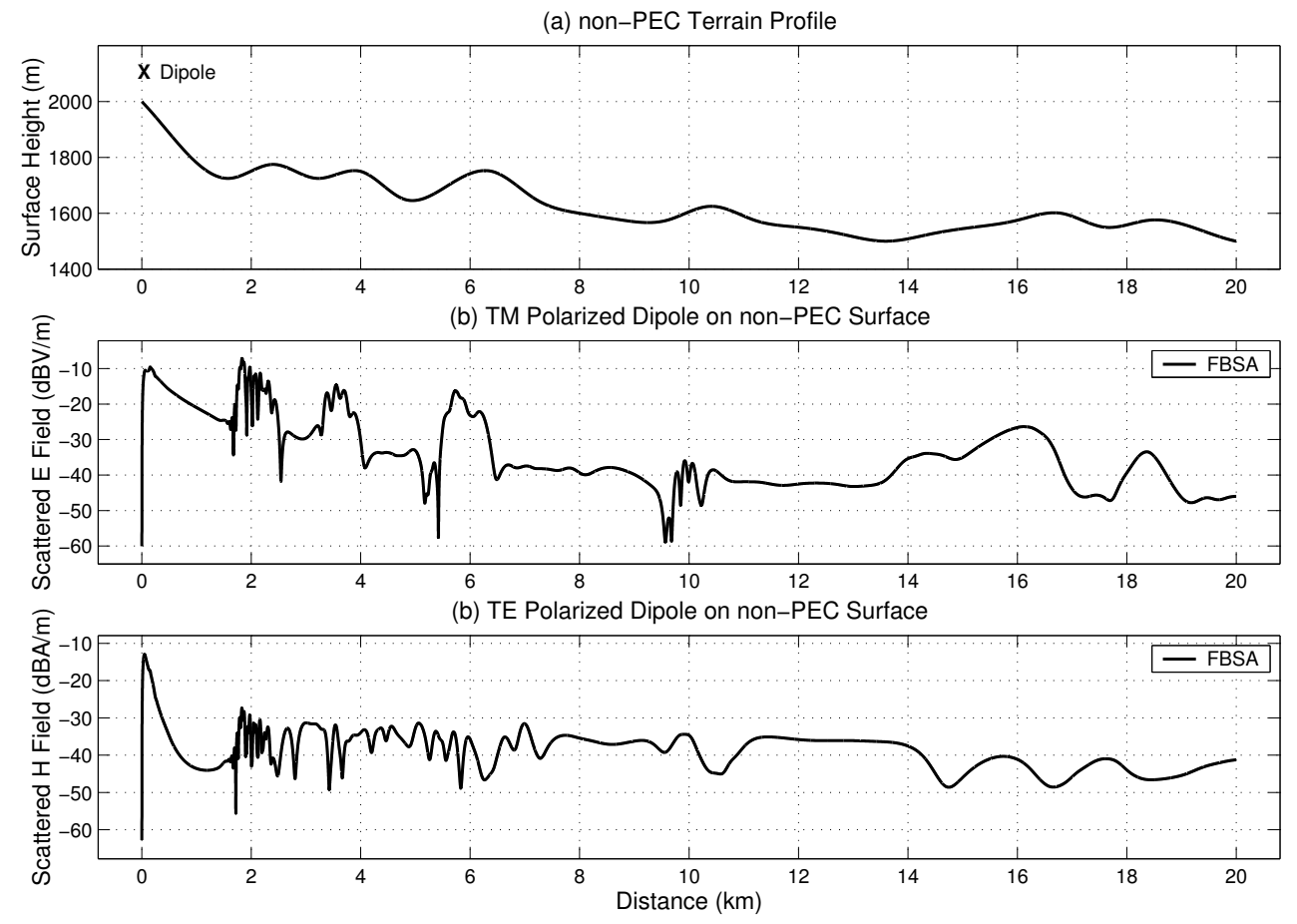

Figure 3.35: Scattered field from a 20000 $\lambda$ width terrain 


\begin{tabular}{|c|c|c|}
\hline Number of Unknowns $(N)$ & Num. of integr. points & CPU-time/iter (s) \\
\hline 2000 & 315 & 3.76 \\
\hline 5000 & 715 & 20.25 \\
\hline 10000 & 812 & 46.41 \\
\hline 20000 & 300 & 37 \\
\hline 50000 & 591 & 165.91 \\
\hline 100000 & 470 & 259.83 \\
\hline 200000 & 745 & 830.91 \\
\hline
\end{tabular}

Table 3.4: Computational Cost for the FBSA

For examination of the computational cost, Table 3.4 is given. The CPU-time values are obtained for the studies of the method on a Intel PIII microprocessor having a $500 \mathrm{MHz}$ clock frequency. CPU-time results show that the computational cost is very dependent on the number of integration points as the strong region distance stays constant. However, it is obvious that increasing the number of surface unknowns for a fixed number of integral steps, the modified algorithm yields an $O(N)$ computational time, comparing the cases of $N=2000$ with $N=20000$ and $N=5000$ case with $N=200000$.

The comparisons demonstrate that, the FBSA can be used as a numerically accurate reference solution instead of MoM or FBM over terrain profiles in both horizontal and vertical polarization cases, because of its very accurate results within very few iterations, causing a computational time and a memory requirement of $O(N)$.

\section{The Limitations of the Spectral Acceleration Algorithm for}

\section{Terrain Profiles}

A great number of numerical tests on the FBSA over terrain profiles show that, the algorithm is very good at computing the scattered field for inclination angle values larger than nearly 4 degrees. For more complex terrain profiles, the inclination angle of the deformed contour of integration becomes so small that the deformed contour of integration approaches close to both the real axis and 
its intersection with the SAP of the outermost saddle point. This point has a significant effect on the exponential growth of the integrand on the complex space. This also means that, the integrand will present very fast oscillations making very difficult to integrate it on the deformed contour. Therefore, the weak region contribution may suddenly increase exponentially and this may prevent the convergence of the method. In this case, it is possible to use different integration paths along the terrain profile. Then, the complete geometry will be divided in several portions each of which can be analyzed using a different inclination angle. After analyzing each portion of the geometry, a new integration path can be determined to analyze the rest of the terrain. This division process could be repeated several times along the terrain depending on the geometry. The use of different paths to analyze the whole terrain profile implies to recompute the integrand for each new integration path, but it is done only few times so the total cost is still $O(N)$. 


\section{Chapter 4}

\section{COMPARISONS OF}

\section{PROPAGATION MODELS}

\section{WITH FBSA SOLUTIONS}

Numerically accurate solution methods, like MoM, become not suitable for electrically large surfaces, because of the computational limitations due to the large number of surface unknowns. Because of such challenges, the development of automatic tools for radio coverage prediction over geographical data is a growing interest area. Therefore, the coverage and propagation loss study for wireless communications has become a focus of interest and a great number of propagation models have been developed. According to their nature, the propagation models can be classified as empirical, semi-empirical (or semi-deterministic), and deterministic models.

For the practical application of propagation models, there is an important tradeoff between the accuracy of the prediction and the speed with which the prediction can be made. Inserting semi-deterministic reflection and multiple diffraction (RMD) corrections into empirical prediction models may yield more 
accurate results than the empirical results. Also, they are relatively easy and fast to apply. Therefore, empirical models with RMD corrections are commonly used to predict the field strengths over terrain profiles.

All approaches to the radio propagation prediction present a similar behavior. However, these solution methods are obtained by a combination of guesswork and analysis so that they can not give clear physical results of the propagation process. So, choosing the best prediction model among a great number of methods becomes an important problem. In this regard, numerically accurate propagation models, such as integral equation based methods, become very desirable because they would avoid any kind of uncertainty in the electromagnetic analysis and, therefore, could be used to check the sensitivity of the true solution to the input terrain data. Besides, they could be used as a reference solution as an alternative to measurements to validate and clarify the limitations of other heuristic and intuitive methods involving approximations.

In this chapter, empirical propagation prediction models with reflection and multiple diffraction (RMD) corrections are examined. The most common empirical models and multiple diffraction correction methods are explained in Sections 4.1 and 4.2, respectively. Comparisons with the numerically accurate solutions using FBSA are given in Section 4.3, to find out the best propagation models, finally.

\subsection{Empirical Propagation Models}

Empirical models are described by equations or curves derived from statistical analysis of a large number of measured data. Among the empirical methods for predicting the field strength and path loss over terrain profiles for VHF-UHF frequencies, International Telecommunication Union Recommendations [8]-[10], 
Federal Communications Commission curves [11] and Okumura-Hata [43]-[44] are considered to be the most significant ones.

\subsubsection{The Okumura-Hata Model}

The original Hata model was published in 1980 by Masaharu Hata [44]. Hata took the information for the field strength curves produced by Yoshihisa Okumura [43] and produced a set of equations for path loss. Okumura carried out a number of propagation studies in and around Tokyo City and produced a set of curves of field strength against distance.

Two of the limitations of the Hata model are that it has a limited path length and a limited frequency range. A number of modified models have been produced to extend the path length and frequency range. These modified models vary slightly from each other and some of these models more closely match the Okumura curves than others do.

The Hata empirical model uses a propagation equation split up into two terms, a term that has a logarithmic dependence on distance and a term that is independent of distance. The Hata model also includes adjustments to the basic equation to account for urban, suburban and open area propagation losses. The Hata equation for propagation loss in an urban area is given by:

$$
L_{p}=69.55+26.16 \log f-13.82 \log h_{b}-a\left(h_{m}\right)+\left(44.9-6.55 \log h_{b}\right) \log d
$$

in $\mathrm{dB}$, where $f$ denotes the operating frequency in $\mathrm{MHz}, h_{b}$ is the height of the base station or transmitter in meters, $h_{m}$ the height of the mobile or receiver in meters, $d$ is the distance between the receiver and transmitter in kilometers, and

$$
a\left(h_{m}\right)=(1.1 \log f-0.7) h_{m}-(1.56 \log f-0.8) .
$$

The adjustment for propagation loss in an open area is:

$$
L_{p o}=L_{p}-4.78[\log f]^{2}+18.33 \log f-40.94 \text {. }
$$


The model is suitable for use over the ranges:

- Frequency range 150 - $1500 \mathrm{MHz}$

- Base station height $30-200 \mathrm{~m}$

- Mobile height 1 - $10 \mathrm{~m}$

- Distance range 1 - $20 \mathrm{~km}$

The modified Hata models were produced to improve on the range limitation that the original Hata model had. The first modified model appeared in a CCIR report in an attempt to extend the Hata model to cover greater distance. Later on an ITU-R recommendation, ITU-R P.529-3, is presented as a modified Hata model in [9].

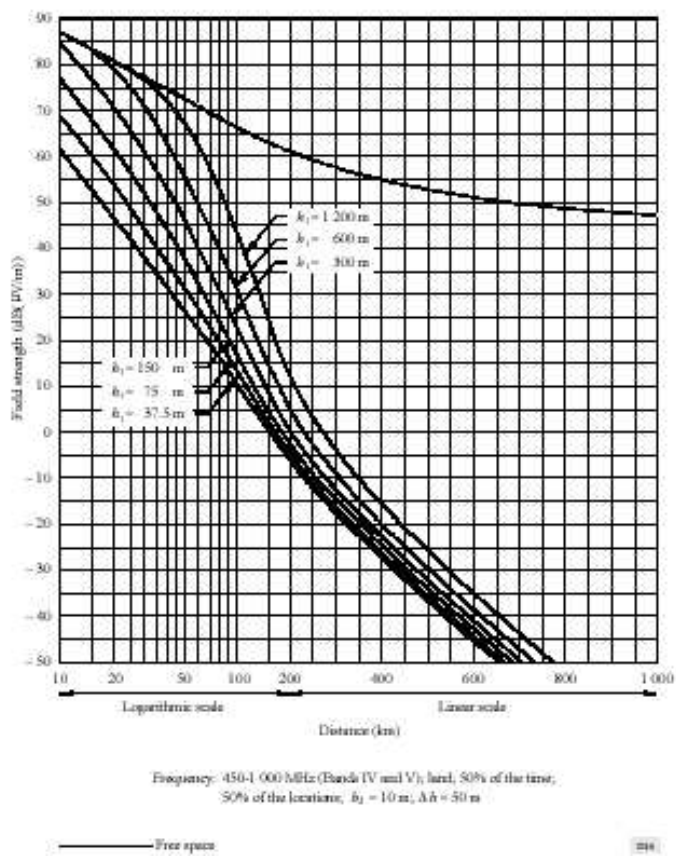

Figure 4.1: ITU-R Rec.370 Curves. 


\subsubsection{International Telecommunication Union Recom- mendations}

Recommendation ITU-R P.370-7 The ITU Radiocommunication Bureau recommends the curves such as given in Figure 4.1, to be adopted for the prediction of field strength for the broadcasting service for the frequency range 30 to $1000 \mathrm{MHz}$ and for the distance range up to $1000 \mathrm{~km}$, considering that there is a need to give guidance to engineers in the planning of broadcast services in the VHF and UHF bands for all climatic conditions in [8]. The curves are based on the statistical analysis of a considerable amount of experimental data.

Recommendation ITU-R PN.525-2 (Free Space Propagation Model) The ITU Radiocommunication Bureau recommends the calculation of attenuation in free space considering free-space propagation is a fundamental reference for radio-engineering in [45].

If there is a transmitter serving several randomly-distributed receivers (broadcasting, mobile service), the field is calculated at a point located at some appropriate distance from the transmitter by the expression:

$$
e=\frac{\sqrt{30 p}}{d}
$$

where $e$ is the root mean square field strength in $\mathrm{V} / \mathrm{m}, p$ is the equivalent isotropically radiated power (EIRP) of the transmitter in the direction of the point in Watts and $d$ is the distance from the transmitter to the point in meters.

Recommendation ITU-R P.529-3 The ITU Radiocommunication Bureau recommends the curves such as given in Figure 4.2 to be used to provide guidance on the prediction of point-to-area field strength for the land mobile service in the VHF and UHF bands, considering there is a need to give guidance to engineers in the planning of land mobile radio services in the VHF and UHF bands in [9]. 
This recommendation provides curves for predicting field strength under average conditions for three frequency ranges. It also provides analytical expressions which are valid for certain frequency ranges and conditions, and various correction factors which can be used to refine the average predictions.

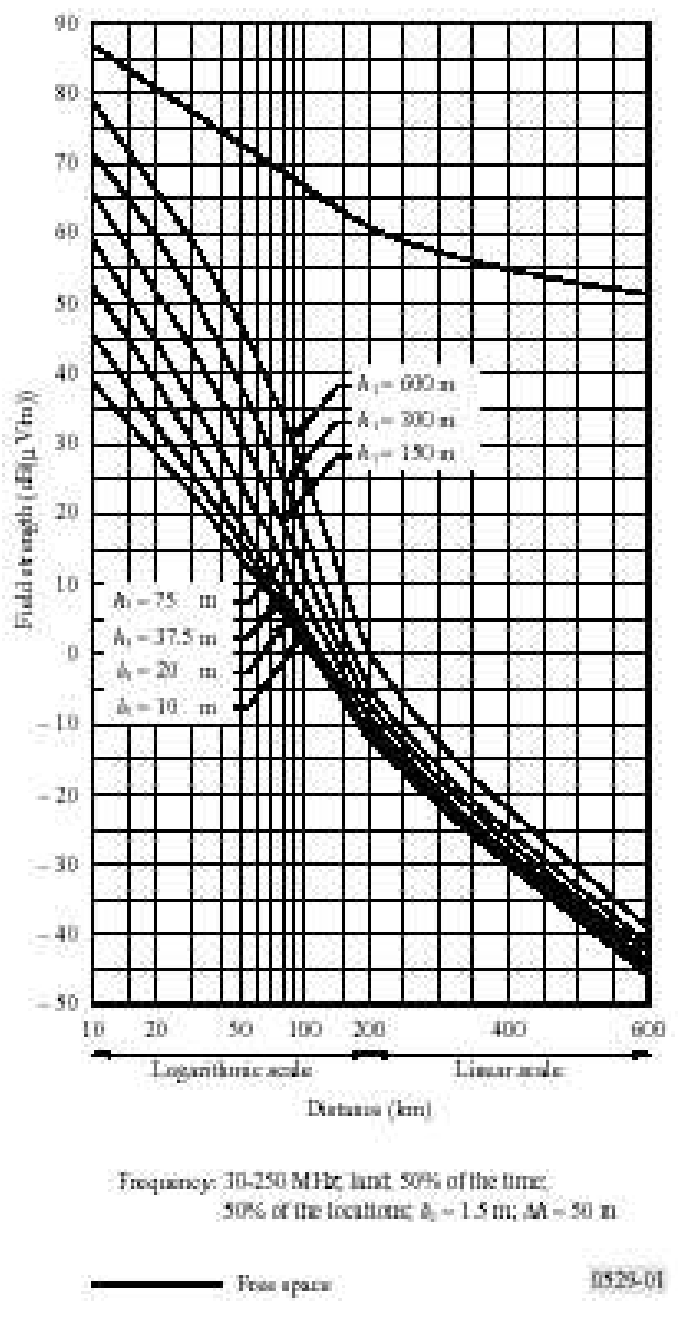

Figure 4.2: ITU-R Rec.529 Curves.

This recommendation proposes a modified Hata model. The equation in this model includes a modification to the base height term different from the original Hata. This equation does equate to the original Hata equation for distances less than $20 \mathrm{~km}$. The equation for the field strength at a distance from a $1 \mathrm{~kW}$ ERP transmitter in $\mathrm{dB} \mu \mathrm{V} / \mathrm{m}$ is: 


$$
E=69.82-6.16 \log f-13.82 \log h_{b}-a\left(h_{m}\right)+\left(44.9-6.55 \log h_{b}\right)[\log d]^{b}
$$

where $b$ is 1 if the distance between the transmitter and receiver is less than 20 kilometers. If $d>20$ kilometers, then

$$
b=1+\left(0.14+1.87 \times 10^{-4} \times f+1.07 \times 10^{-3} \times h_{b}^{\prime}\right)\left[\log \frac{d}{20}\right]^{0.8} .
$$

Here $h_{b}^{\prime}$ is given by:

$$
h_{b}^{\prime}=\frac{h_{b}}{\sqrt{1+7 \times 10^{-6} \times h_{b}^{2}}} .
$$

The model is suitable for use over the ranges:

- Frequency range 150 - $1500 \mathrm{MHz}$

- Base station height 30 - $200 \mathrm{~m}$

- Mobile height 1 - $10 \mathrm{~m}$

- Distance range 1 - $100 \mathrm{~km}$

Recommendation ITU-R P.1546 The ITU Radiocommunication Bureau recommends the curves such as given in Figure 4.3 to be adopted for pointto-area prediction of field strength for the broadcasting, land mobile, maritime mobile and certain fixed services (e.g. those employing point-to-multipoint systems) in the frequency range $30 \mathrm{MHz}$ to $3000 \mathrm{MHz}$ and for the distance range $1 \mathrm{~km}$ to $1000 \mathrm{~km}$, considering that there is a need to give guidance to engineers in the planning of terrestrial radiocommunication services in the VHF and UHF bands in [10]. The curves are based on the statistical analysis of experimental data.

This recommendation differs from others with using upper limits for field strengths and terrain clearance angle correction. Also this method takes the variability of atmospheric refractive index into account and gives equations for computing the land curves. 


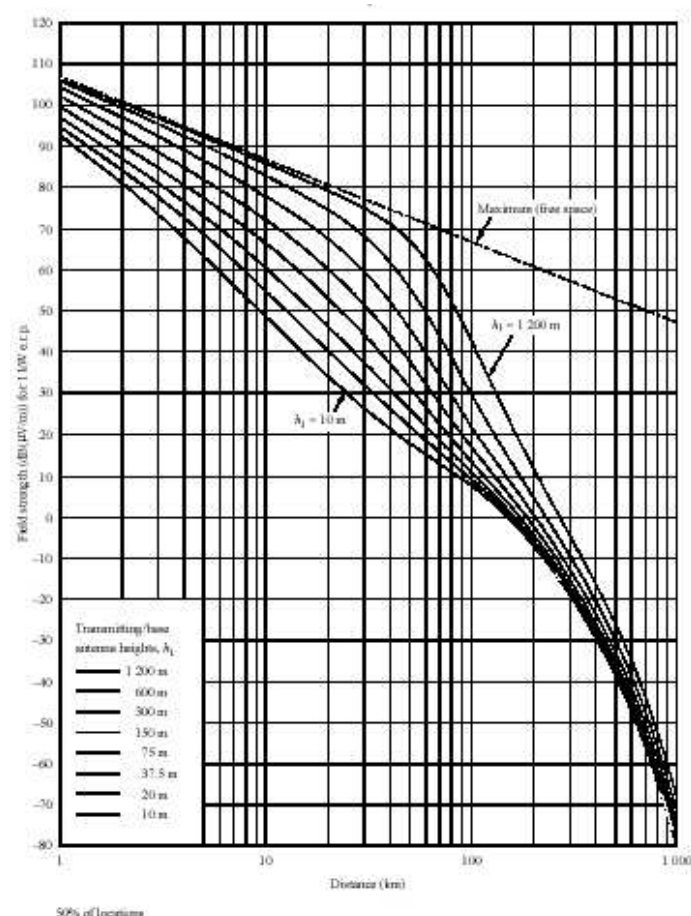

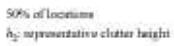

Figure 4.3: ITU-R Rec.1546 Curves.

\subsubsection{Federal Communications Commission Curves}

The Federal Communications Commission (FCC) is an independent United States government agency, directly responsible to Congress. The FCC was established by the Communications Act of 1934 and is charged with regulating interstate and international communications by radio, television, wire, satellite and cable. FCC recommends the curves such as depicted in Figure 4.4 for the prediction of the field strength for different frequency bands. The curves are based on the statistical analysis of experimental data.

Empirical models present similar behavior in predicting the field strength attenuation along terrain profiles, however, they are not able to detect the fluctuations in field strength due to the diffraction. Inserting semi-deterministic reflection and multiple diffraction (RMD) corrections into empirical prediction models may yield more accurate results than the empirical results. 


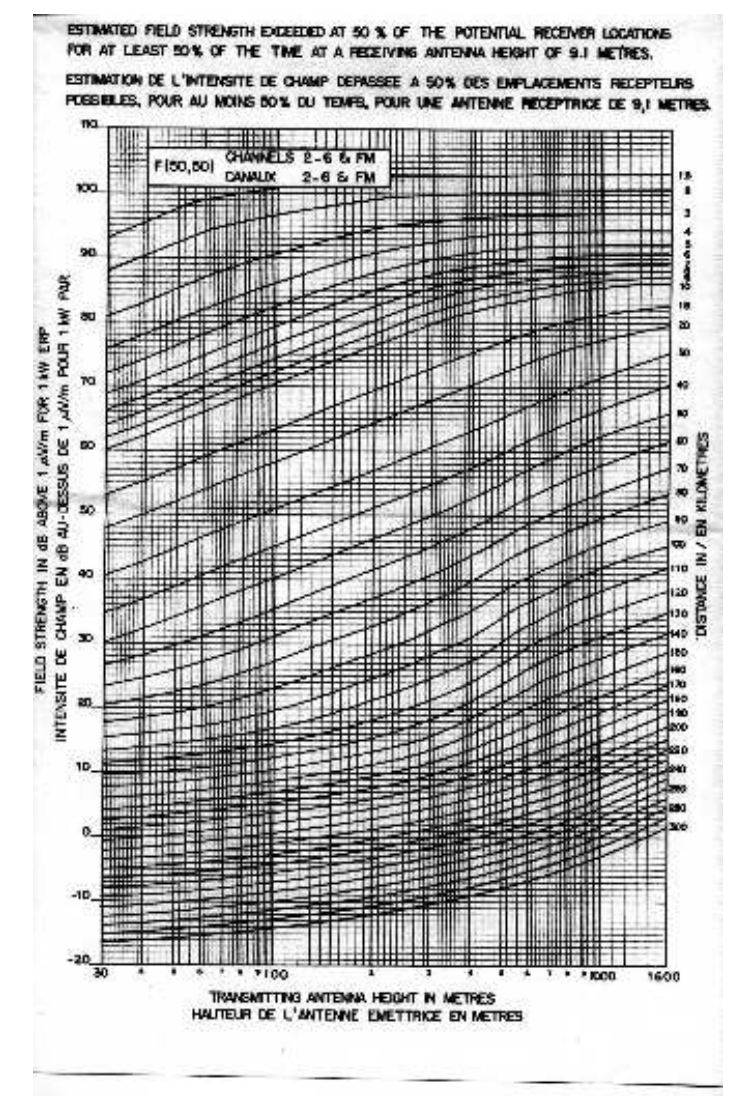

Figure 4.4: FCC Curves.

\subsection{Multiple Diffraction Corrections}

Many propagation paths encounter one obstacle or several separate obstacles and it is useful to estimate the losses caused by such obstacles. To make such calculations, it is necessary to idealize the form of the obstacles, either assuming a knife-edge of negligible thickness or a thick smooth obstacle with a well-defined radius of curvature at the top.

If the direct line-of-sight is obstructed by a single knife-edge type of obstacle as illustrated in Figure 4.5, with height $h_{m}$, the diffraction parameter $\nu$ is defined as:

$$
\nu=h_{m}\left[\sqrt{\frac{2}{\lambda}\left(\frac{1}{d_{t}}+\frac{1}{d_{r}}\right)}\right]
$$




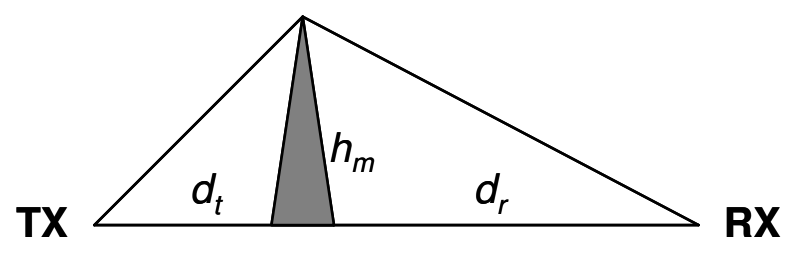

Figure 4.5: Path profile model for single knife edge diffraction.

where $d_{t}$ and $d_{r}$ are the terminal distances from the knife edge. The diffraction loss is expressed in $\mathrm{dB}$, and it can be closely approximated by

$$
A_{d}(d B)=\left\{\begin{array}{ll}
0 & , \nu<0 \\
6+9 \nu-1.27 \nu^{2} & , 0<\nu<2.4 \\
13+\log \nu & , 2.4<\nu
\end{array} .\right.
$$

The attenuation over rounded obstacles is usually higher than $A_{d}$ in the above formula. Detailed information about various diffraction loss methods is given in an ITU recommendation [46]. Approximate techniques to compute the diffraction loss over multiple knife edges have been also recommended by ITU.

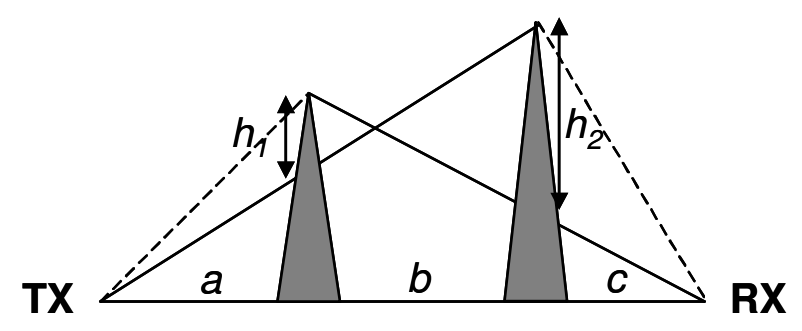

Figure 4.6: Epstein-Peterson method geometry.

The Epstein-Peterson method consists of applying single knife-edge diffraction theory successively to the two obstacles, with the top of the first obstacle acting as a source for diffraction over the second obstacle as illustrated in Figure 4.6. It has been observed that the Epstein-Peterson method yields good results, when the two obstacles are close to each other [47]. 


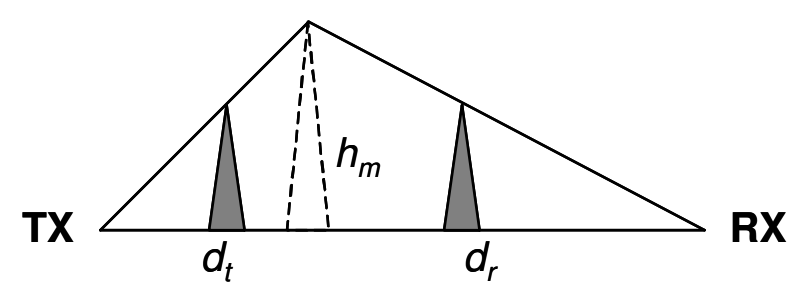

Figure 4.7: Bullington method geometry.

The method by Bullington defines a new 'effective' obstacle at the point where the line-of-sight from the two antennas cross [48]. Figure 4.7 shows the construction for approximate calculation of multiple knife-edge diffraction loss, proposed by Bullington.

Deygout suggested to search the 'main' obstacle, i.e., the point with the highest value of $\nu$ along the path. Diffraction losses over 'secondary' obstacles are added to the diffraction loss over the main obstacle [49]. Figure 4.8 shows the construction for approximate calculation of multiple knife-edge diffraction loss, proposed by Deygout.

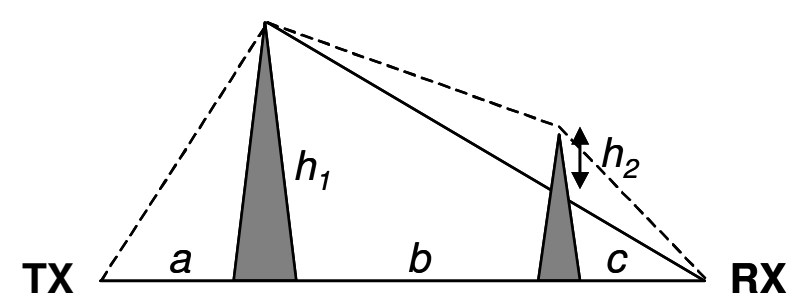

Figure 4.8: Deygout method geometry.

The empirical models recommended by ITU are simple and do not require detailed information about the environment. They are also easy and fast to apply because the estimation is usually obtained from closed expressions. However, they can not provide a very accurate estimation of the scattered field or the path loss. In the previous work, an important number of examinations of integral equation methods based on 2D Green's function with measurement data or Hata 
model have been presented [18], [20]. In this work, using the FBSA as reference solution, the most common propagation prediction models are examined.

\subsection{Numerical Results}

In this Section, the FBSA is used as a reference solution to compare the most common propagation models. The FBSA solutions over two different terrain profiles of $20 \mathrm{~km}$ width, one of which is a downhill profile and the other is an uphill one, are obtained for three different operating frequencies; for 200, 500 and finally for $890 \mathrm{MHz}$. The total field strengths are evaluated over a copy of each original terrain, $h=1.8$ meters above.

Firstly, the $200 \mathrm{MHz}$ case is examined. Figure 4.9 (a) and 4.10 (a) show the downhill (DH) and uphill (UH) terrain profiles, respectively, and Figure 4.9 (b) and 4.10 (b) illustrate their deformed integration paths for $200 \mathrm{MHz}$. Required parameters for the spectral acceleration are included in Table 4.1 for this case.

As expected, the medium saddle point is located on the negative real axis for the downhill profile and on the positive real axis for the uphill profile, even though they are very close to the terrain.

\begin{tabular}{|c|c|c|}
\hline & Downhill & Uphill \\
\hline Frequency $(\mathrm{MHz})$ & 200 & 200 \\
\hline$\lambda(\mathrm{m})$ & 1.5 & 1.5 \\
\hline width $(\lambda)$ & 13333.3 & 13333.3 \\
\hline$N$ & 133333 & 133333 \\
\hline$\phi_{\text {med }}(\mathrm{rad})$ & -0.003 & 0.011 \\
\hline$\phi_{s, \text { min }}(\mathrm{rad})$ & -0.336 & -0.233 \\
\hline$\phi_{s, \text { max }}(\mathrm{rad})$ & 0.169 & 0.209 \\
\hline$L_{s}(\lambda)$ & 3.333 & 3.333 \\
\hline Num. of integr. points & 225 & 286 \\
\hline$\delta(\mathrm{rad})$ & 0.273 & 0.174 \\
\hline
\end{tabular}

Table 4.1: Study Parameters for $200 \mathrm{MHz}$ 
(a) non-PEC Terrain Profile

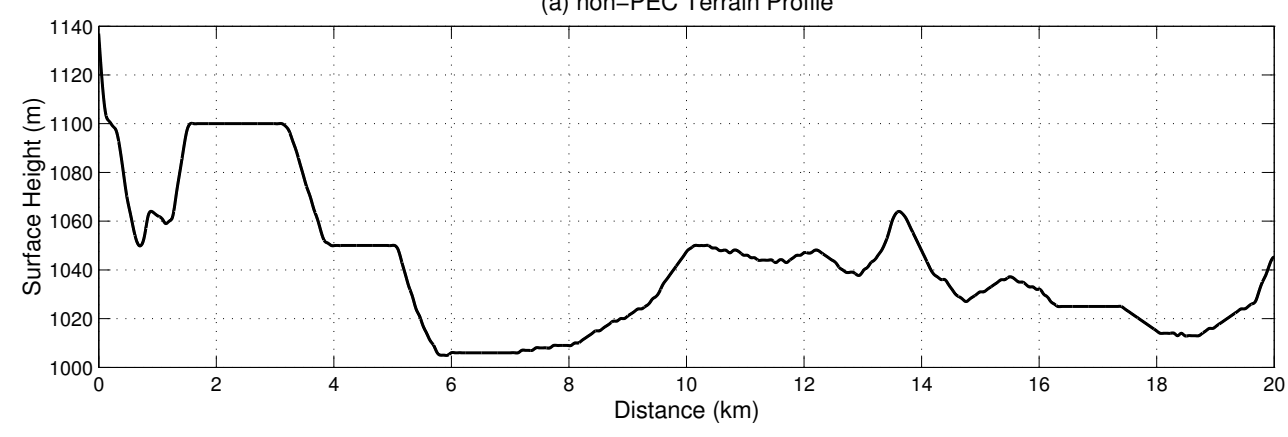

(b) Deformed Integration Contour

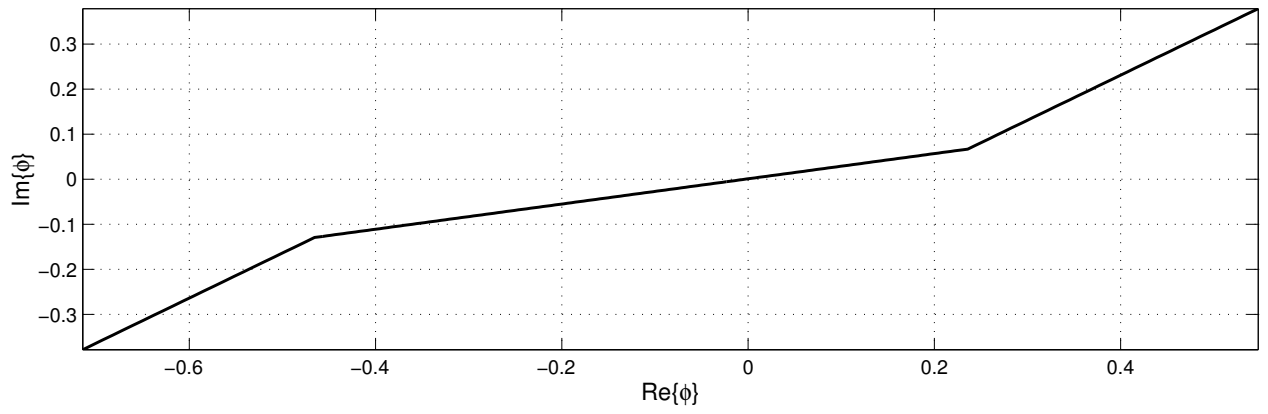

Figure 4.9: Downhill terrain profile and deformed path.

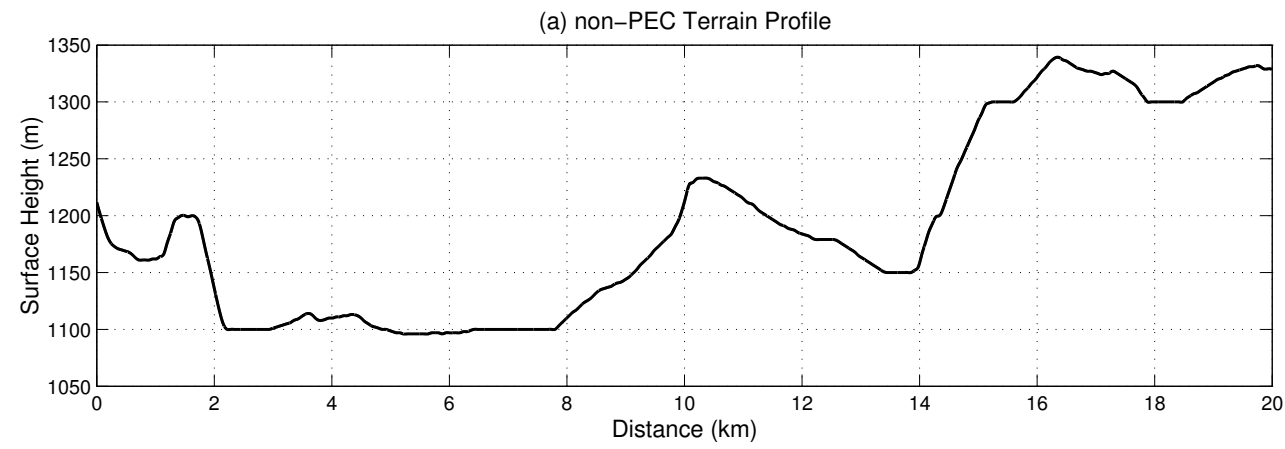

(b) Deformed Integration Contour

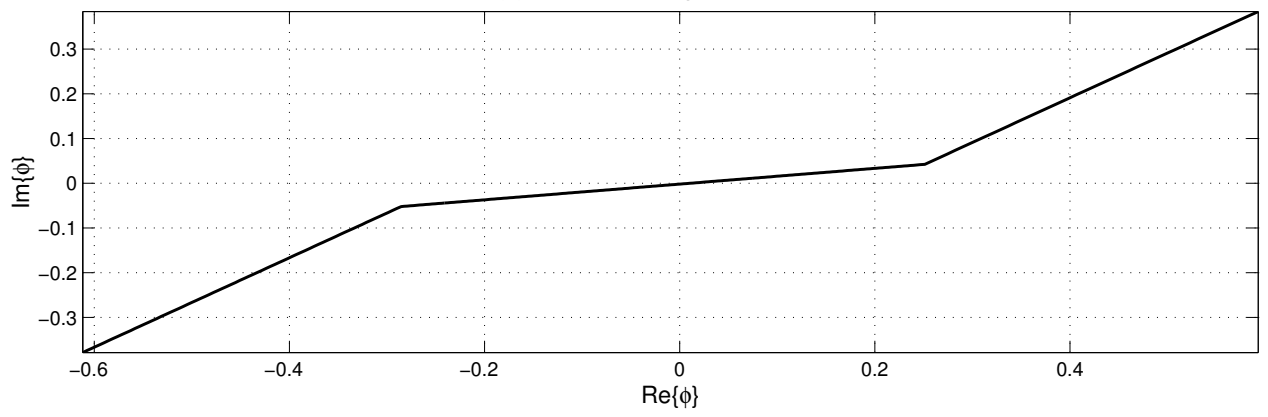

Figure 4.10: Uphill terrain profile and deformed path. 
Profiles are considered to be impedance surfaces of $\eta_{s}=25+j 20 \Omega$. At 200 $\mathrm{MHz}$, this impedance corresponds to a relative permittivity of $\epsilon_{r}=30.43$ and a conductivity of $\sigma=1.503 \mathrm{~S} / \mathrm{m}$.

The FBSA results are obtained considering the horizontal and vertical cuts of the spherical pattern of an isotropic antenna $\left(P_{t}=50 \mathrm{~W}\right)$, located at the left-most end of the surfaces, for TM and TE polarization cases, respectively.

Both of the terrains are real life profiles so, for the downhill one the source is located at the coordinates $\left(40^{\circ}: 3^{\prime}: 18^{\prime \prime}\right)$ North and $\left(33^{\circ}: 2^{\prime}: 31.2^{\prime \prime}\right)$ East, around Ankara. The coordinates of the source for the uphill profile are $\left(37^{\circ}: 52^{\prime}: 33^{\prime \prime}\right)$ North and $\left(32^{\circ}: 25^{\prime}: 12^{\prime \prime}\right)$ East, around Konya.

In order to examine propagation models, the FBSA solution is taken as the reference. Simulation results of empirical propagation models have been obtained using the computer tool, Spectrum Engineering System, namely BILSPECT developed at the Communications and Spectrum Management Research Center of Bilkent University.

Firstly, in order to find out the best empirical propagation curve, the multiple diffraction correction method is set to Epstein-Peterson. Then, the field strength predictions are evaluated using Free Space Propagation model (FS), ITU-R recommendations; ITUR370, ITUR529, ITUR1546, and FCC Curves.

Figures 4.11-4.15 show these comparisons over the downhill profile, while Figures 4.16-4.20 illustrate examinations over the uphill one; for the TM and TE polarization in the $f=200 \mathrm{MHz}$ case. 
(a) non-PEC Terrain Profile

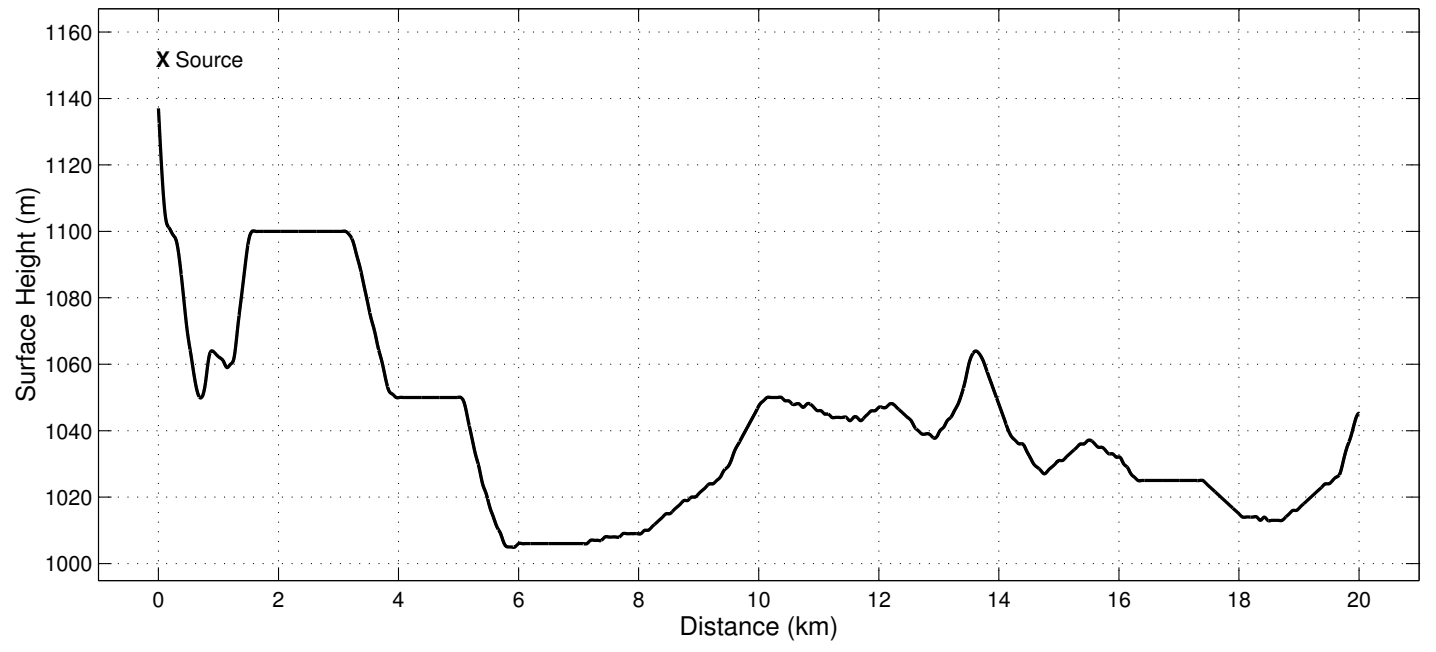

(b) TM Polarized Isotropic Radiator

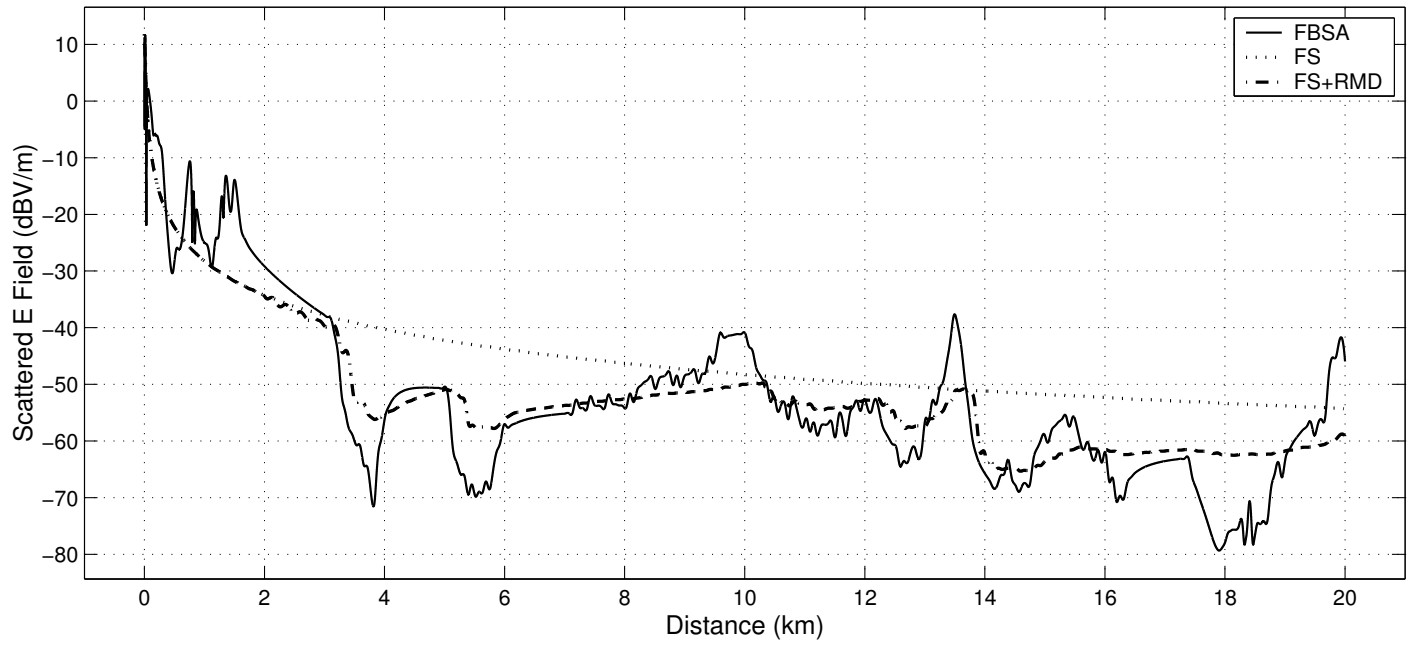

(c) TE Polarized Isotropic Radiator

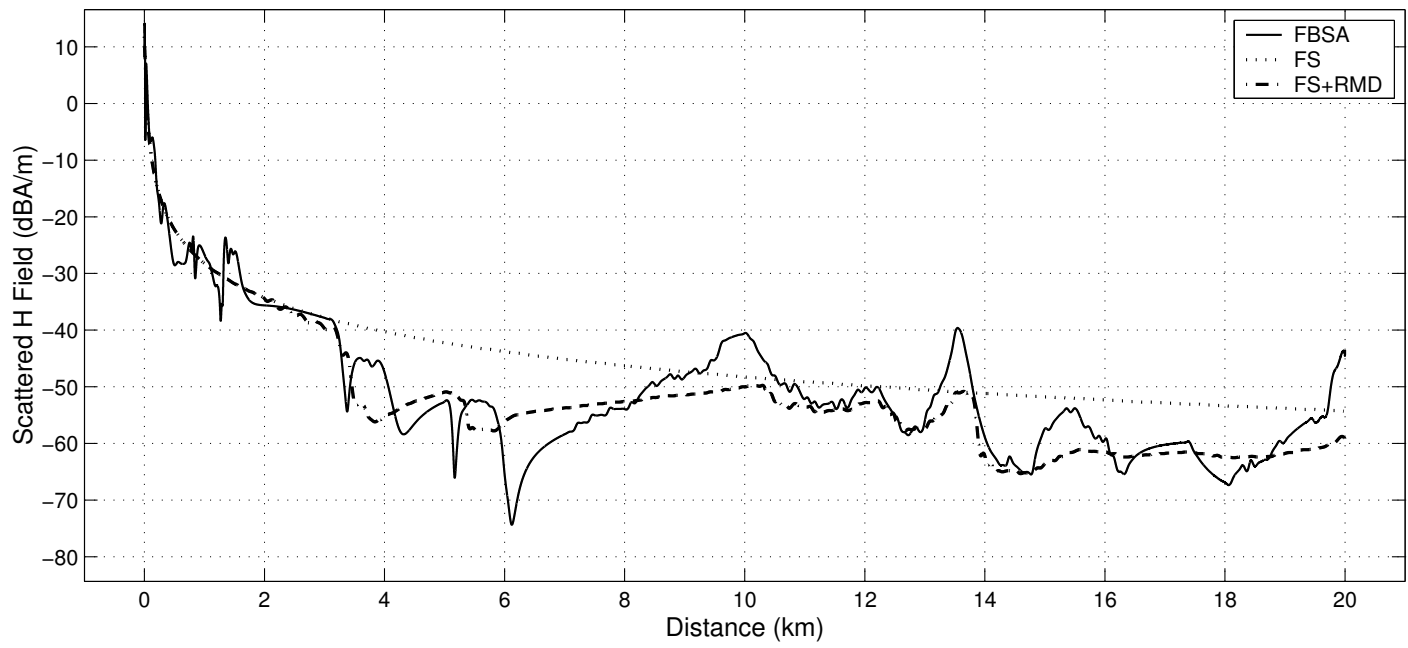

Figure 4.11: FreeSpace vs. IE over downhill terrain for $200 \mathrm{MHz}$. 
(a) non-PEC Terrain Profile

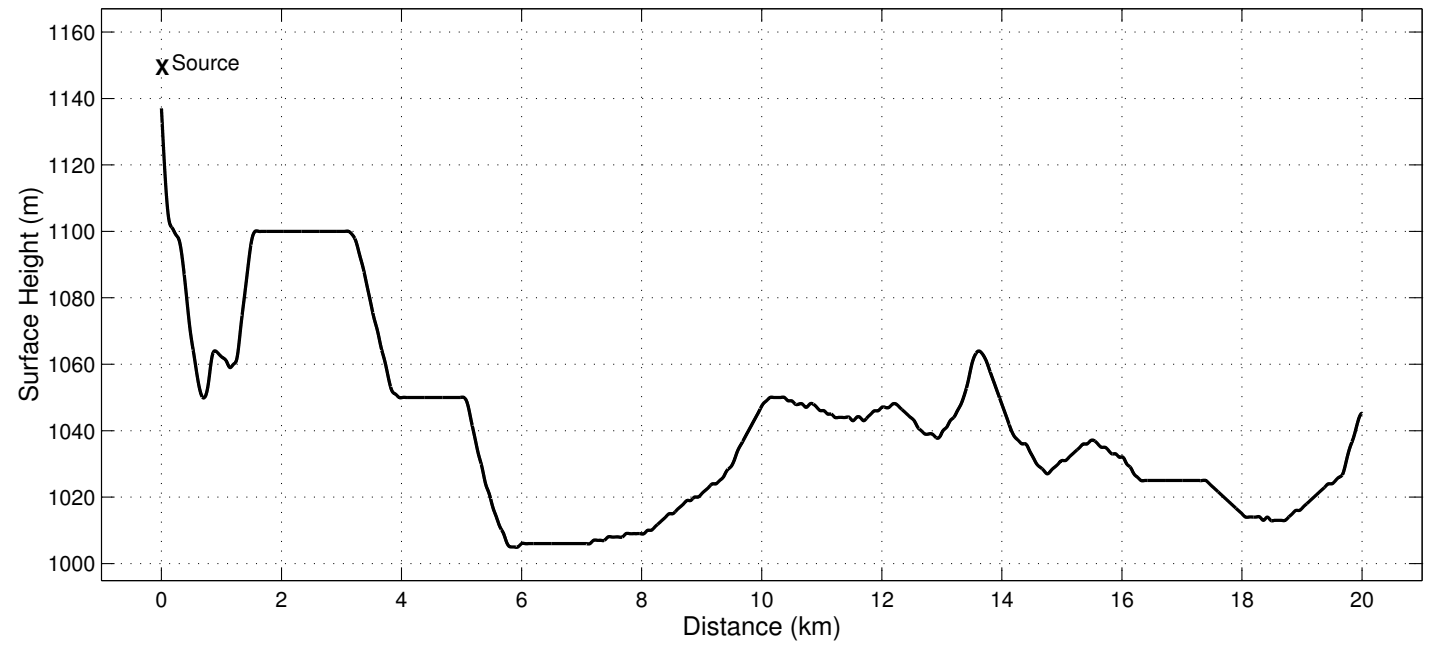

(b) TM Polarized Isotropic Radiator

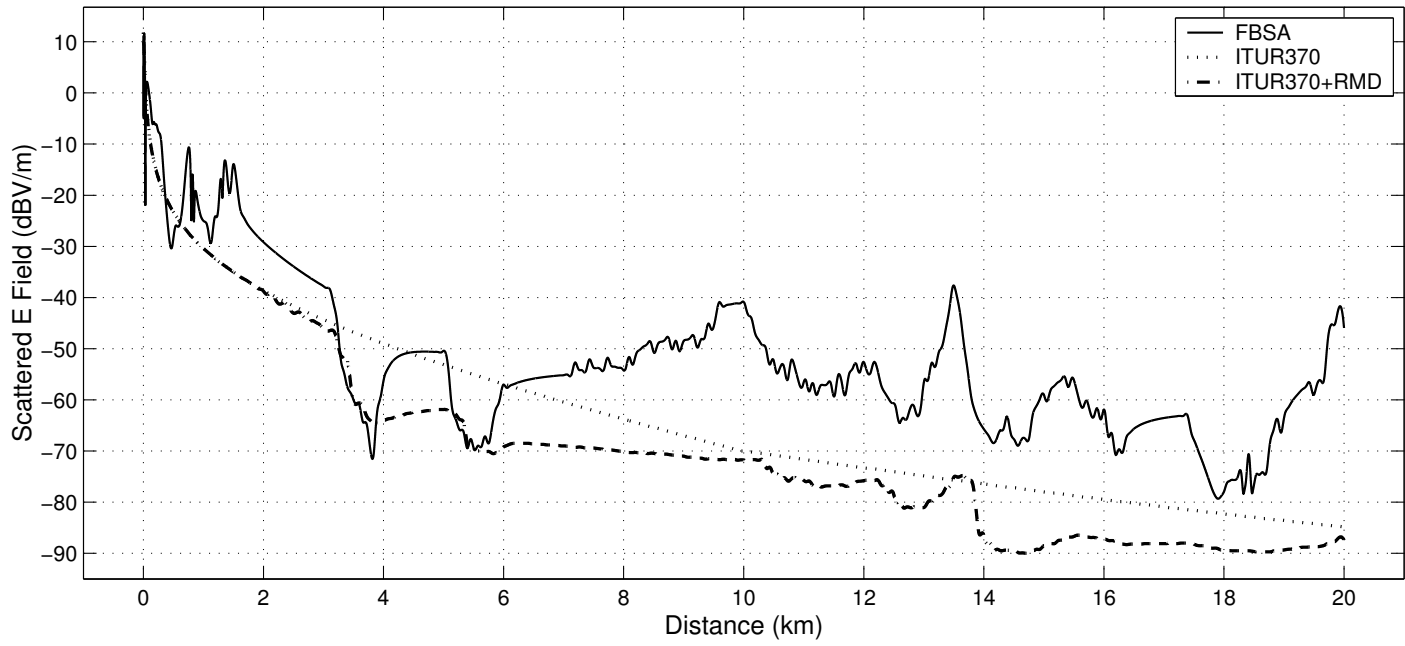

(c) TE Polarized Isotropic Radiator

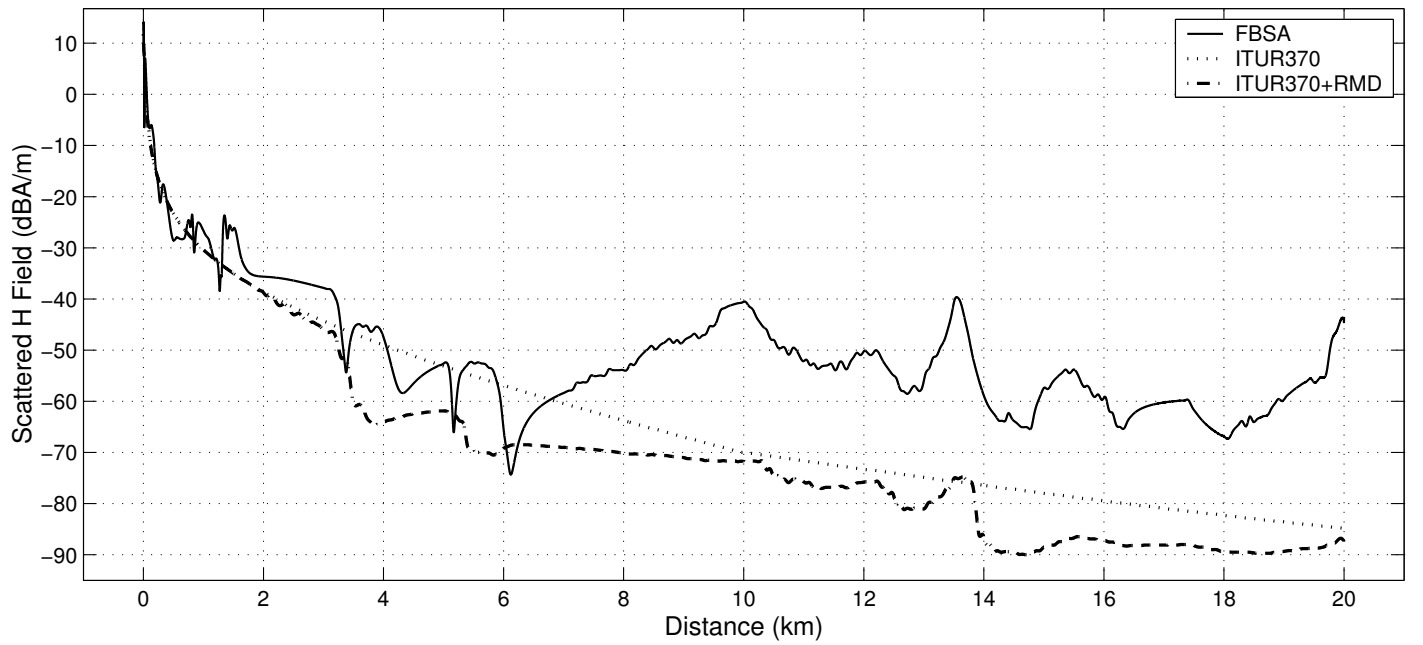

Figure 4.12: ITUR370 vs. IE over downhill terrain for $200 \mathrm{MHz}$. 
(a) non-PEC Terrain Profile

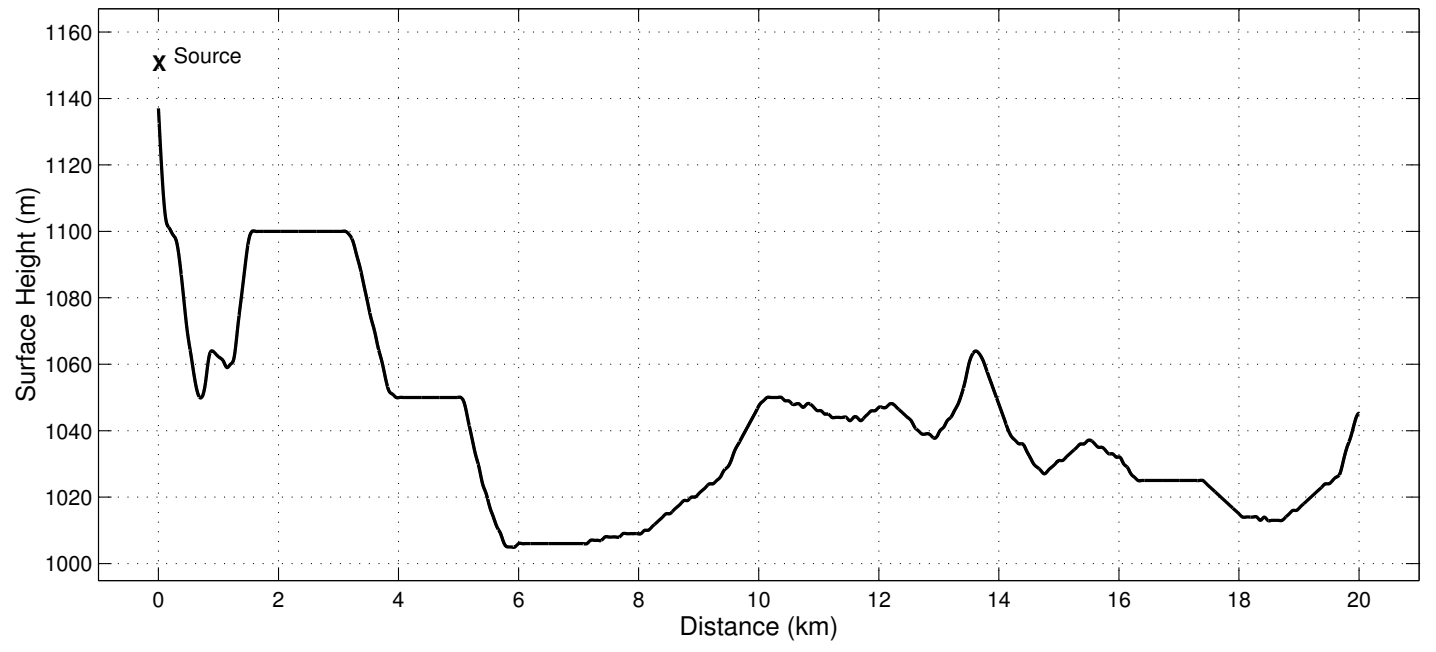

(b) TM Polarized Isotropic Radiator

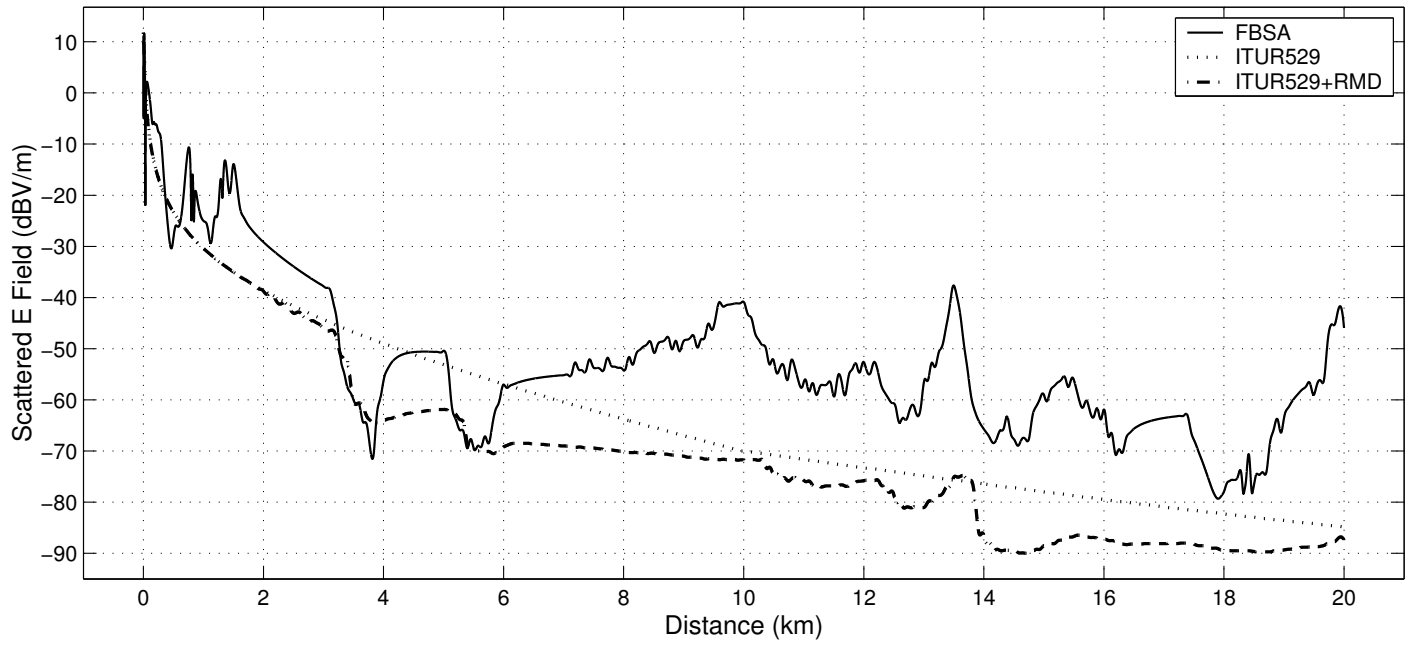

(c) TE Polarized Isotropic Radiator

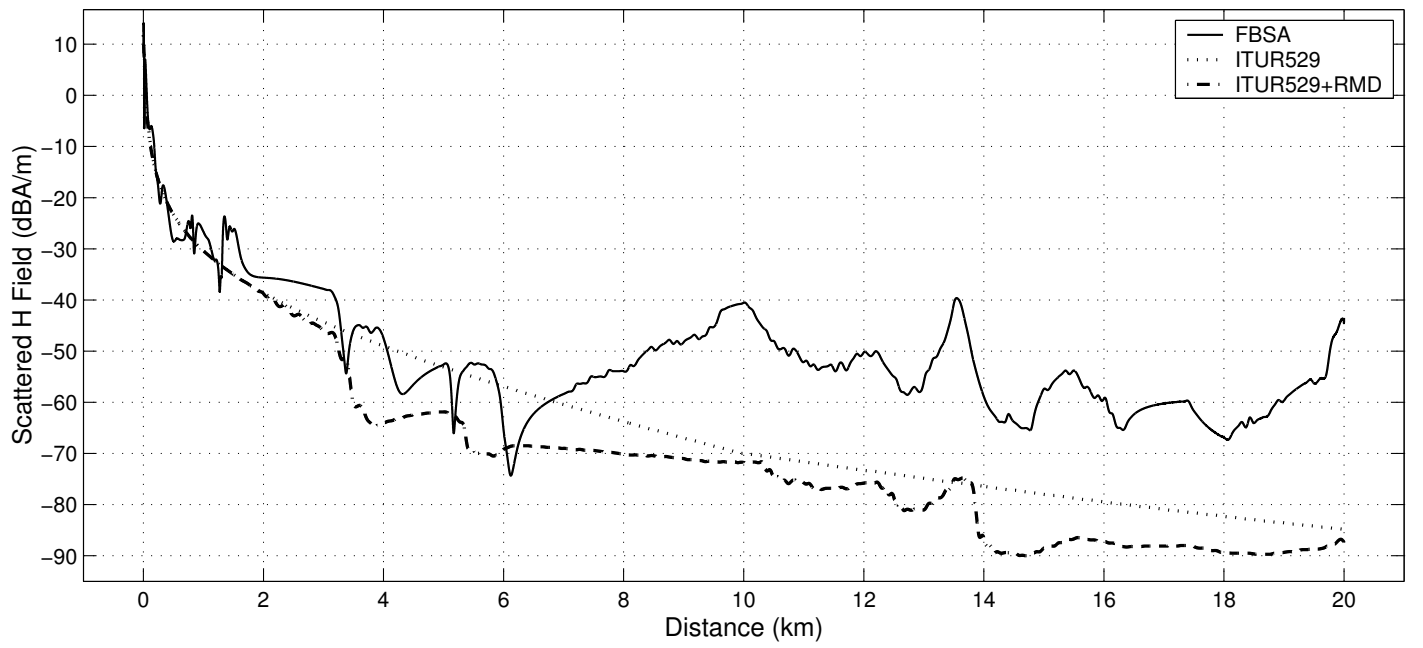

Figure 4.13: ITUR529 vs. IE over downhill terrain for $200 \mathrm{MHz}$. 
(a) non-PEC Terrain Profile

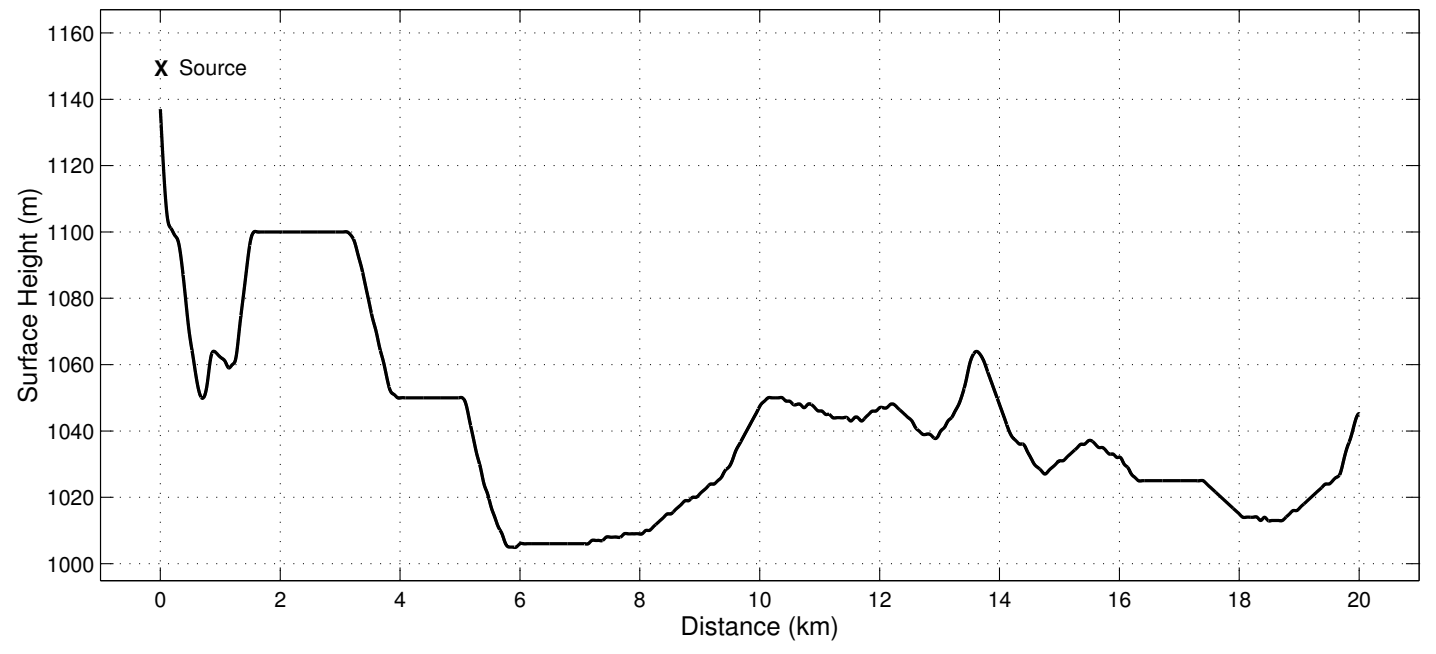

(b) TM Polarized Isotropic Radiator

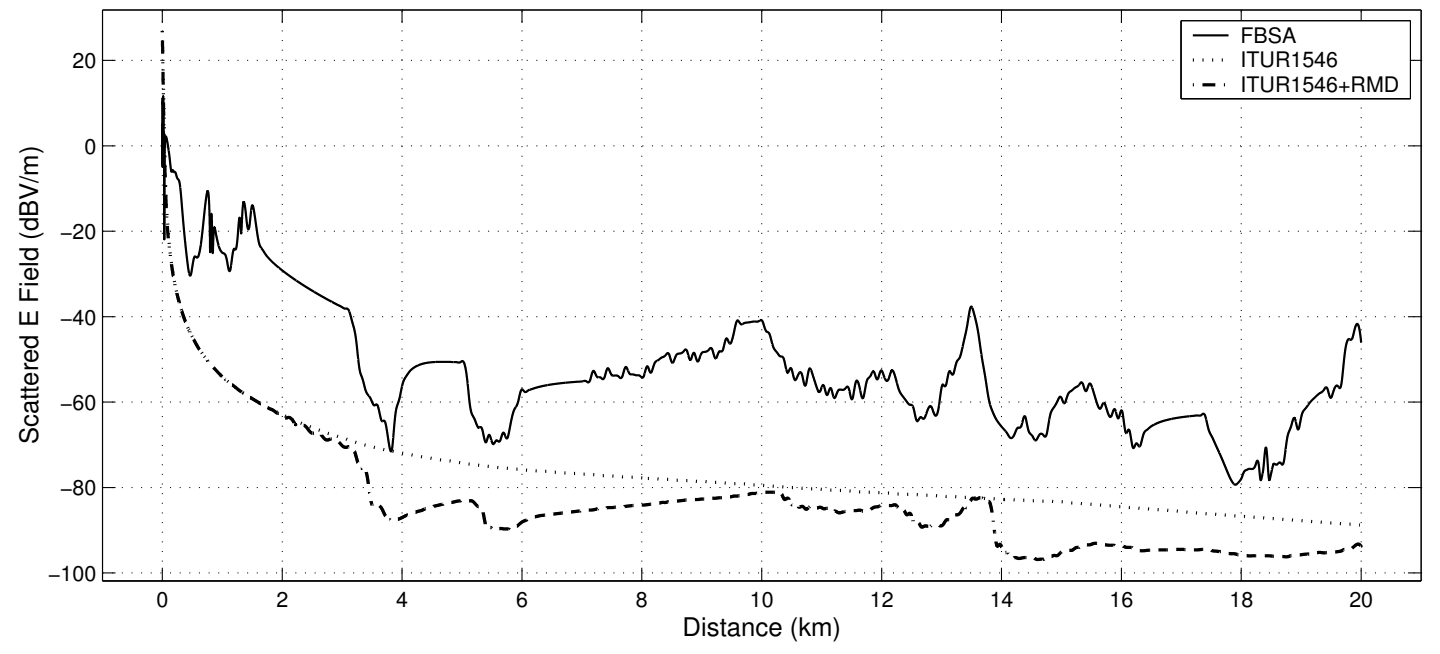

(c) TE Polarized Isotropic Radiator

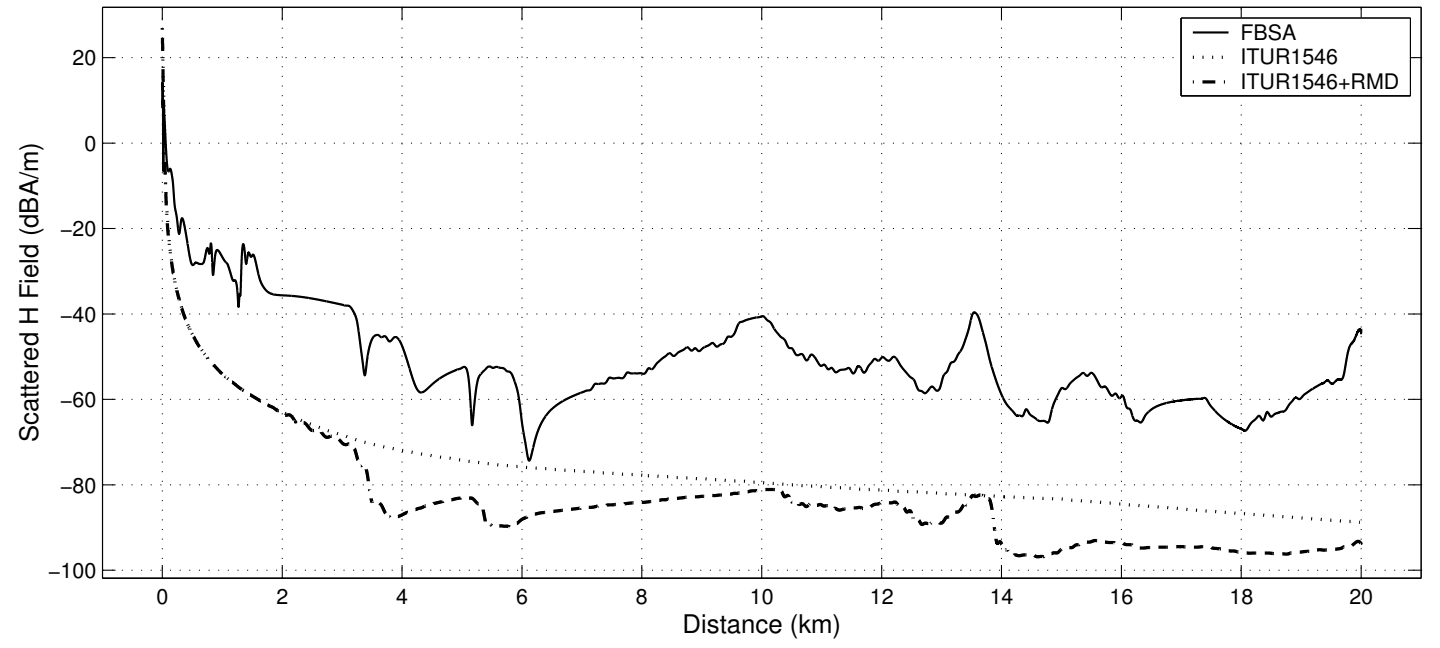

Figure 4.14: ITUR1546 vs. IE over downhill terrain for $200 \mathrm{MHz}$. 
(a) non-PEC Terrain Profile

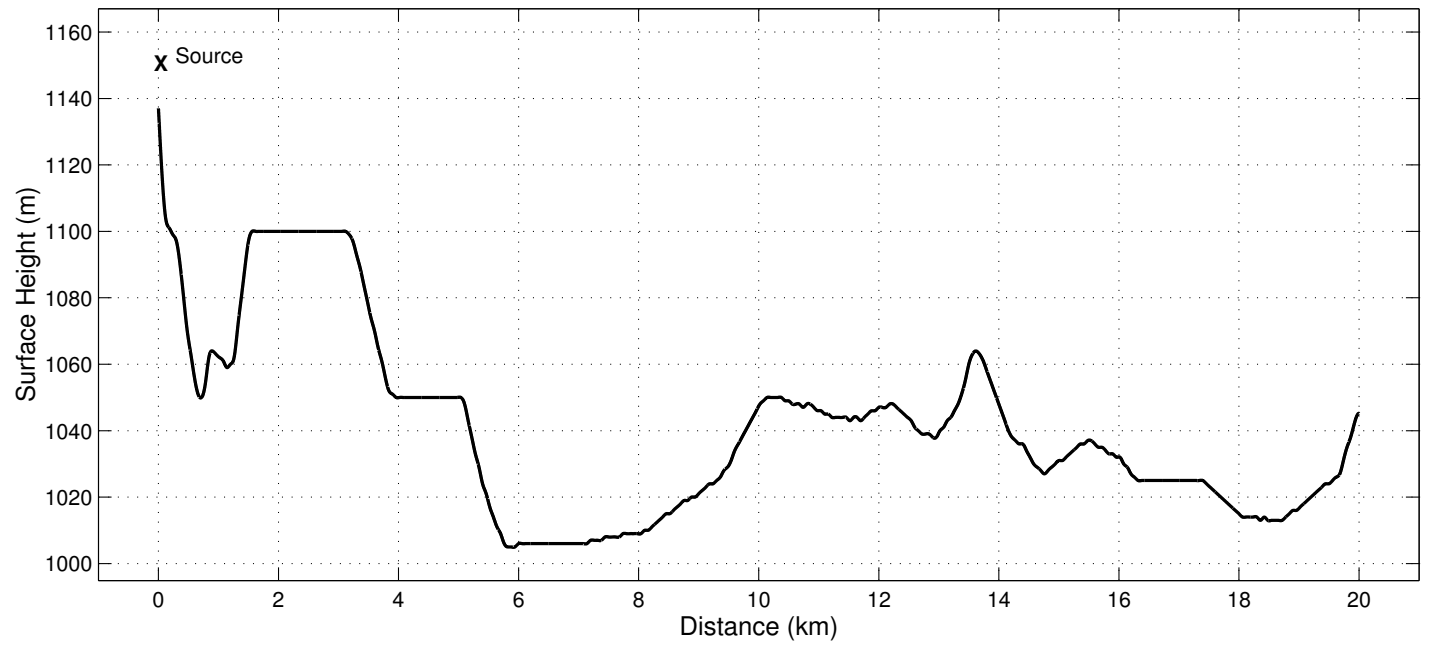

(b) TM Polarized Isotropic Radiator

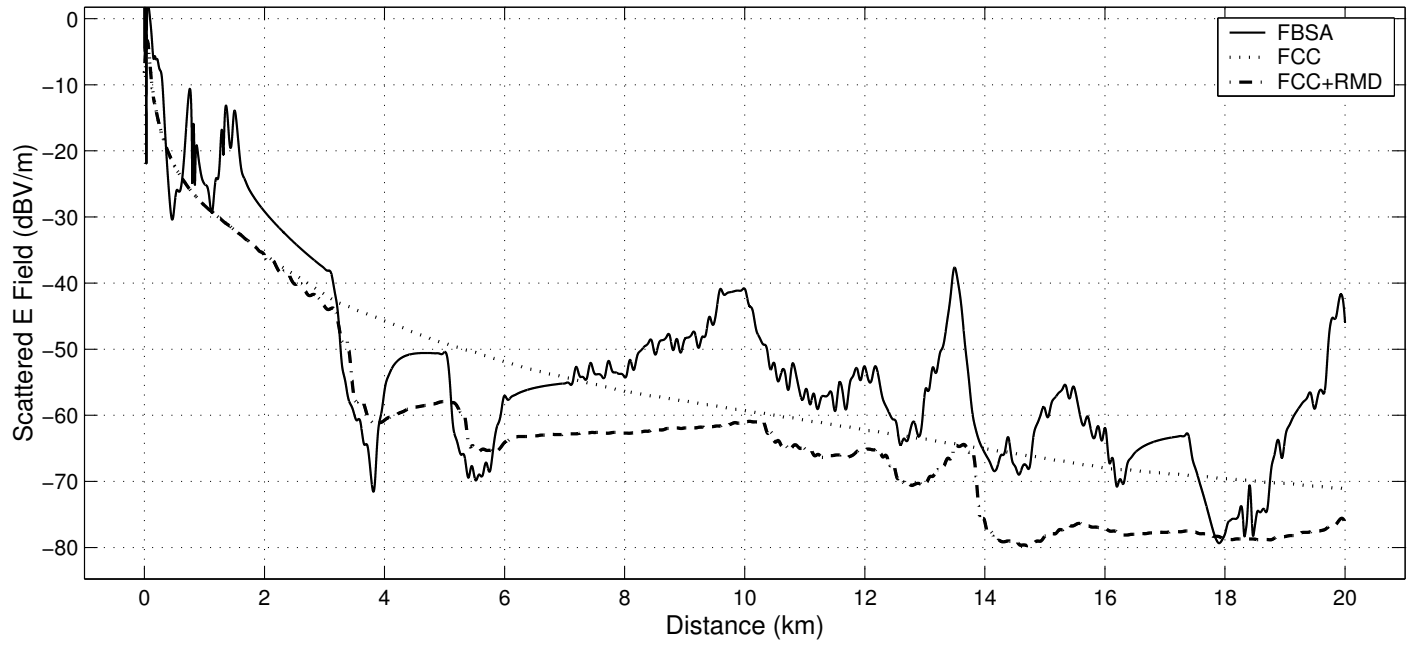

(c) TE Polarized Isotropic Radiator

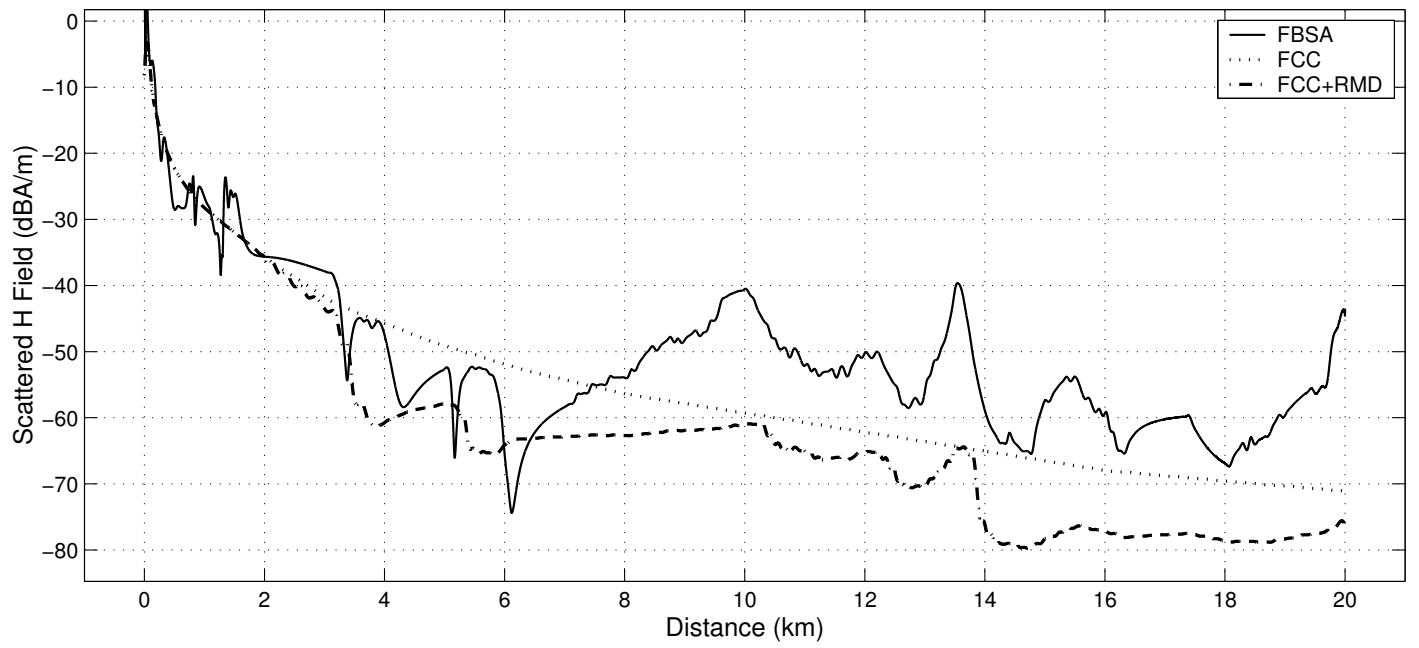

Figure 4.15: FCC vs. IE over downhill terrain for $200 \mathrm{MHz}$. 
(a) non-PEC Terrain Profile

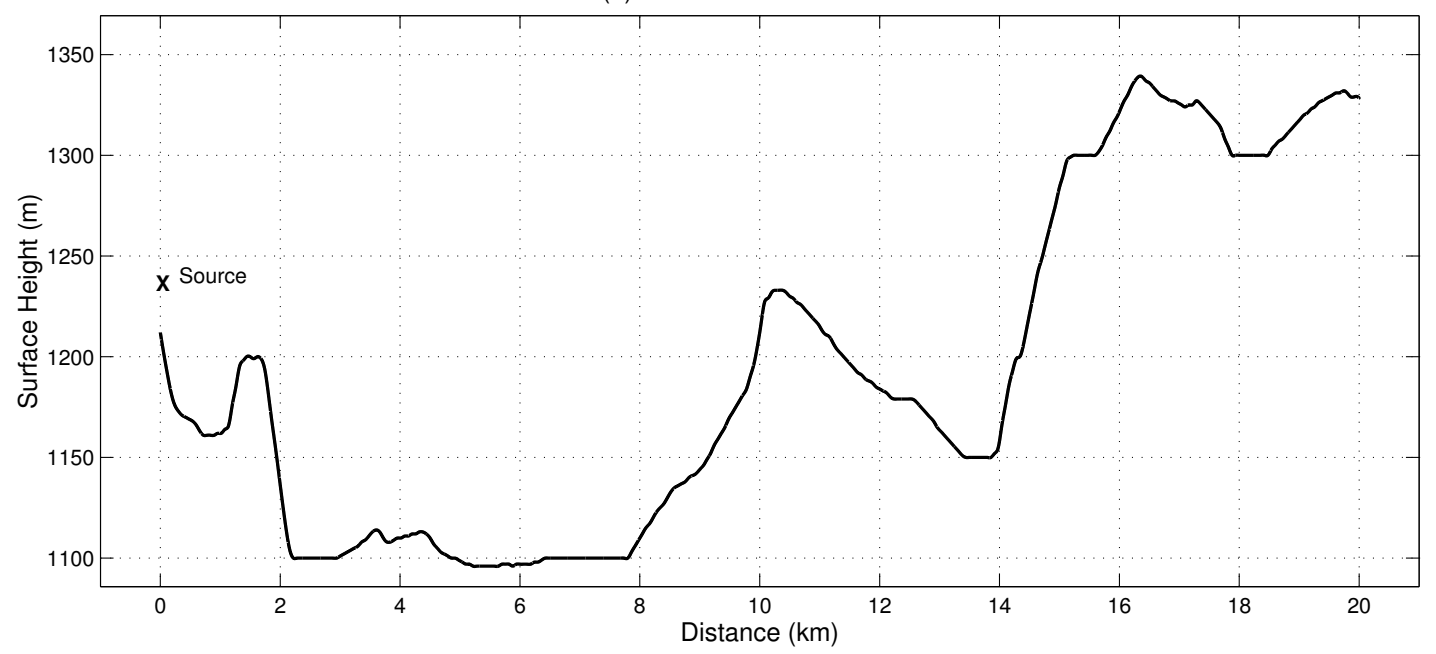

(b) TM Polarized Isotropic Radiator

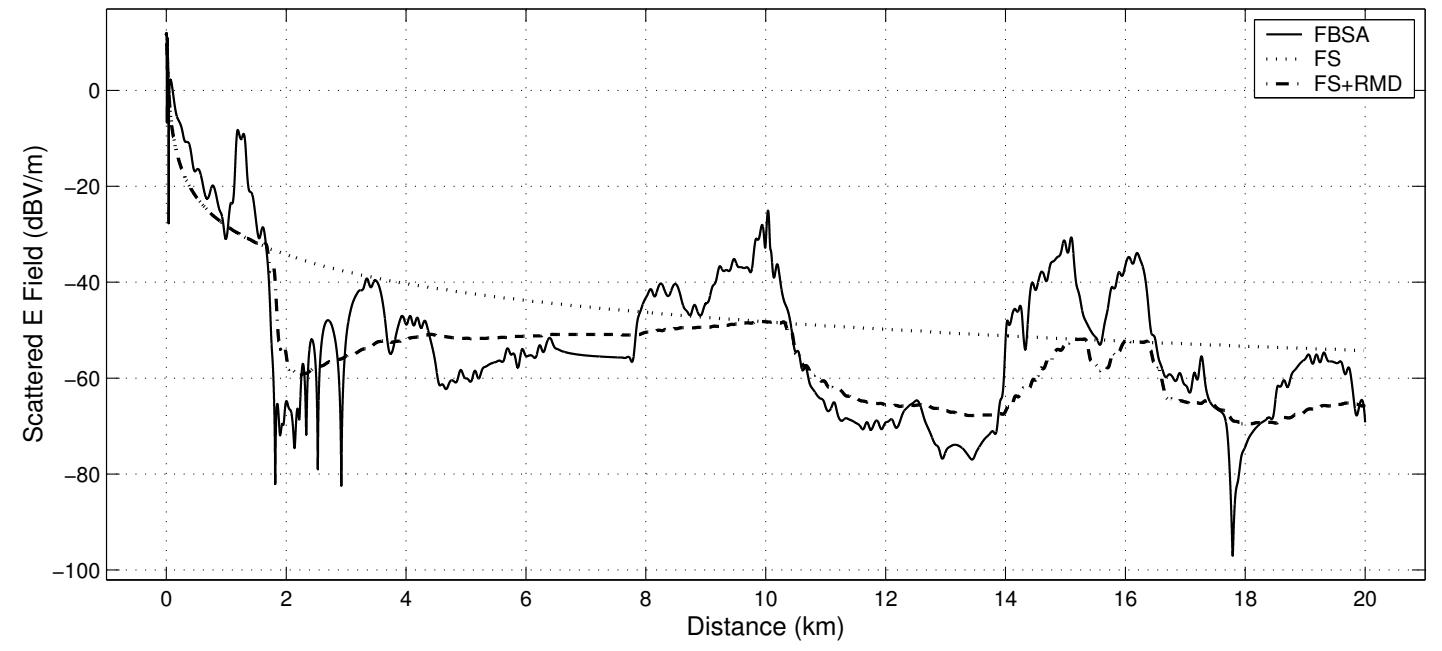

(c) TE Polarized Isotropic Radiator

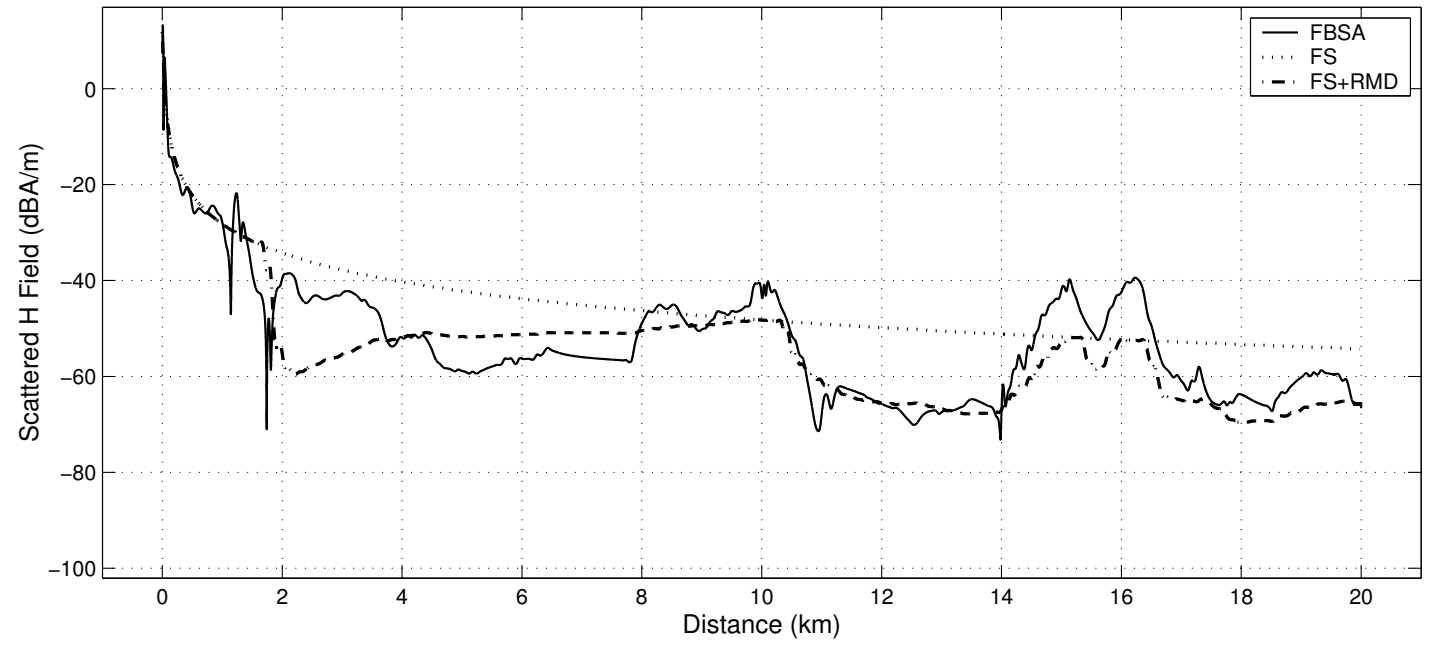

Figure 4.16: FreeSpace vs. IE over uphill terrain for $200 \mathrm{MHz}$. 
(a) non-PEC Terrain Profile

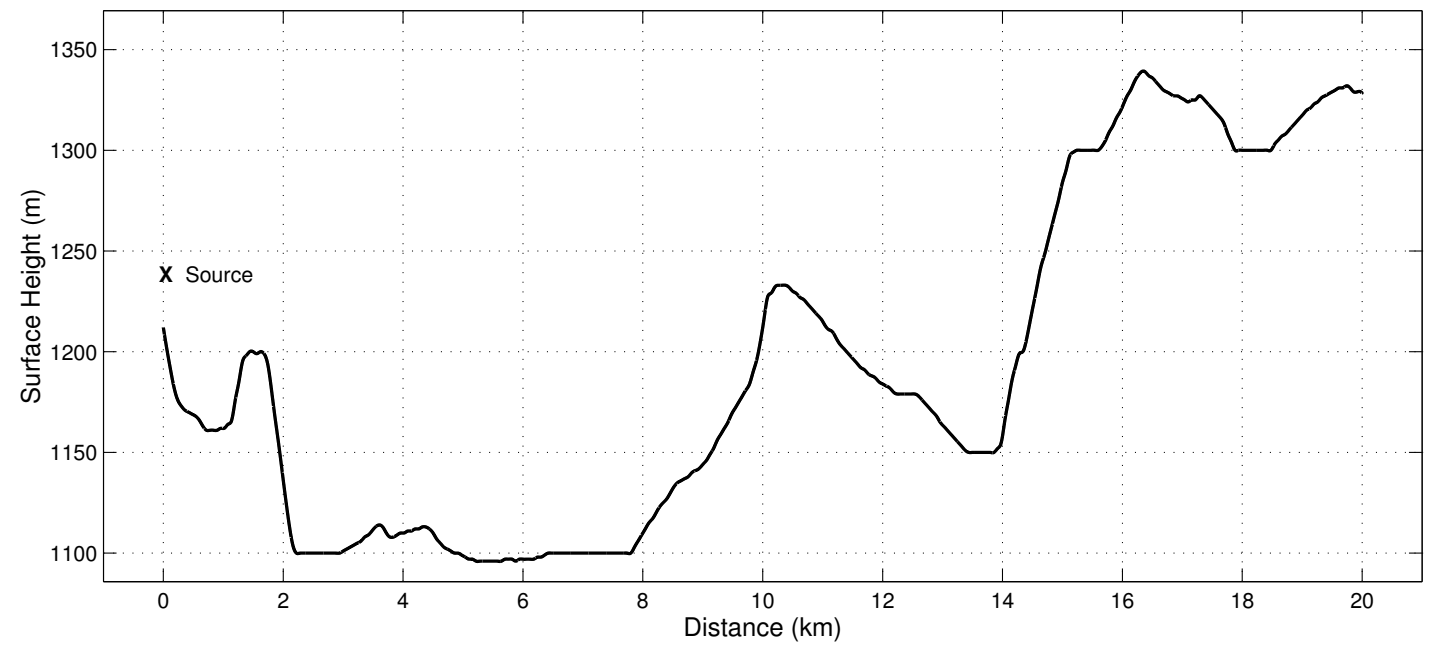

(b) TM Polarized Isotropic Radiator

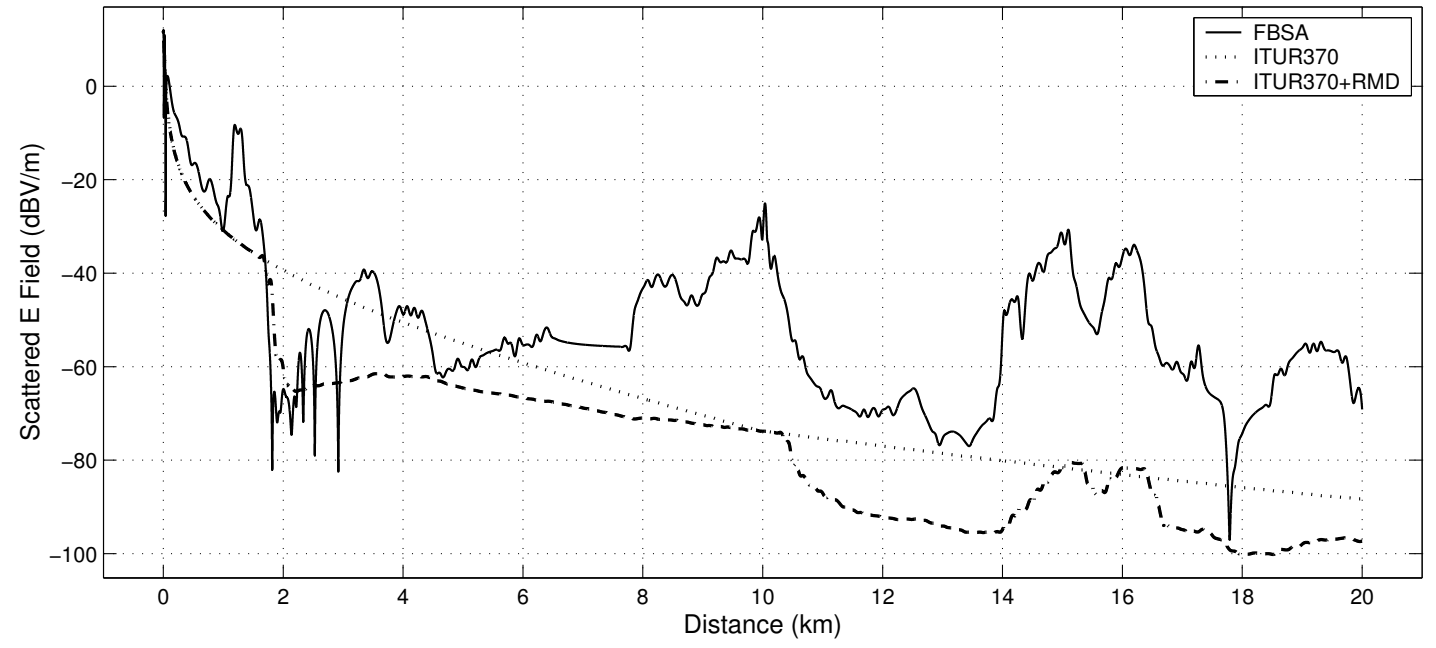

(c) TE Polarized Isotropic Radiator

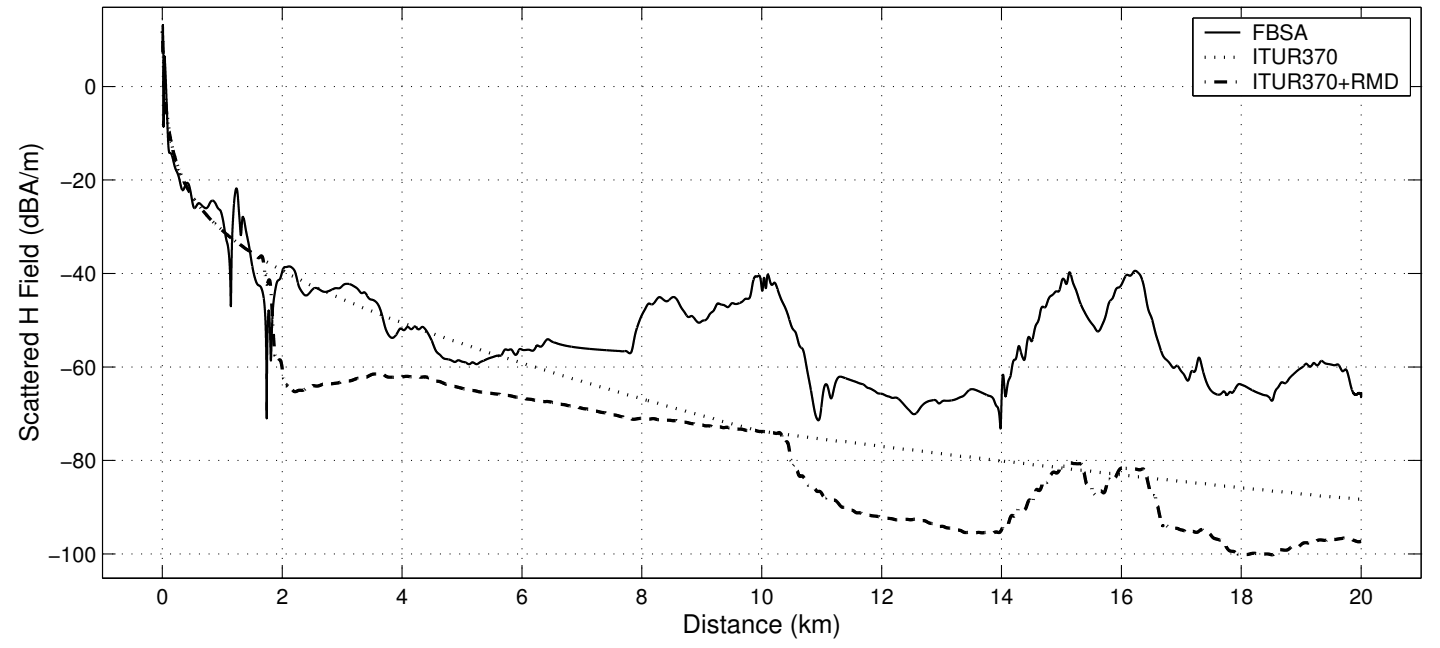

Figure 4.17: ITUR370 vs. IE over uphill terrain for $200 \mathrm{MHz}$. 
(a) non-PEC Terrain Profile

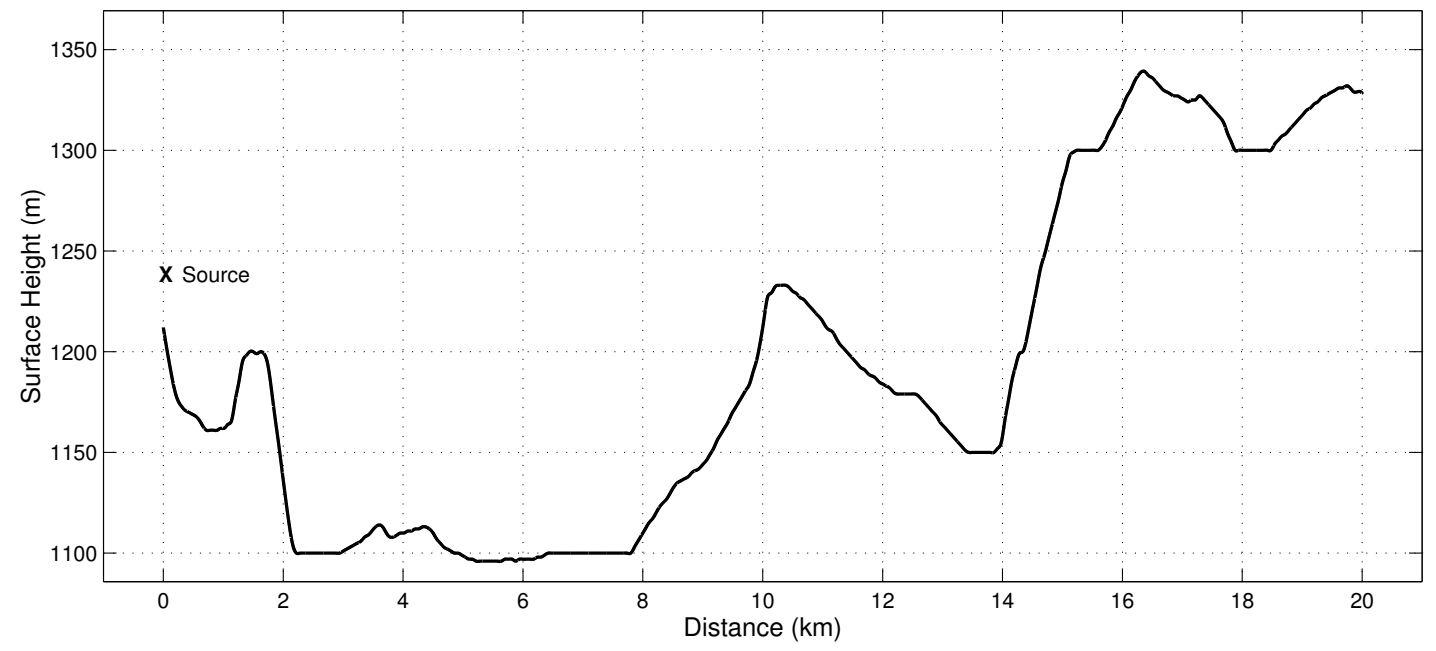

(b) TM Polarized Isotropic Radiator

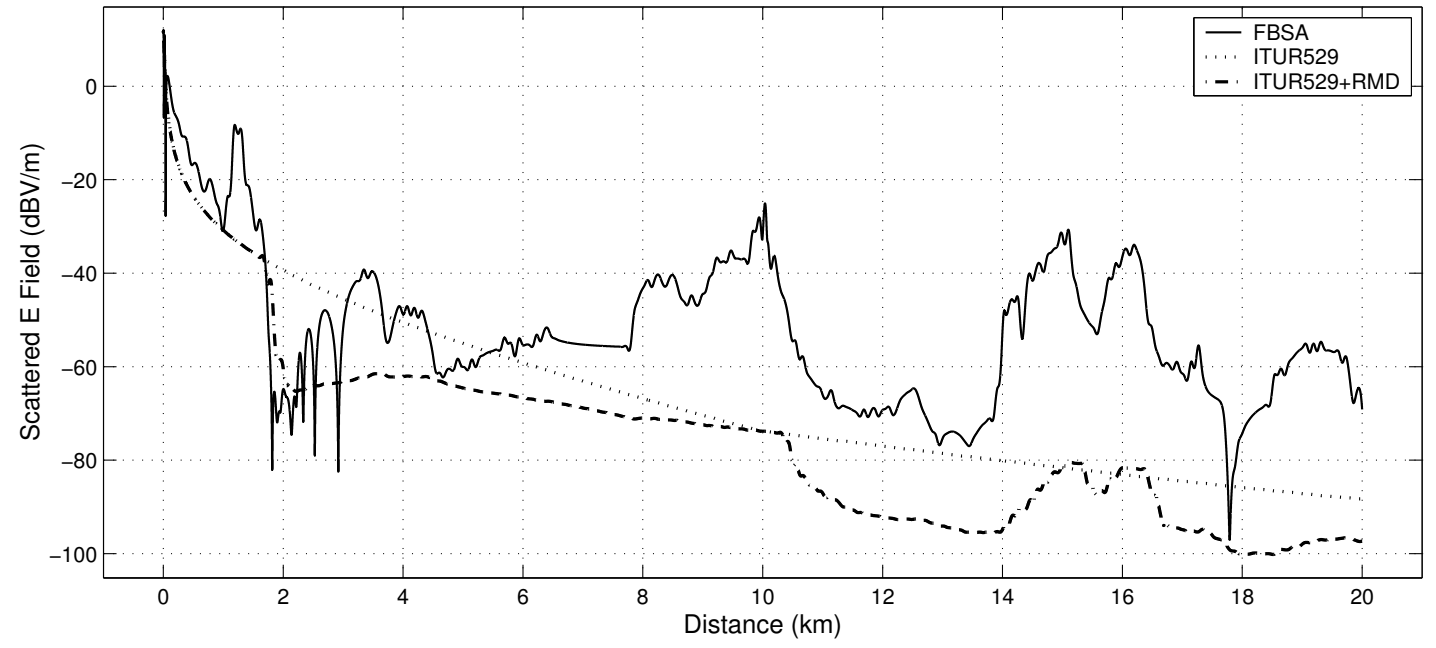

(c) TE Polarized Isotropic Radiator

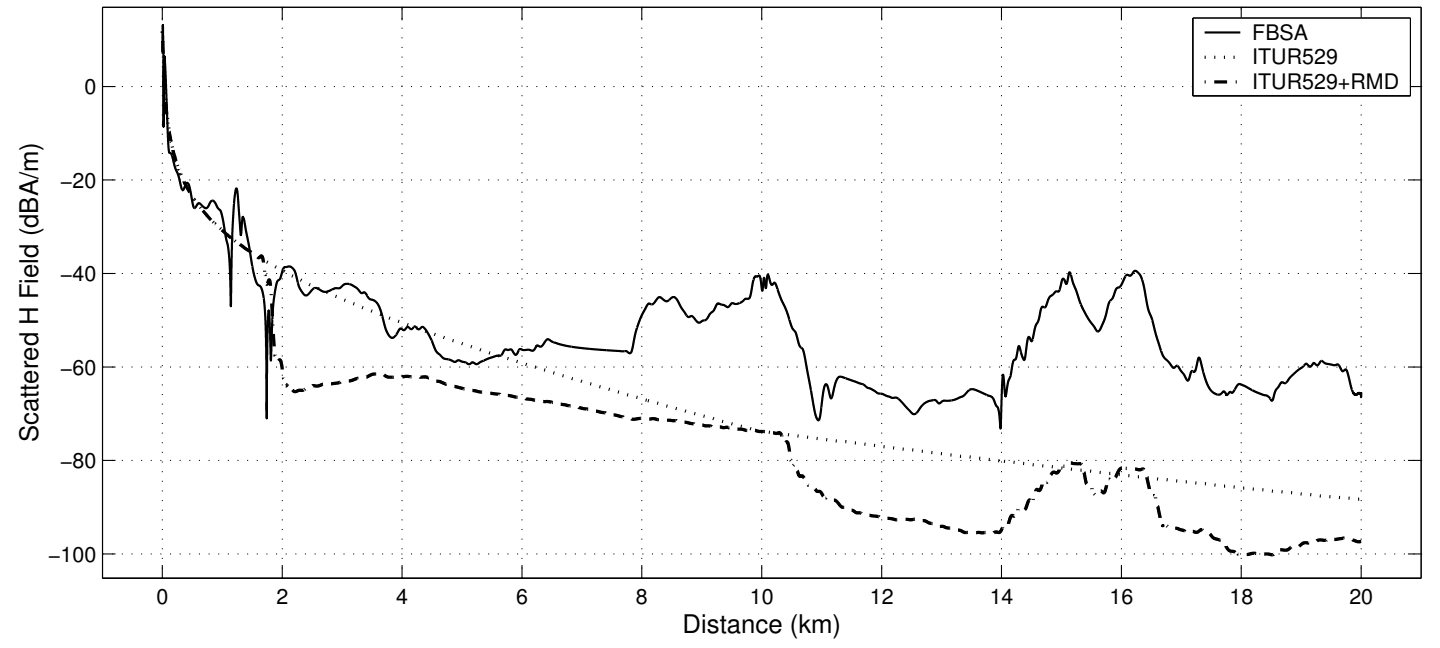

Figure 4.18: ITUR529 vs. IE over uphill terrain for $200 \mathrm{MHz}$. 
(a) non-PEC Terrain Profile

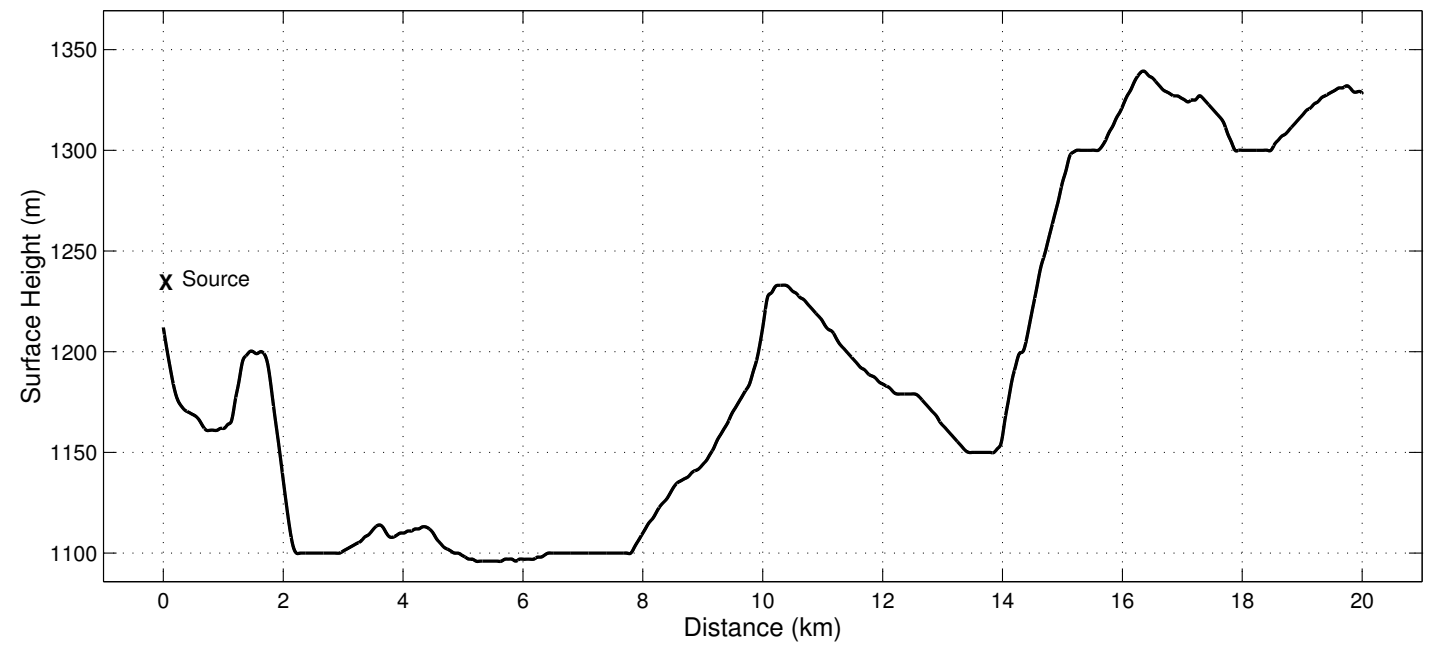

(b) TM Polarized Isotropic Radiator

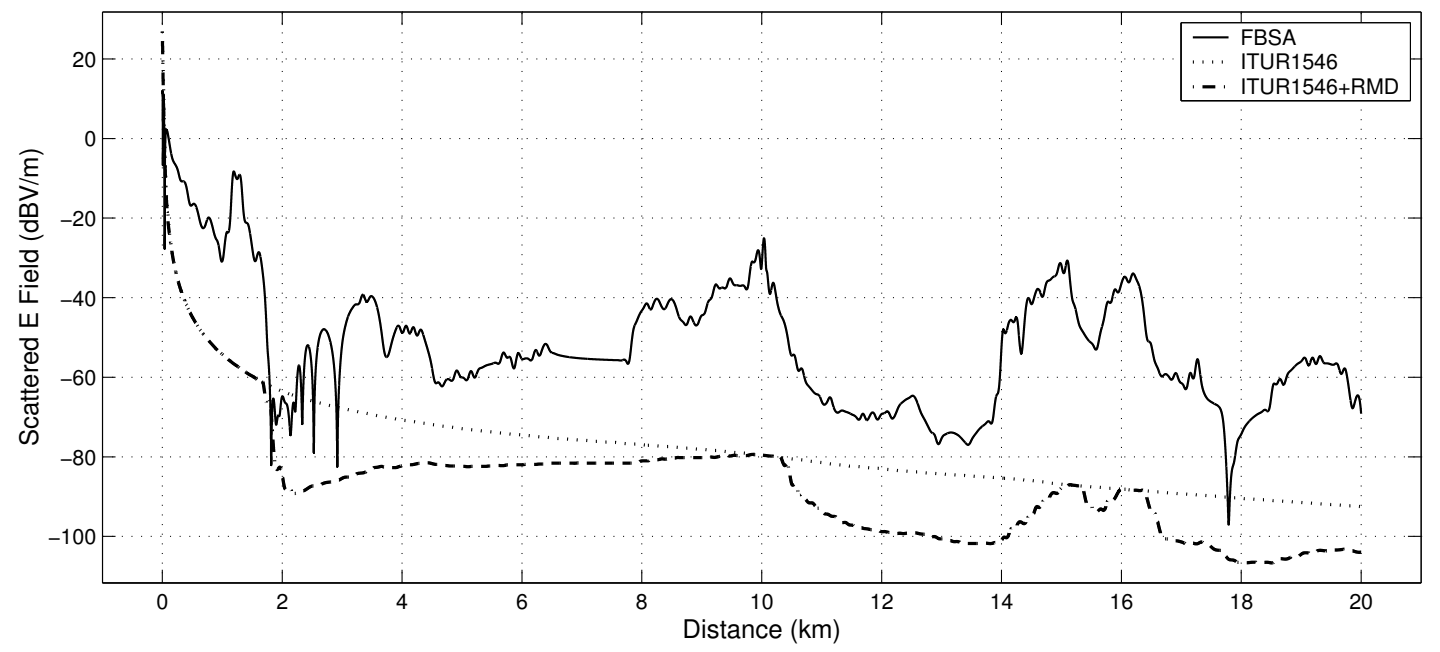

(c) TE Polarized Isotropic Radiator

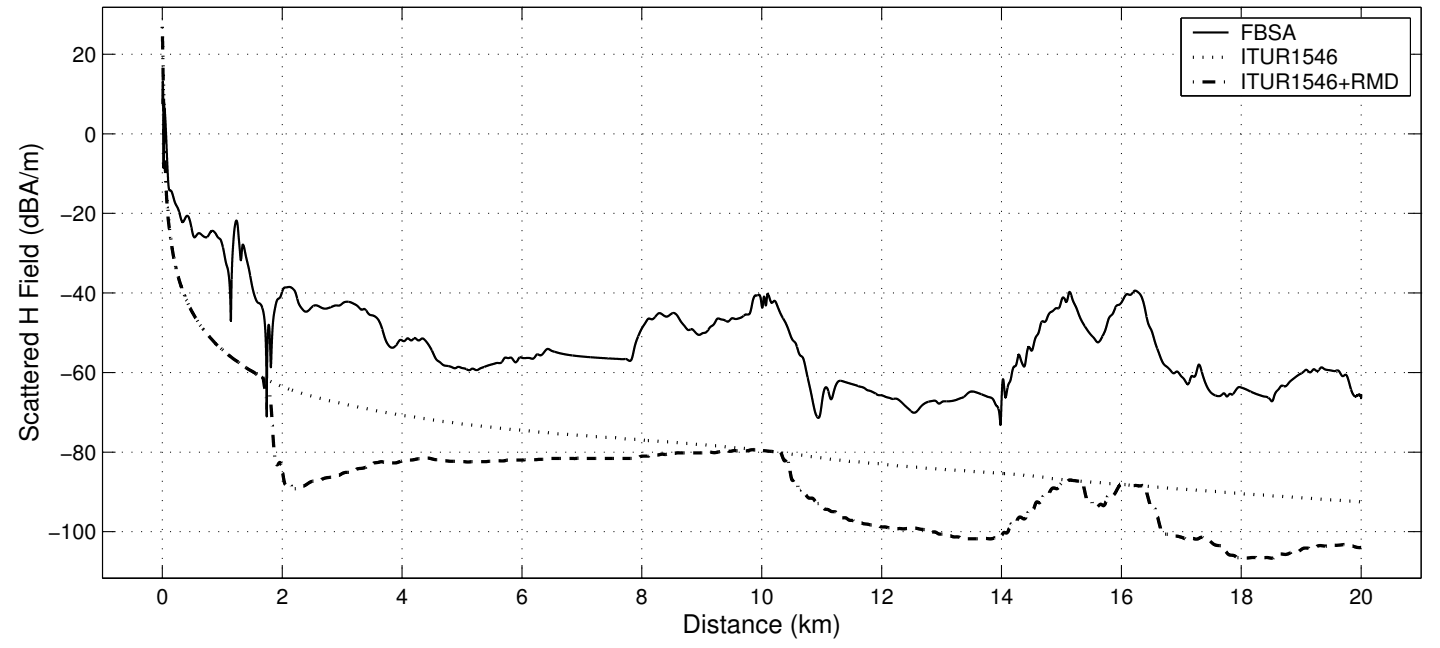

Figure 4.19: ITUR1546 vs. IE over uphill terrain for $200 \mathrm{MHz}$. 
(a) non-PEC Terrain Profile

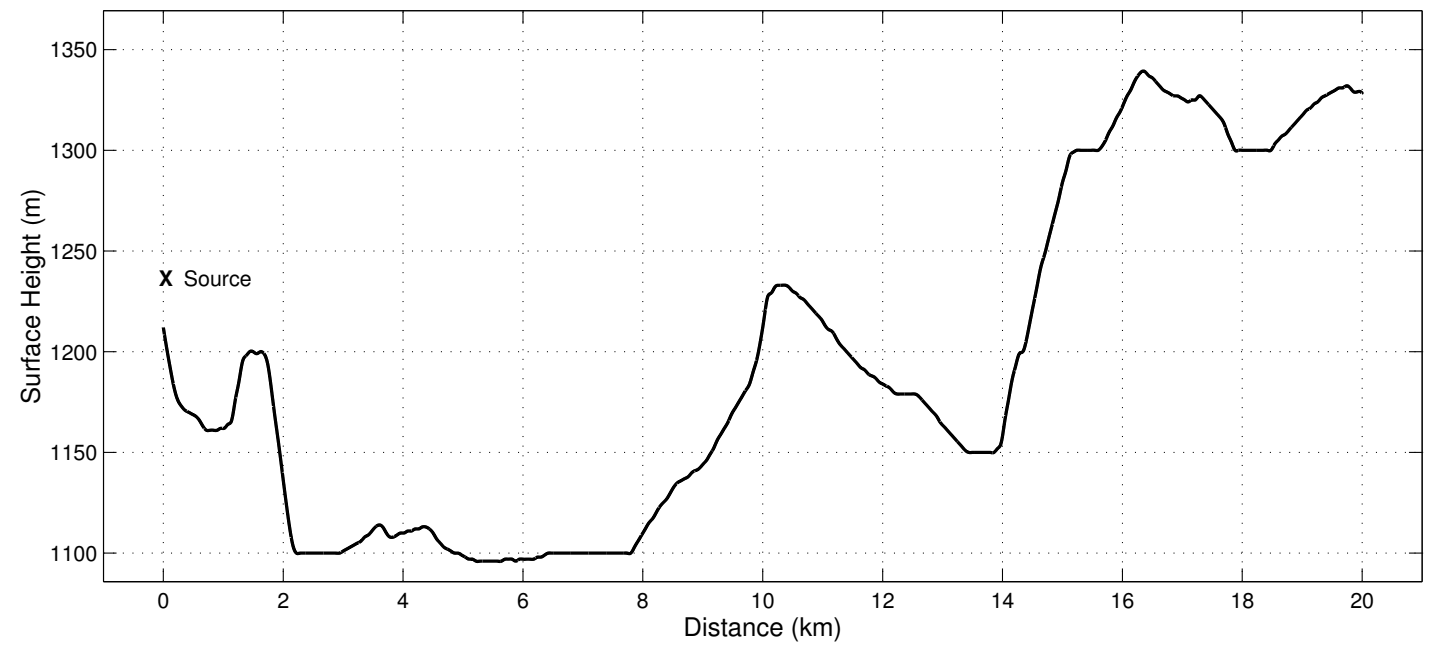

(b) TM Polarized Isotropic Radiator

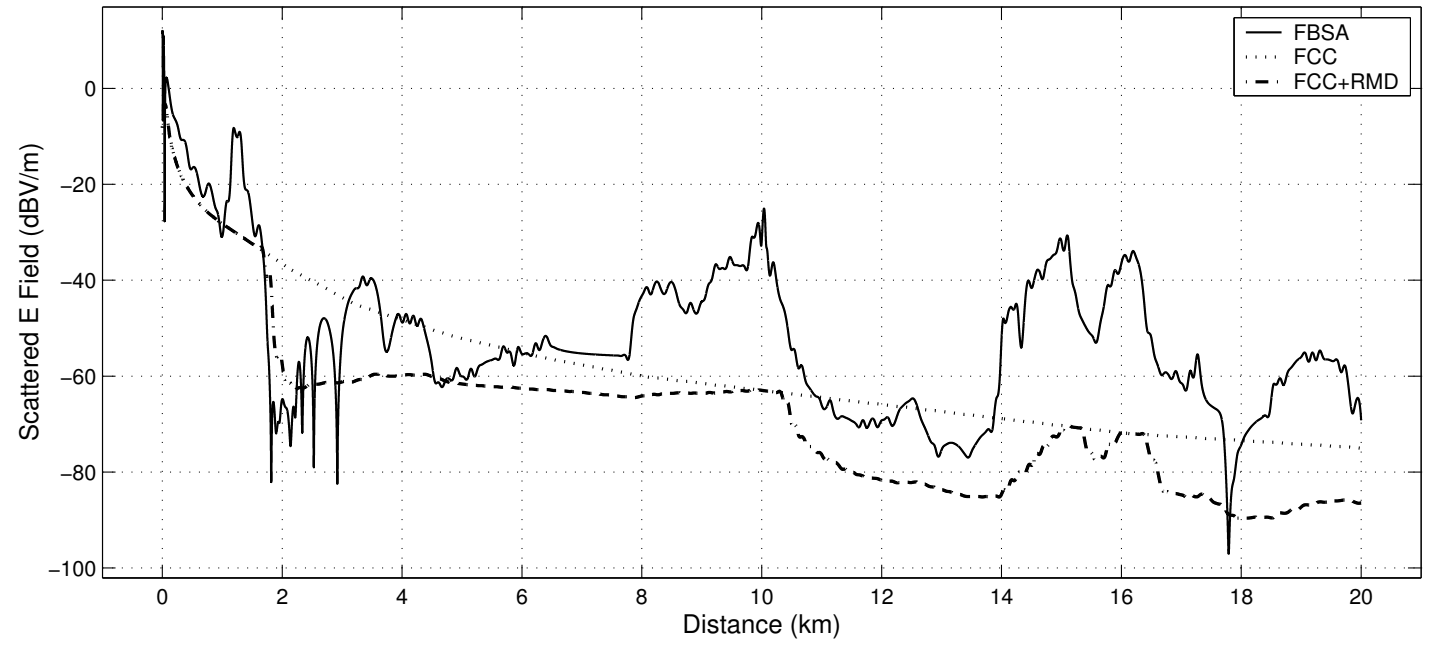

(c) TE Polarized Isotropic Radiator

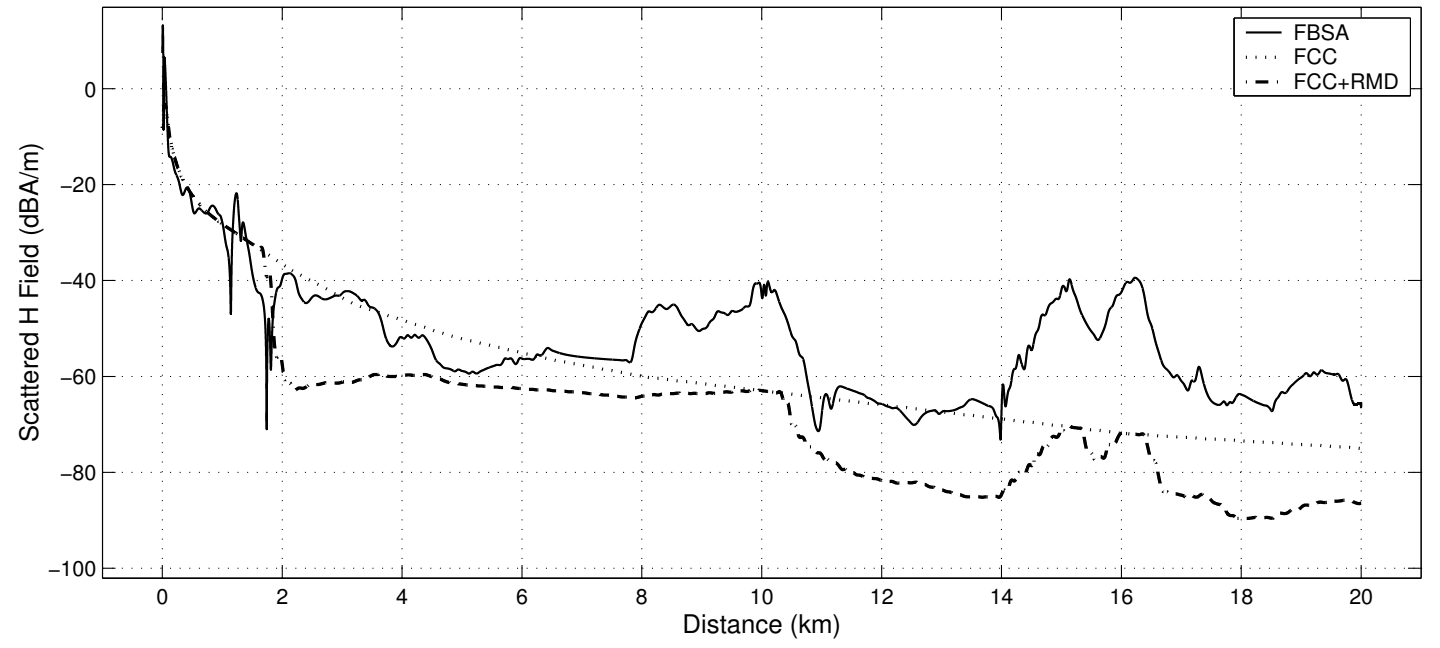

Figure 4.20: FCC vs. IE over uphill terrain for $200 \mathrm{MHz}$. 
The absolute error is used as the accuracy criterion to compare the empirical propagation models with the FBSA . Table 4.2 shows the absolute error values for different propagation models and polarizations over uphill (UH) and downhill (DH) profiles for $200 \mathrm{MHz}$.

\begin{tabular}{|c|c|c|c|c|}
\hline Absolute Error (\%) & DH (TM) & DH (TE) & UH (TM) & UH (TE) \\
\hline FreeSpace & 21.46 & 16.56 & 26.41 & 19.49 \\
\hline ITUR-370 & 28.82 & 32.85 & 41.13 & 35.70 \\
\hline ITUR-529 & 28.82 & 32.85 & 41.13 & 35.70 \\
\hline ITUR-1546 & 46.33 & 50.56 & 52.88 & 48.98 \\
\hline FCC & 17.11 & 17.83 & 29.83 & 21.82 \\
\hline FreeSpace + EpsPet & 11.61 & 9.22 & 16.69 & 12.03 \\
\hline ITUR-370 + EpsPet & 36.13 & 41.79 & 49.92 & 47.73 \\
\hline ITUR-529 + EpsPet & 36.13 & 41.79 & 49.92 & 47.73 \\
\hline ITUR-1546 + EpsPet & 56.91 & 62.62 & 66.43 & 65.04 \\
\hline FCC + EpsPet & 21.89 & 26.27 & 36.06 & 33.35 \\
\hline
\end{tabular}

Table 4.2: Absolute error values for $200 \mathrm{MHz}$

The total field attenuation for propagation models is the sum of the field attenuation of the empirical model, the reflection loss and the diffraction loss.

Therefore, if the field strength values predicted by an empirical model are at a lower level in magnitude than the reference solution, using RMD methods increases the error generated.

Thus, empirical model having the highest field strength level should be chosen. Multiple diffraction methods yield satisfactory losses, even if selecting an empirical model having a very high level of field strength.

It is observed that for an isotropic source having $200 \mathrm{MHz}$ operating frequency, using FCC curves or free space propagation model without RMD corrections is preferable than the others over both uphill and downhill profiles for TE and TM polarization cases.

However, free space propagation loss with multiple diffraction is the most preferable model among others. Note that, modified Hata methods ITUR-370 and 529 models give the same results for $f=200 \mathrm{MHz}$ and the worst model seems 
to be the ITUR-1546. Also, it is worth mentioning that, the empirical propagation models and multiple diffraction corrections do not respond to changes in polarization, so they obtain the same field attenuation results for both TM and TE polarizations.

Secondly, $f=500 \mathrm{MHz}$ case is examined. Figure 4.21 (b) and 4.22 (b) illustrate the deformed integration paths of downhill and uphill profiles, respectively, for $500 \mathrm{MHz}$. Required parameters for the spectral acceleration are included in Table 4.3 for this case.

Comparing Tables 4.1 and 4.3, it is obvious that only terrain dependent values like saddle points are not changed but frequency dependent parameters like the inclination angle of the deformed path and number of integration samples are changed.

\begin{tabular}{|c|c|c|}
\hline & Downhill & Uphill \\
\hline Frequency $(\mathrm{MHz})$ & 500 & 500 \\
\hline$\lambda(\mathrm{m})$ & 0.6 & 0.6 \\
\hline width $(\lambda)$ & 33333.3 & 33333.3 \\
\hline$N$ & 333333 & 333333 \\
\hline$\phi_{\text {med }}(\mathrm{rad})$ & -0.003 & 0.011 \\
\hline$\phi_{s, \text { min }}(\mathrm{rad})$ & -0.336 & -0.233 \\
\hline$\phi_{s, \text { max }}(\mathrm{rad})$ & 0.169 & 0.209 \\
\hline$L_{s}(\lambda)$ & 8.333 & 8.333 \\
\hline Num. of integr. points & 286 & 366 \\
\hline$\delta(\mathrm{rad})$ & 0.159 & 0.104 \\
\hline
\end{tabular}

Table 4.3: Study Parameters for $500 \mathrm{MHz}$

Profiles are considered to be impedance surfaces of $\eta_{s}=25+j 20 \Omega$. At 500 $\mathrm{MHz}$, this impedance corresponds to a relative permittivity of $\epsilon_{r}=30.43$ and a conductivity of $\sigma=3.758 \mathrm{~S} / \mathrm{m}$.

The FBSA results are obtained considering the horizontal and vertical cuts of the spherical pattern of an isotropic antenna located at the left-most end of the surfaces with a transmitted power of $P_{t}=50$ Watts, for TM and TE polarization cases, respectively, using the parameters in Table 4.3. 

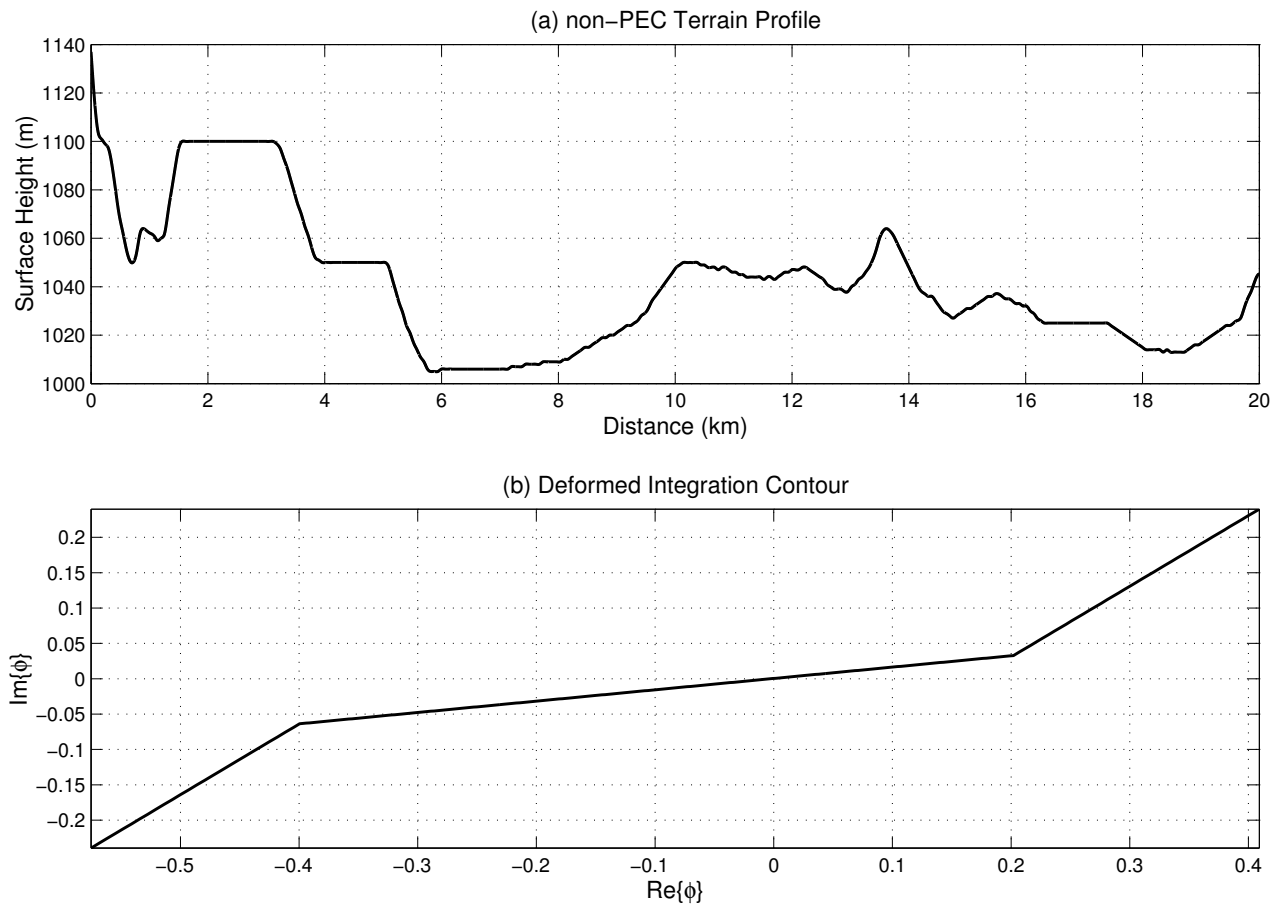

Figure 4.21: Deformed path of downhill profile at $500 \mathrm{MHz}$.
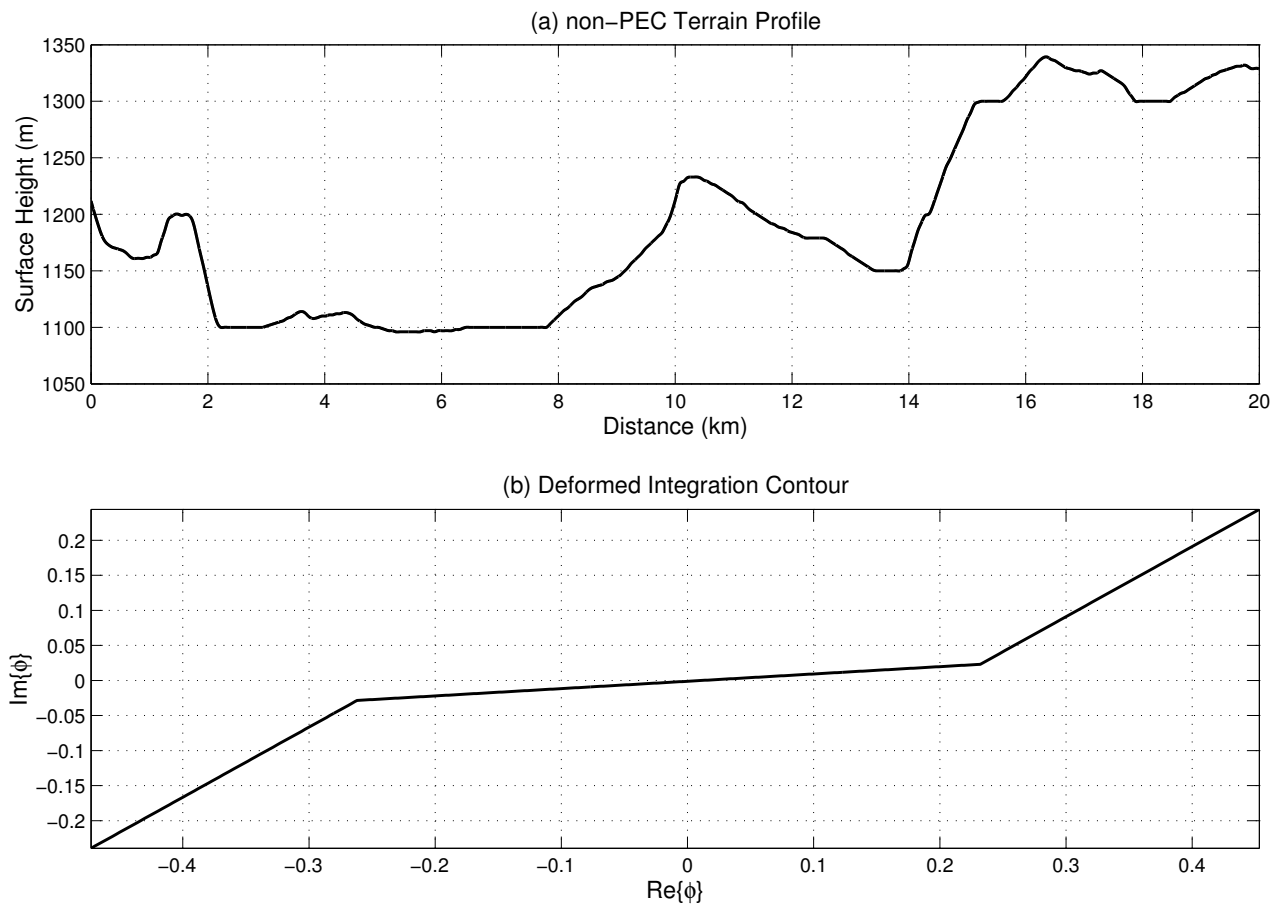

Figure 4.22: Deformed path of uphill profile at $500 \mathrm{MHz}$. 
The field strength predictions are evaluated using Free Space Propagation model, ITU-R recommendations; ITUR370, ITUR529, ITUR1546, and FCC Curves. Figures 4.23-4.27 show these comparisons over the downhill profile, while Figures 4.28-4.32 illustrate examinations over the uphill one; for the TM and TE polarization in the $f=500 \mathrm{MHz}$ case. 
(a) non-PEC Terrain Profile

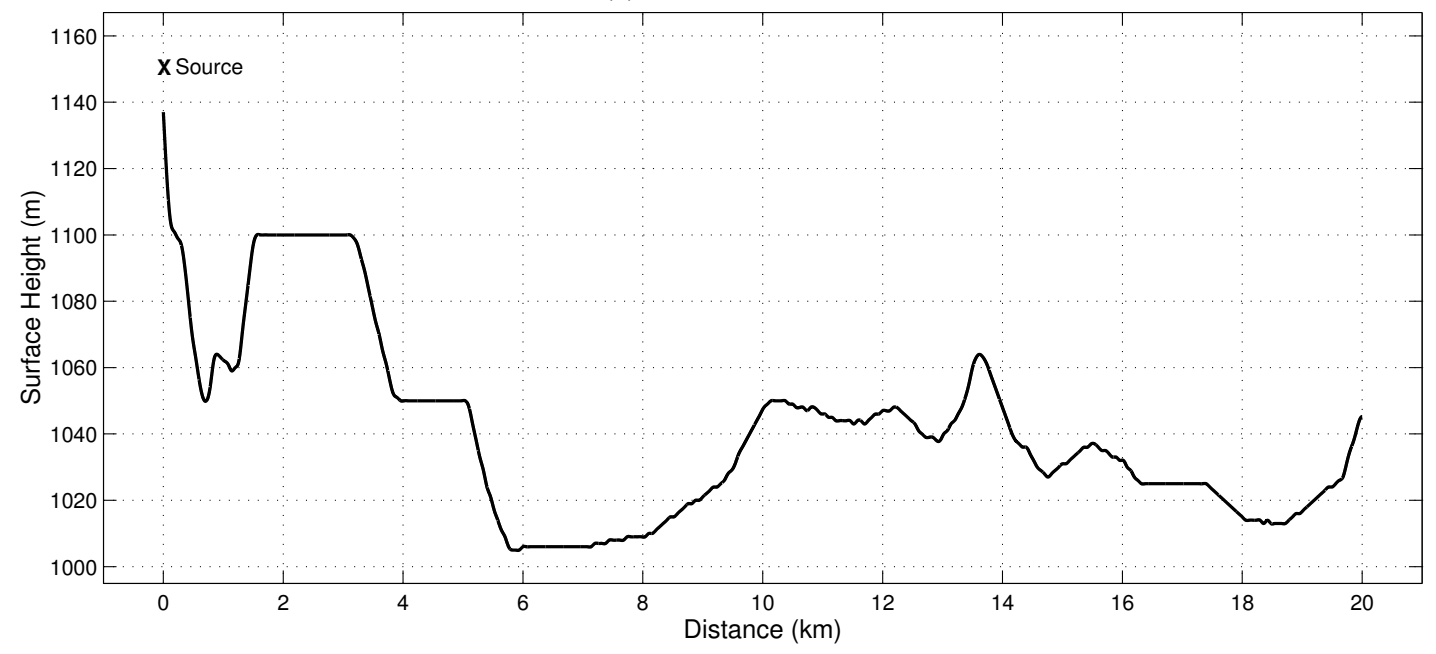

(b) TM Polarized Isotropic Radiator

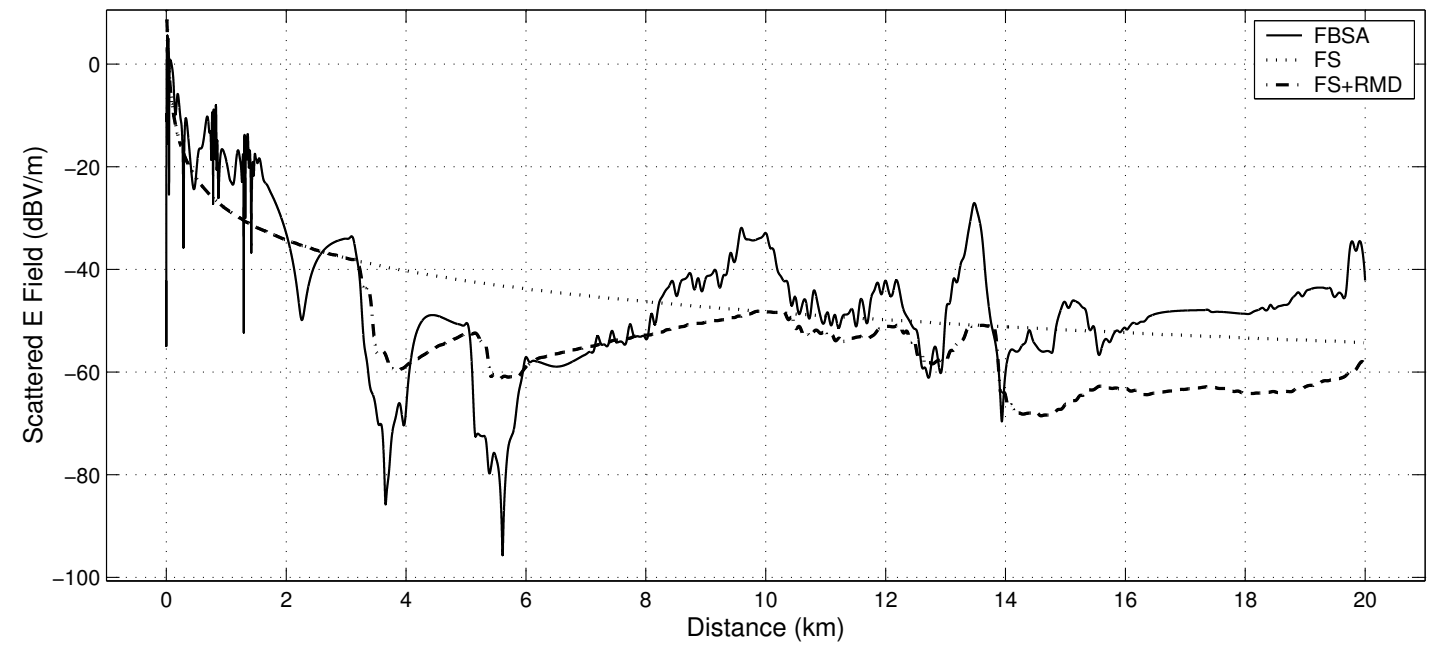

(c) TE Polarized Isotropic Radiator

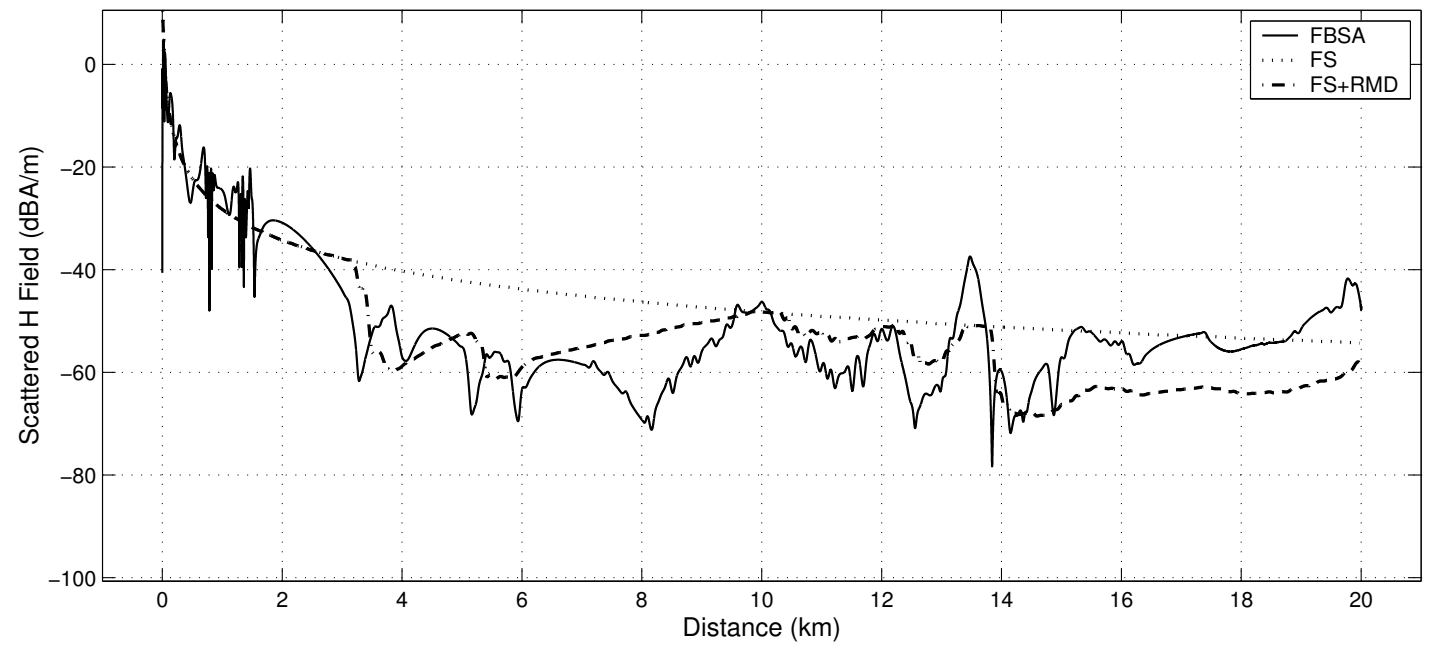

Figure 4.23: FreeSpace vs. IE over downhill terrain for $500 \mathrm{MHz}$. 
(a) non-PEC Terrain Profile

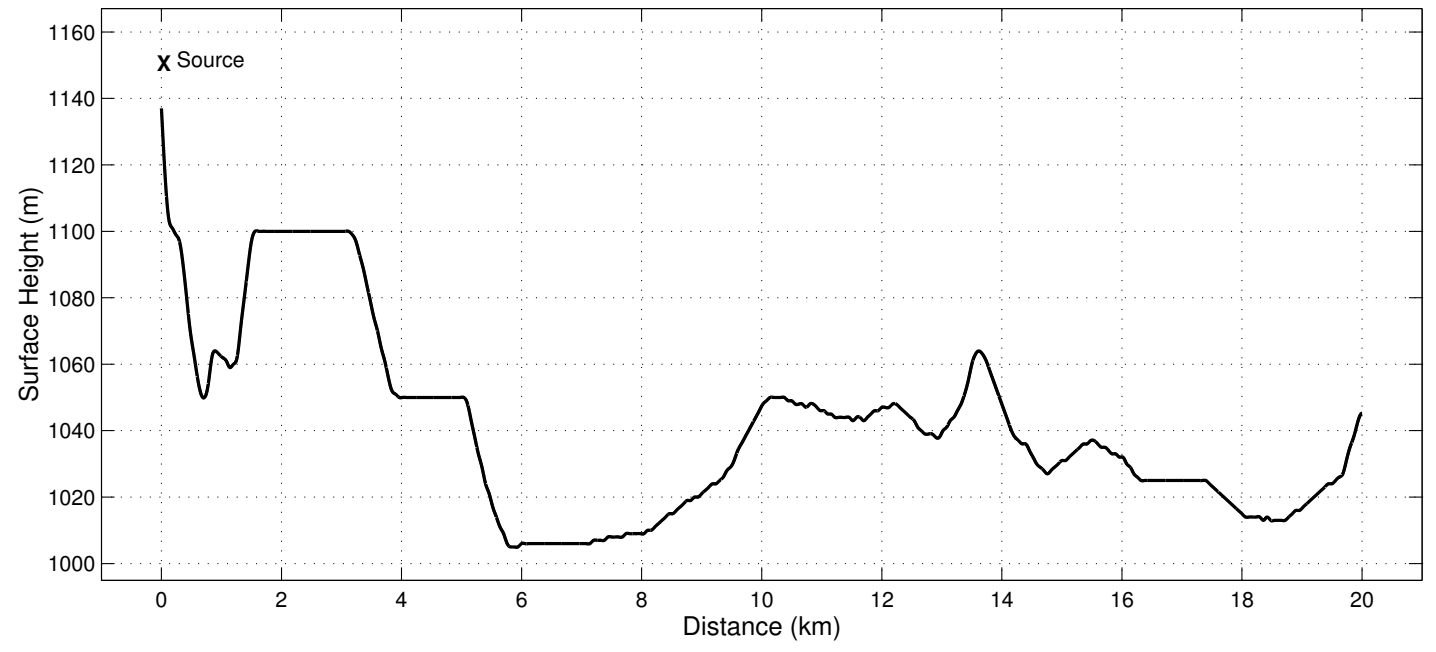

(b) TM Polarized Isotropic Radiator

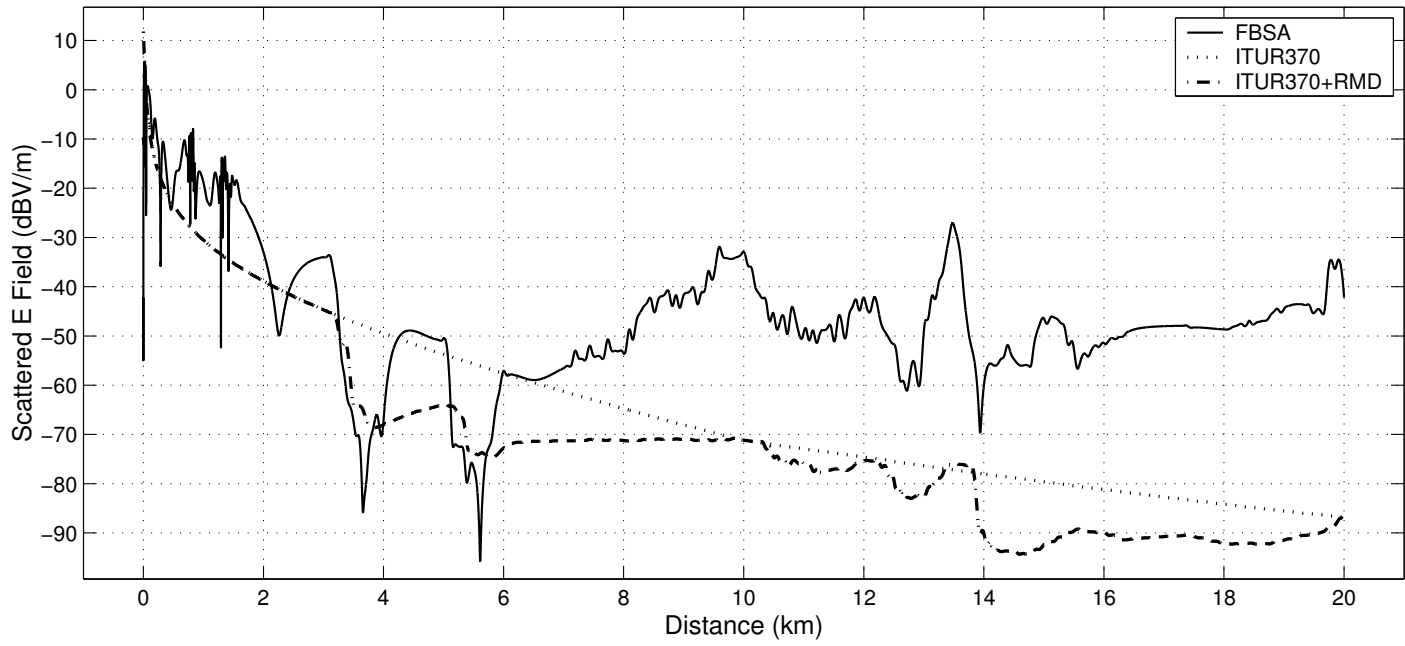

(c) TE Polarized Isotropic Radiator

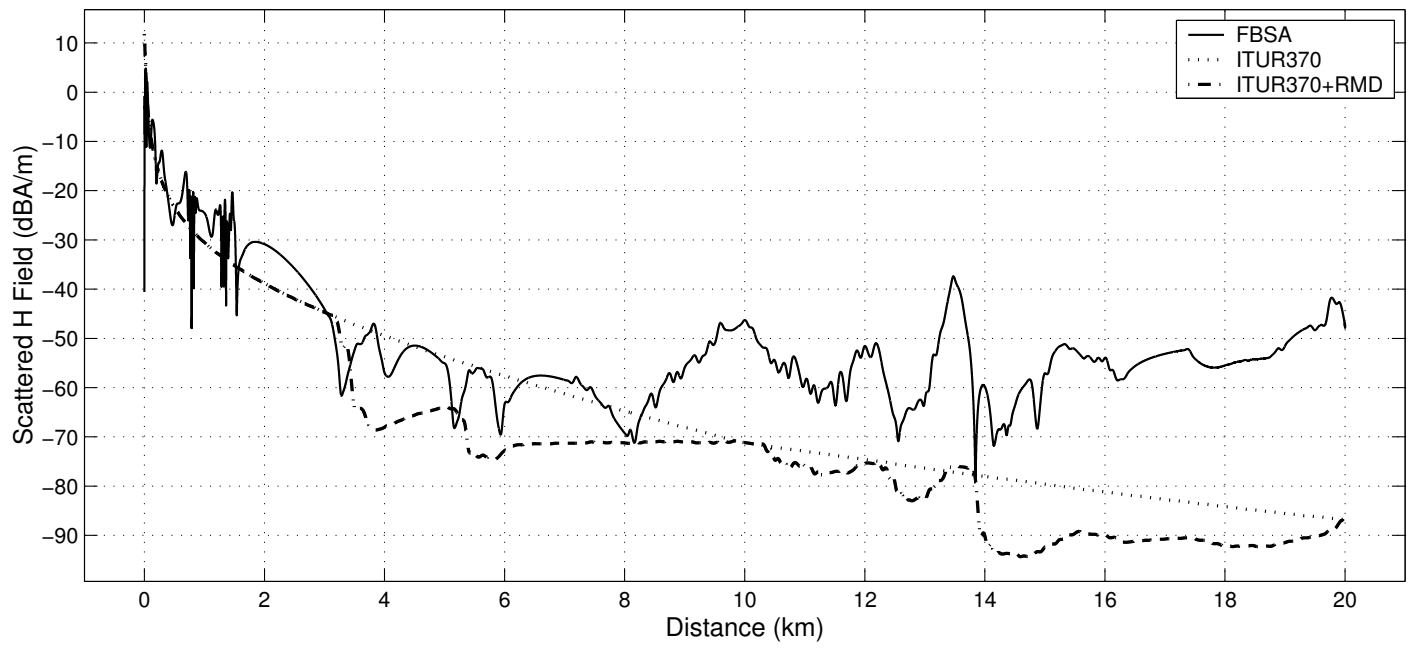

Figure 4.24: ITUR370 vs. IE over downhill terrain for $500 \mathrm{MHz}$. 
(a) non-PEC Terrain Profile

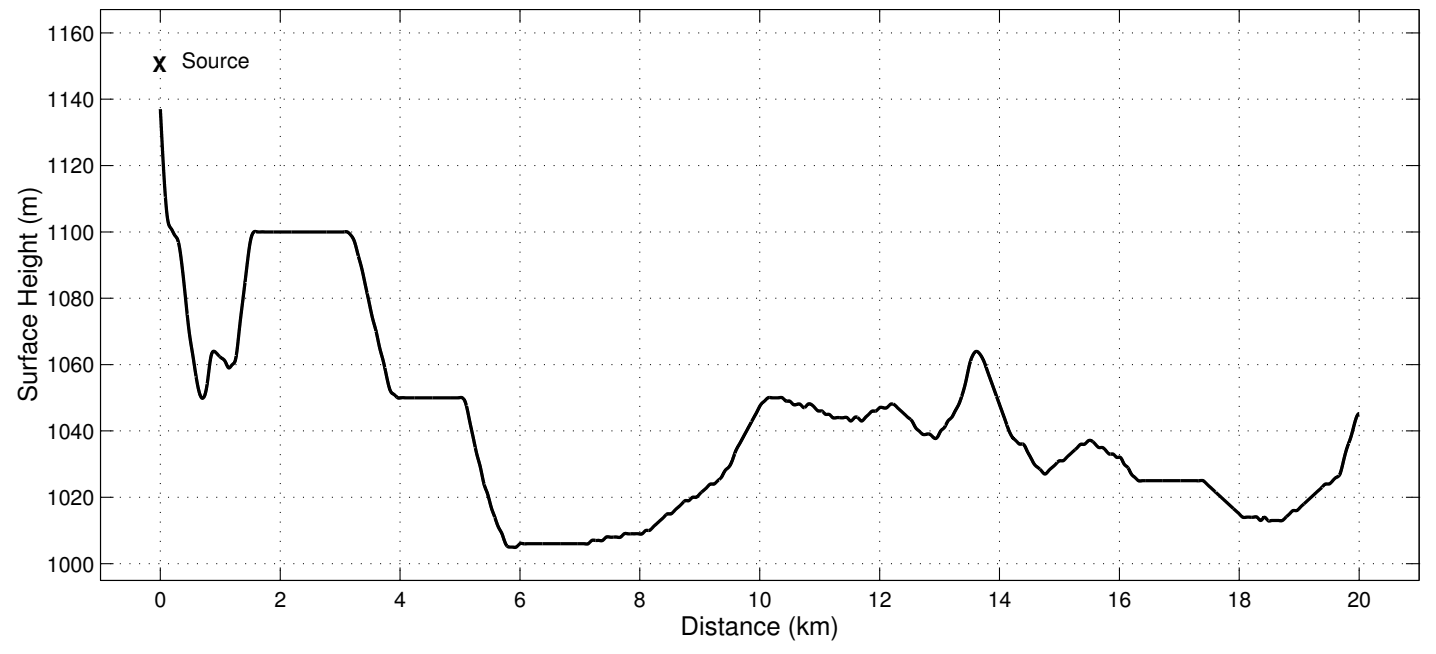

(b) TM Polarized Isotropic Radiator

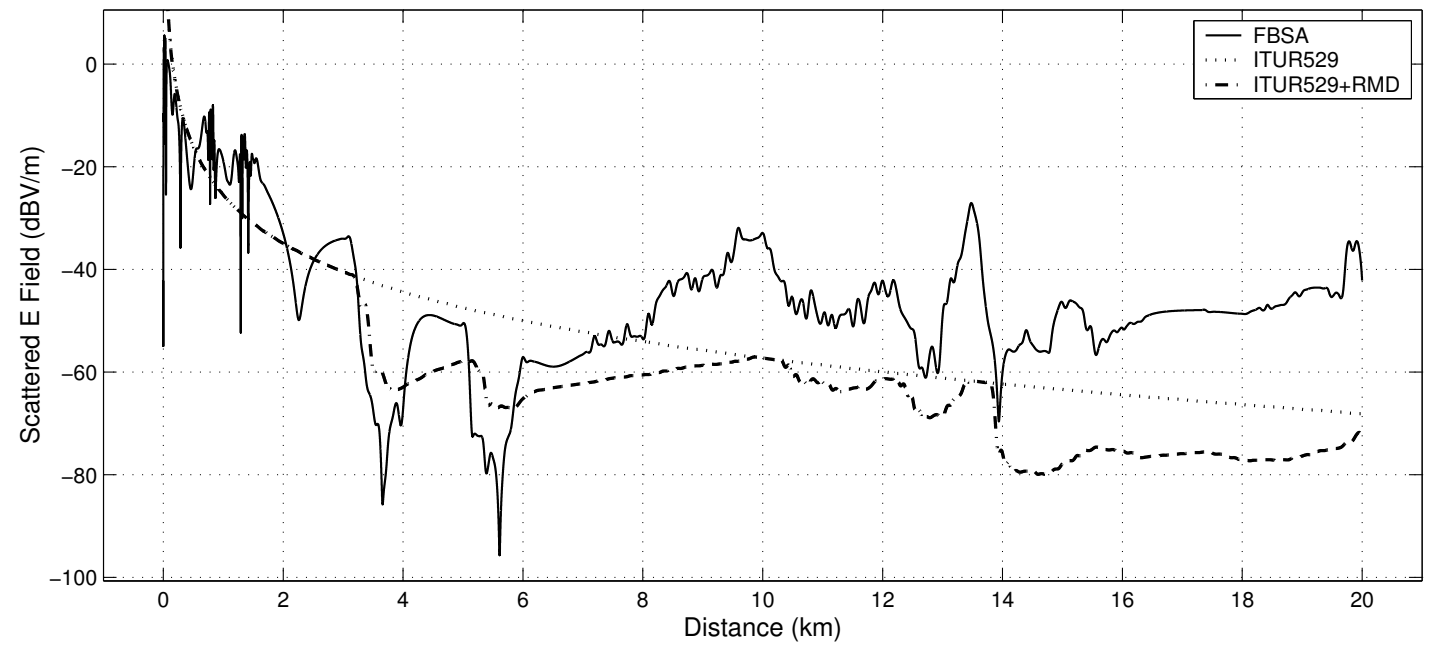

(c) TE Polarized Isotropic Radiator

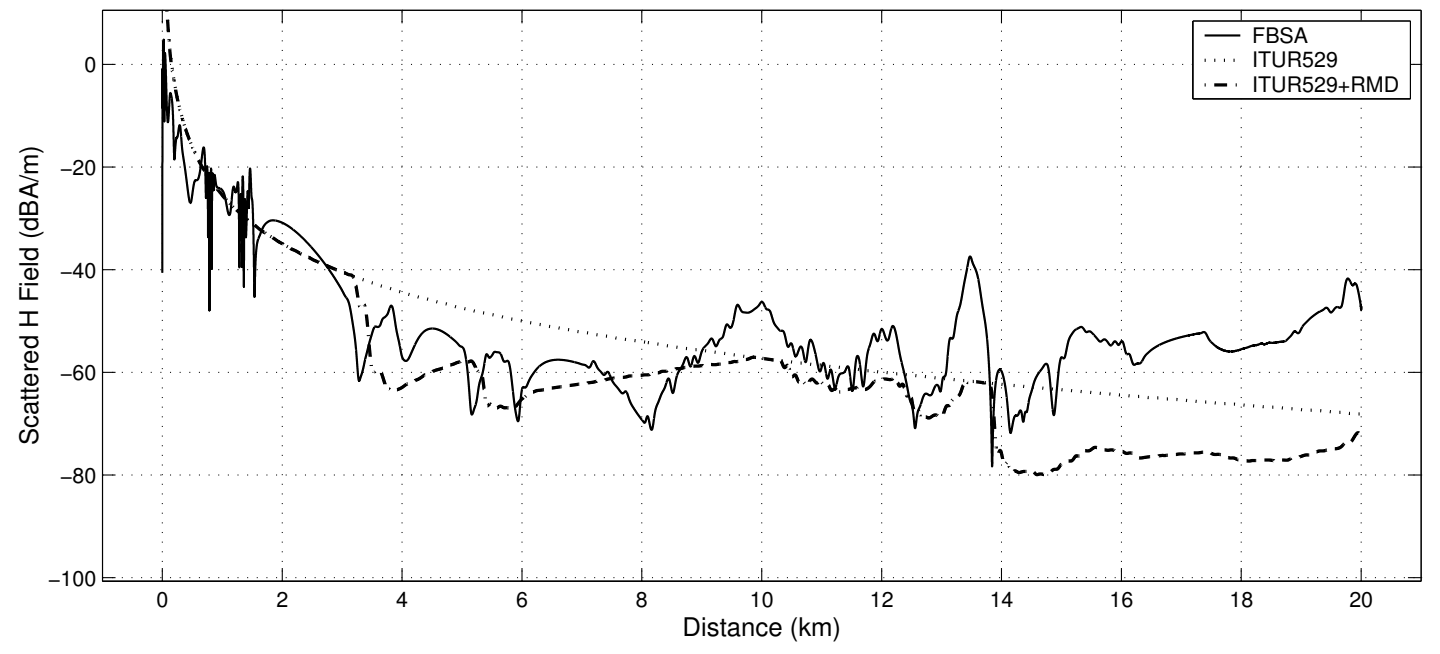

Figure 4.25: ITUR529 vs. IE over downhill terrain for $500 \mathrm{MHz}$. 
(a) non-PEC Terrain Profile

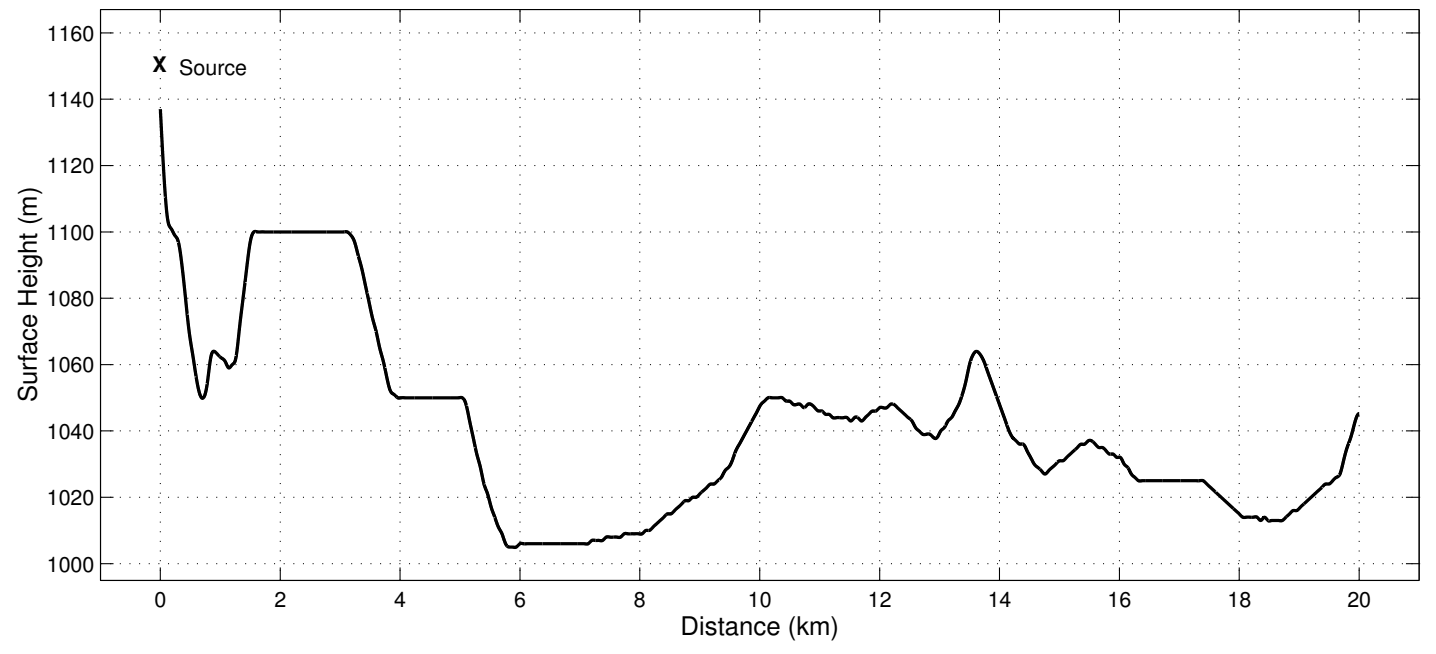

(b) TM Polarized Isotropic Radiator

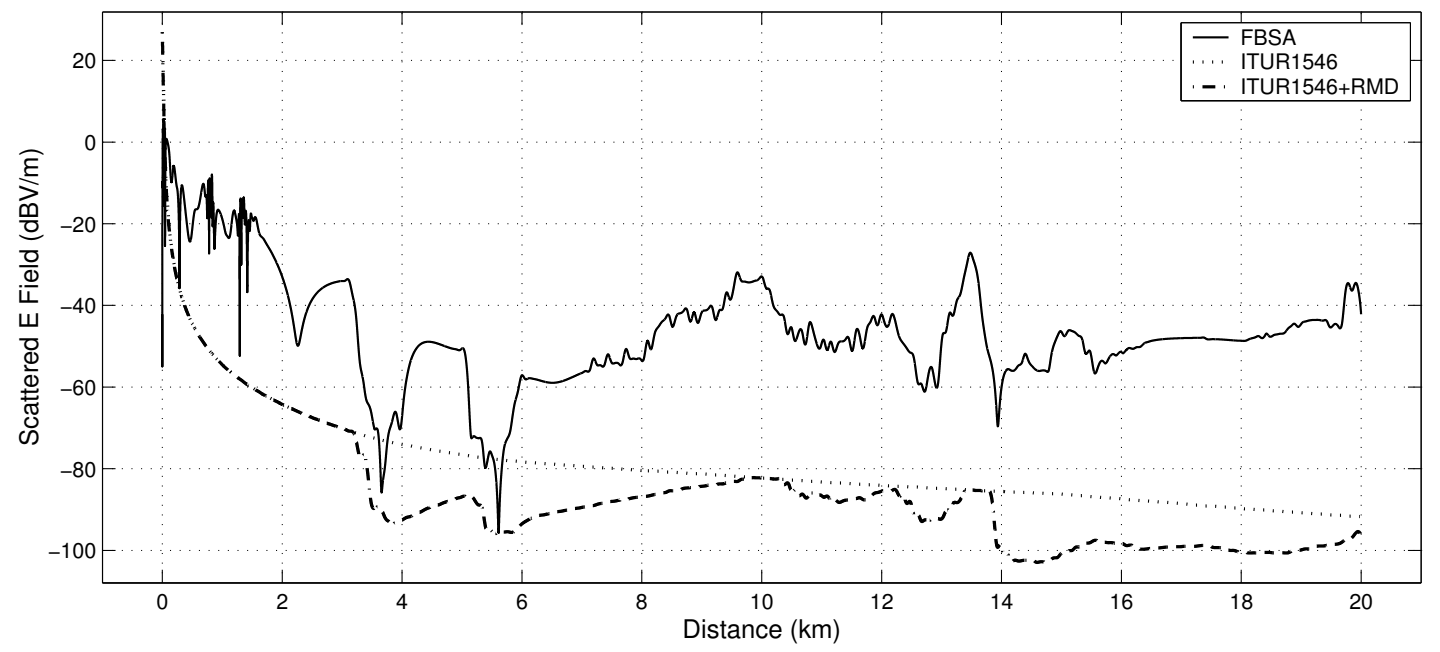

(c) TE Polarized Isotropic Radiator

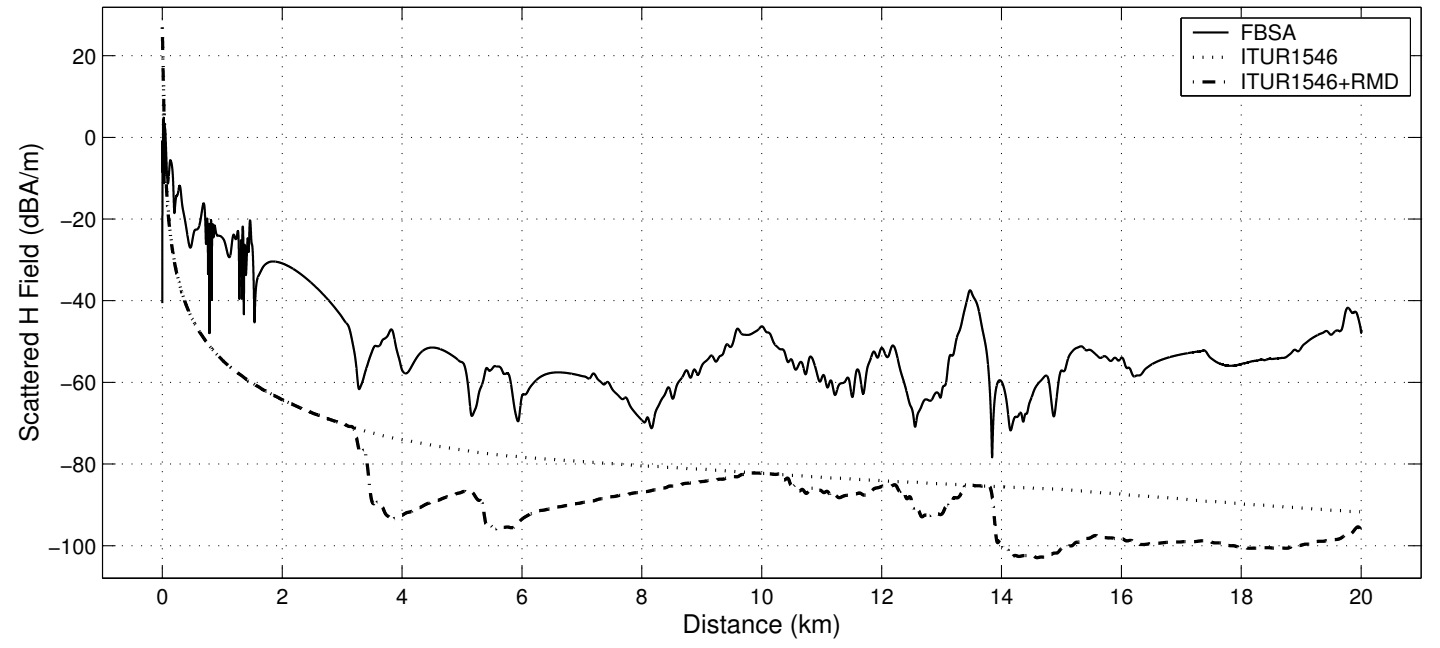

Figure 4.26: ITUR1546 vs. IE over downhill terrain for $500 \mathrm{MHz}$. 
(a) non-PEC Terrain Profile

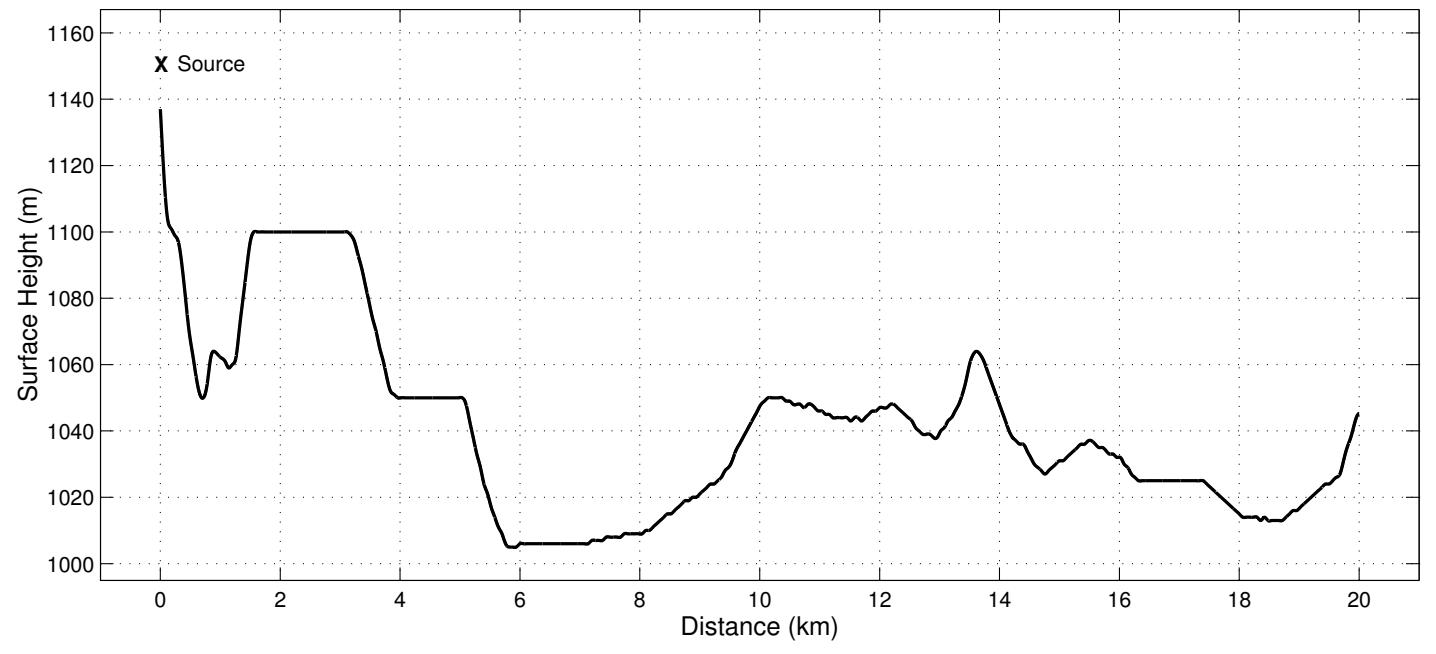

(b) TM Polarized Isotropic Radiator

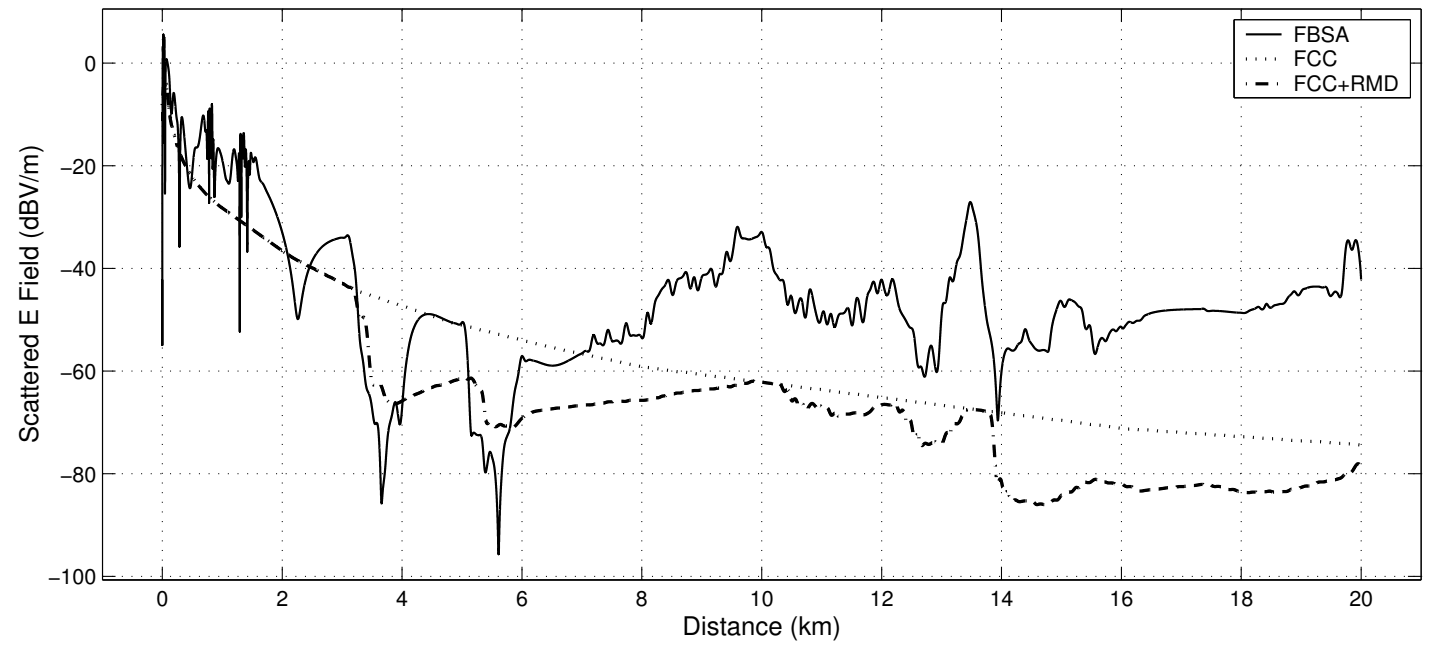

(c) TE Polarized Isotropic Radiator

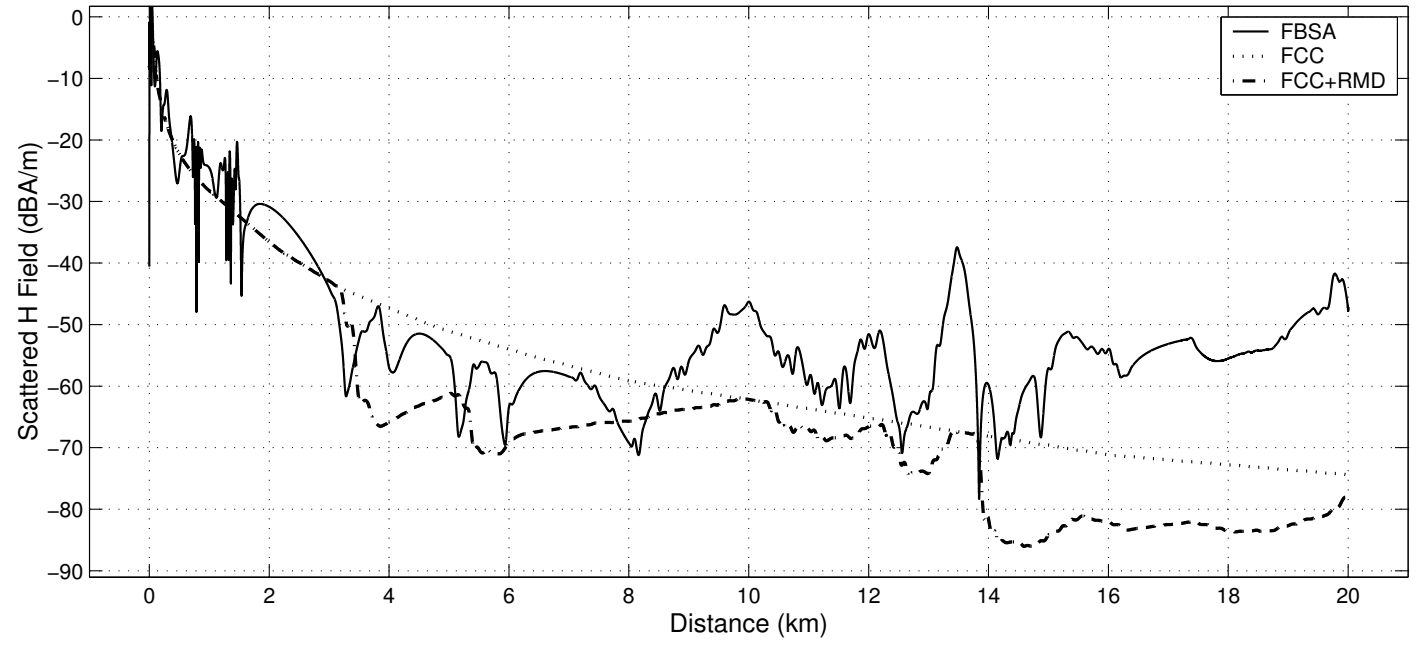

Figure 4.27: FCC vs. IE over downhill terrain for $500 \mathrm{MHz}$. 
(a) non-PEC Terrain Profile

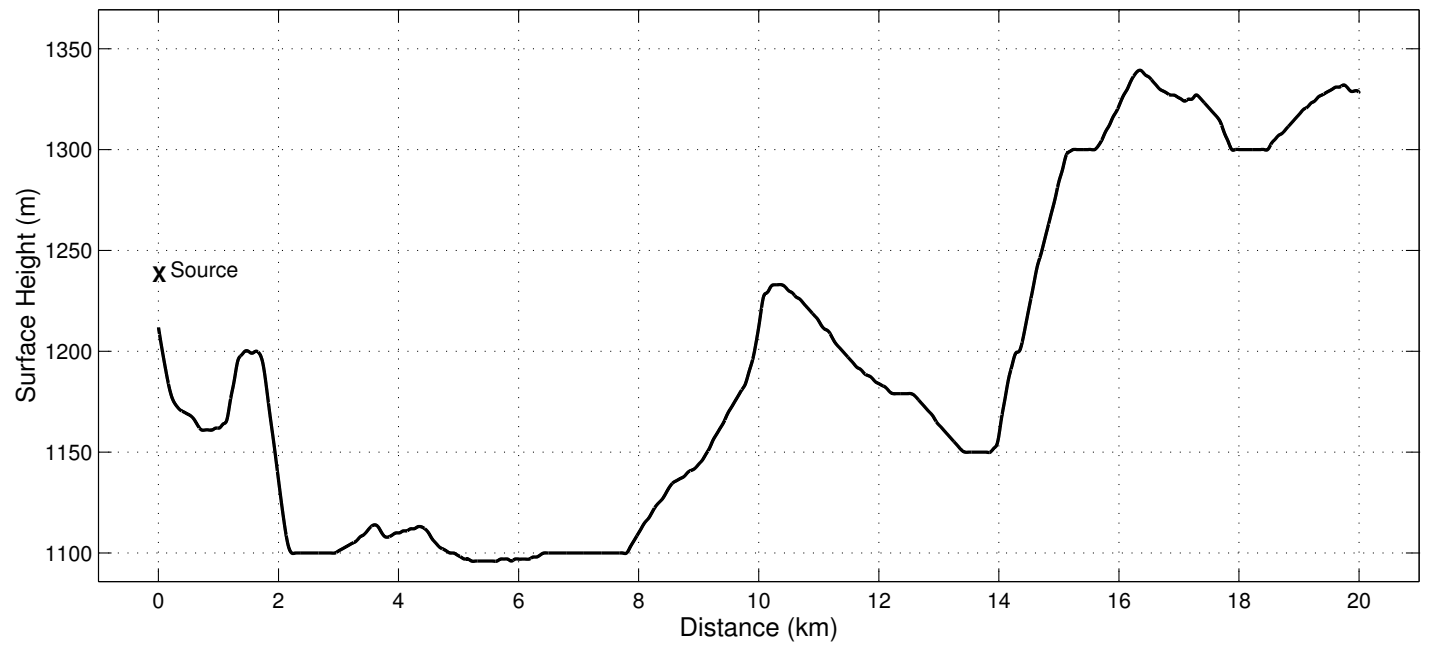

(b) TM Polarized Isotropic Radiator

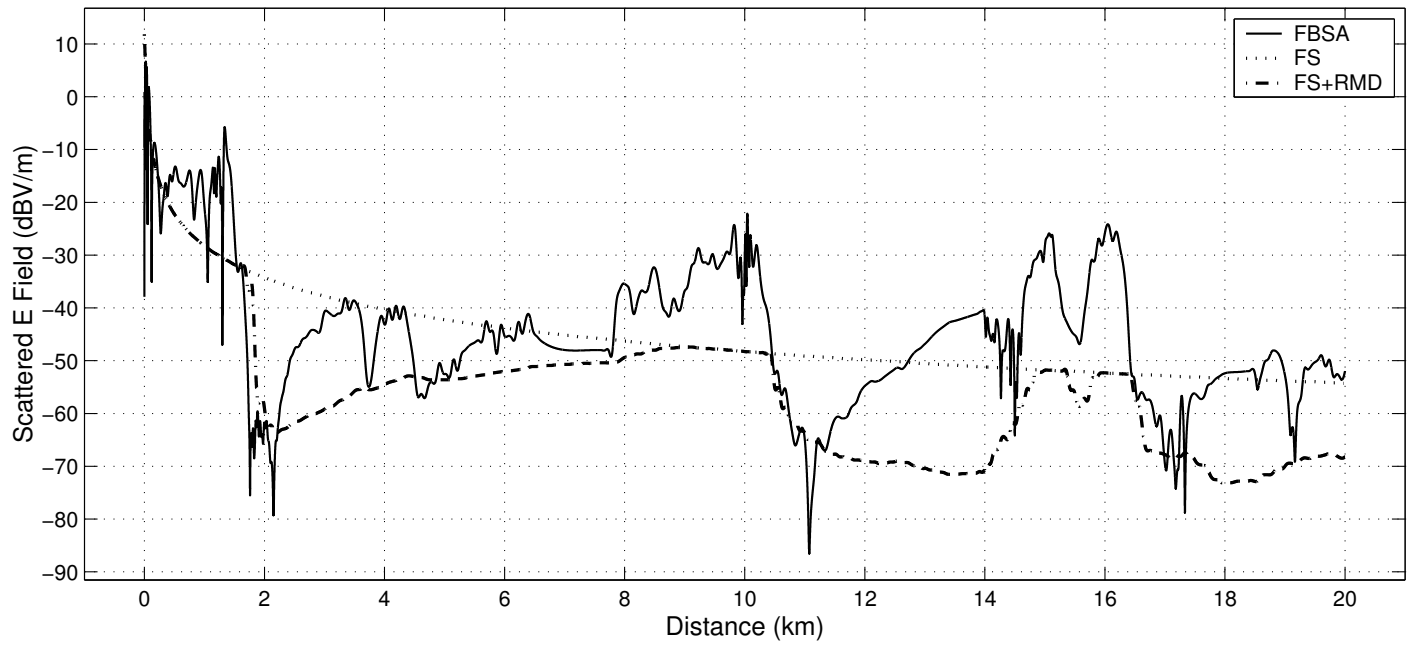

(c) TE Polarized Isotropic Radiator

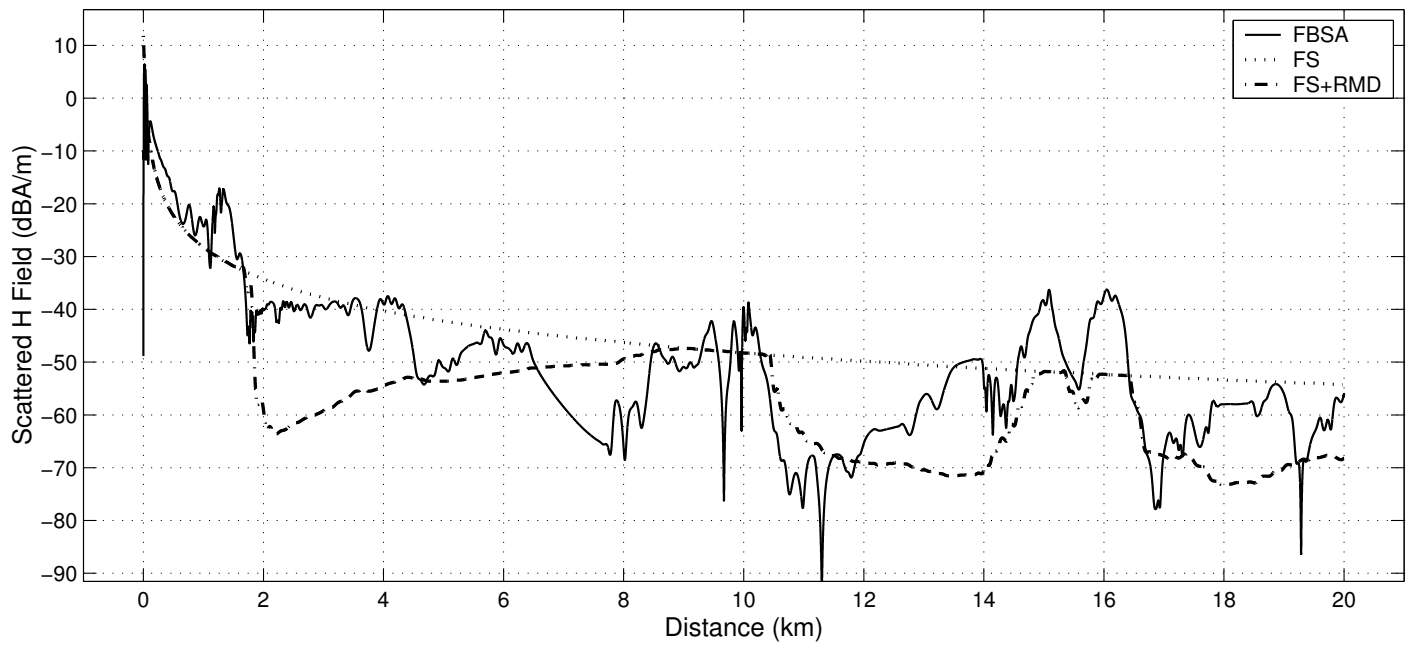

Figure 4.28: FreeSpace vs. IE over uphill terrain for $500 \mathrm{MHz}$. 
(a) non-PEC Terrain Profile

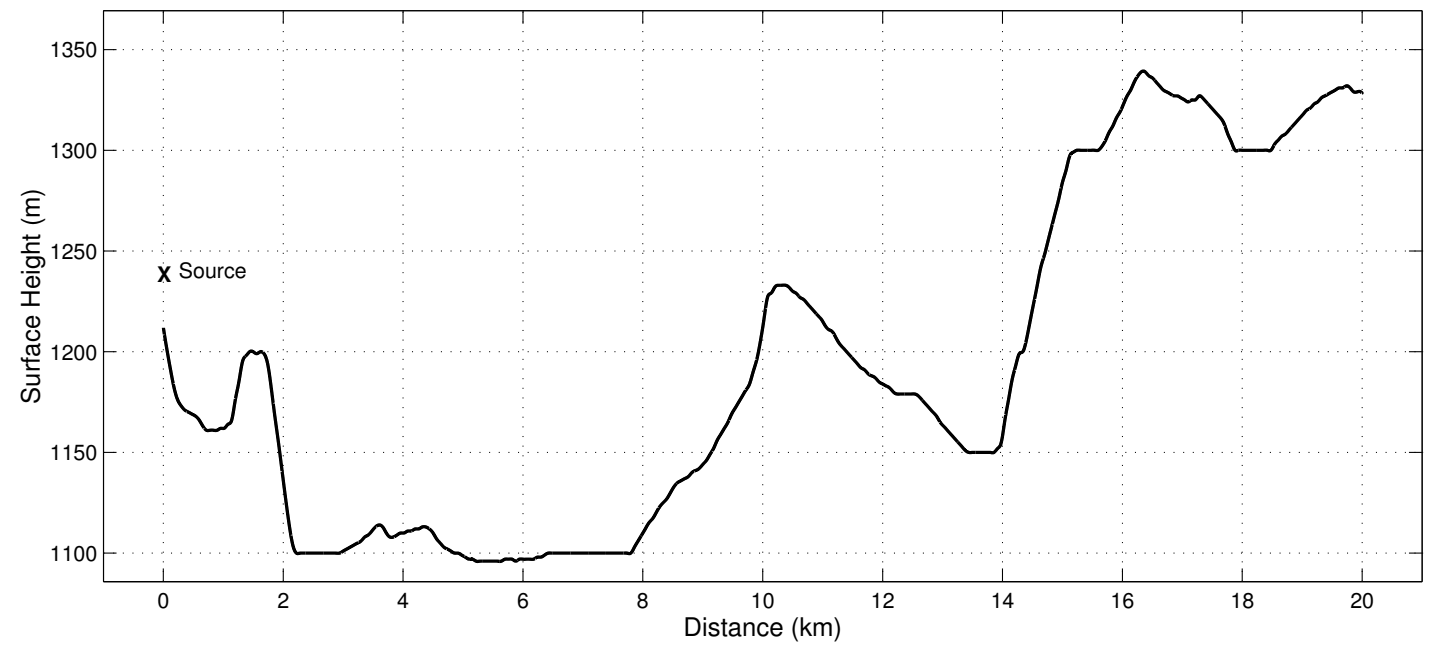

(b) TM Polarized Isotropic Radiator

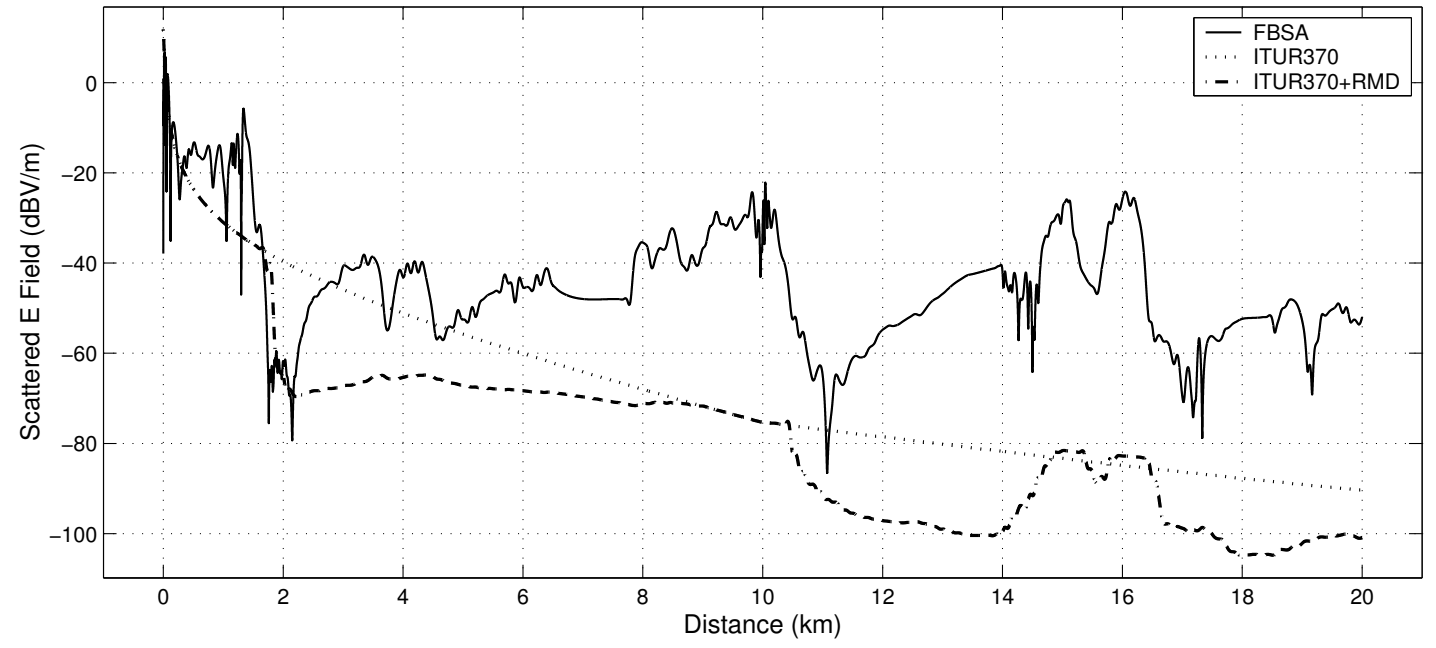

(c) TE Polarized Isotropic Radiator

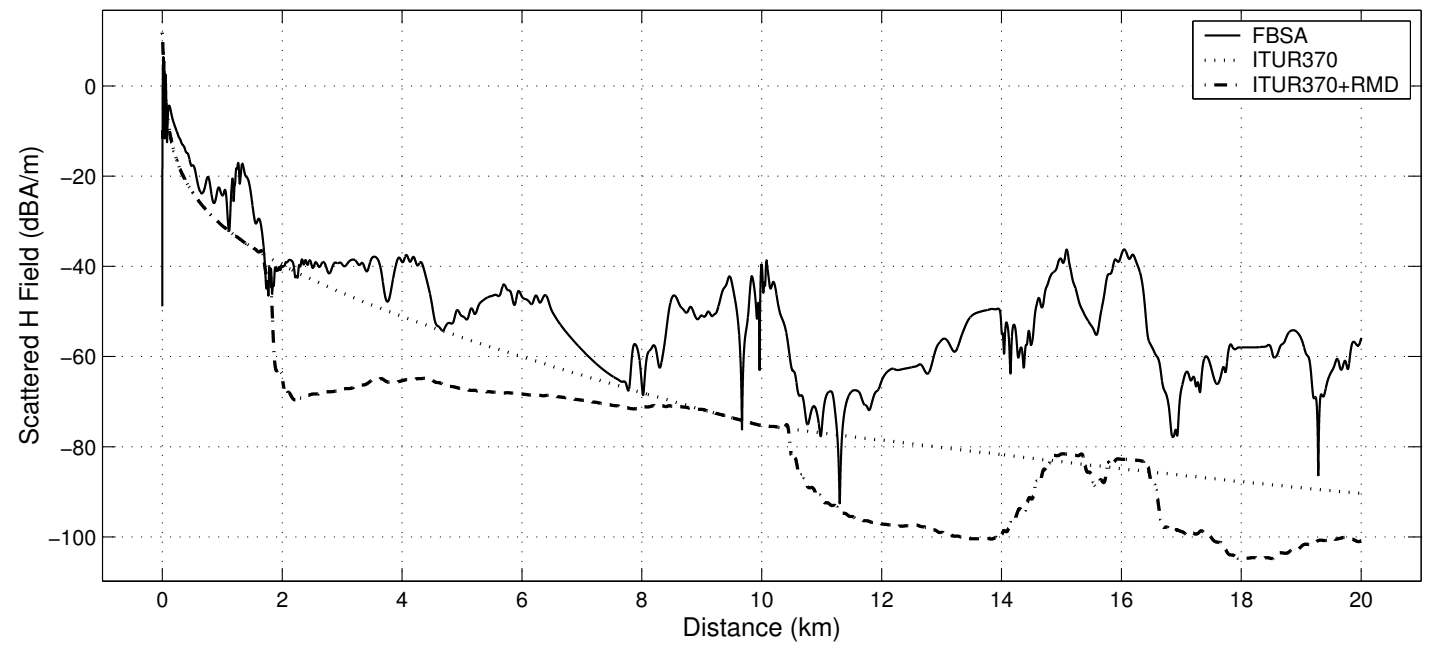

Figure 4.29: ITUR370 vs. IE over uphill terrain for $500 \mathrm{MHz}$. 
(a) non-PEC Terrain Profile

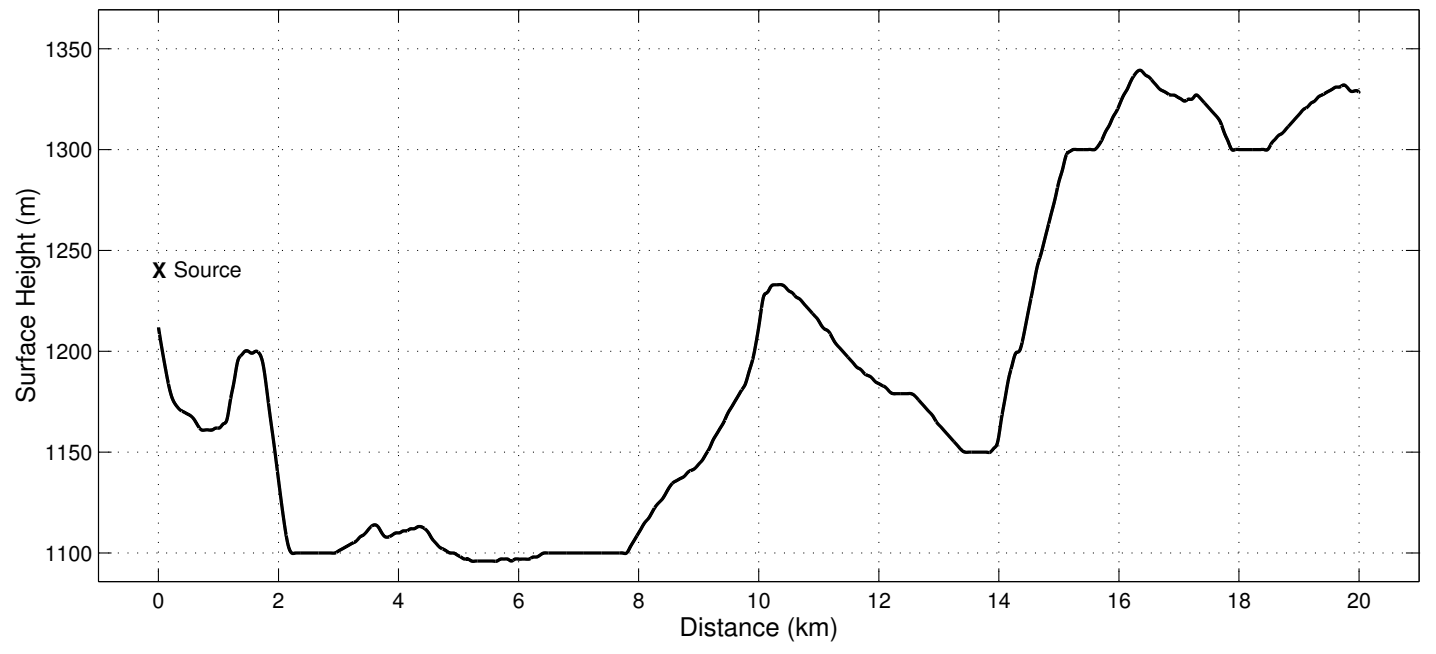

(b) TM Polarized Isotropic Radiator

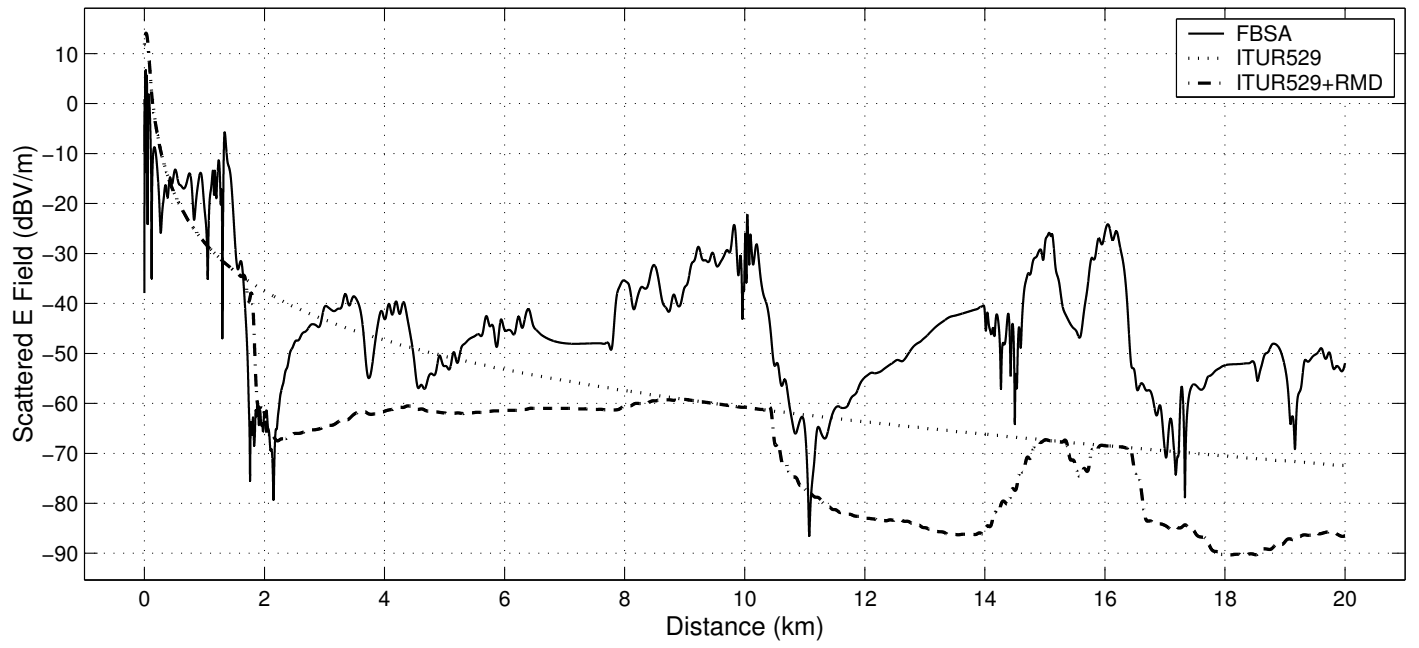

(c) TE Polarized Isotropic Radiator

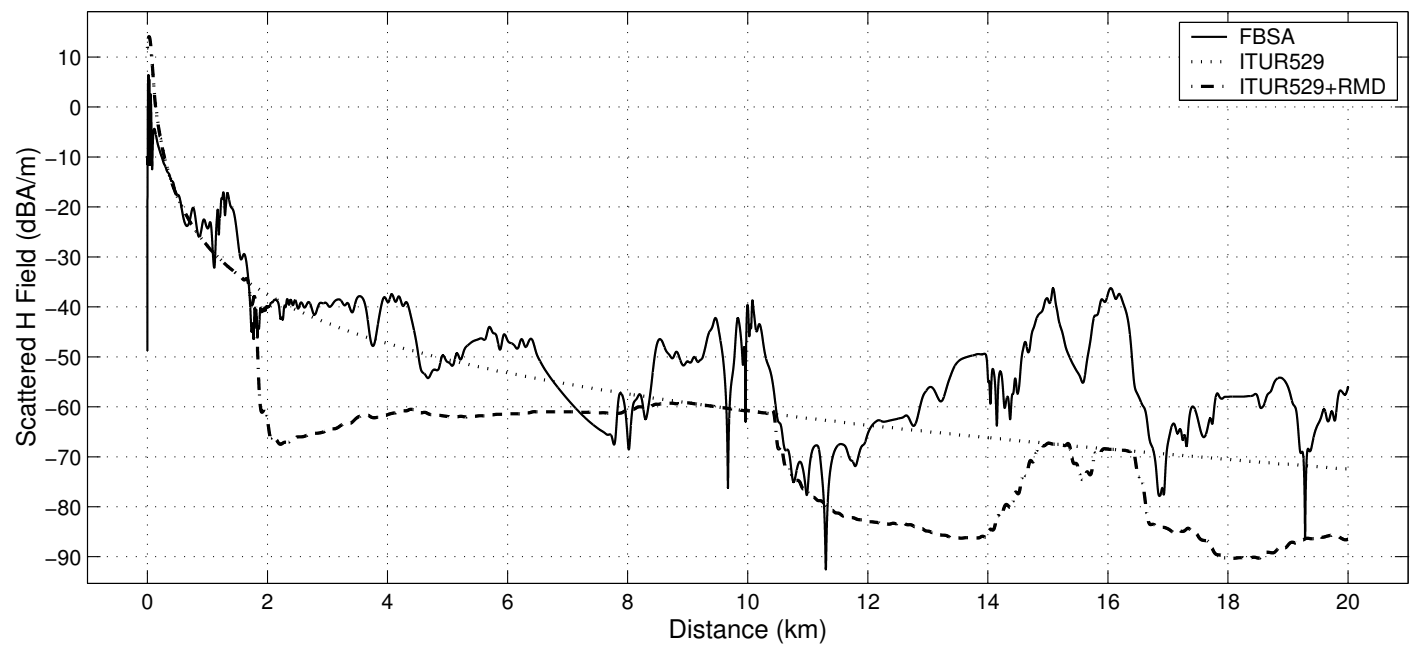

Figure 4.30: ITUR529 vs. IE over uphill terrain for $500 \mathrm{MHz}$. 
(a) non-PEC Terrain Profile

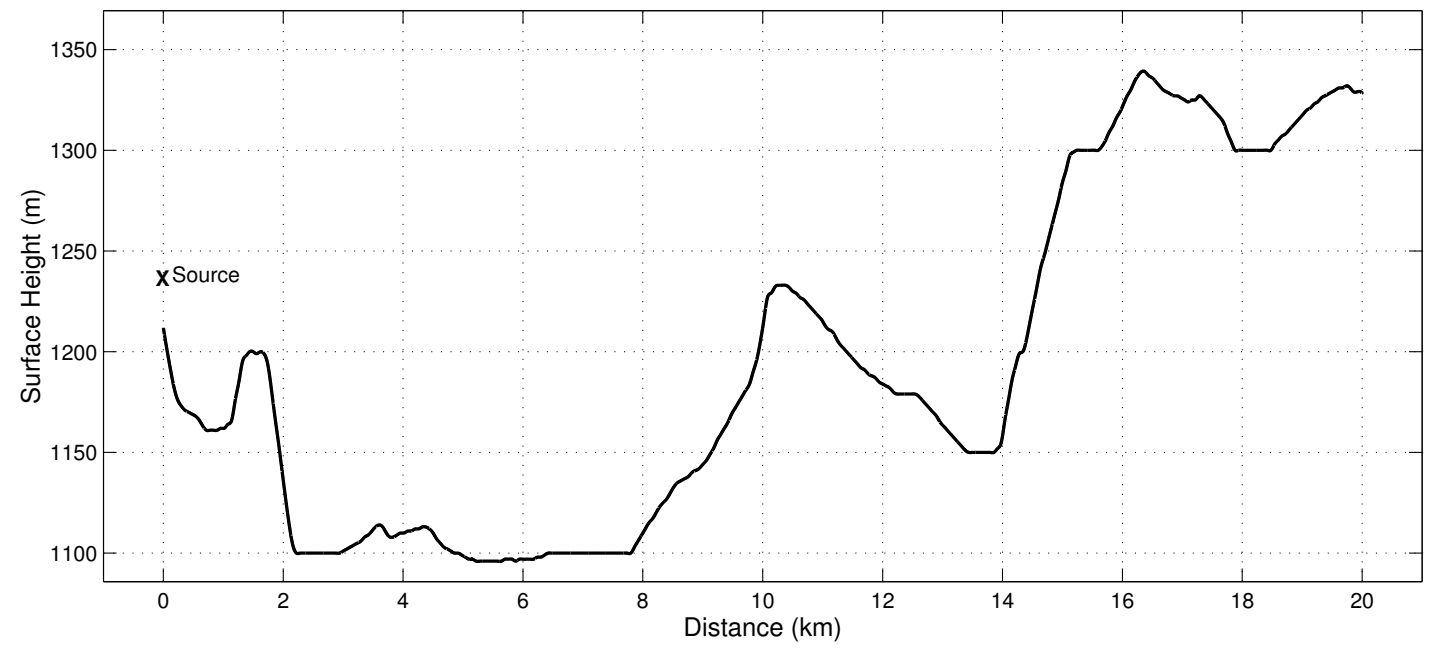

(b) TM Polarized Isotropic Radiator

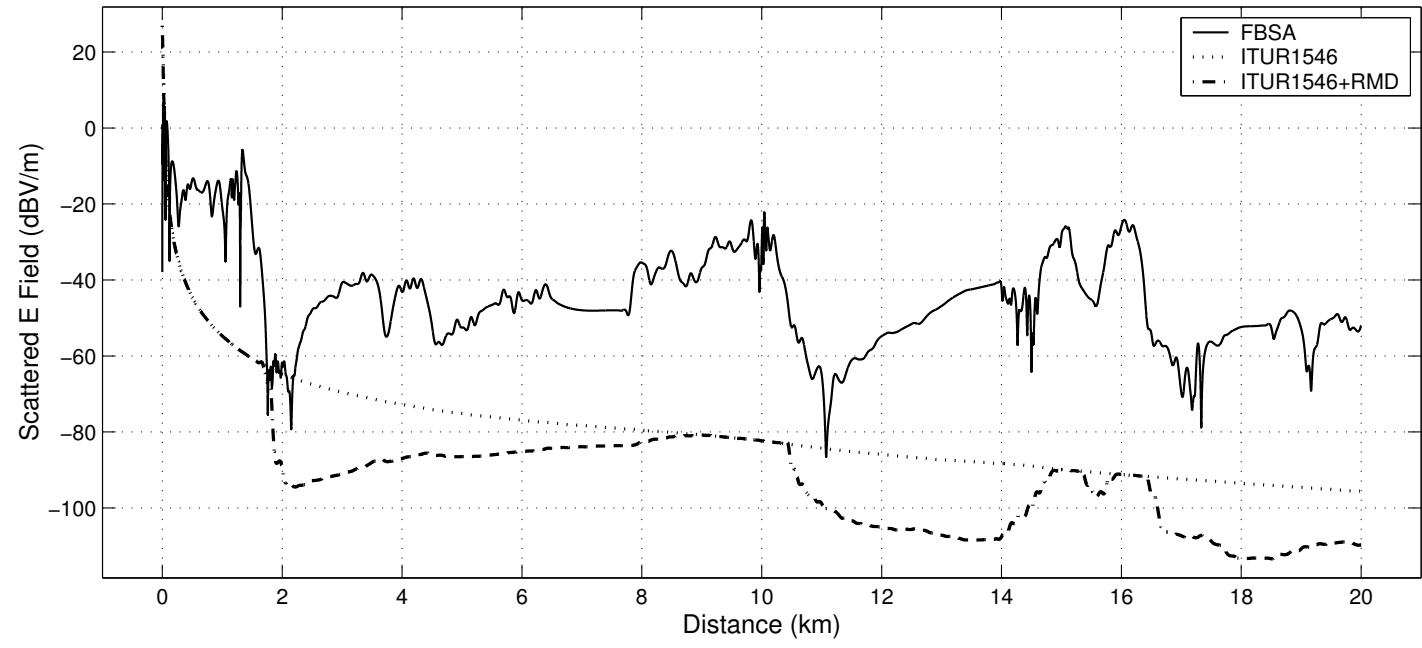

(c) TE Polarized Isotropic Radiator

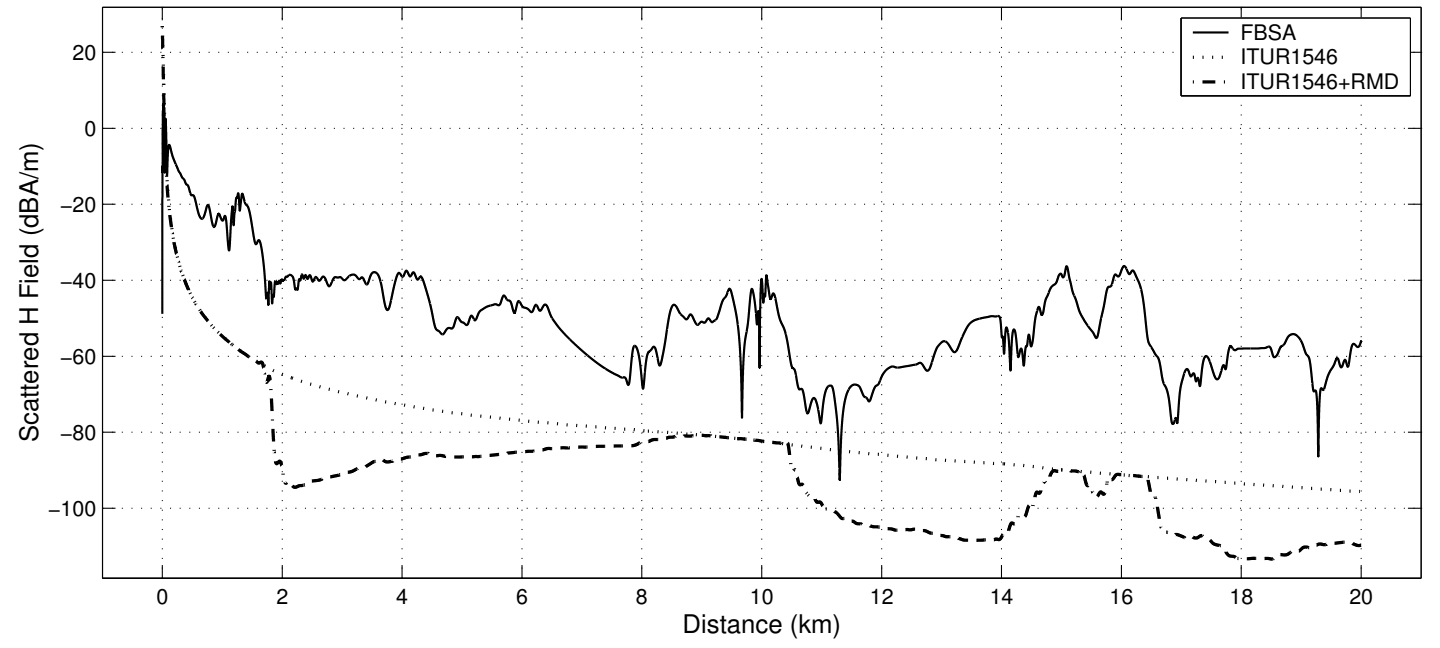

Figure 4.31: ITUR1546 vs. IE over uphill terrain for $500 \mathrm{MHz}$. 
(a) non-PEC Terrain Profile

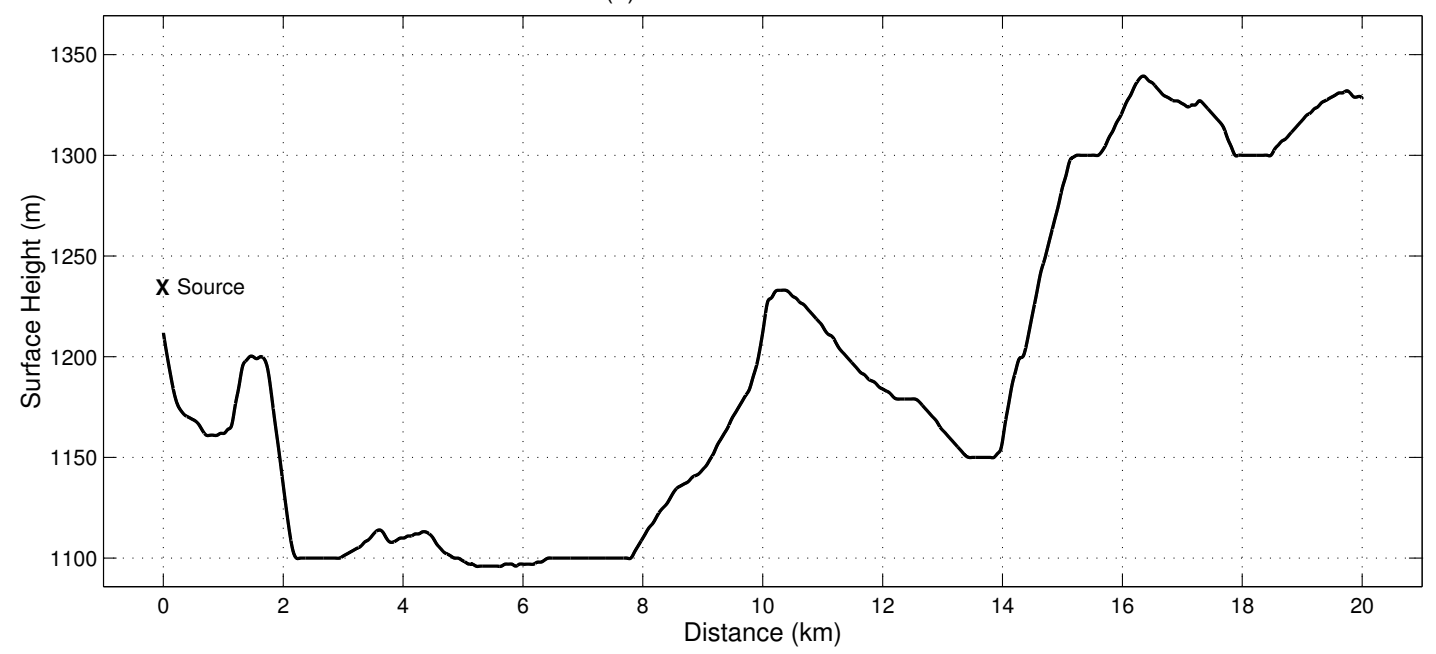

(b) TM Polarized Isotropic Radiator

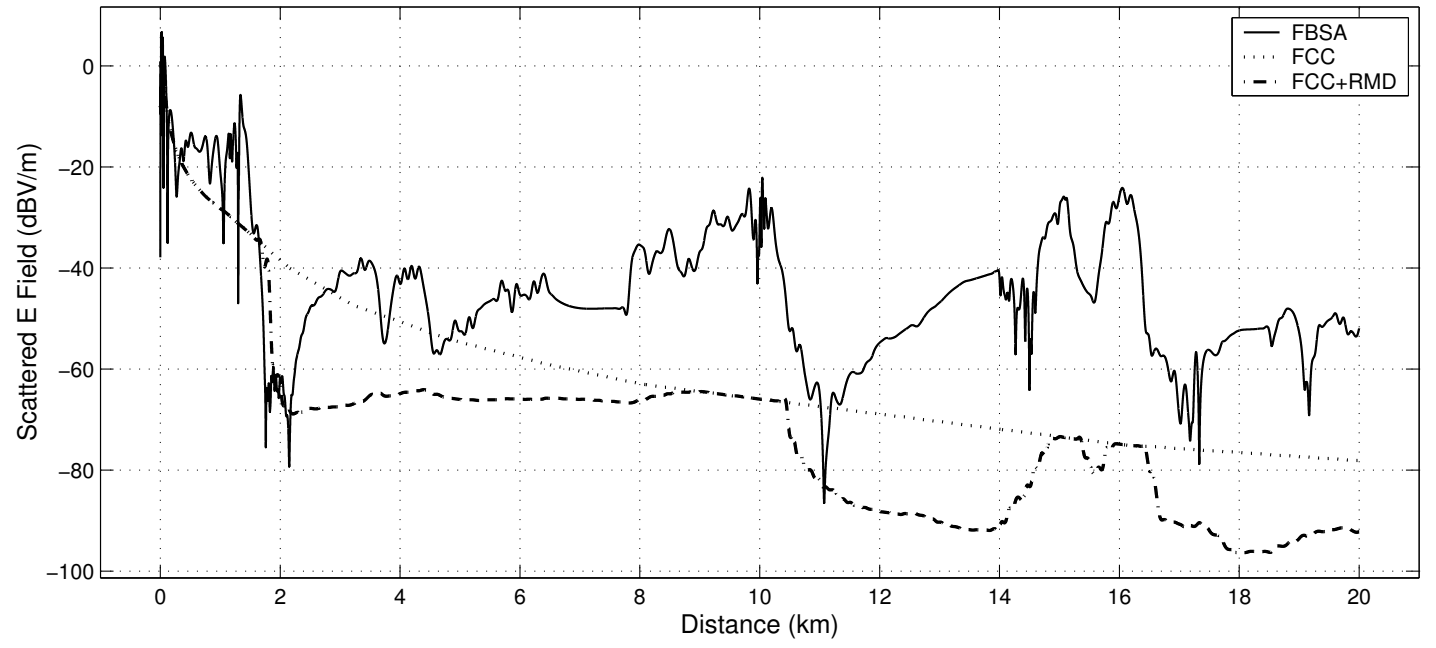

(c) TE Polarized Isotropic Radiator

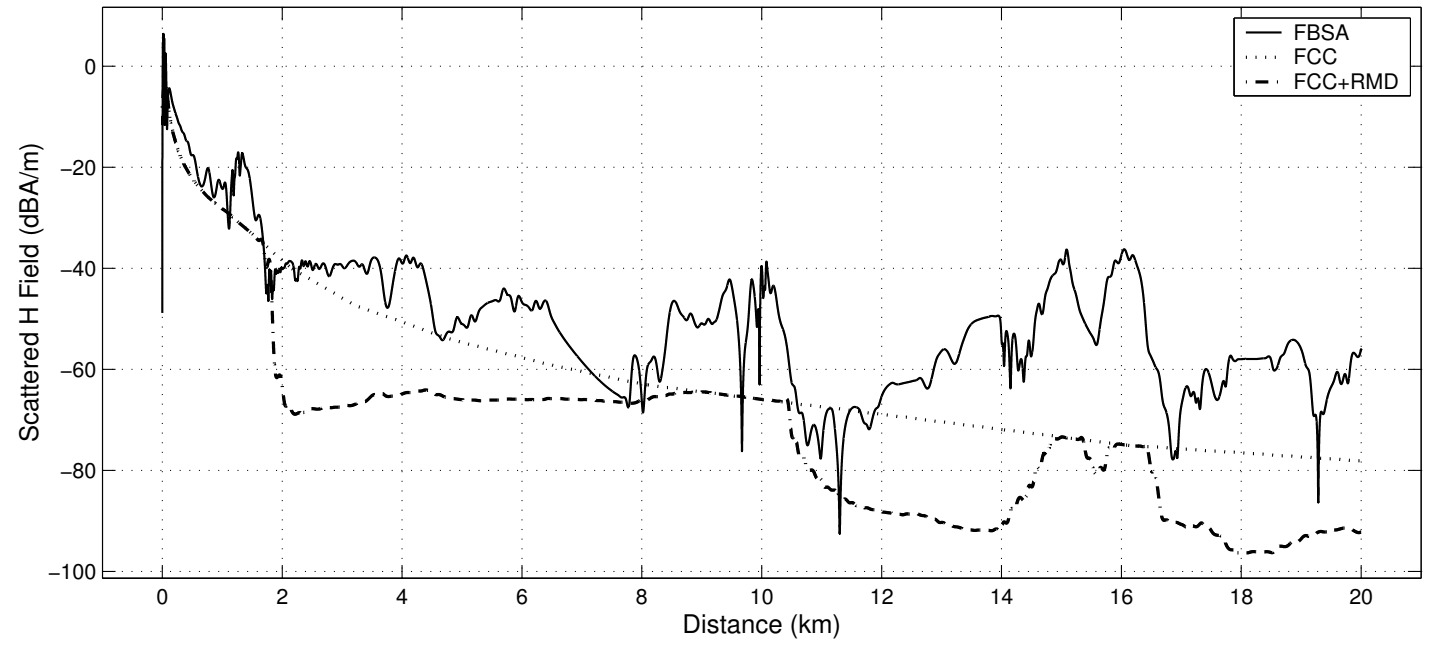

Figure 4.32: FCC vs. IE over uphill terrain for $500 \mathrm{MHz}$. 
Table 4.4 shows the absolute error values for different propagation models and polarizations over uphill and downhill profiles for $500 \mathrm{MHz}$.

\begin{tabular}{|c|c|c|c|c|}
\hline Absolute Error (\%) & DH (TM) & DH (TE) & UH (TM) & UH (TE) \\
\hline FreeSpace & 24.49 & 19.35 & 24.87 & 19.59 \\
\hline ITUR-370 & 53.25 & 35.83 & 62.84 & 40.45 \\
\hline ITUR-529 & 31.81 & 18.57 & 39.32 & 21.10 \\
\hline ITUR-1546 & 71.90 & 53.53 & 80.81 & 58.47 \\
\hline FCC & 38.73 & 23.10 & 47.90 & 27.22 \\
\hline FreeSpace + EpsPet & 23.73 & 14.62 & 31.66 & 20.83 \\
\hline ITUR-370 + EpsPet & 61.91 & 44.92 & 79.04 & 56.91 \\
\hline ITUR-529 + EpsPet & 39.36 & 25.67 & 55.78 & 37.73 \\
\hline ITUR-1546 + EpsPet & 85.52 & 67.14 & 101.98 & 78.77 \\
\hline FCC + EpsPet & 21.89 & 26.27 & 36.06 & 33.35 \\
\hline
\end{tabular}

Table 4.4: Absolute error values for $500 \mathrm{MHz}$

It is observed that for an isotropic source having $500 \mathrm{MHz}$ operating frequency, without RMD corrections, using FCC curves, free space propagation or ITUR-529 model is preferable than the others over both uphill and downhill profiles in TE and TM polarization cases. However, free space propagation loss with multiple diffraction is the most preferable model among others. Note that, modified Hata method ITUR-529 model gives the original Hata results for $d<20 \mathrm{~km}$, and the worst model seems to be the ITUR-1546, again.

Also, it is worth mentioning that, the empirical propagation models and multiple diffraction corrections do not respond to changes in electrical properties of terrain such as the relative permittivity and conductivity.

The last frequency test is implemented for $f=890 \mathrm{MHz}$ case. Figure 4.33 (b) and 4.34 (b) illustrate the deformed integration paths of downhill and uphill profiles, respectively, for $890 \mathrm{MHz}$. Required parameters for the spectral acceleration are included in Table 4.5 for this case.

Profiles are considered to be impedance surfaces of $\eta_{s}=25+j 20 \Omega$. At 890 $\mathrm{MHz}$, this impedance corresponds to a relative permittivity of $\epsilon_{r}=30.43$ and a conductivity of $\sigma=6.689 \mathrm{~S} / \mathrm{m}$. 


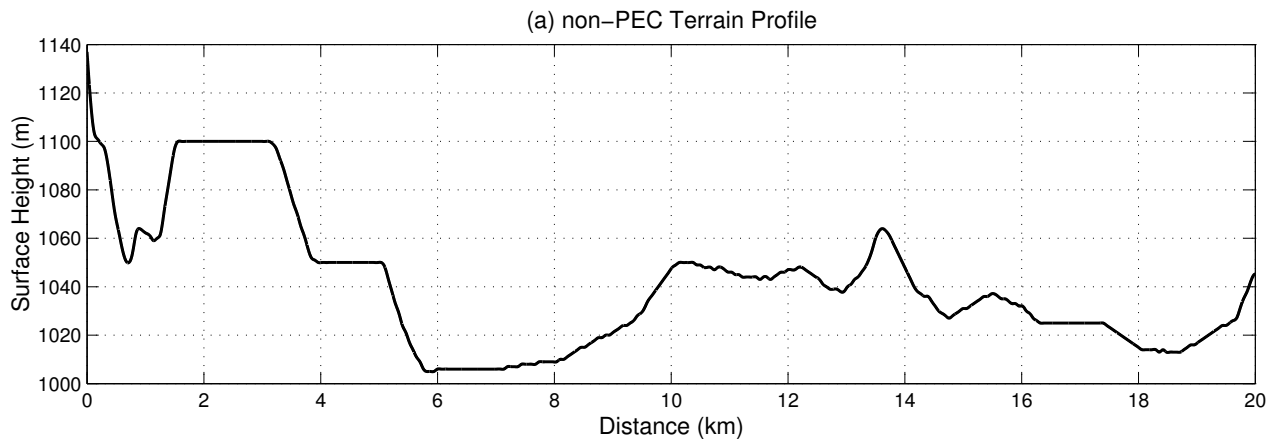

(b) Deformed Integration Contour

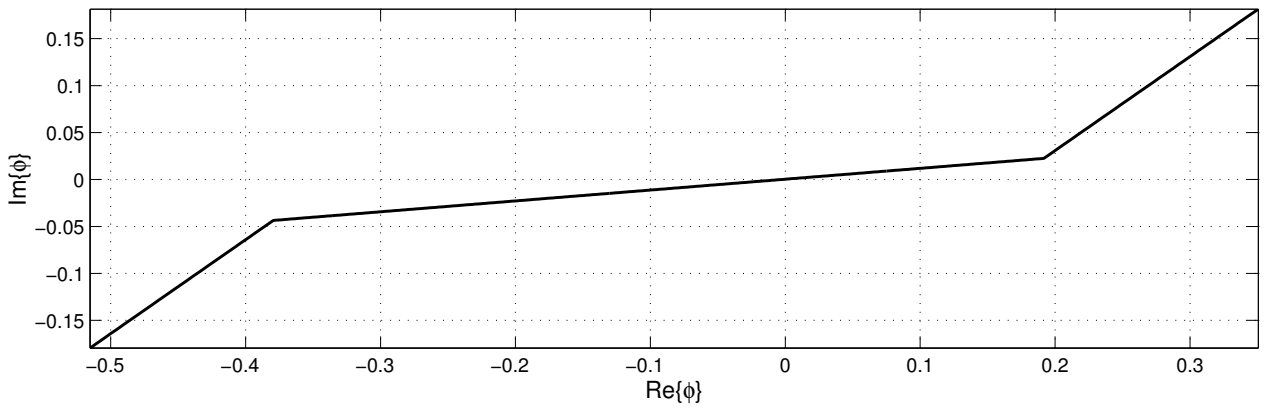

Figure 4.33: Deformed path of downhill profile at $890 \mathrm{MHz}$.
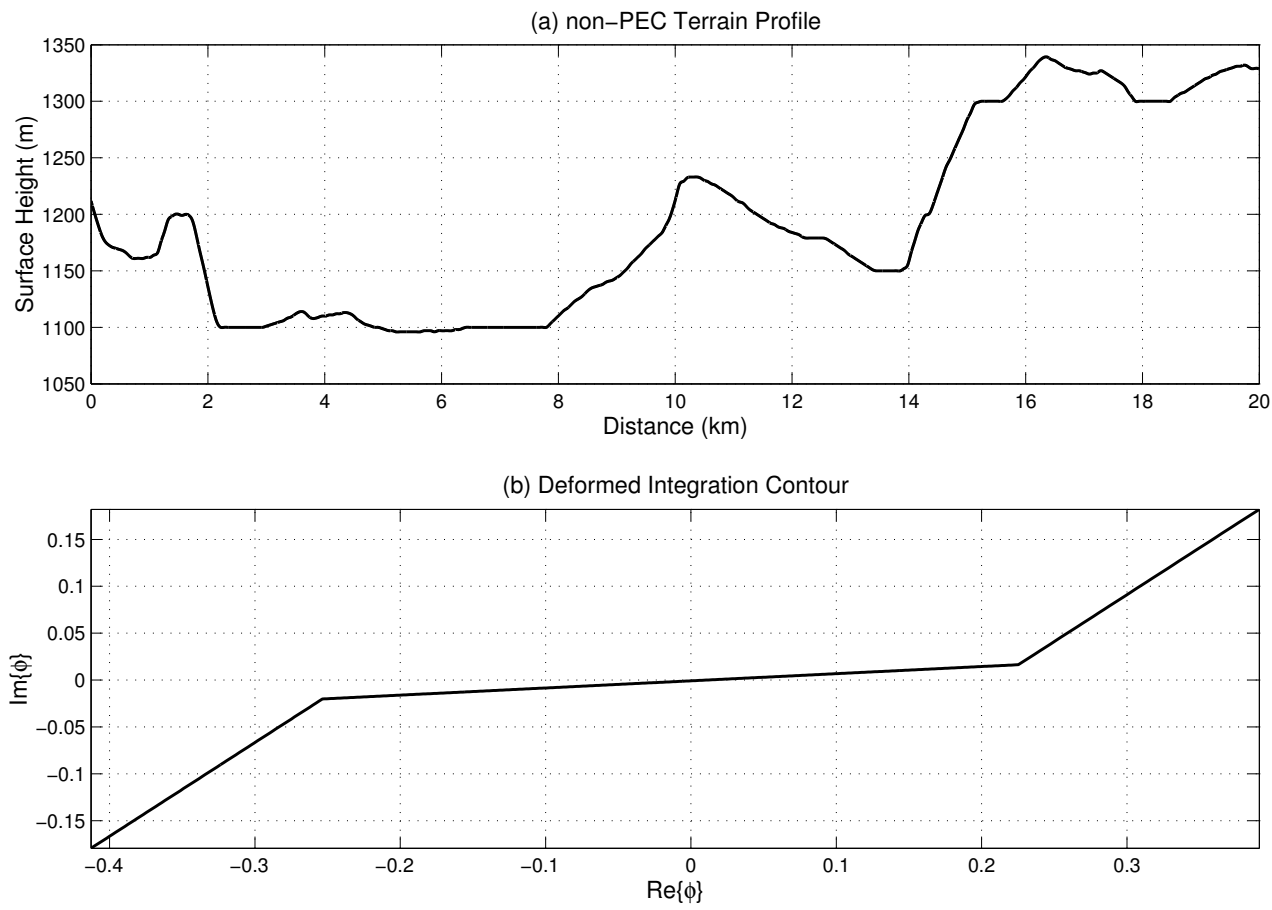

Figure 4.34: Deformed path of uphill profile at $890 \mathrm{MHz}$. 


\begin{tabular}{|c|c|c|}
\hline & Downhill & Uphill \\
\hline Frequency $(\mathrm{MHz})$ & 890 & 890 \\
\hline$\lambda(\mathrm{m})$ & 0.337 & 0.337 \\
\hline width $(\lambda)$ & 59333.3 & 59333.3 \\
\hline$N$ & 593333 & 593333 \\
\hline$\phi_{\text {med }}(\mathrm{rad})$ & -0.003 & 0.011 \\
\hline$\phi_{s, \text { min }}(\mathrm{rad})$ & -0.336 & -0.233 \\
\hline$\phi_{s, \text { max }}(\mathrm{rad})$ & 0.169 & 0.209 \\
\hline$L_{s}(\lambda)$ & 14.833 & 14.833 \\
\hline Num. of integr. points & 346 & 439 \\
\hline$\delta(\mathrm{rad})$ & 0.115 & 0.076 \\
\hline
\end{tabular}

Table 4.5: Study Parameters for $890 \mathrm{MHz}$

The FBSA results are obtained considering the horizontal and vertical cuts of the spherical pattern of an isotropic antenna located at the left-most end of the surfaces with a transmitted power of $P_{t}=50$ Watts, for TM and TE polarization cases, respectively, according to the parameters in Table 4.5.

The field strength predictions are evaluated using Free Space Propagation model, ITU-R recommendations; ITUR370, ITUR529, ITUR1546, and FCC Curves. Figures 4.35-4.39 show these comparisons over the downhill profile, while Figures 4.40-4.44 illustrate examinations over the uphill one; for the TM and TE polarization in the $f=890 \mathrm{MHz}$ case.

Table 4.6 shows the absolute error values for different propagation models and polarizations over uphill and downhill profiles for $890 \mathrm{MHz}$. It is observed that for an isotropic source having $890 \mathrm{MHz}$ operating frequency, using FCC curves, free space propagation or ITUR-529 model is preferable than the others over downhill profiles in TE and TM polarization cases. However, FCC curves with multiple diffraction is the most preferable model among others, and the worst model seems to be the ITUR-1546, again. 
(a) non-PEC Terrain Profile

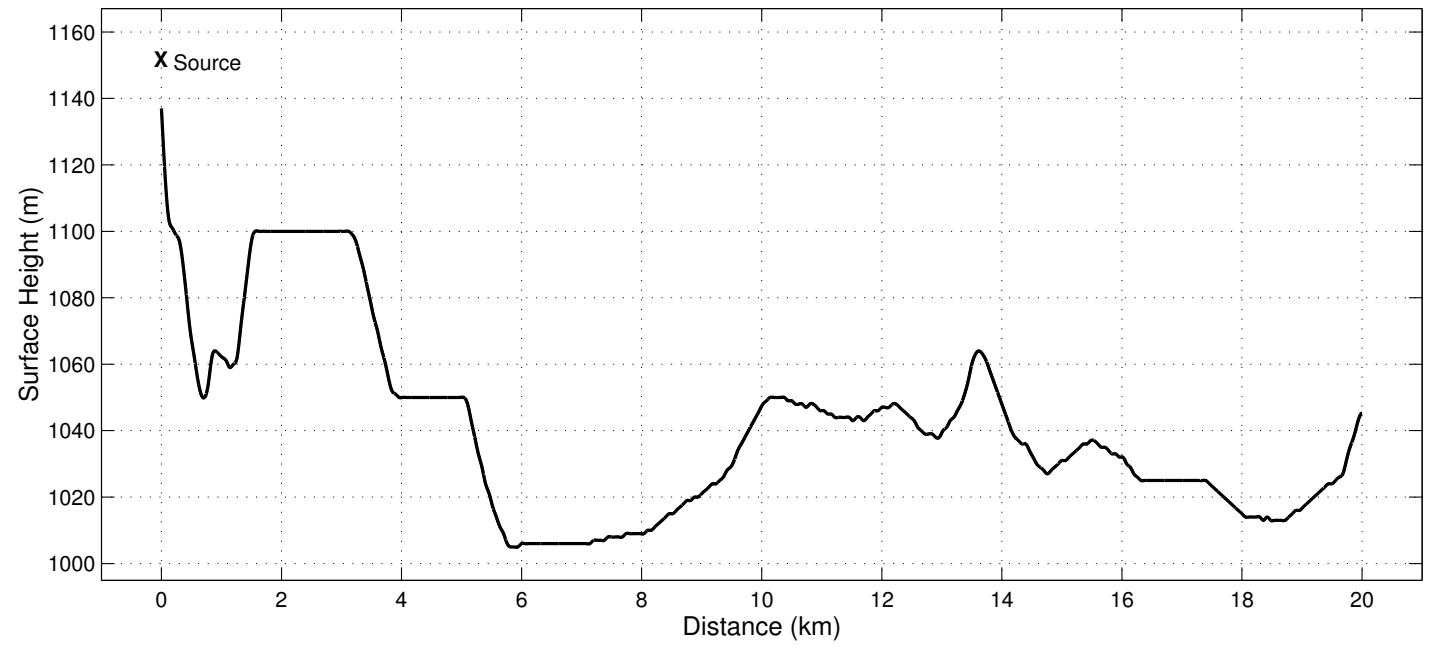

(b) TM Polarized Isotropic Radiator

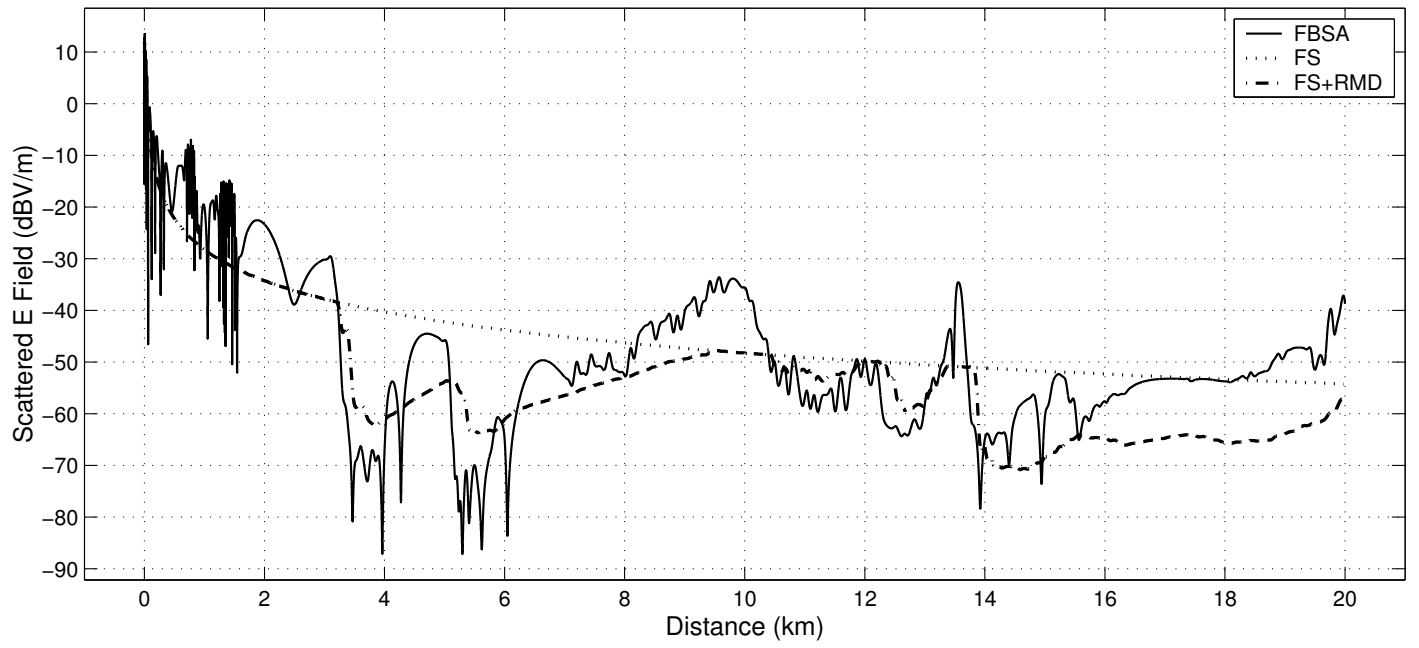

(c) TE Polarized Isotropic Radiator

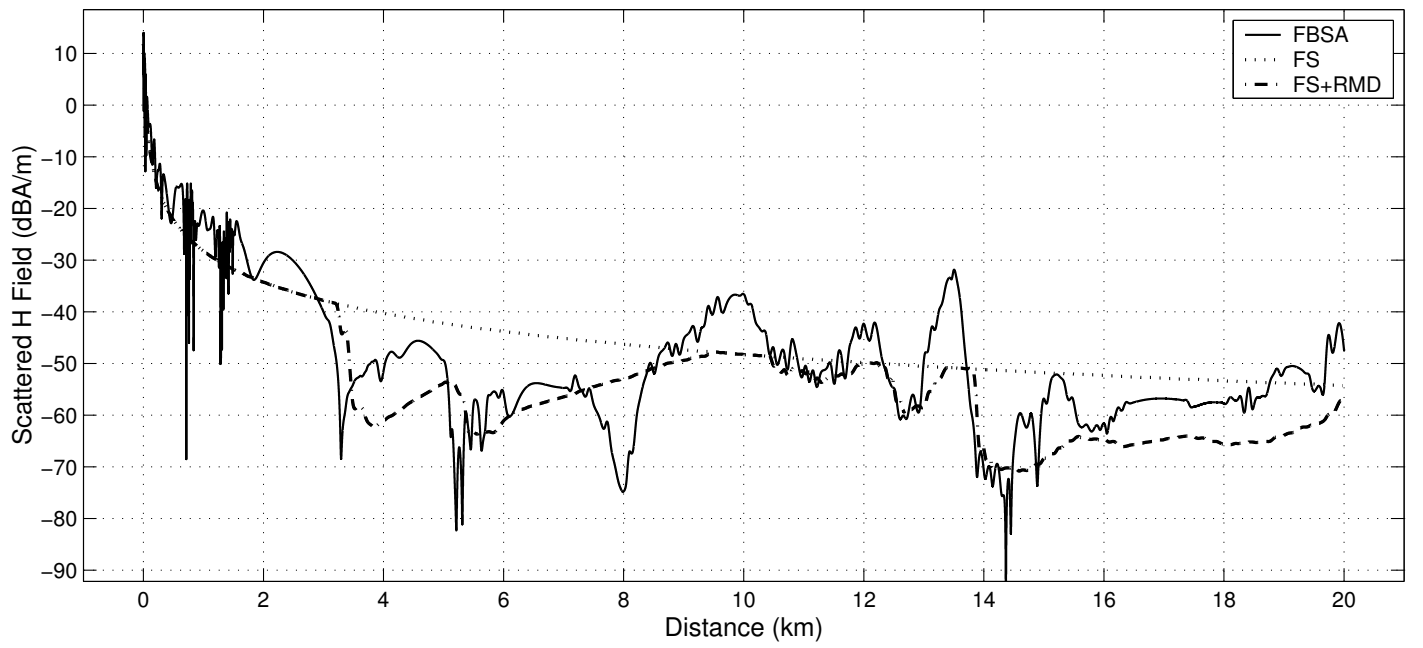

Figure 4.35: FreeSpace vs. IE over downhill terrain for $890 \mathrm{MHz}$. 
(a) non-PEC Terrain Profile

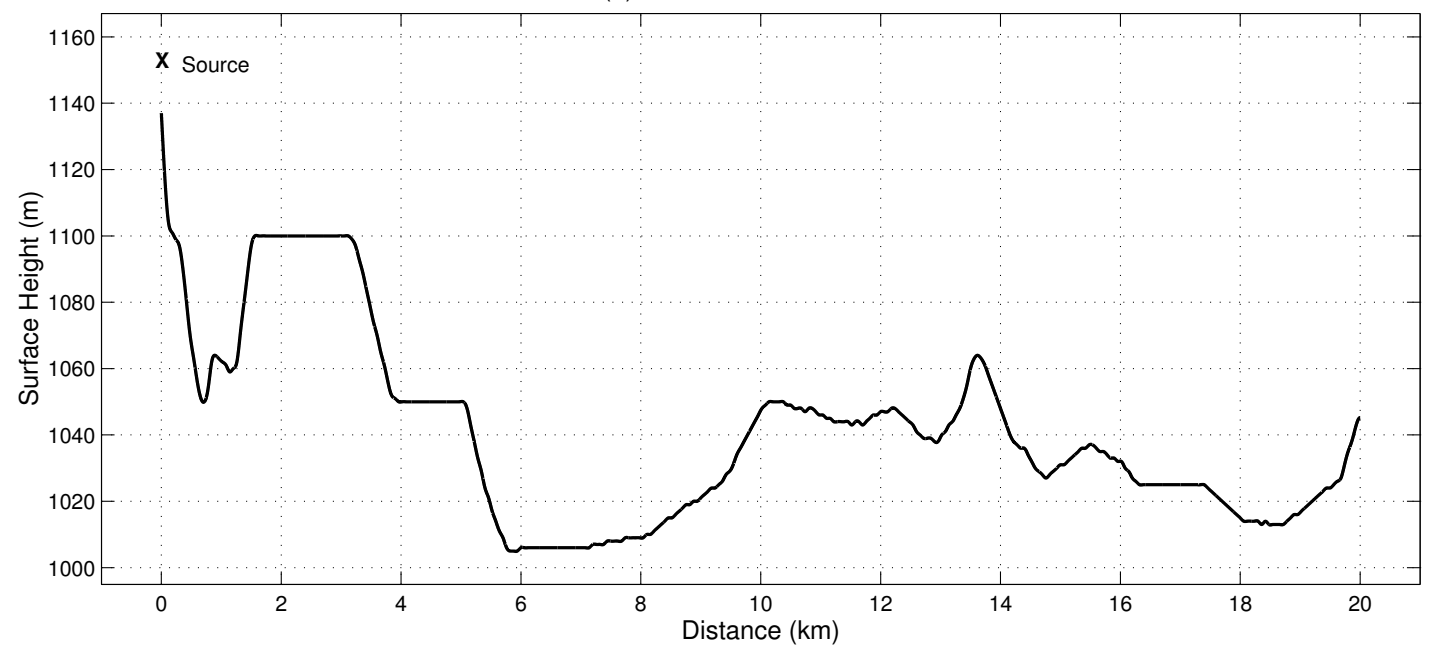

(b) TM Polarized Isotropic Radiator

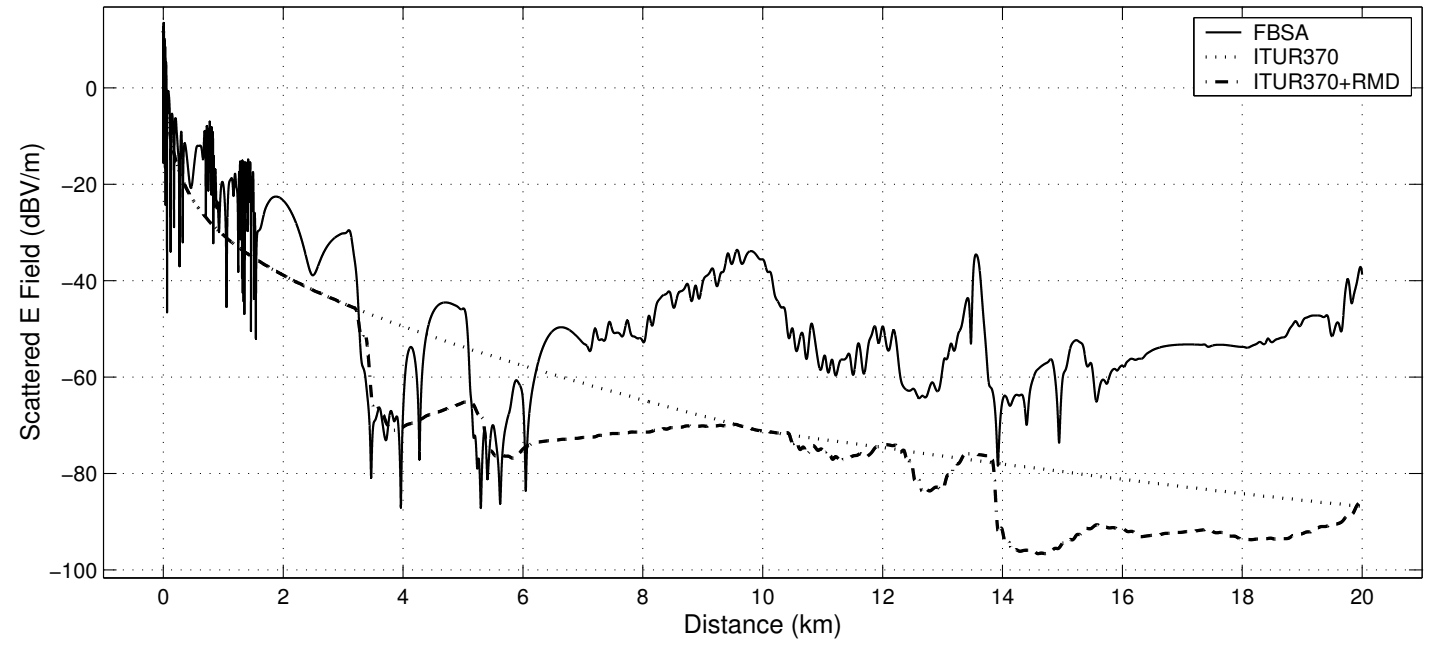

(c) TE Polarized Isotropic Radiator

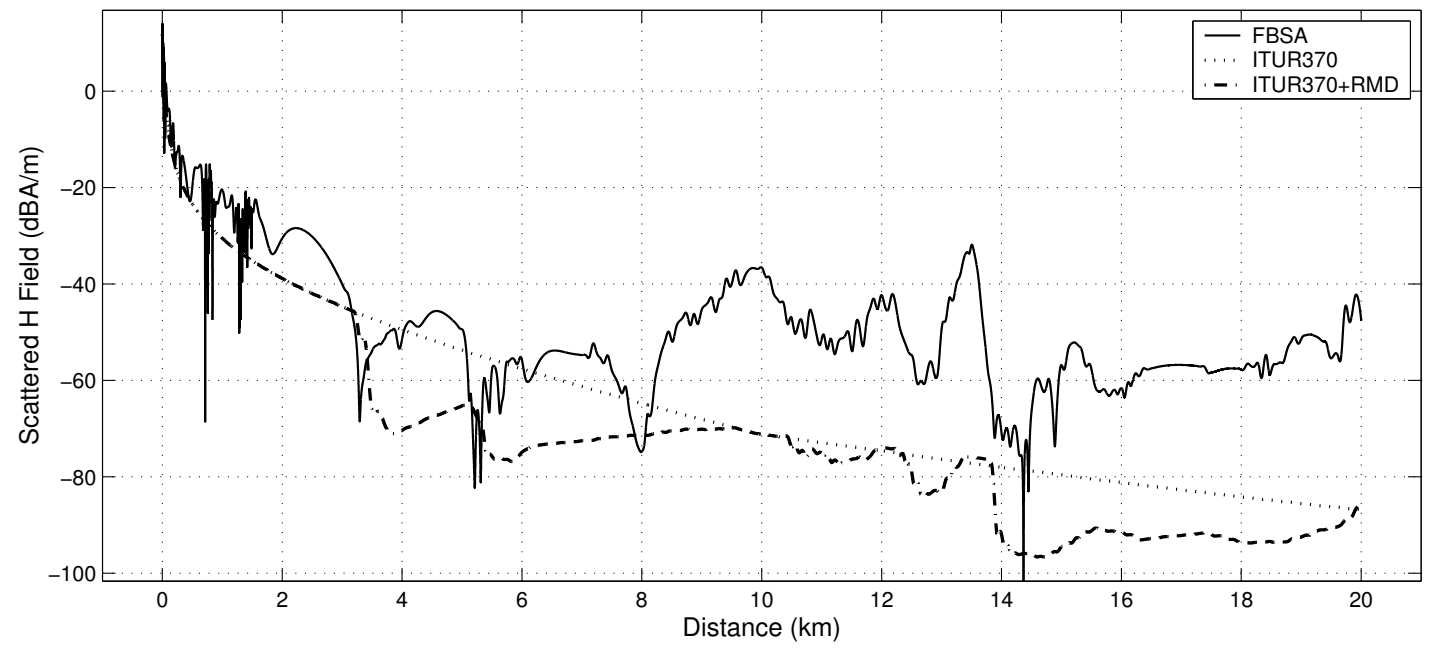

Figure 4.36: ITUR370 vs. IE over downhill terrain for $890 \mathrm{MHz}$. 
(a) non-PEC Terrain Profile

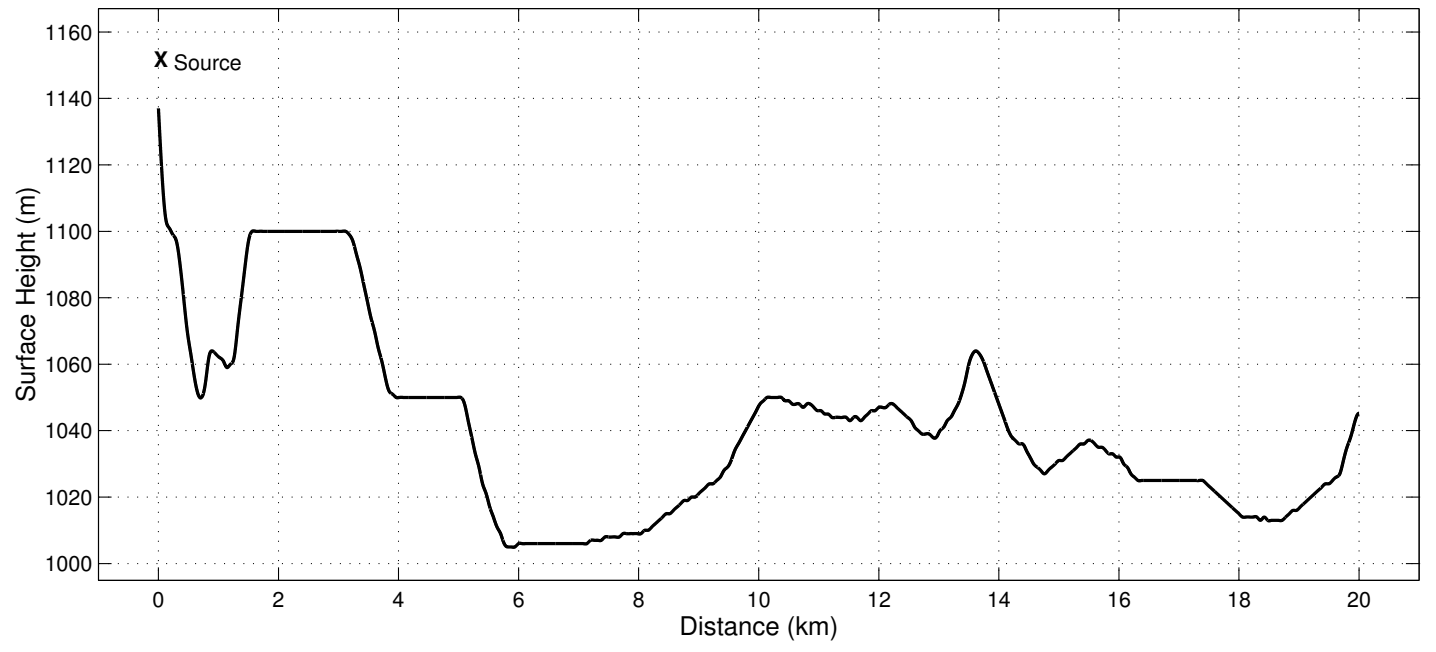

(b) TM Polarized Isotropic Radiator

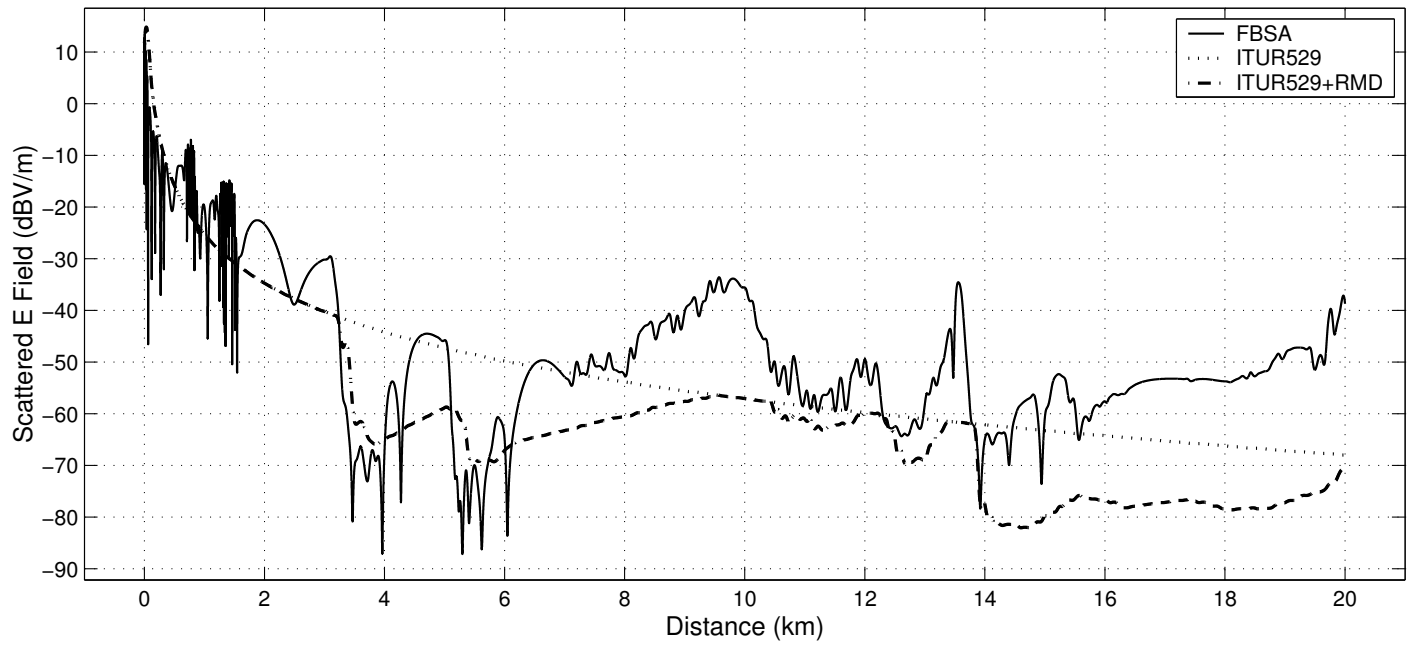

(c) TE Polarized Isotropic Radiator

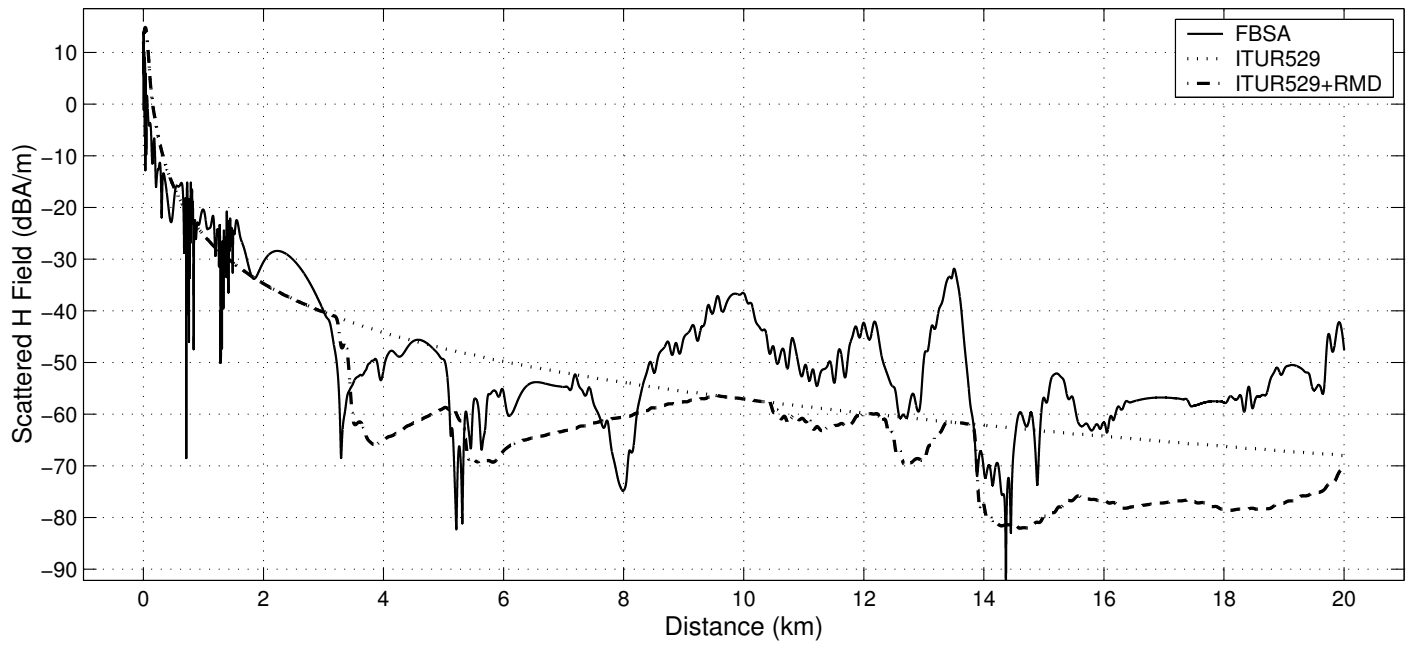

Figure 4.37: ITUR529 vs. IE over downhill terrain for $890 \mathrm{MHz}$. 
(a) non-PEC Terrain Profile

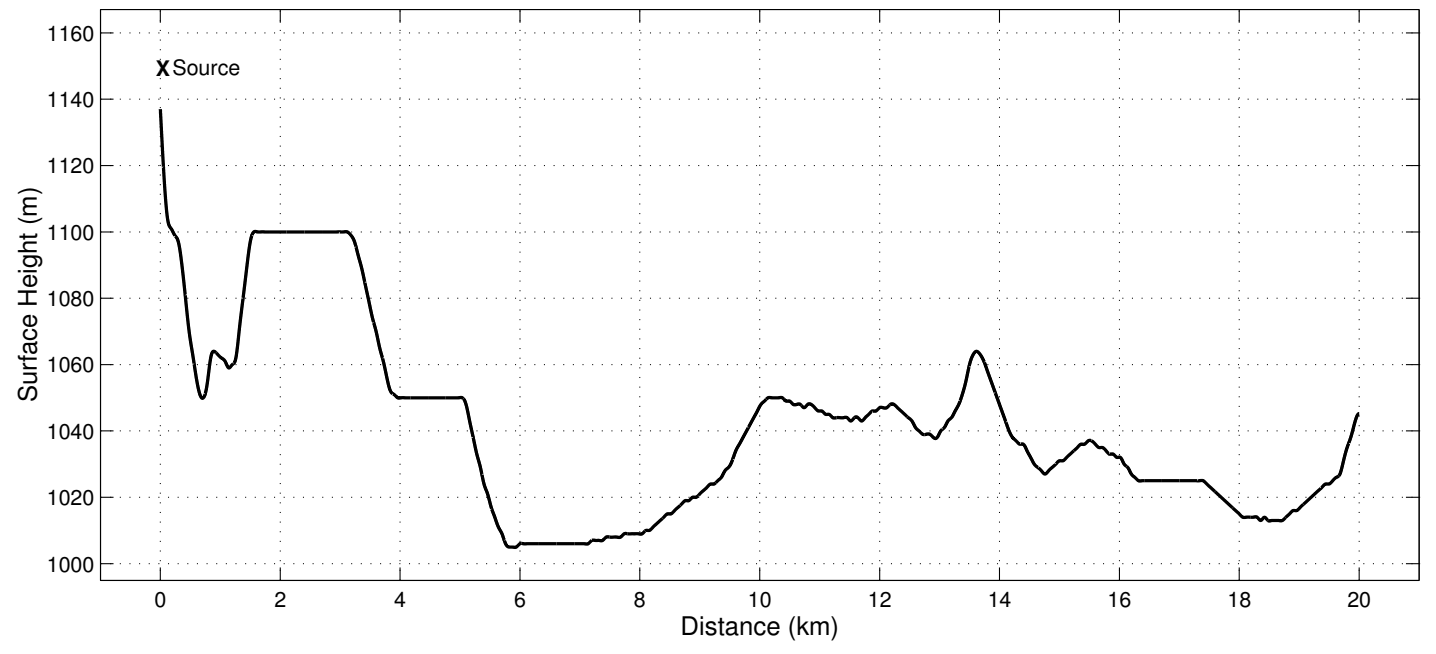

(b) TM Polarized Isotropic Radiator

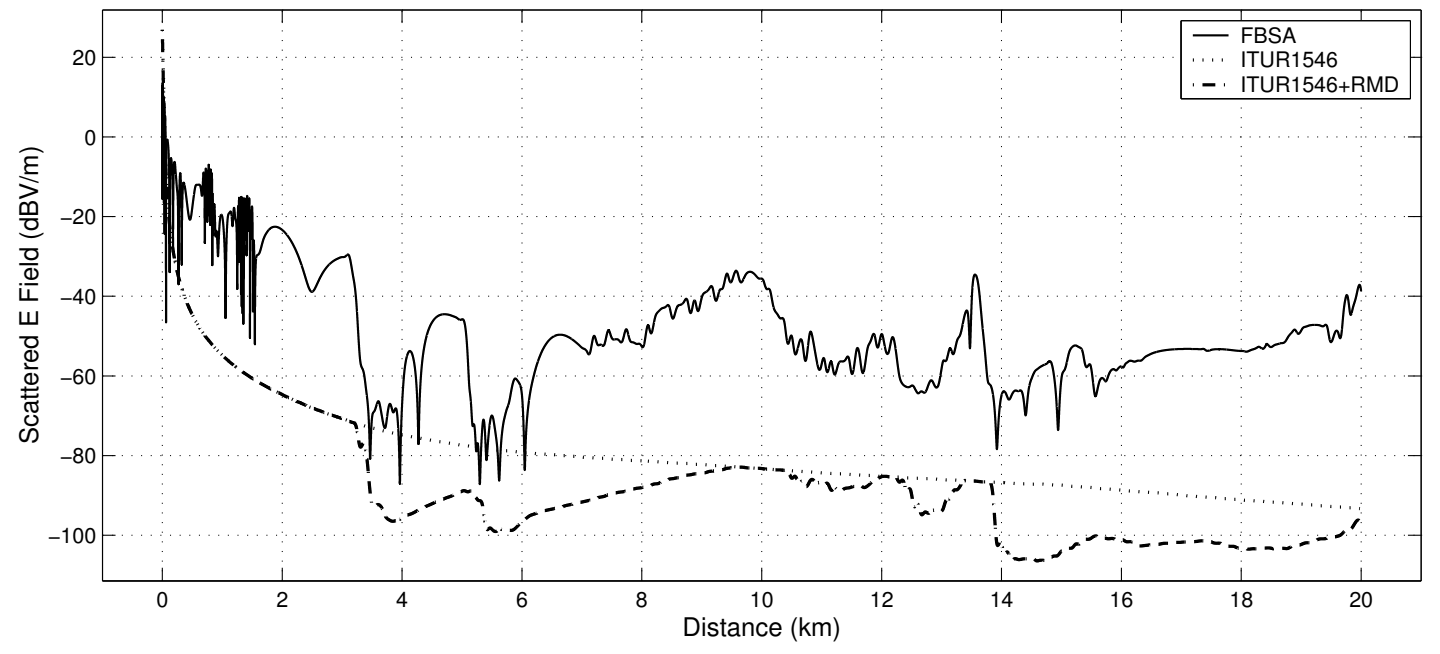

(c) TE Polarized Isotropic Radiator

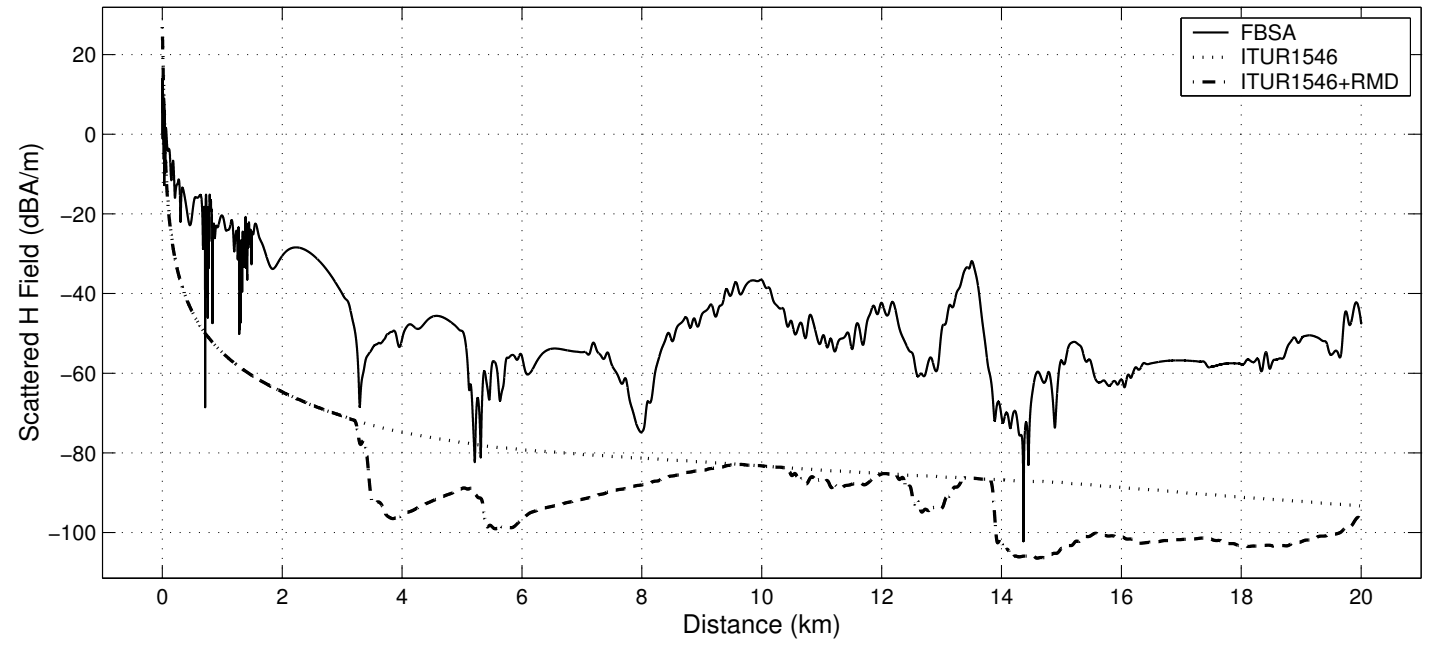

Figure 4.38: ITUR1546 vs. IE over downhill terrain for $890 \mathrm{MHz}$. 
(a) non-PEC Terrain Profile

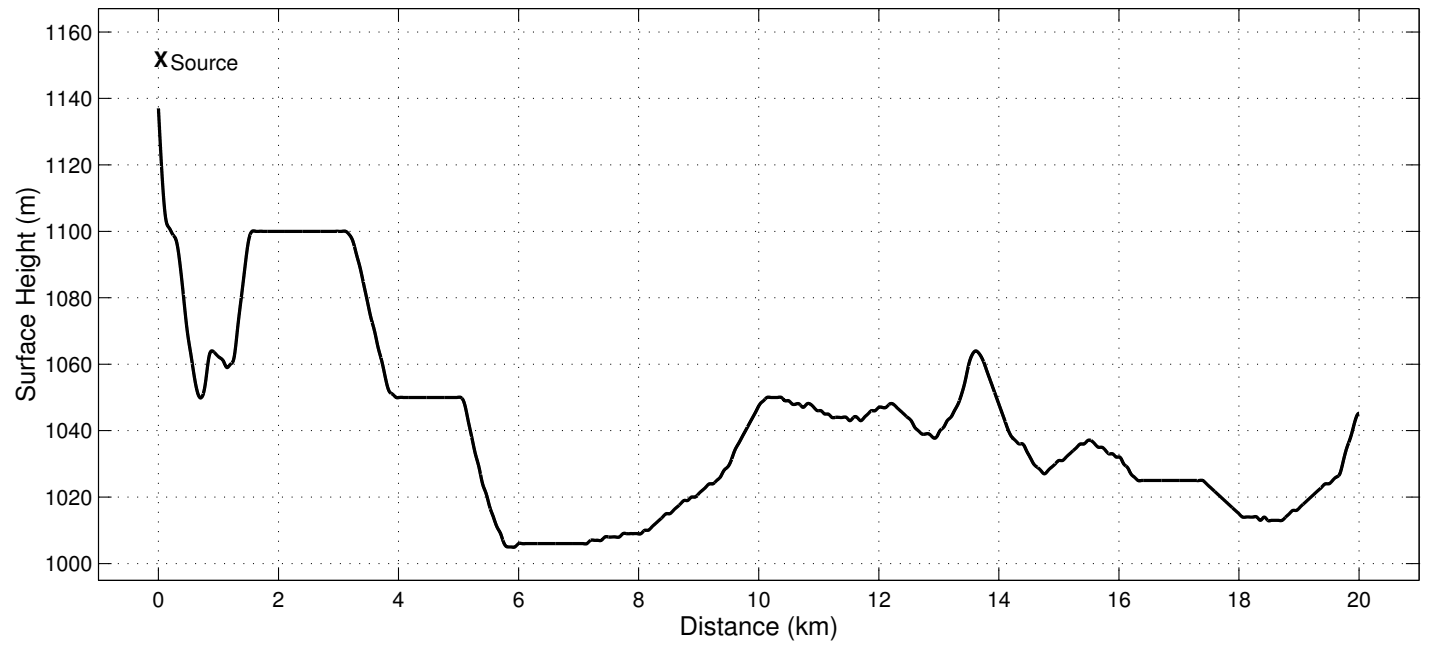

(b) TM Polarized Isotropic Radiator

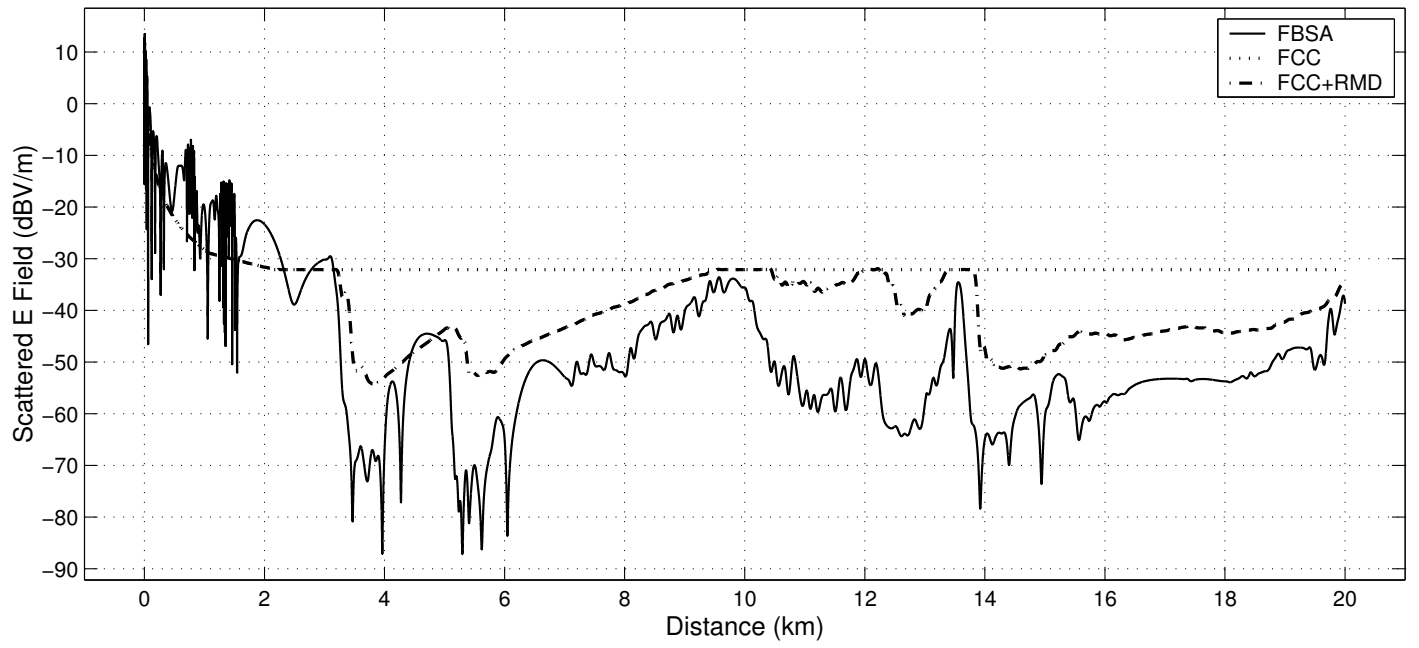

(c) TE Polarized Isotropic Radiator

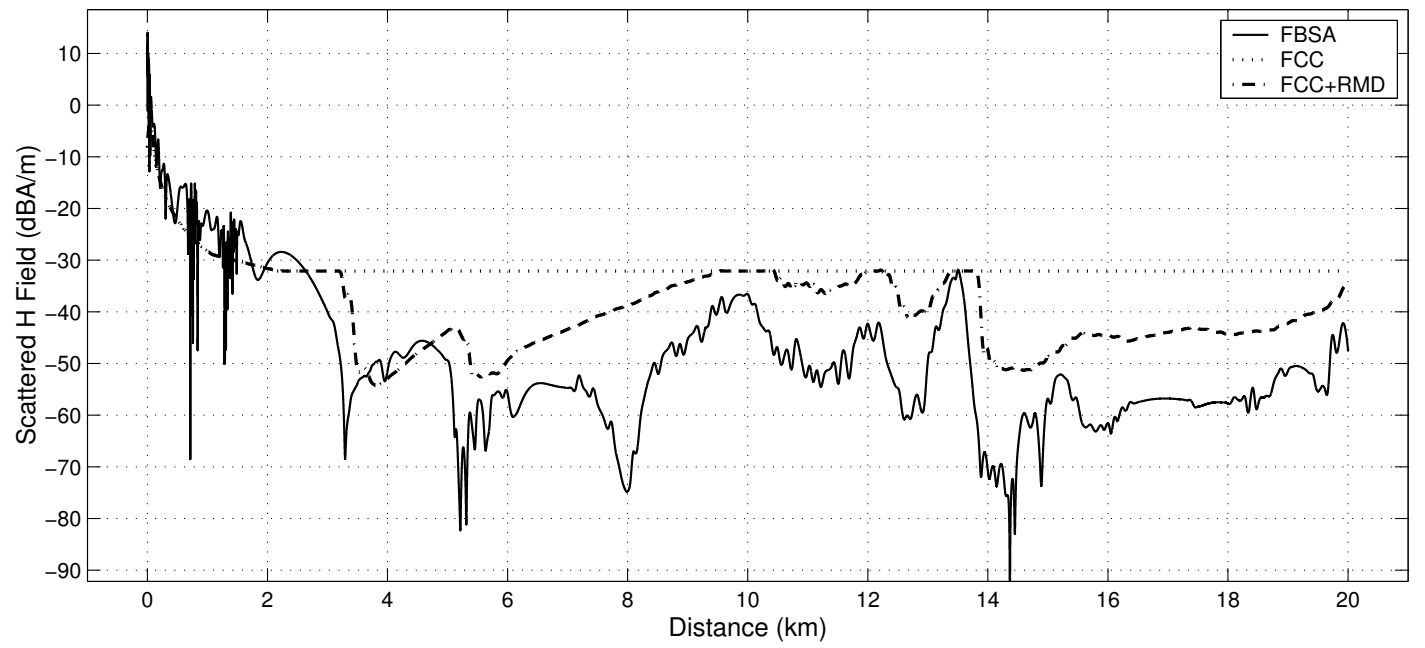

Figure 4.39: FCC vs. IE over downhill terrain for $890 \mathrm{MHz}$. 
(a) non-PEC Terrain Profile

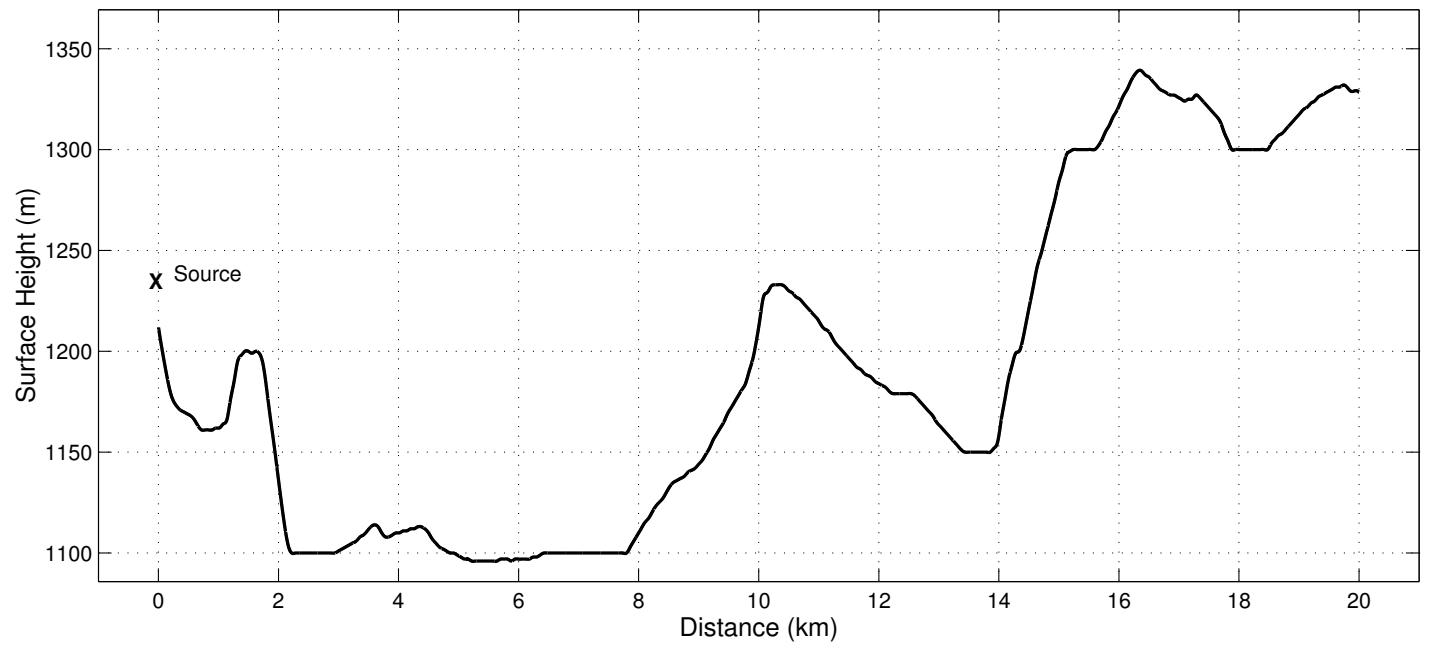

(b) TM Polarized Isotropic Radiator

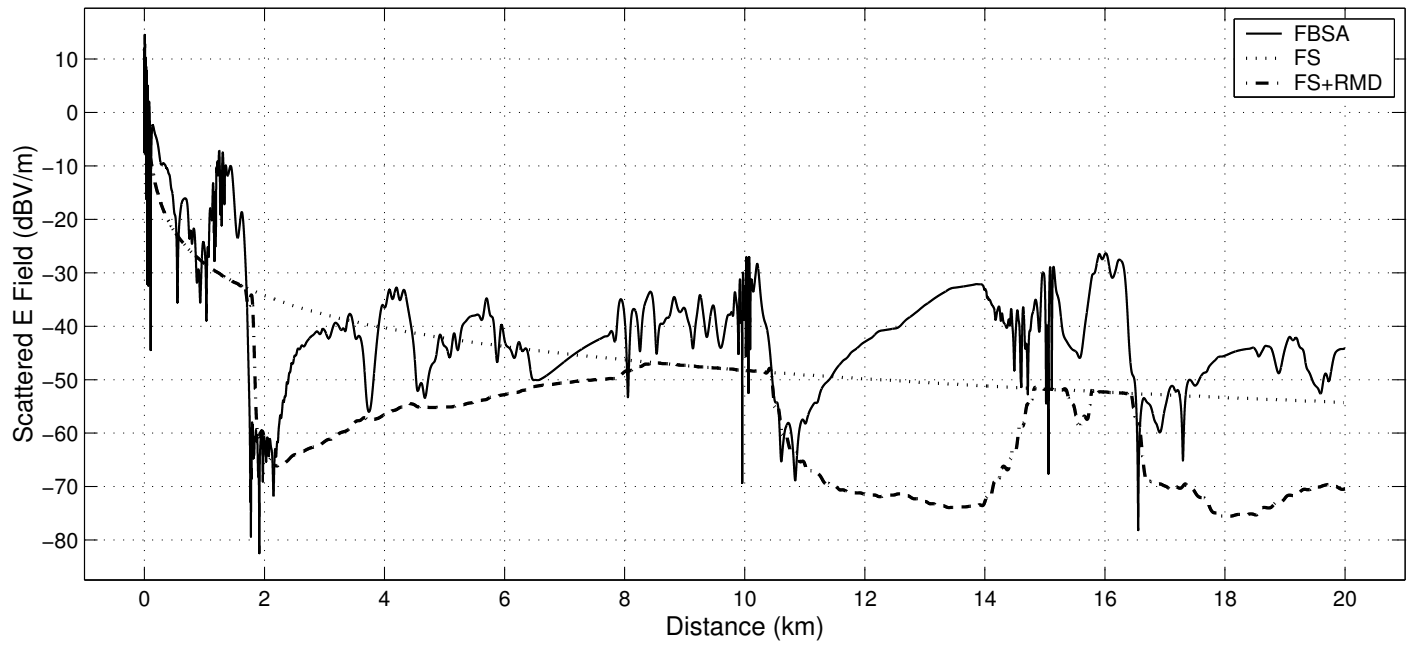

(c) TE Polarized Isotropic Radiator

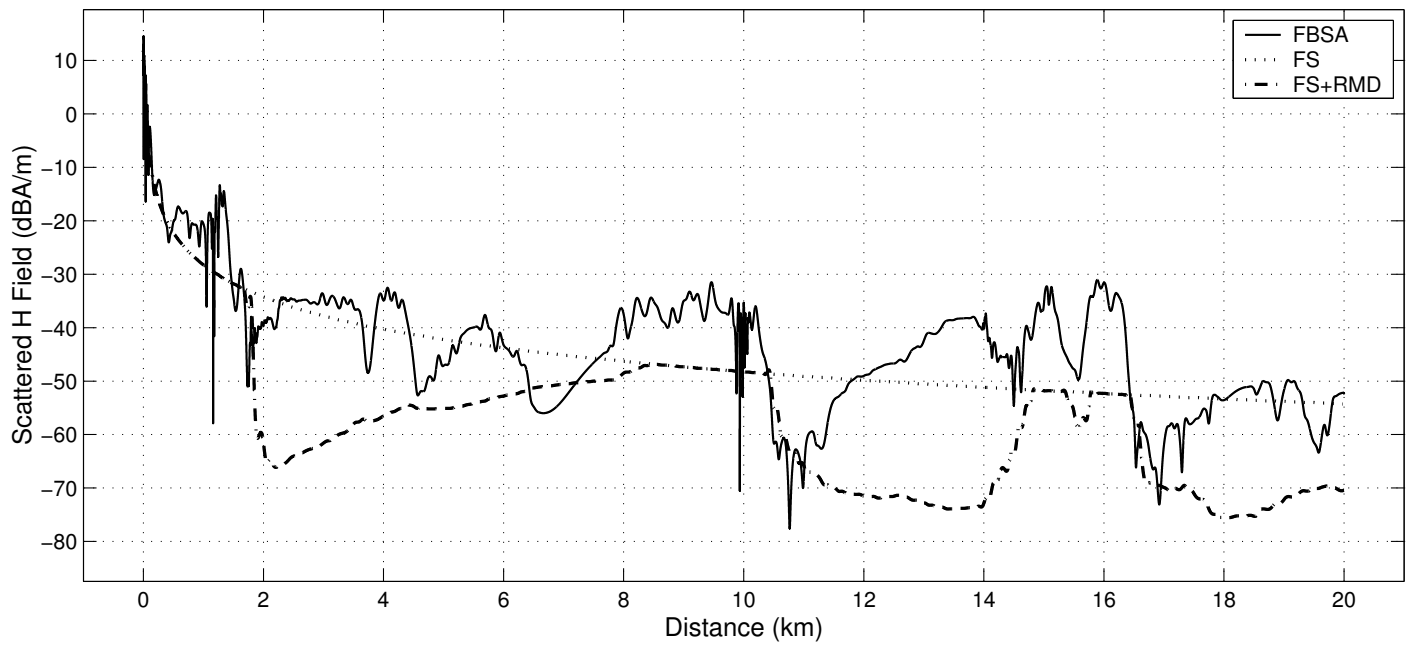

Figure 4.40: FreeSpace vs. IE over uphill terrain for $890 \mathrm{MHz}$. 
(a) non-PEC Terrain Profile

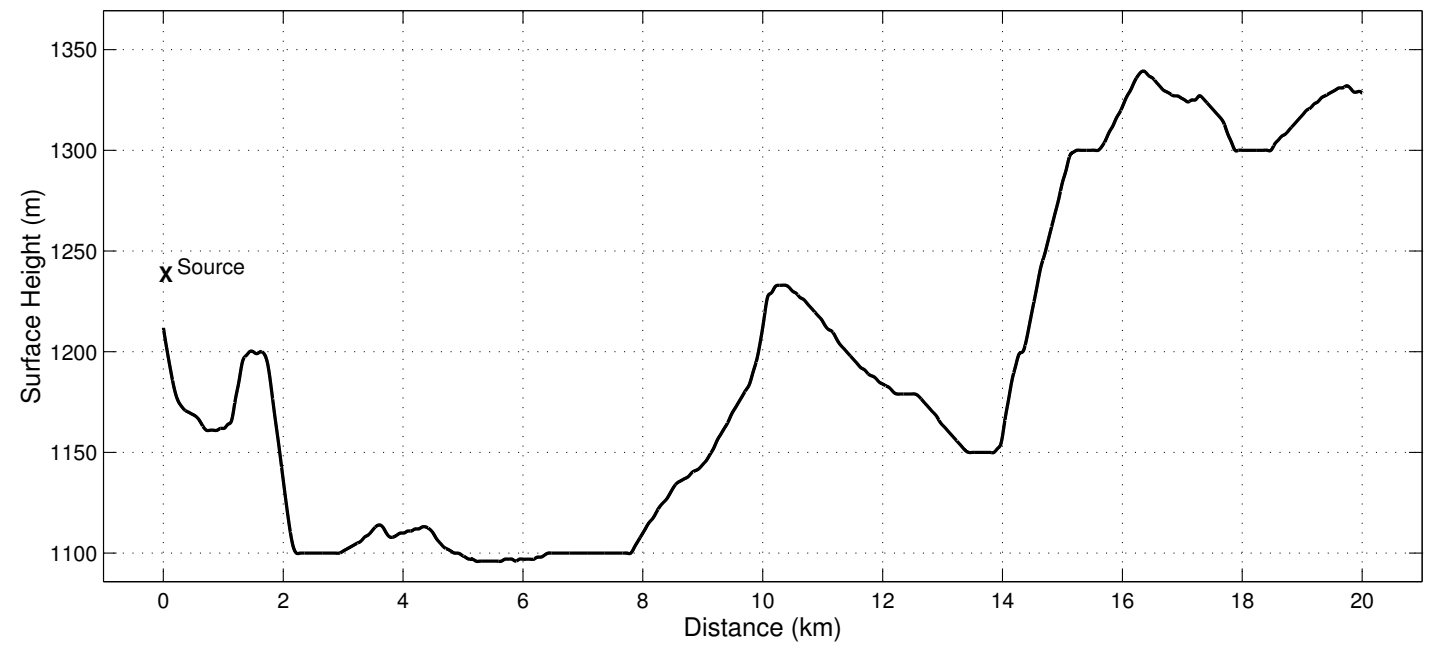

(b) TM Polarized Isotropic Radiator

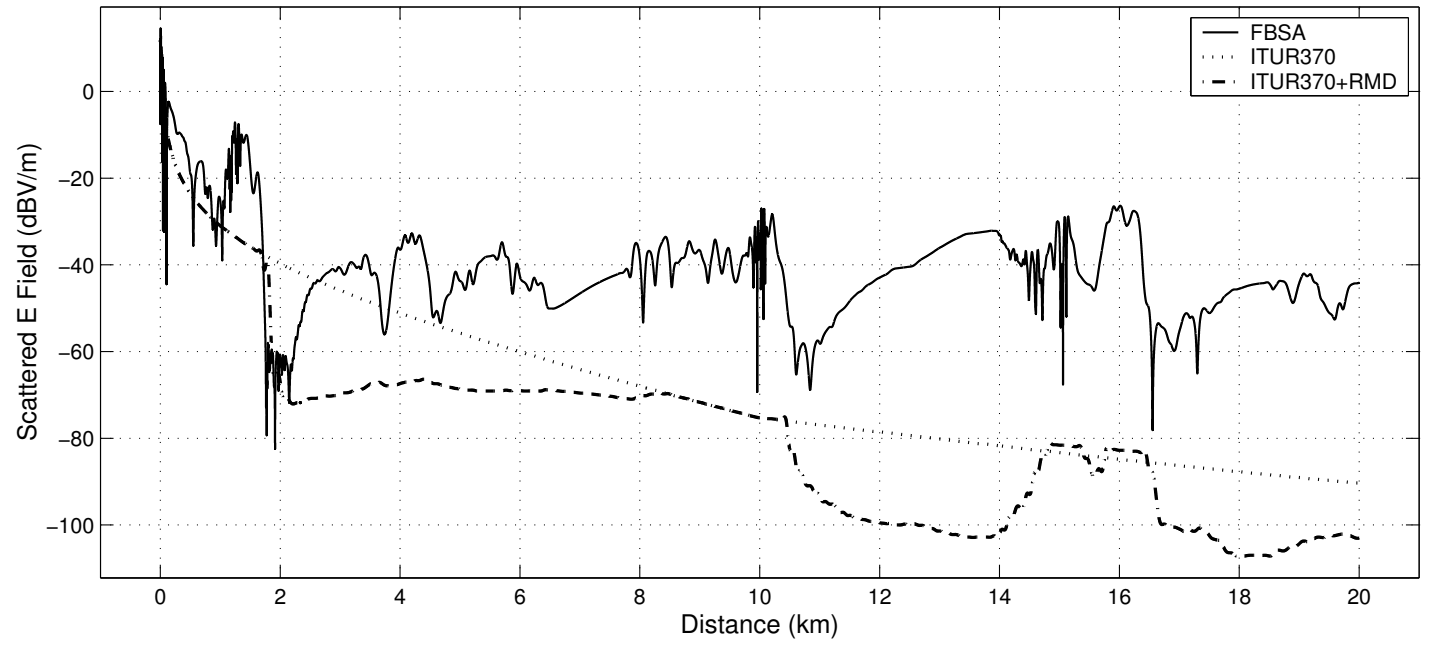

(c) TE Polarized Isotropic Radiator

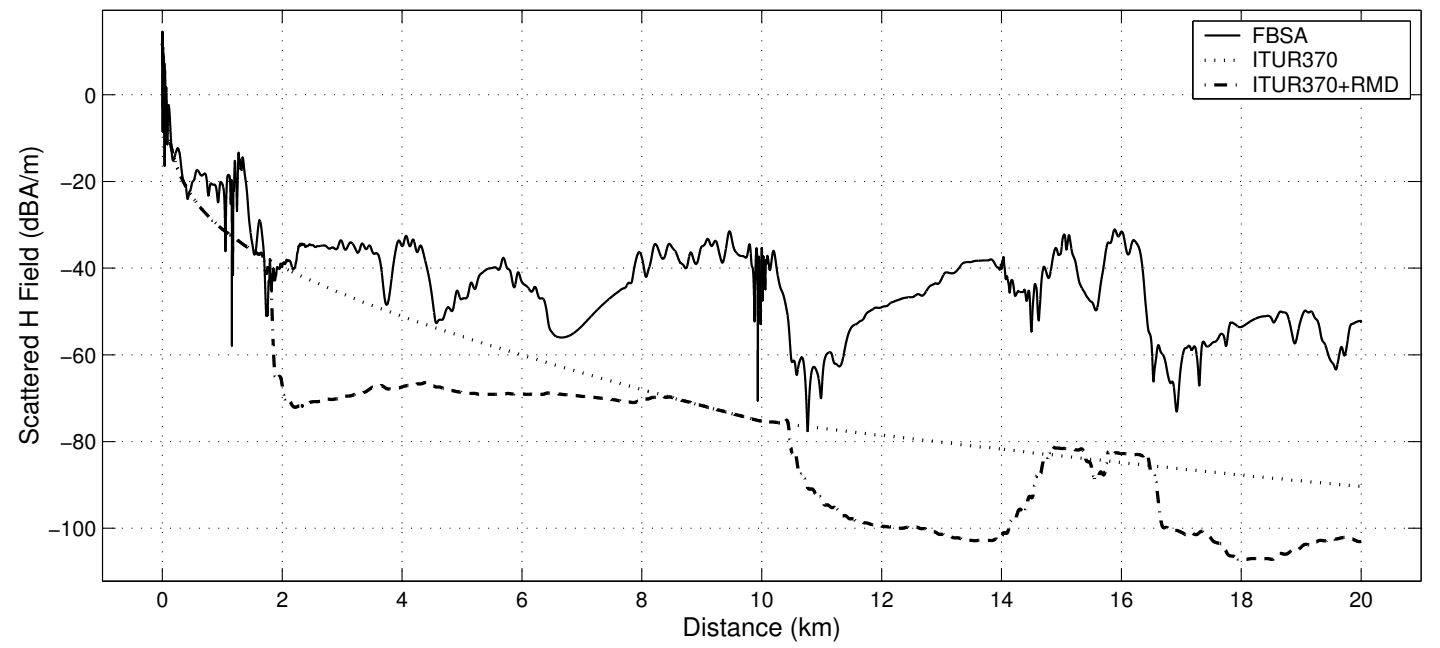

Figure 4.41: ITUR370 vs. IE over uphill terrain for $890 \mathrm{MHz}$. 
(a) non-PEC Terrain Profile

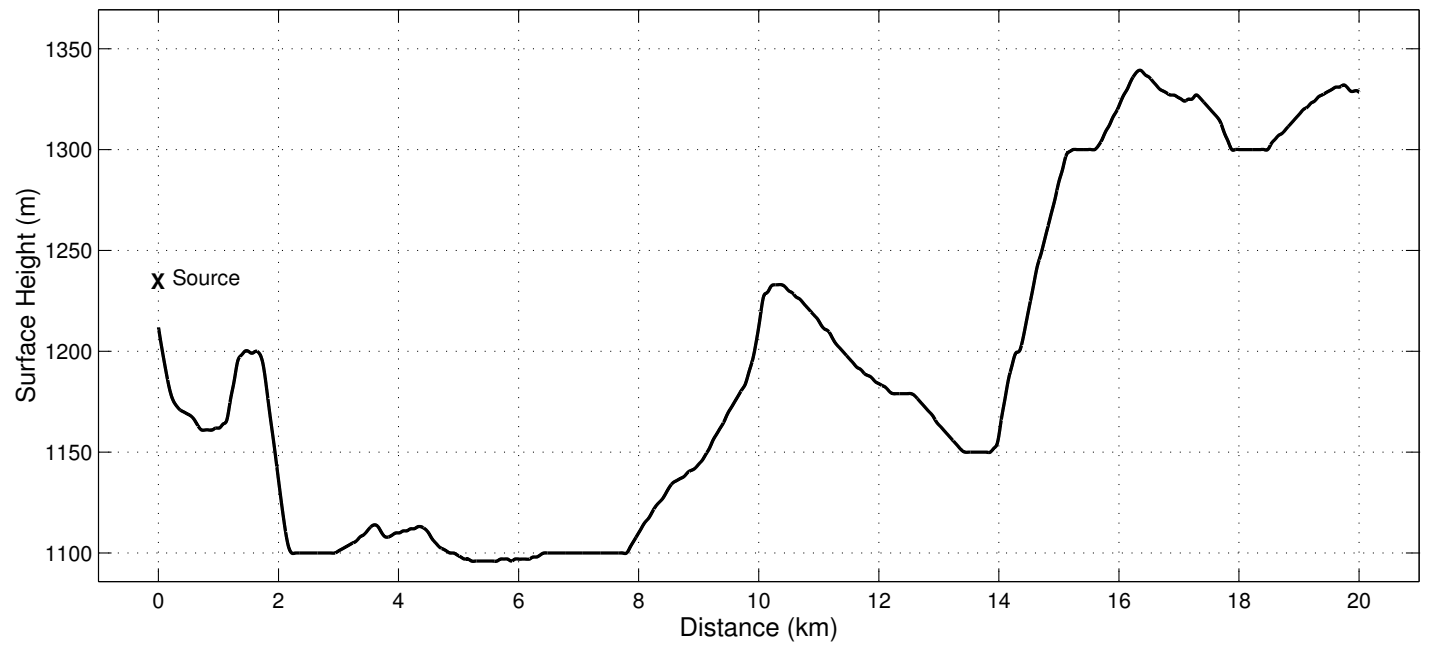

(b) TM Polarized Isotropic Radiator

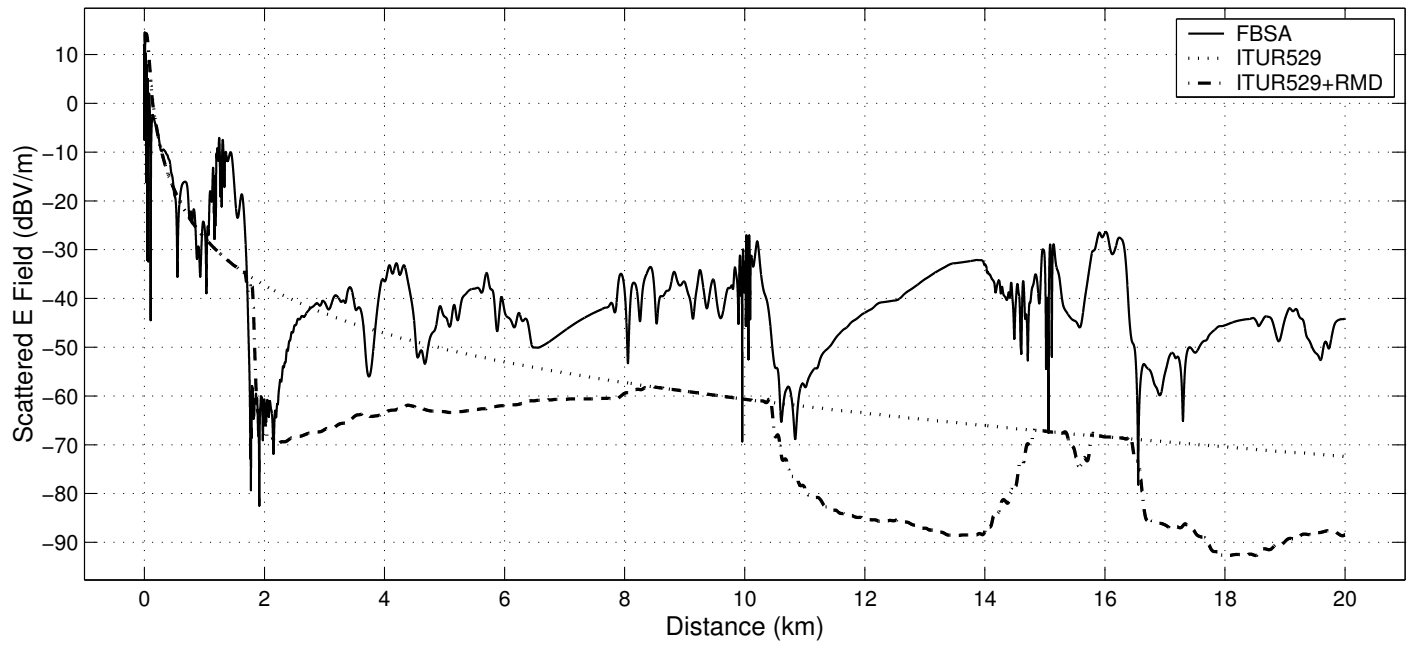

(c) TE Polarized Isotropic Radiator

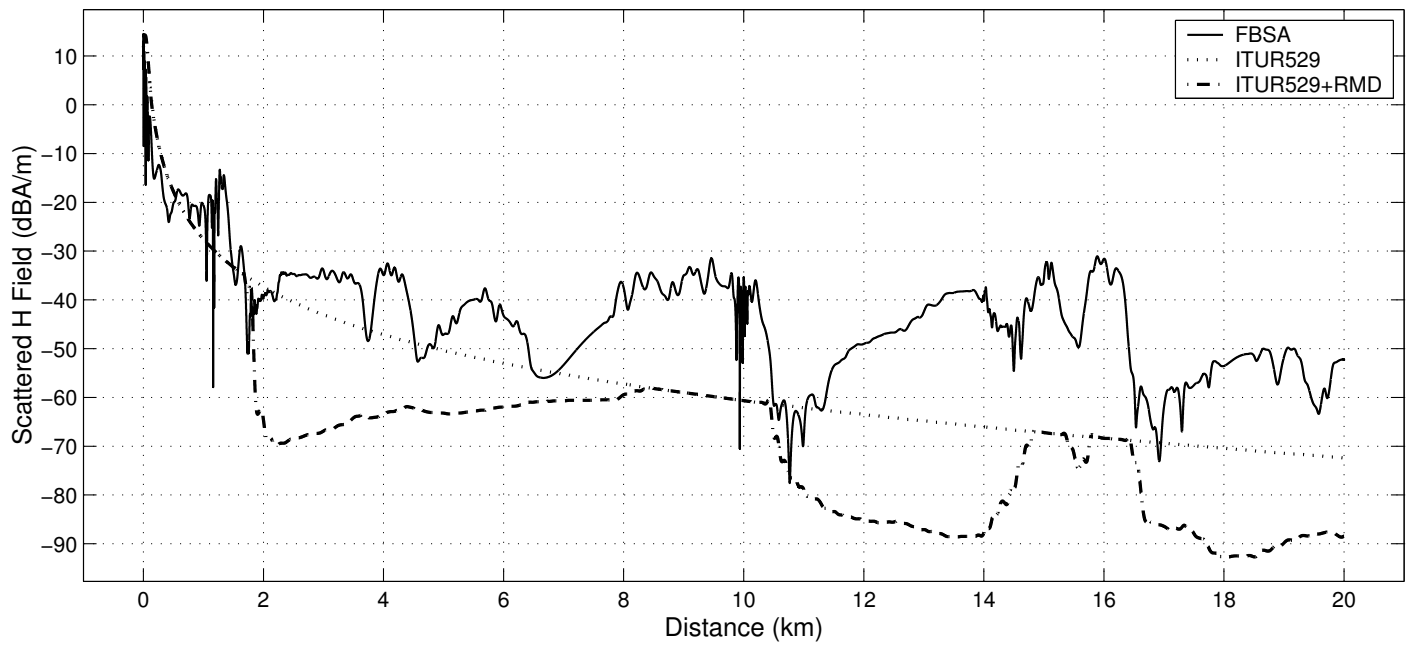

Figure 4.42: ITUR529 vs. IE over uphill terrain for $890 \mathrm{MHz}$. 
(a) non-PEC Terrain Profile

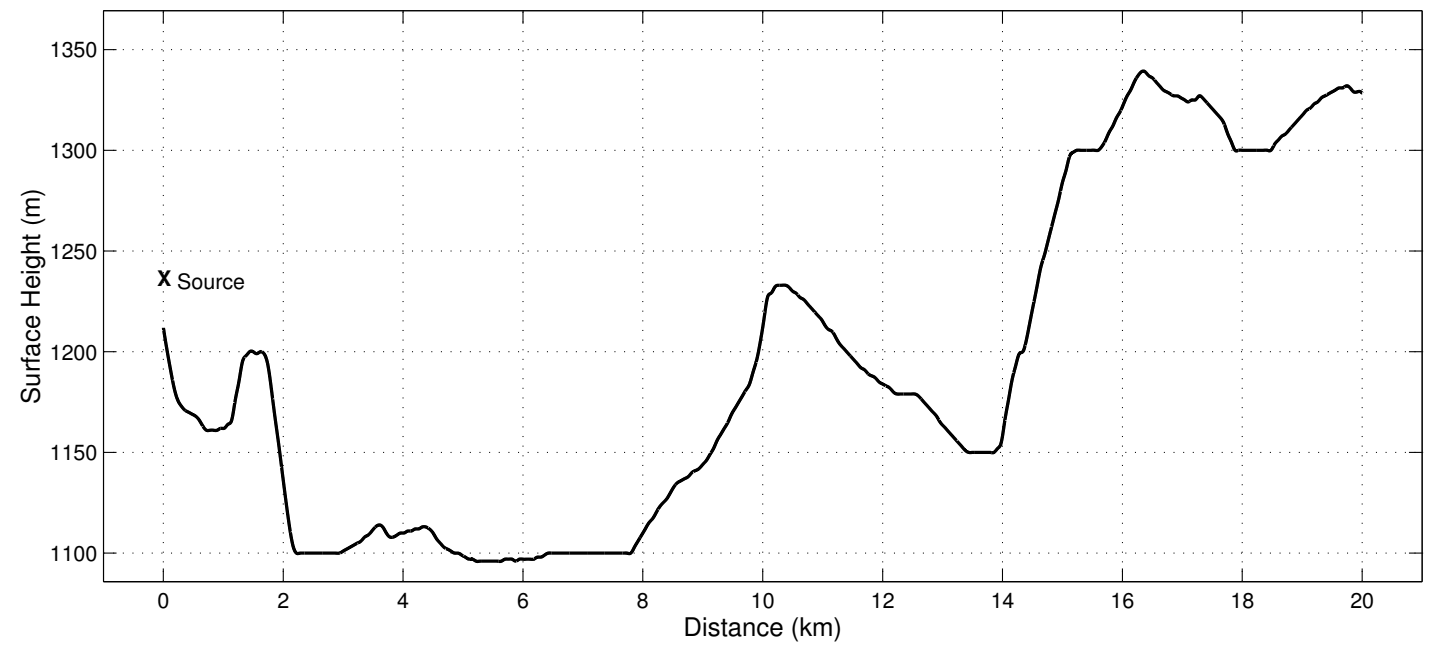

(b) TM Polarized Isotropic Radiator

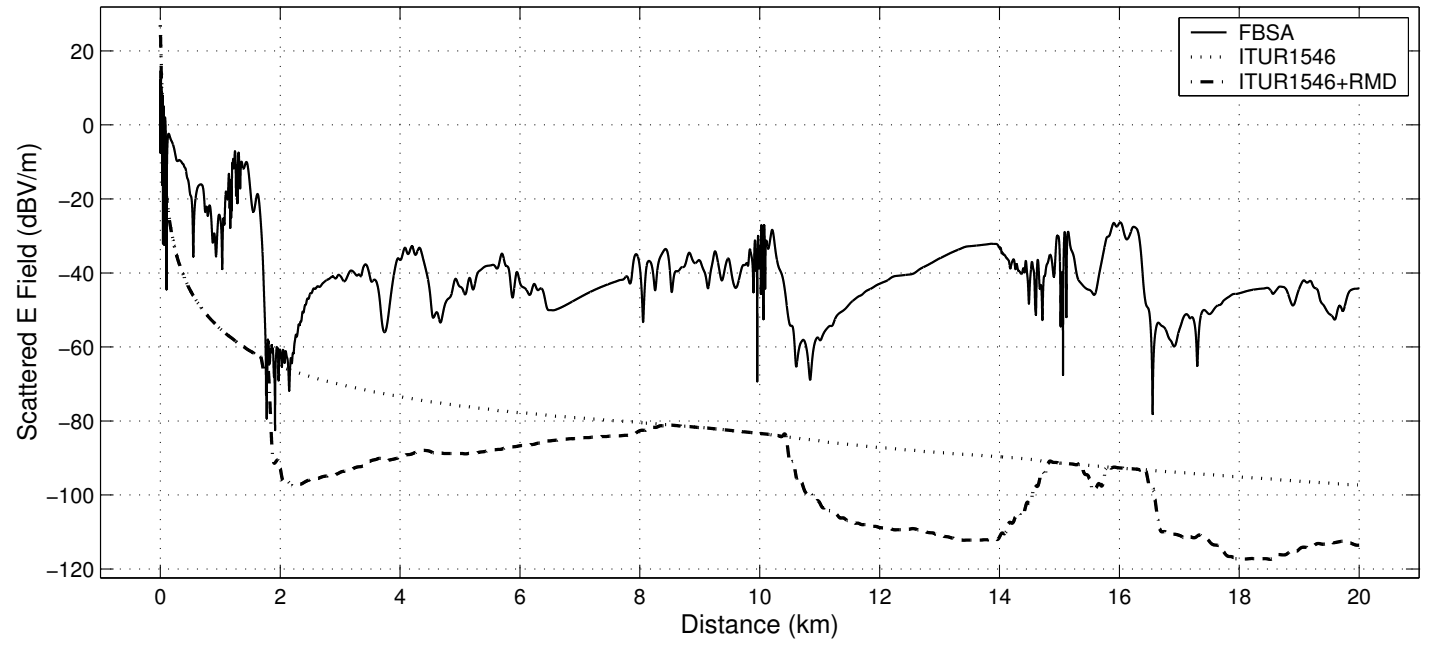

(c) TE Polarized Isotropic Radiator

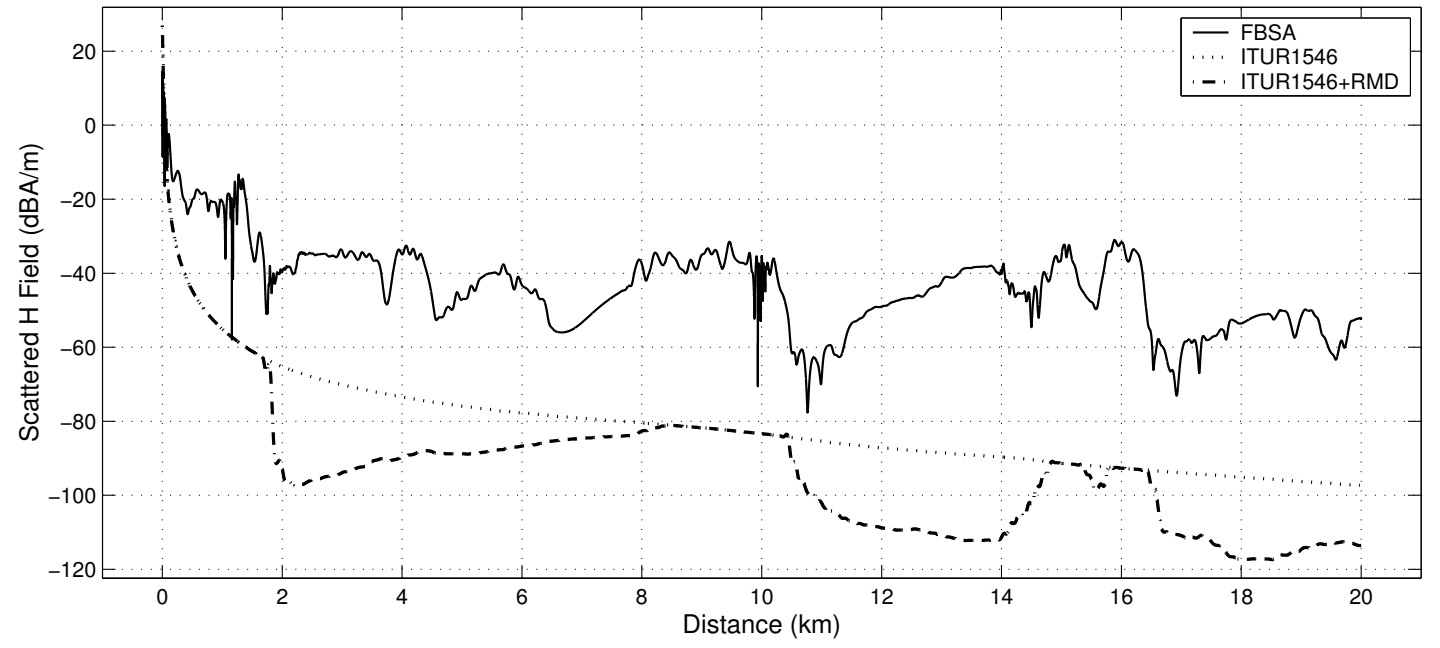

Figure 4.43: ITUR1546 vs. IE over uphill terrain for $890 \mathrm{MHz}$. 
(a) non-PEC Terrain Profile

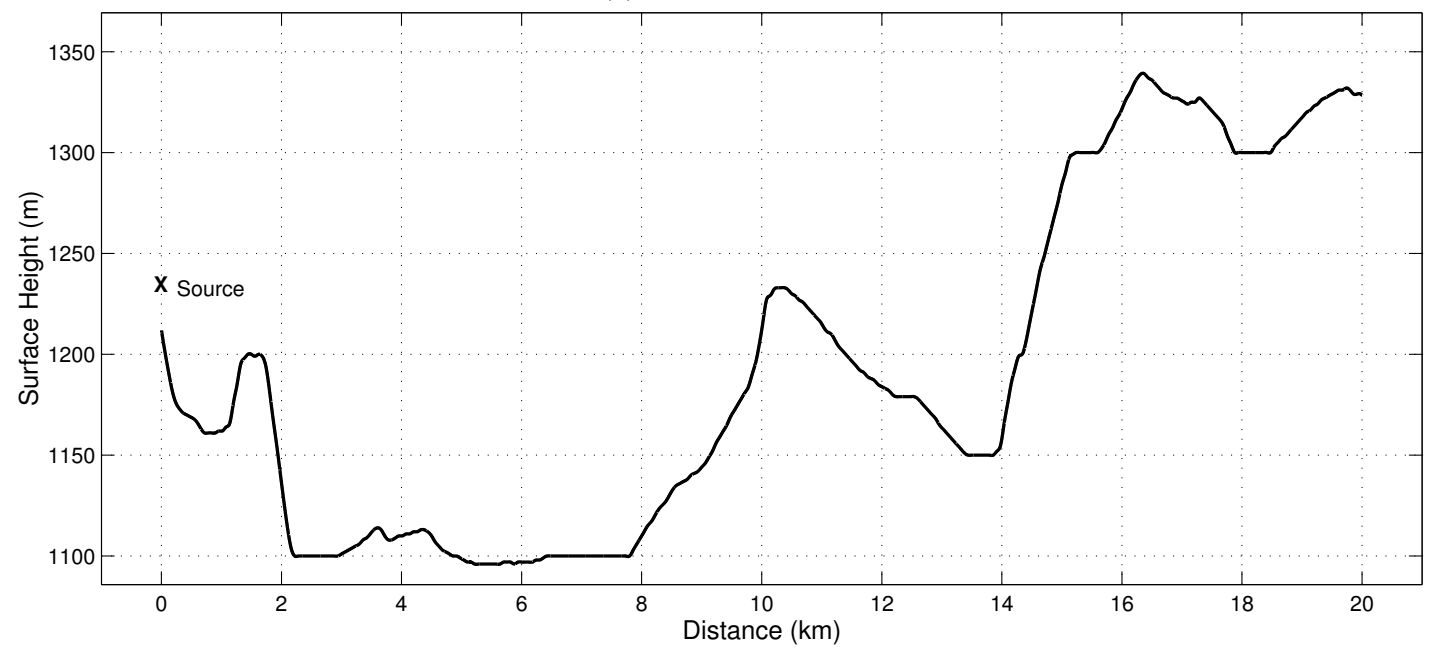

(b) TM Polarized Isotropic Radiator

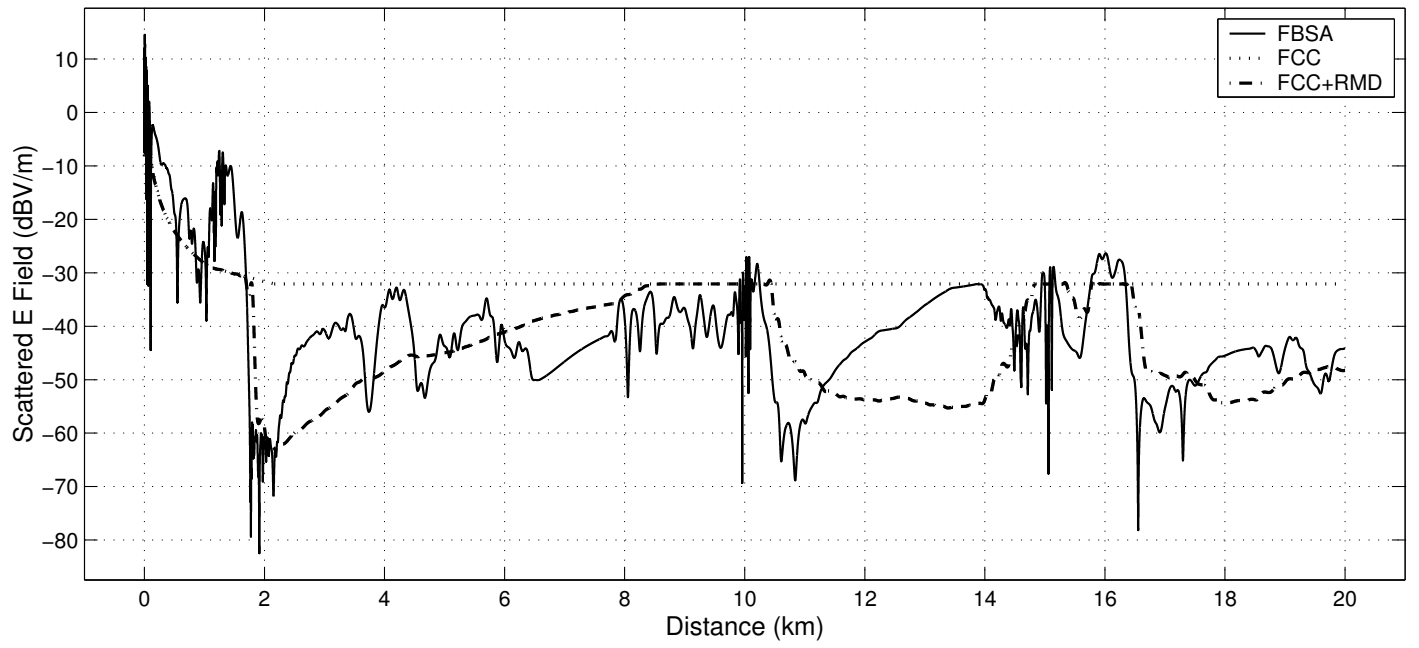

(c) TE Polarized Isotropic Radiator

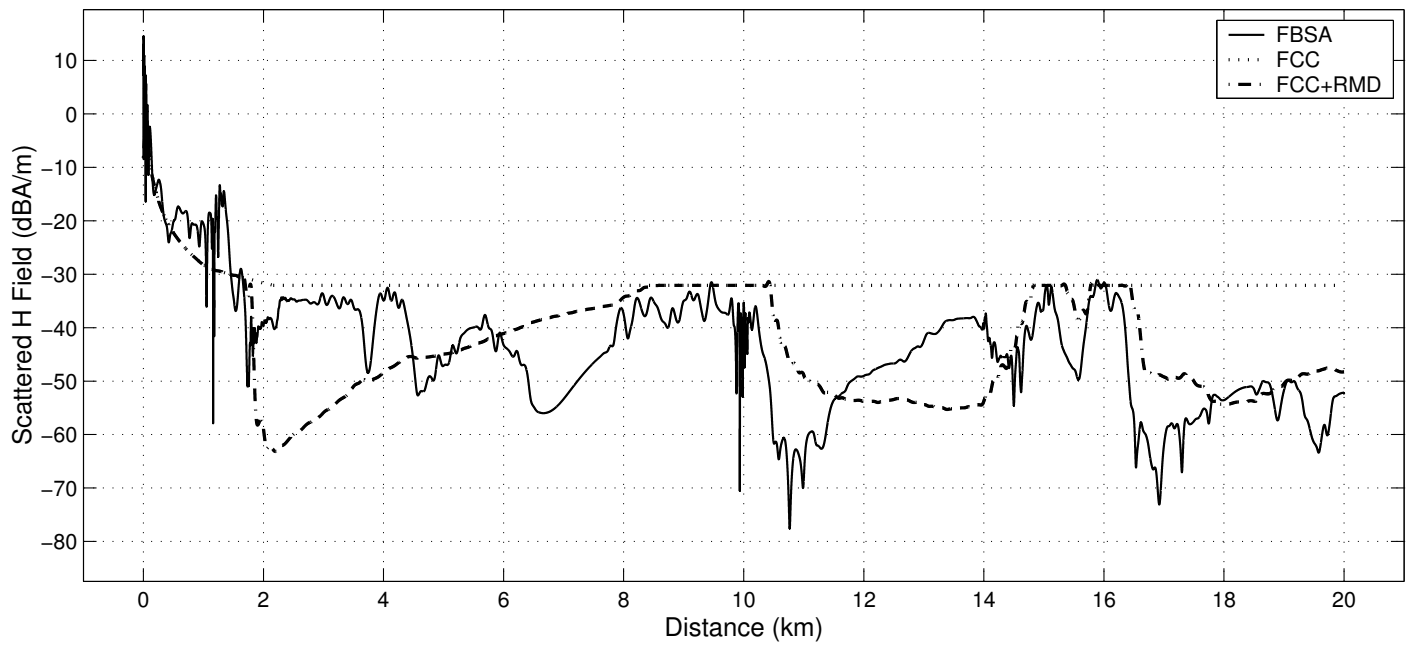

Figure 4.44: FCC vs. IE over uphill terrain for $890 \mathrm{MHz}$. 


\begin{tabular}{|c|c|c|c|c|}
\hline Absolute Error (\%) & DH (TM) & DH (TE) & UH (TM) & UH (TE) \\
\hline FreeSpace & 22.69 & 19.40 & 26.12 & 18.61 \\
\hline ITUR-370 & 43.79 & 40.39 & 75.94 & 62.94 \\
\hline ITUR-529 & 25.09 & 20.99 & 48.08 & 36.93 \\
\hline ITUR-1546 & 64.88 & 62.45 & 98.93 & 86.51 \\
\hline FCC & 42.74 & 42.00 & 32.02 & 35.05 \\
\hline FreeSpace + EpsPet & 18.50 & 15.56 & 46.66 & 38.23 \\
\hline ITUR-370 + EpsPet & 53.03 & 50.70 & 98.99 & 86.01 \\
\hline ITUR-529 + EpsPet & 31.75 & 29.21 & 73.26 & 62.22 \\
\hline ITUR-1546 + EpsPet & 78.99 & 77.14 & 126.8 & 113.5 \\
\hline FCC + EpsPet & 26.07 & 25.74 & 24.08 & 24.74 \\
\hline
\end{tabular}

Table 4.6: Absolute error values for $890 \mathrm{MHz}$

For the downhill profile analyzed, the right-most end point becomes in the asymptotic lit region of the source which is located 20 meters above the terrain at the left-most end. Hence, the free space propagation model with multiple diffraction corrections seems to be the best propagation model. Also, choosing the source as an isotropic radiator makes the free space propagation loss to be the best choice among models because of the $1 / d$ order in magnitudes of both field strengths. It is observed for the uphill geometry that, since this geometry is rougher than the downhill profile, the most preferable choice of the propagation model may change. Therefore, the best choice depends on the roughness of the surface, the operation frequency and the radiation pattern of the source. However it can be said that, for an isotropic radiator, the free space propagation loss and FCC curves are more preferable than the other propagation models. The worst results are obtained by the newest ITU recommendation ITUR-1546 for all frequency and polarization cases. In order to find out the best choice for the multiple diffraction correction methods, the FBSA solution is used as a reference. The field strength predictions are evaluated using Free Space Propagation model and multiple diffraction correction methods Bullington, Deygout, Epstein-Peterson and Vogler are compared. Figures 4.45-4.47 show these comparisons over the downhill profile, while Figures 4.48-4.50 illustrate examinations over the uphill one; for the TM and TE polarization for the three sets of frequencies. 
(a) non-PEC Terrain Profile

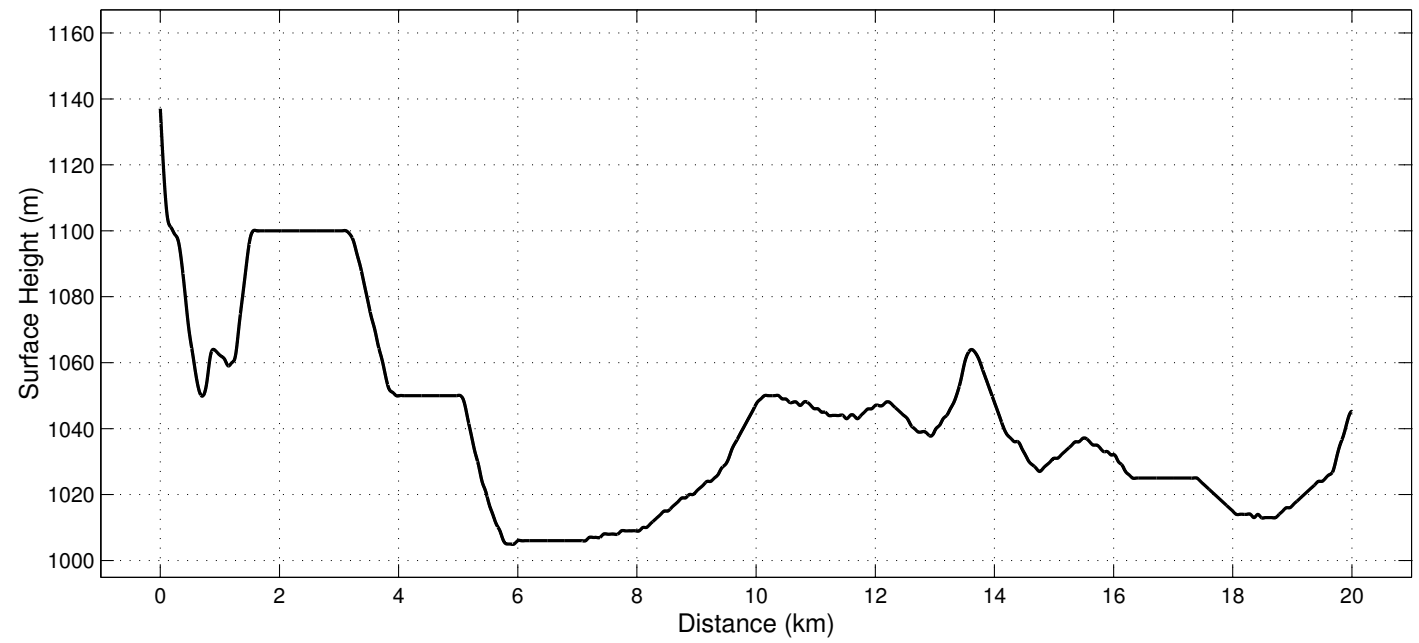

(b) TM Polarized Isotropic Radiator

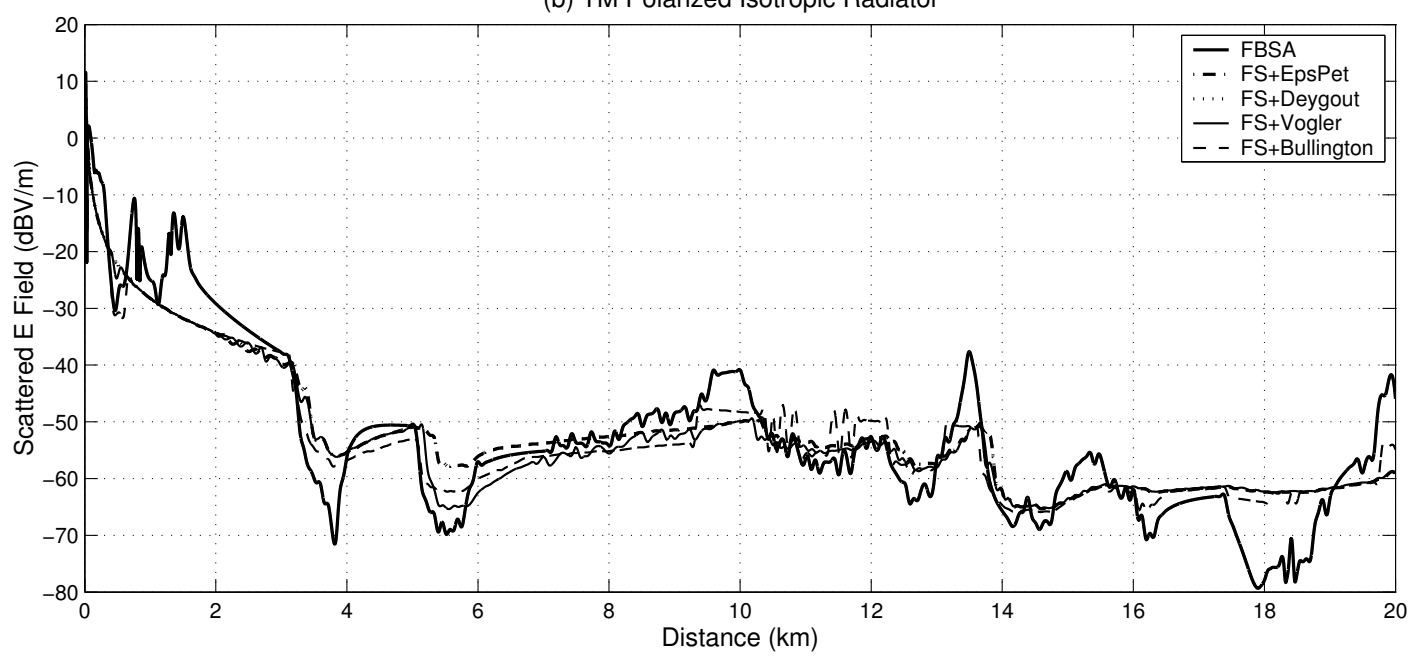

(c) TE Polarized Isotropic Radiator

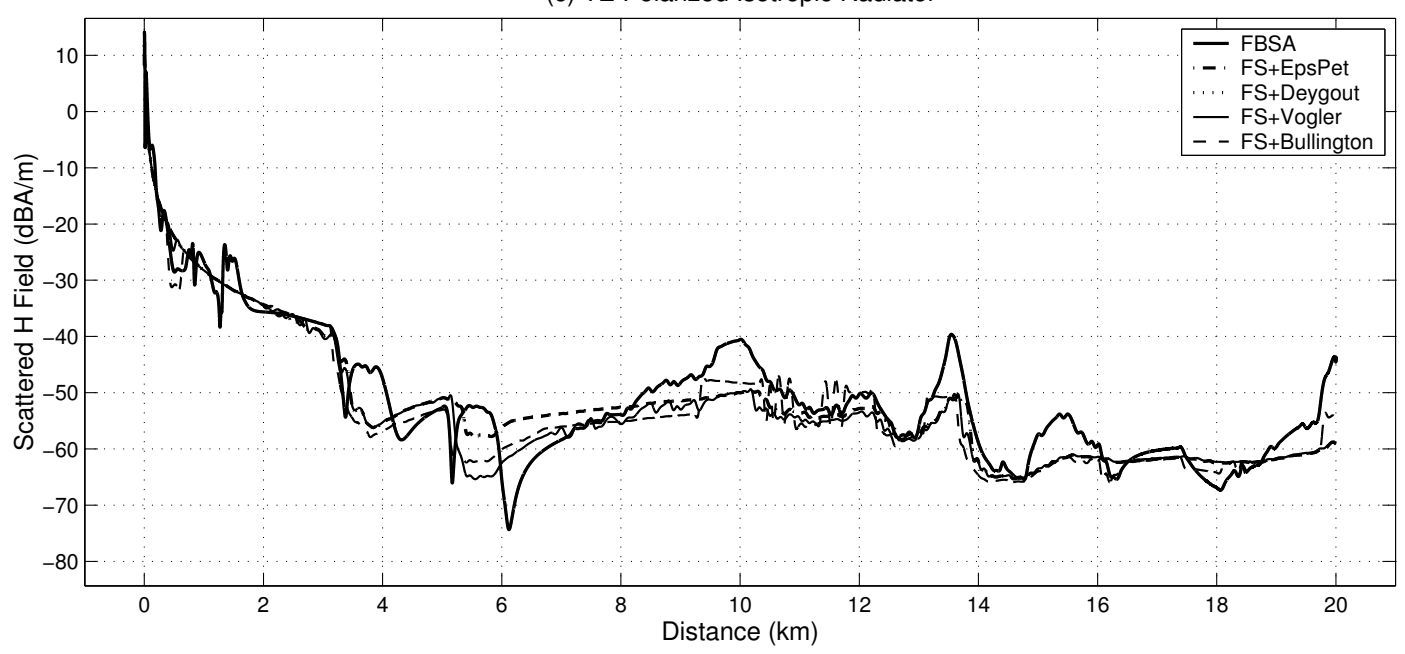

Figure 4.45: MD corrections vs. IE over downhill terrain for $200 \mathrm{MHz}$. 
(a) non-PEC Terrain Profile

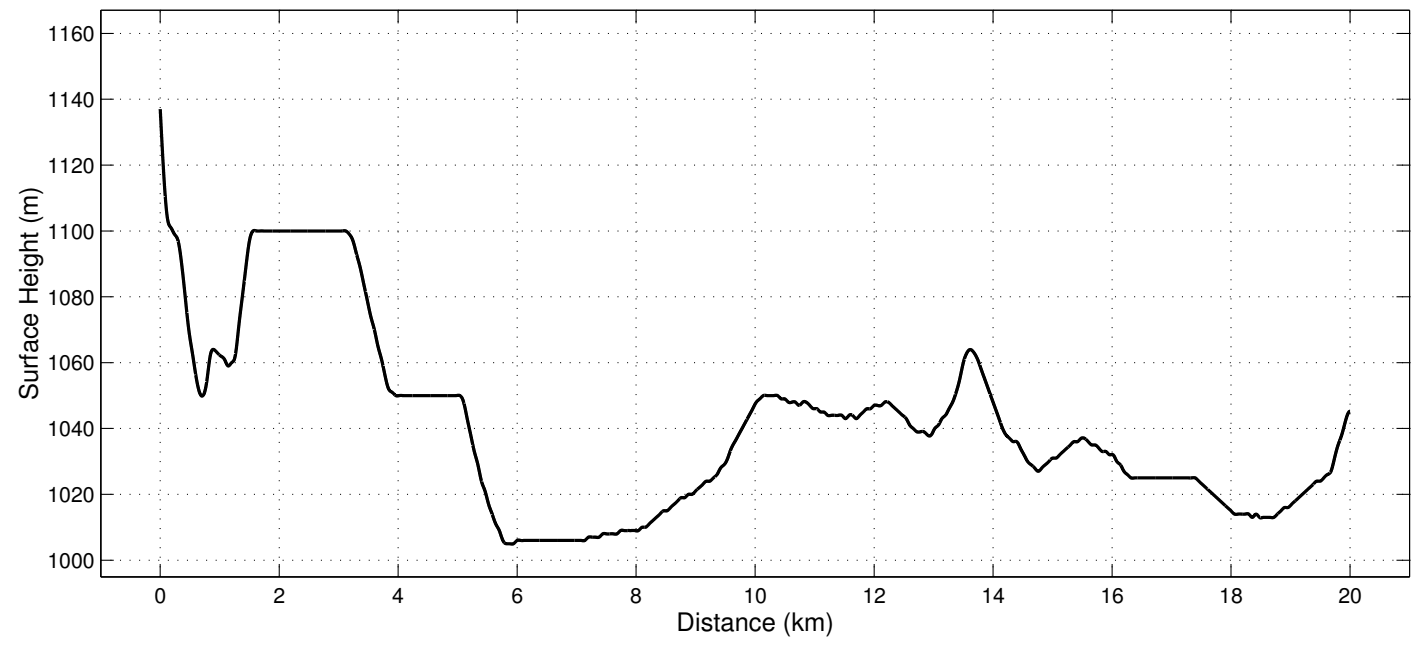

(b) TM Polarized Isotropic Radiator

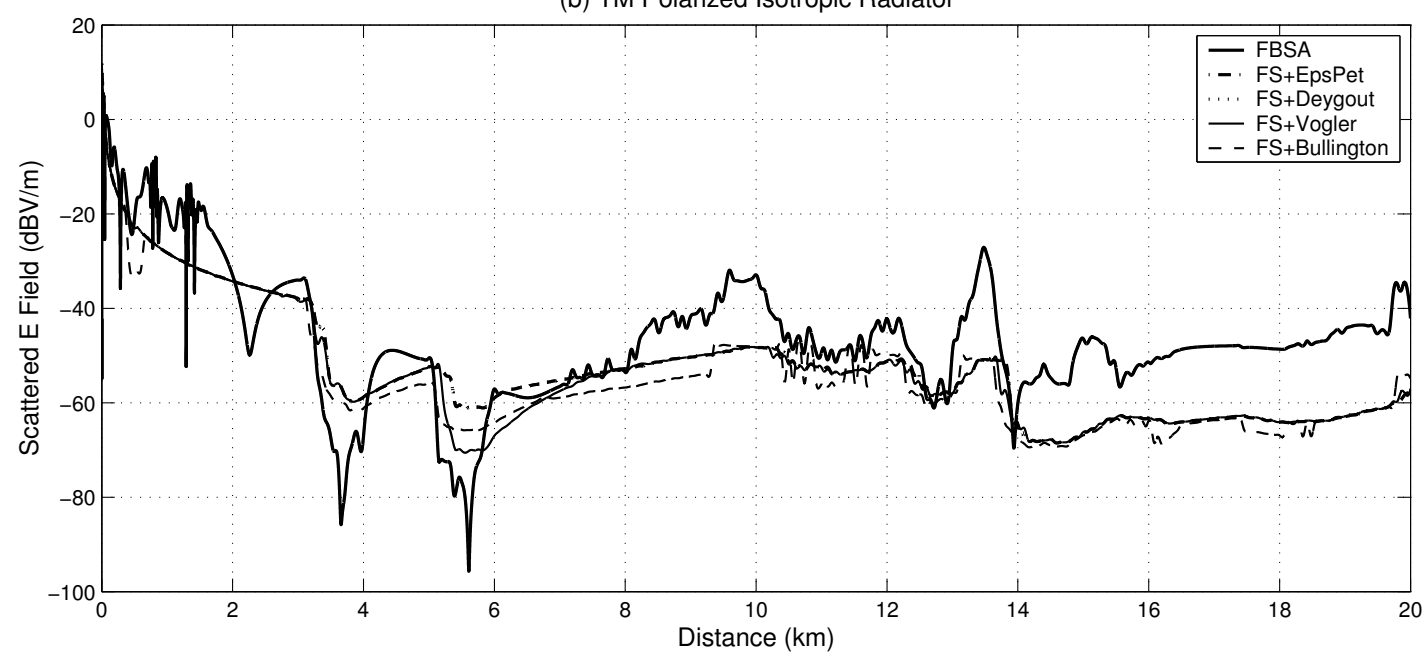

(c) TE Polarized Isotropic Radiator

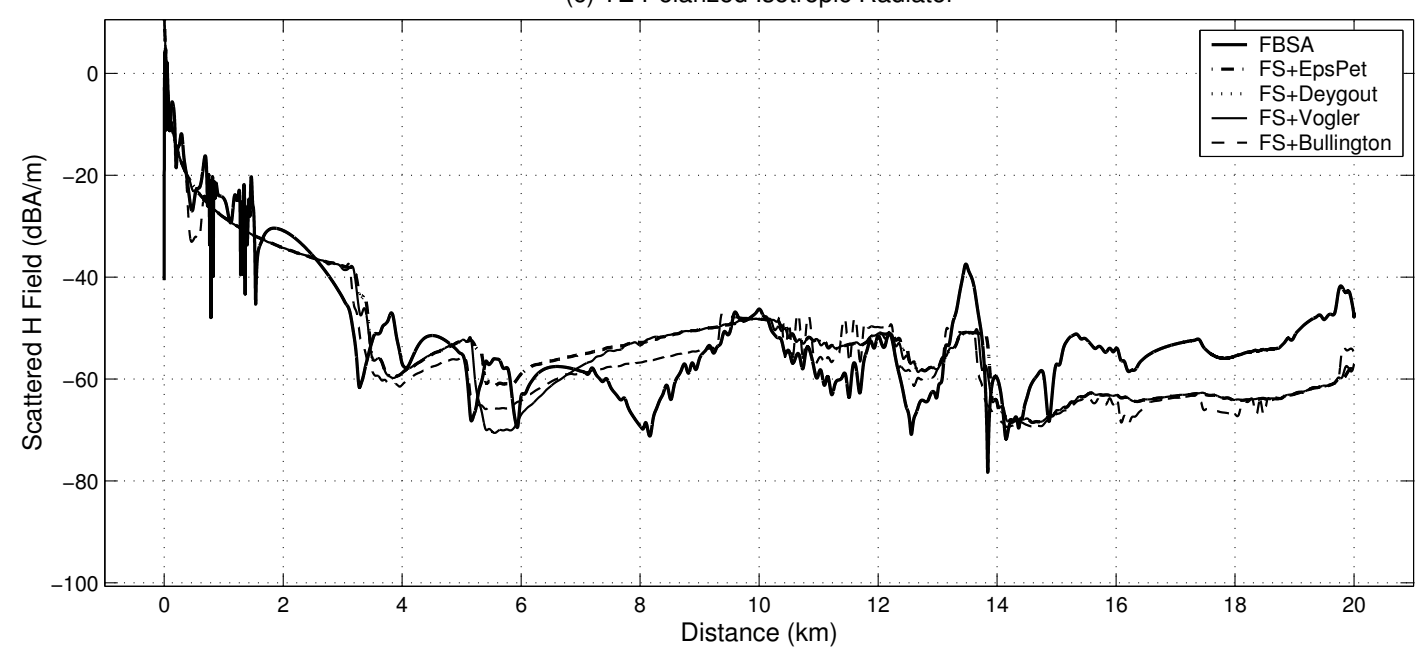

Figure 4.46: MD corrections vs. IE over downhill terrain for $500 \mathrm{MHz}$. 
(a) non-PEC Terrain Profile

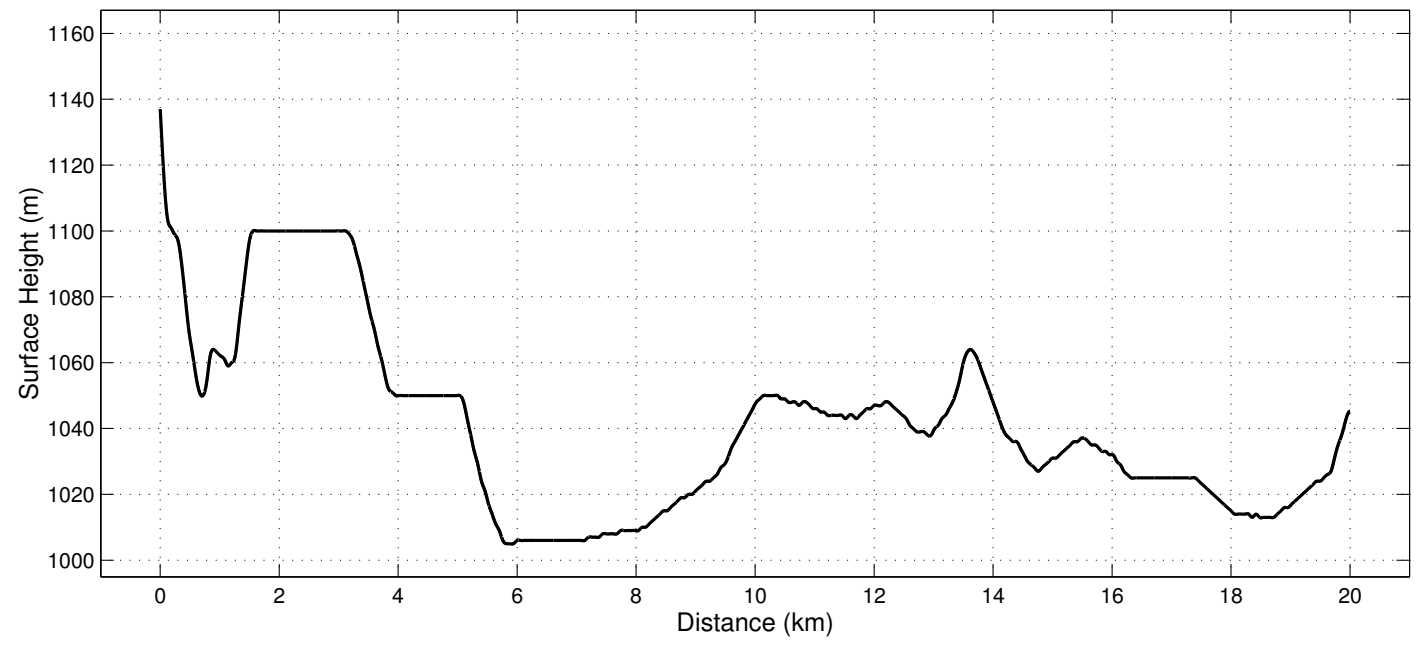

(b) TM Polarized Isotropic Radiator

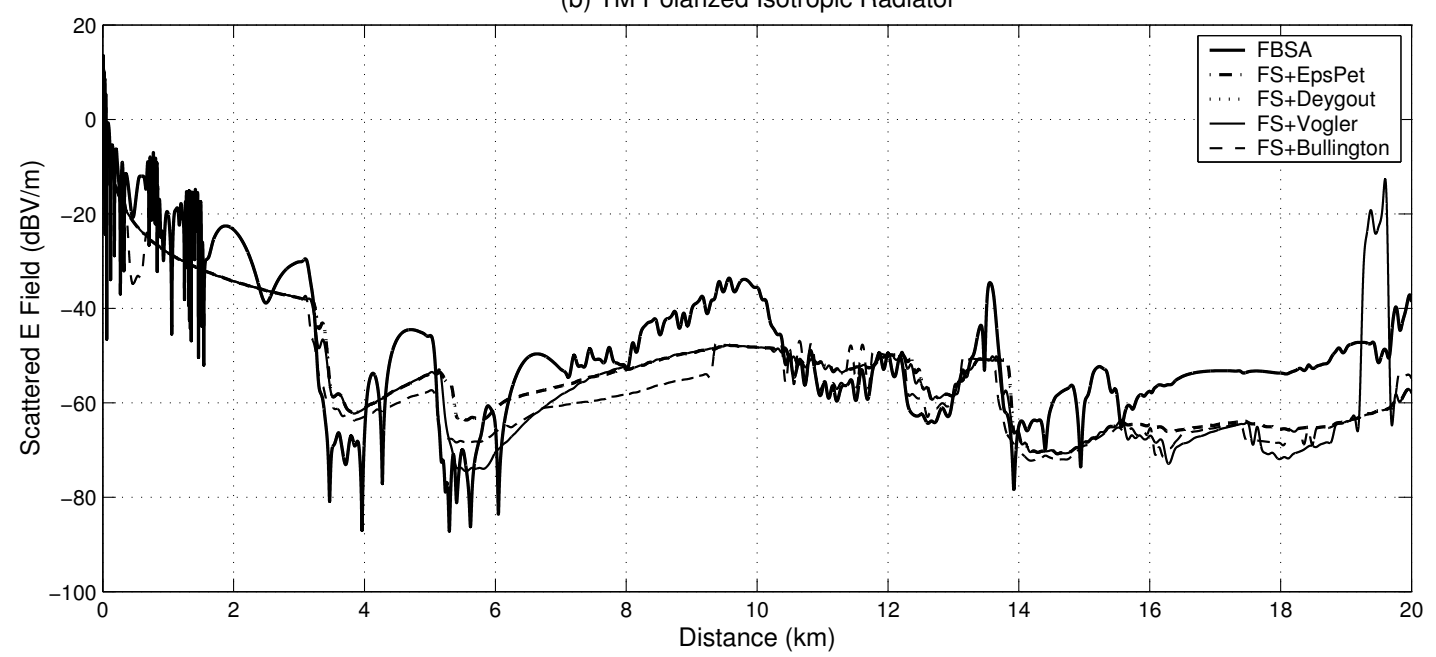

(c) TE Polarized Isotropic Radiator

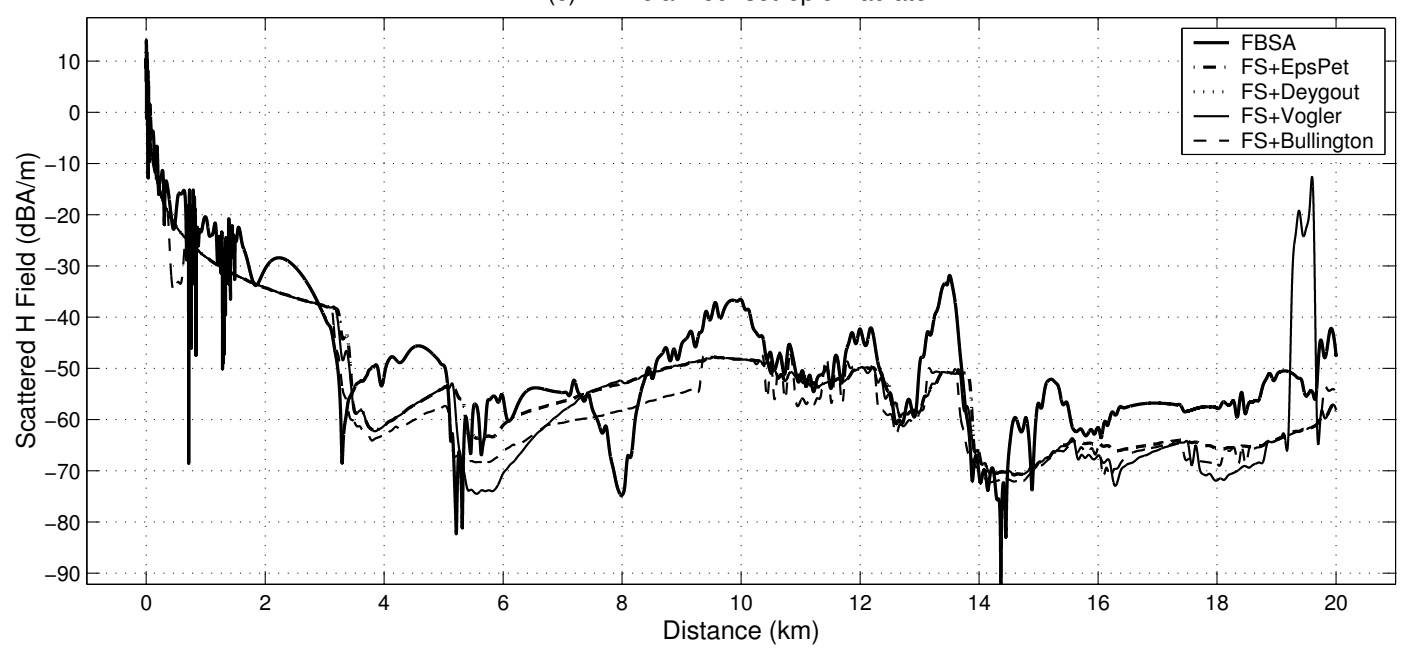

Figure 4.47: MD corrections vs. IE over downhill terrain for $890 \mathrm{MHz}$. 
(a) non-PEC Terrain Profile

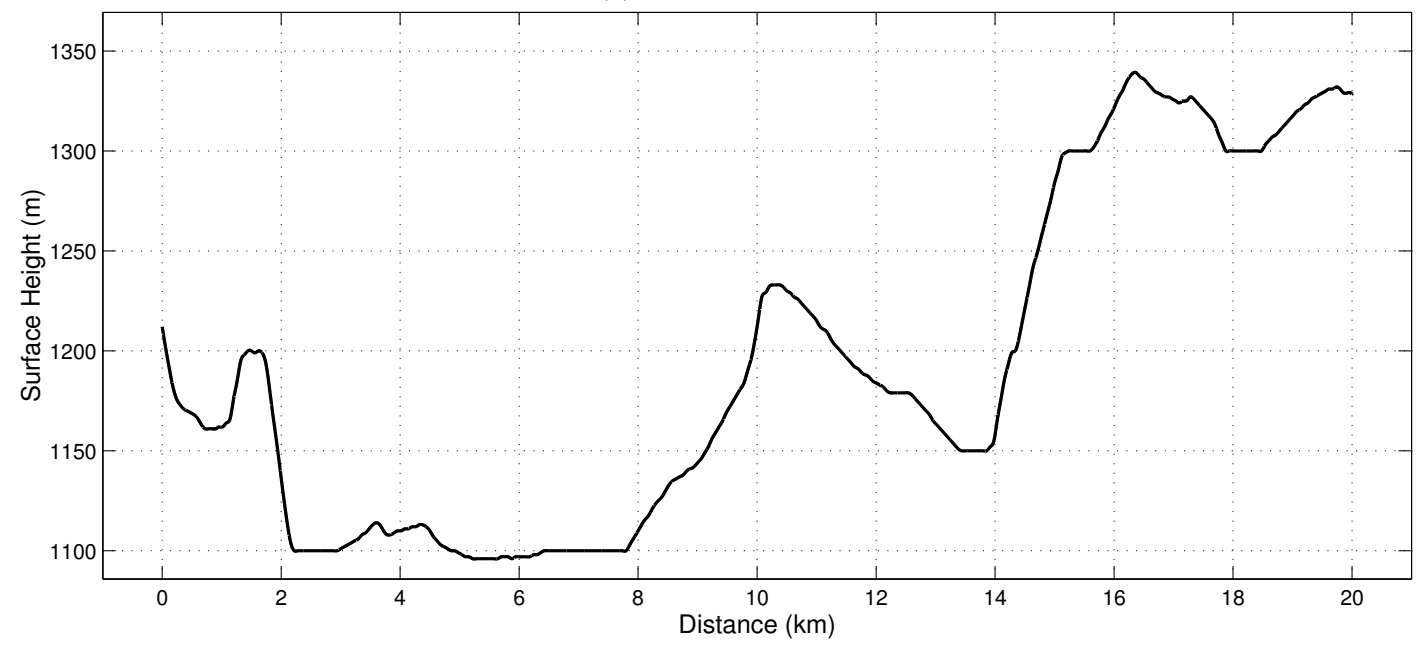

(b) TM Polarized Isotropic Radiator

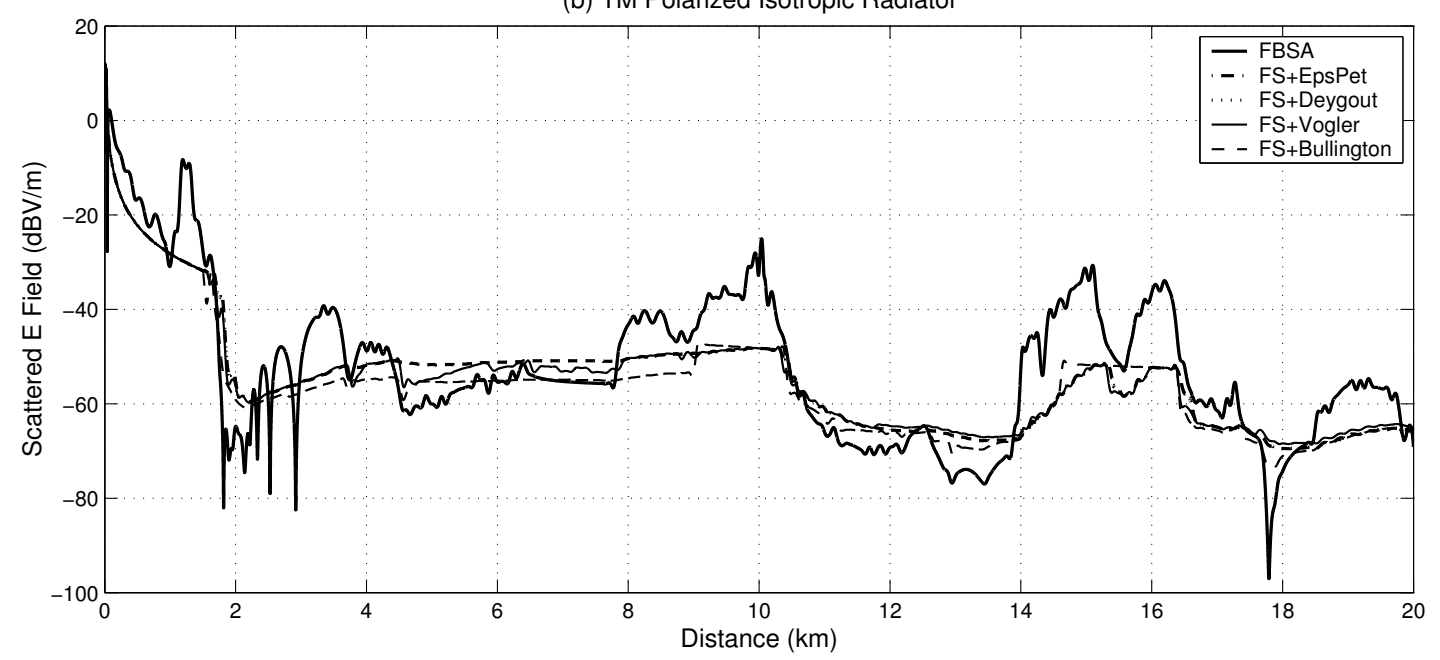

(c) TE Polarized Isotropic Radiator

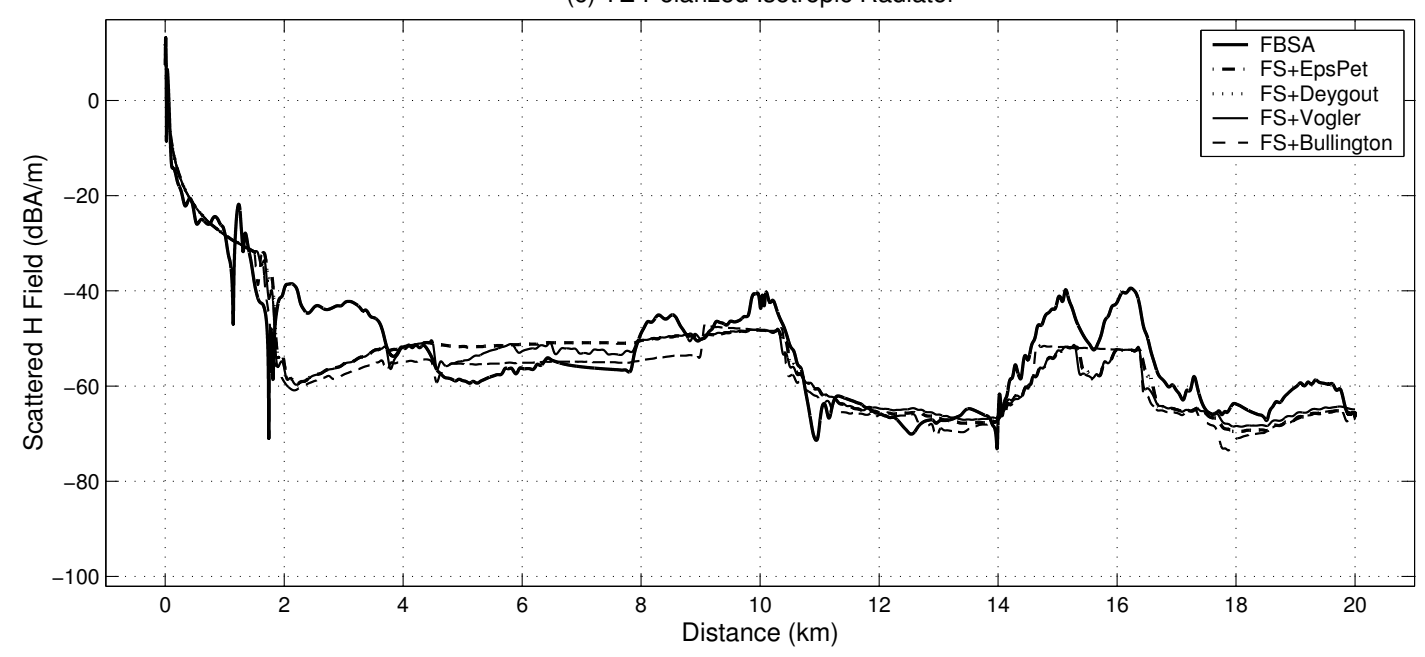

Figure 4.48: MD corrections vs. IE over uphill terrain for $200 \mathrm{MHz}$. 
(a) non-PEC Terrain Profile

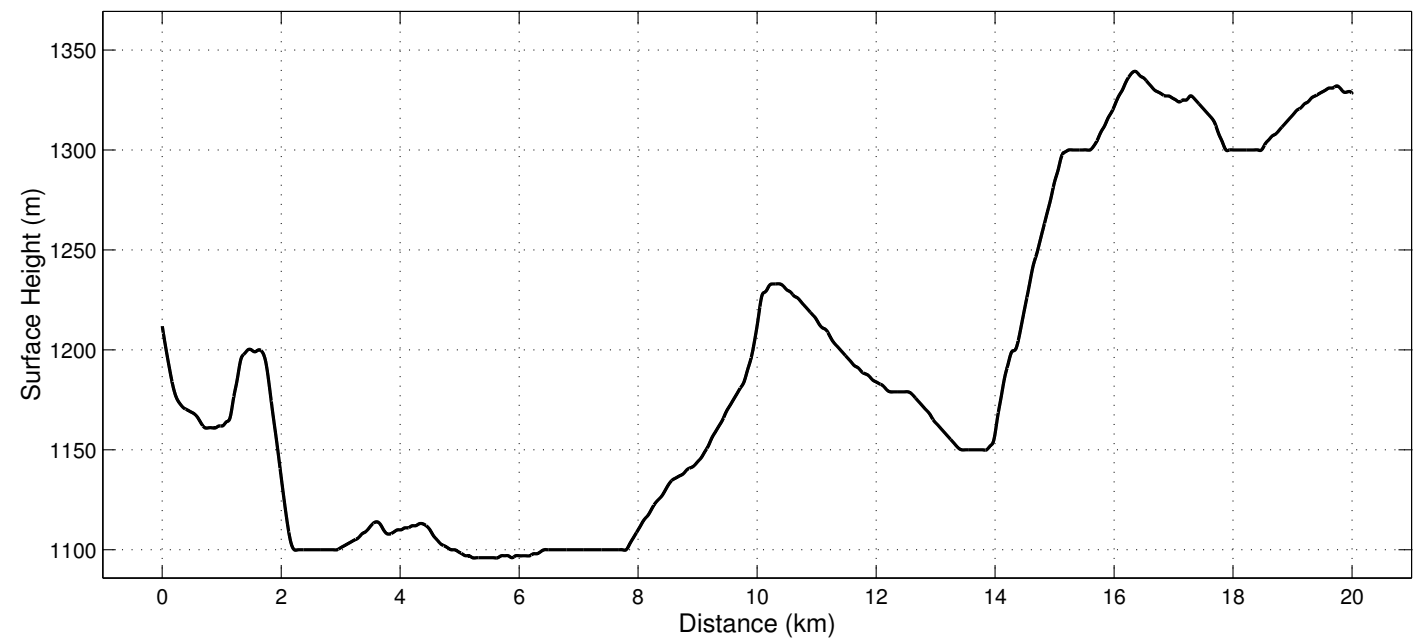

(b) TM Polarized Isotropic Radiator

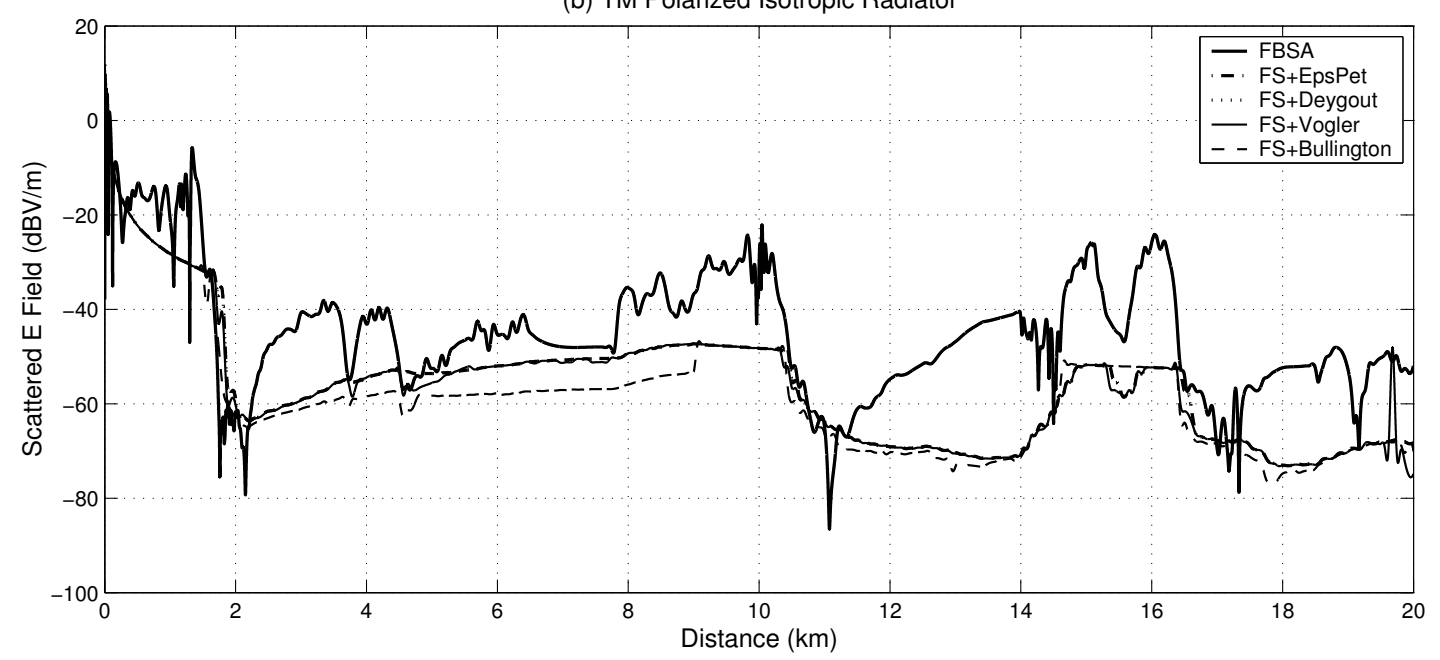

(c) TE Polarized Isotropic Radiator

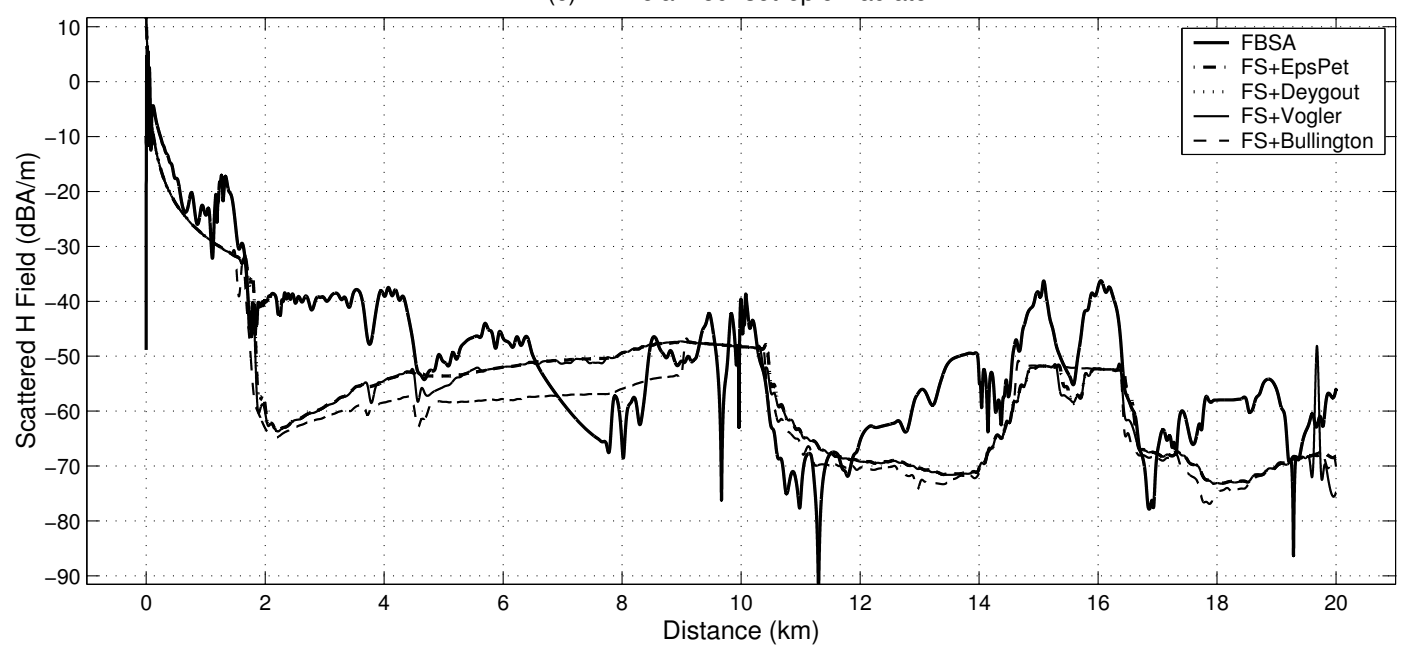

Figure 4.49: MD corrections vs. IE over uphill terrain for $500 \mathrm{MHz}$. 
(a) non-PEC Terrain Profile

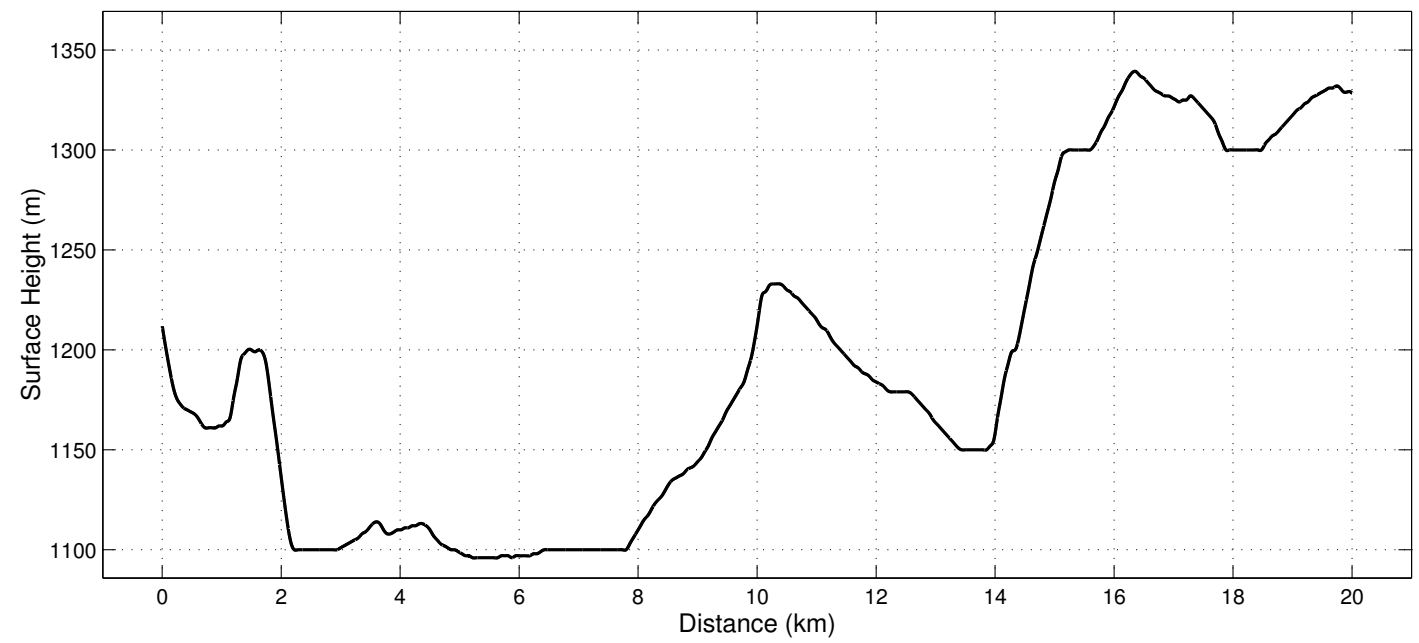

(b) TM Polarized Isotropic Radiator

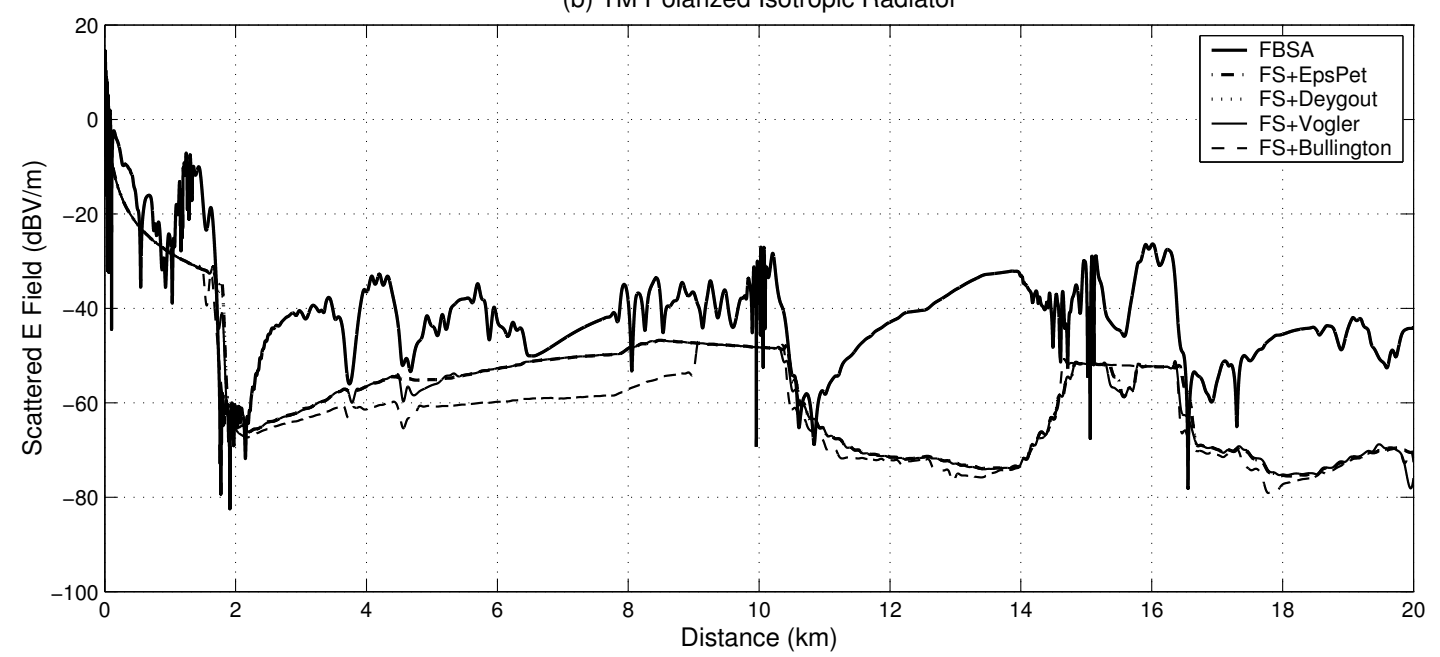

(c) TE Polarized Isotropic Radiator

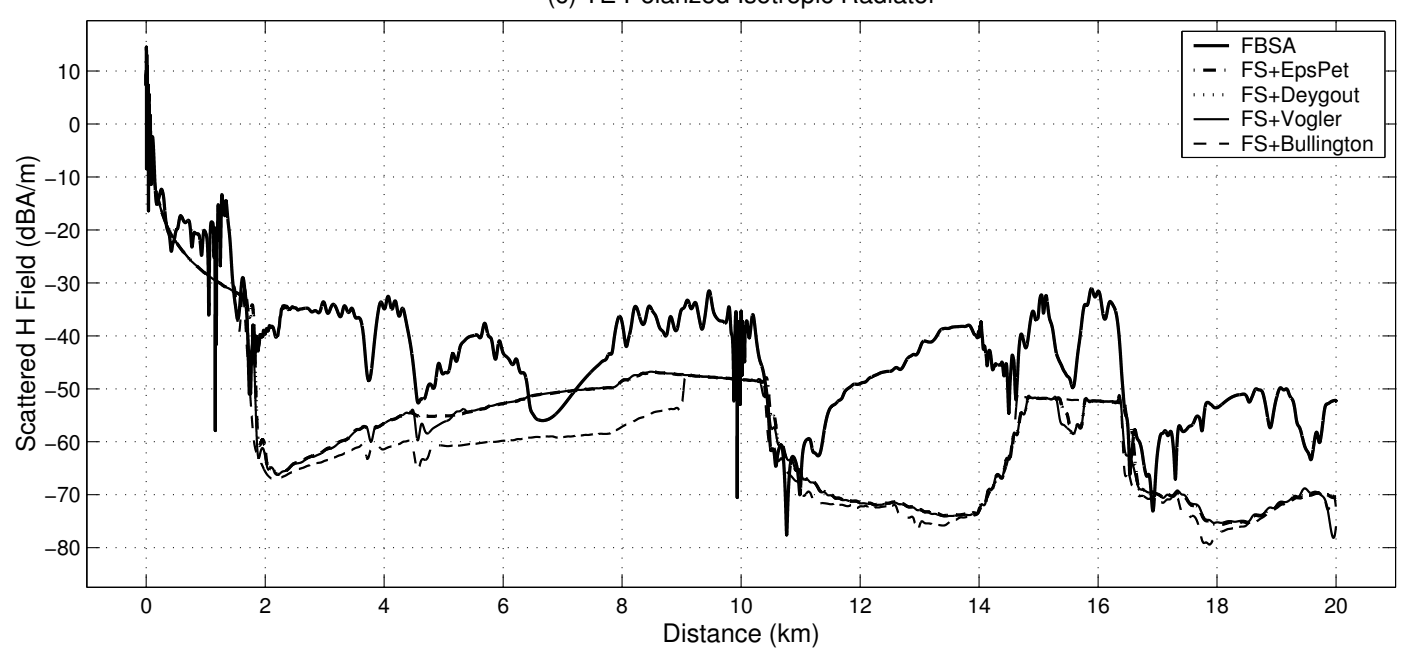

Figure 4.50: MD corrections vs. IE over uphill terrain for $890 \mathrm{MHz}$. 
The absolute error is used as the accuracy criterion to compare the multiple diffraction corrections with the FBSA . Tables 4.7 and 4.8 show the absolute error values for different correction models and polarizations over uphill and downhill profiles, respectively; for three sets of frequencies.

\begin{tabular}{|c|c|c|c|c|}
\hline Abs. Err. (\%) & EpsPet & Deygout & Vogler & Bullington \\
\hline $200 \mathrm{MHz}(\mathrm{TM})$ & 11.61 & 11.61 & 11.07 & 10.15 \\
\hline $200 \mathrm{MHz}(\mathrm{TE})$ & 9.22 & 9.22 & 9.38 & 9.02 \\
\hline $500 \mathrm{MHz}(\mathrm{TM})$ & 23.73 & 23.73 & 23.05 & 24.28 \\
\hline $500 \mathrm{MHz}(\mathrm{TE})$ & 14.62 & 14.62 & 14.66 & 14.06 \\
\hline $890 \mathrm{MHz}(\mathrm{TM})$ & 18.50 & 18.50 & 20.52 & 19.94 \\
\hline $890 \mathrm{MHz}(\mathrm{TE})$ & 15.56 & 15.56 & 19.36 & 16.72 \\
\hline
\end{tabular}

Table 4.7: Absolute error values for downhill profile

\begin{tabular}{|c|c|c|c|c|}
\hline Abs. Err. (\%) & EpsPet & Deygout & Vogler & Bullington \\
\hline $200 \mathrm{MHz}(\mathrm{TM})$ & 16.69 & 16.69 & 16.33 & 15.82 \\
\hline $200 \mathrm{MHz}(\mathrm{TE})$ & 12.03 & 12.03 & 11.59 & 12.16 \\
\hline $500 \mathrm{MHz}(\mathrm{TM})$ & 31.66 & 31.66 & 31.80 & 34.44 \\
\hline $500 \mathrm{MHz}(\mathrm{TE})$ & 20.83 & 20.83 & 21.11 & 22.39 \\
\hline $890 \mathrm{MHz}(\mathrm{TM})$ & 46.66 & 46.66 & 46.82 & 50.90 \\
\hline $890 \mathrm{MHz}(\mathrm{TE})$ & 38.23 & 38.23 & 38.46 & 42.45 \\
\hline
\end{tabular}

Table 4.8: Absolute error values for uphill profile

It is observed that the largest multiple diffraction loss is obtained by the Bullington method, and the least by the Epstein-Peterson or Deygout. So, if the reference solution of the field strength is larger than the propagation model in magnitude, Bullington yields better results than others. However, inspecting the figures it is obvious that, the Bullington method cannot catch the diffraction loss effects of some of the consecutive peaks that are close to each other. Therefore, the best choice for the multiple diffraction correction seems to be Epstein-Peterson or Deygout. Note that, all results for Epstein-Peterson and Deygout methods are identical.

For examination of the computational cost of the reference solution obtained by FBSA, Table 4.9 is given. The CPU-time values are obtained for the studies of the method on a Intel PIII microprocessor having a $500 \mathrm{MHz}$ clock frequency. CPU-time results show that the computational cost is very dependent on the 


\begin{tabular}{|c|c|c|}
\hline Number of Unknowns $(N)$ & Num. of integr. points & CPU-time/iter (s) \\
\hline 133333 (Downhill) & 225 & 233 \\
\hline 333333 (Downhill) & 286 & 903 \\
\hline 593333 (Downhill) & 346 & 2425 \\
\hline 133333 (Uphill) & 286 & 265 \\
\hline 333333 (Uphill) & 366 & 1073 \\
\hline 593333 (Uphill) & 439 & 2758 \\
\hline
\end{tabular}

Table 4.9: Computational Cost for the FBSA

number of integration points. However, it is obvious that increasing the number of surface unknowns for a fixed number of integral steps, the modified algorithm yields an $O(N)$ computational time. Since, the uphill geometry analyzed is rougher than the downhill one, the number of integration sampling points is larger.

It is also worth mentioning that, the empirical methods are run on the surfaces for 30 meters long segments, since the computer tool developed by Communications and Spectrum Management Research Center uses the real-life terrain profiles and takes the surface data from Digital Terrain Elevation Data (DTED) maps which are based on Geographical Information Systems (GIS) information. Therefore, these methods are very fast to apply and give the field strength results in one second for a $20 \mathrm{~km}$ length surface profile. 


\section{Chapter 5}

\section{CONCLUSIONS}

In this work, a great number of implementations of conventional FBM and FBSA over various kinds of rough surface profiles have been presented in order to examine the most common propagation prediction models and multiple diffraction loss correction methods, and find out the most preferable ones.

In order to compare the FBM results with the previous work in the literature, examinations over one-dimensional strip profiles have been presented, first. Then, accuracy of the FBM has been tested with respect to the Method of Moments solution, over various kinds of rough surface profiles, for both TM and TE polarization cases.

The conventional FBM has been shown to be an accurate solution, with its very accurate solutions and rapid convergence ability, for large numbers of surface unknowns where MoM fails because of the operational cost. However, the computational cost of the FBM is still $O\left(N^{2}\right)$, which prevents to analyze electrically large rough surfaces.

The Spectral Acceleration Forward Backward Method has been applied over slightly rough surfaces for the computation of the scattering. Examinations of 
the FBSA with respect to MoM and FBM have been presented, and the FBSA has been shown to be used as a reference solution for scattering from extremely large rough surfaces, because of its accurate results within very few iterations and the computational cost of $O(N)$, where $N$ is the surface unknowns.

The conventional FBM solution has been used to test the accuracy of FBSA over rough surface profiles where MoM fails because of the large number of surface unknowns. With these comparisons, the limitations of the FBSA over both slightly rough surfaces and terrain profiles have been detected. The original spectral acceleration algorithm is good at analyzing slightly rough surfaces. Convergence problems occur as the roughness increases due to the selection of the inclination angle of the integration path and assumption of the origin to be the most effective saddle point. Thus, the original algorithm becomes not suitable for terrain profiles, and hence, modified FBSA method has been used to analyze propagation over terrain. However, a limitation for this algorithm has been found out during examinations. For very rough terrain profiles such that the inclination angle of the deformed integration contour becomes less than 4 degrees, the modified FBSA fails to produce accurate results.

The main novelty of this work is presenting examinations of propagation prediction models and multiple diffraction correction models, using modified FBSA as a reference solution for propagation over terrain. In the previous work, an important number of comparisons of integral equation methods based on 2D Green's function with measurement data or Hata model have been presented [18], [20]. However, those IE methods do not dicretize the surface profile as much as FBSA does and also they use additional approximations, such as neglecting the backscatter. Furthermore, none of them was used for examining the propagation models or diffraction loss methods.

In this work, a great number of implementations of the propagation models have been compared with the integral equation based accurate FBSA solutions 
over various terrain profiles for different frequency sets. During these examinations, 600 thousand surface unknowns for the computation of rough surface scattering have been reached. That much surface unknowns is more than twice of the maximum number in the previous work on one-dimensional rough surface scattering.

According to the examinations, the most preferable propagation models and diffraction correction methods have been proposed. Advantages and limitations of the most common propagation models have not been analyzed such a detailed way in the literature. It has been found out that most recent recommended propagation models generate much more error than relatively old, and even withdrawn, models do.

Propagation models and multiple diffraction correction methods do not depend on the polarization of the source. Also, they do not response to changes in electrical properties of terrain, such as relative permittivity and permeability. Furthermore, diffraction correction methods take only the loss effects of the diffraction into account, but not the constructive contributions. The total field attenuation for propagation models is the sum of the field attenuation of the empirical model, the reflection loss and the diffraction loss. Hence, using MDCs increases the error, if field strength values predicted by an empirical model are at a lower level in magnitude than the reference. All multiple diffraction corrections yield quiet similar results. Epstein-Peterson or Deygout can be considered to be the most preferable one.

Selecting the best propagation model depends on the type of the source, terrain profile and the frequency. If the total field is predicted generally in the lit region of an isotropic radiator, selecting Free Space Propagation Model is preferable. However, this model is not frequency dependent, thus it loses its validity as the frequency increases. The second best model seems to be FCC curves, especially for uphill geometries. The worst model for every type of frequency and 
terrain profile has been found to be the most recent recommendation of ITU, that is ITUR-1546.

Future work may imply the modifications of the spectral acceleration parameters in order to analyze rougher terrain profiles and proposing novel propagation models or diffraction corrections for propagation over terrain. 


\section{Appendix A}

\section{Spectral Acceleration for the Backward Propagation}

\section{A.1 Horizontal Polarization}

The FBM procedure of the electric field integral equation for horizontal polarization requires repeated computation of the matrix-vector products $\overline{\mathbf{Z}}^{f} \cdot \mathbf{I}$ and $\overline{\mathbf{Z}}^{b} \cdot \mathbf{I}$ as

$$
\begin{gathered}
E_{f}\left(\boldsymbol{\rho}_{n}\right)=\sum_{m=1}^{n-1} I_{m} Z_{n m} \\
E_{b}\left(\boldsymbol{\rho}_{n}\right)=\sum_{m=n+1}^{N} I_{m} Z_{n m}
\end{gathered}
$$

where $N$ is the number of surface unknowns. (A.1) and (A.2) denotes the forward and backward radiations of the source elements in front and at the rear of the $n$th receiving element, respectively.

The spectral acceleration of the forward propagation was described in Chapter 3. This appendix treats to the backward propagation in an analogous procedure. Selecting a neighborhood distance $L_{s}$ for a receiving element, within which interactions are classified as strong and outside of which interactions are classified as 
weak, the acceleration algorithm for computing (A.1) and (A.2) in $O(N)$ begins. As a result (A.2) can be rewritten as

$$
\begin{gathered}
E_{b}\left(\boldsymbol{\rho}_{n}\right)=E_{s}\left(\boldsymbol{\rho}_{n}\right)+E_{w}\left(\boldsymbol{\rho}_{n}\right) \\
=\sum_{m=n+N_{s}}^{n+1} I_{m} Z_{n m}+\sum_{m=N}^{n+N_{s}+1} I_{m} Z_{n m},
\end{gathered}
$$

where $N_{s}=L_{s} / \Delta x$ denotes the number of elements that have strong interactions with the $n$th element. The first term in (A.3) denoted by $E_{s}$ represents the strong region contributions and the second term denoted by $E_{w}$ represents the weak region contributions. The off-diagonal entries of the impedance matrix for horizontal polarization were derived using the EFIE for non-PEC surfaces as

$$
Z_{n m}=-j \omega \mu G\left(\boldsymbol{\rho}_{n}, \boldsymbol{\rho}_{m}\right) \Delta x_{m}+\eta_{m} \Delta x_{m} \frac{\partial}{\partial n_{m}} G\left(\boldsymbol{\rho}_{n}, \boldsymbol{\rho}_{m}\right)
$$

The strong region contributions $E_{s}$ are found in the conventional FBM manner using the exact matrix elements, while the rapid computation of the weak region contributions $E_{w}$ is performed by employing the spectral representation of the two-dimensional Green's function and its partial derivative. The spectral representation of the Green's function is expressed as:

$$
G\left(\boldsymbol{\rho}_{n}, \boldsymbol{\rho}_{m}\right)=\frac{-j}{4 \pi} \int_{C_{\phi}} e^{-j k\left[\left(x_{n}-x_{m}\right) \cos \phi+\left(z_{n}-z_{m}\right) \sin \phi\right]} d \phi
$$

where $C_{\phi}$ is the contour of integration in the complex $\phi$ space. On the other hand, the spectral representation of the partial derivative of the Green's function with respect to the normal vector on source point can be expressed as:

$$
\begin{aligned}
\frac{\partial}{\partial n_{m}} G\left(\boldsymbol{\rho}_{n}, \boldsymbol{\rho}_{m}\right)= & \frac{k}{4 \pi} \int_{C_{\phi}}\left[\cos \theta_{m} \cos \phi+\sin \theta_{m} \sin \phi\right] \\
& \cdot e^{-j k\left[\left(x_{n}-x_{m}\right) \cos \phi+\left(z_{n}-z_{m}\right) \sin \phi\right]} d \phi
\end{aligned}
$$

where $\theta_{m}$ is the angle between the unit normal vector to the surface at the source point, $\hat{n}_{m}$, and the unit vector in the $-x$ direction, $-\hat{x}$.

Substituting (A.5) and (A.6) into (A.3) and interchanging the integration and summation, yields to:

$$
E_{w}\left(\boldsymbol{\rho}_{n}\right)=-\frac{\omega \mu}{4 \pi} \int_{C_{\phi}} F_{n}(\phi) d \phi
$$


where

$$
\begin{aligned}
F_{n}(\phi)= & \sum_{m \in G_{w}} I_{m} \Delta x_{m}\left\{1-\frac{\eta_{m}}{\eta_{o}}\left[\cos \theta_{m} \cos \phi+\sin \theta_{m} \sin \phi\right]\right\} \\
& \cdot e^{-j k\left[\left(x_{n}-x_{m}\right) \cos \phi+\left(z_{n}-z_{m}\right) \sin \phi\right]}
\end{aligned}
$$

showing that weak element contributions at point $\boldsymbol{\rho}_{n}$ can be obtained through a spectral domain integral of the weak element complex far-field pattern or plane wave spectrum, $F_{n}(\phi)$. It is at this point where the great reduction in computational cost is obtained because $F_{n}(\phi)$ can be easily updated using a recursive procedure. In general, $F_{n}(\phi)$ can be expressed as a function of $F_{n+1}(\phi)$ as follows [7]:

$$
\begin{aligned}
F_{n}(\phi)= & F_{n+1}(\phi) e^{-j k\left[\left(x_{n}-x_{n+1}\right) \cos \phi+\left(z_{n}-z_{n+1}\right) \sin \phi\right]} \\
& +I_{n s} \Delta x_{n s}\left\{1-\frac{\eta_{n s}}{\eta_{o}}\left[\cos \theta_{n s} \cos \phi+\sin \theta_{n s} \sin \phi\right]\right\} \\
& \cdot e^{-j k\left[\left(x_{n}-x_{n s}\right) \cos \phi+\left(z_{n}-z_{n s}\right) \sin \phi\right]}
\end{aligned}
$$

with $F_{n}(\phi)=0$ for $n \geq N-N_{s}$ in the backward sweep. In (A.9), $n s=n+N_{s}+1$ is the new source point introduced in the weak group as the iterative procedure sweeps the surface in the backward direction. Namely, (A.9) shows that $F_{n}(\phi)$ continuously updates as each new source element enters the weak interaction group in the backward sweep, and hence, the complex far field pattern at the receiving element is expressed in terms of that at the next one. This procedure produces an $O(N)$ operational cost.

\section{A.2 Vertical Polarization}

Similar to the horizontal polarization case, when using the magnetic field integral equation in the vertical polarization case, the radiating elements are also divided into two groups: the strong interactions region $G_{s}$, and the weak interactions region $G_{w}$. And their contribution to the backward propagating magnetic field 
$H_{b}$ can be expressed as follows:

$$
\begin{gathered}
H_{b}\left(\boldsymbol{\rho}_{n}\right)=H_{s}\left(\boldsymbol{\rho}_{n}\right)+H_{w}\left(\boldsymbol{\rho}_{n}\right) \\
=\sum_{m=n+N_{s}}^{n+1} I_{m} Z_{n m}+\sum_{m=N}^{n+N_{s}+1} I_{m} Z_{n m},
\end{gathered}
$$

The off-diagonal entries of the impedance matrix for vertical polarization were derived using the MFIE for non-PEC surfaces as

$$
Z_{n m}=j \omega \epsilon \eta_{m} \Delta x_{m} G\left(\boldsymbol{\rho}_{n}, \boldsymbol{\rho}_{m}\right)-\Delta x_{m} \frac{\partial}{\partial n_{m}} G\left(\boldsymbol{\rho}_{n}, \boldsymbol{\rho}_{m}\right) .
$$

The strong region contributions $H_{s}$ are found in the conventional FBM manner using the exact matrix elements, while the rapid computation of the weak region contributions $H_{w}$ is performed by employing the spectral representation of the two-dimensional Green's function and its partial derivative.

Substituting the spectral representation of the two-dimensional Green's function, (A.5), and its partial derivative with respect to the unit normal vector to the surface on the source location, (A.6), into (A.10) and interchanging the integration and summation, yields to:

$$
H_{w}\left(\boldsymbol{\rho}_{n}\right)=-\frac{k}{4 \pi} \int_{C_{\phi}} F_{n}(\phi) d \phi
$$

where

$$
\begin{aligned}
F_{n}(\phi)= & \sum_{m \in G_{w}} I_{m} \Delta x_{m}\left\{\cos \theta_{m} \cos \phi+\sin \theta_{m} \sin \phi-\frac{\eta_{m}}{\eta_{o}}\right\} \\
& \cdot e^{-j k\left[\left(x_{n}-x_{m}\right) \cos \phi+\left(z_{n}-z_{m}\right) \sin \phi\right]}
\end{aligned}
$$

showing that weak element contributions at point $\boldsymbol{\rho}_{n}$ can be obtained through a spectral domain integral of the weak element complex far-field pattern or plane wave spectrum, $F_{n}(\phi) . F_{n}(\phi)$ can be easily updated using a recursive procedure. In general, $F_{n}(\phi)$ can be expressed as a function of $F_{n+1}(\phi)$ as follows.

$$
\begin{aligned}
F_{n}(\phi)= & F_{n+1}(\phi) e^{-j k\left[\left(x_{n}-x_{n+1}\right) \cos \phi+\left(z_{n}-z_{n+1}\right) \sin \phi\right]} \\
& +I_{n s} \Delta x_{n s}\left\{\cos \theta_{n s} \cos \phi+\sin \theta_{n s} \sin \phi-\frac{\eta_{n s}}{\eta_{o}}\right\} \\
& \cdot e^{-j k\left[\left(x_{n}-x_{n s}\right) \cos \phi+\left(z_{n}-z_{n s}\right) \sin \phi\right]}
\end{aligned}
$$


with $F_{n}(\phi)=0$ for $n \geq N-N_{s}$ in the backward sweep. In (A.14), $n s=n+N_{s}+1$ is the new source point introduced in the weak group as the iterative procedure sweeps the surface in the backward direction. Namely, (A.14) shows that $F_{n}(\phi)$ continuously updates as each new source element enters the weak interaction group in the backward sweep, and hence, the complex far field pattern at the receiving element is expressed in terms of that at the next one. This procedure produces an $O(N)$ operational cost. 


\section{Bibliography}

[1] "Special issue on low-grazing-angle backscattering from rough surfaces", IEEE Trans. Antennas and Propagat., vol. 46, Jan. 1998.

[2] R. F. Harrington, Field Computation by Moment Method, New York: IEEE Press, 1993.

[3] D. Holliday, L. L. DeRaad Jr., and G. J. St.-Cyr, "Volterra approximation for low grazing angle shadowing ocean-like surfaces", IEEE Trans. Antennas and Propagat., vol. 43, pp. 1199-1206, Nov. 1995.

[4] D. Holliday, L. L. DeRaad Jr., and G. J. St.-Cyr, "Forward-Backward: A new method for computing low-grazing scattering", IEEE Trans. Antennas and Propagat., vol. 44, pp. 722-729, May 1996.

[5] D. A. Kapp and G. S. Brown, "A new numerical method for rough surface scattering calculations", IEEE Trans. Antennas and Propagat., vol. 44, pp. 711-721, May 1996.

[6] H.-T. Chou and J. T. Johnson, "Novel multipole acceleration of forwardbackward method for scattering from rough surfaces", IEEE Antennas and Propagation Society International Symposium, vol. 1, pp. 100-103, Jun. 1998.

[7] H.-T. Chou and J. T. Johnson, "A novel acceleration for the computation of scattering from rough surfaces with the forward-backward method", Radio Sci., vol. 33, pp. 1277-1287, Jun. 1998. 
[8] "VHF and UHF propagation curves for the frequency range from $30 \mathrm{MHz}$ to $1000 \mathrm{MHz",} \mathrm{Recommendation} \mathrm{ITU-R} \mathrm{P.370-7,} 1995$.

[9] "Prediction methods for the terrestrial land mobile service in the VHF and UHF bands", Recommendation ITU-R P.529-3, 1999.

[10] "Method for point-to-area predictions for terrestrial services in the frequency range $30 \mathrm{MHz}$ to $3000 \mathrm{MHz}$ ", Recommendation ITU-R P.1546, 2001.

[11] "Federal Communications Commission Curves", www.fcc.gov.

[12] C. L. Rino and H. D. Ngo, "Forward propagation in half-space with an irregular boundary", IEEE Trans. Antennas and Propagat., vol. 45, pp. 1340-1347, Sept. 1997.

[13] D. J. Donohue and J. R. Kuttler, "Propagation modeling over terrain using the parabolic wave equation", IEEE Trans. Antennas and Propagat., vol. 48, pp. 260-277, Feb. 2000.

[14] A. E. Barrios, "A terrain parabolic equation model for propagation in the troposphere", IEEE Trans. Antennas and Propagat., vol. 42, pp. 90-98, Jan. 1994.

[15] S. Ayasli, "SEKE: A computer model for low altitude radar propagation over irregular terrain", IEEE Trans. Antennas and Propagat., vol. AP-34, pp. 1013-1023, Aug. 1986.

[16] R. J. Luebbers, "Finite conductivity uniform GTD versus knife edge diffraction in orediction of propagation path loss", IEEE Trans. Antennas and Propagat., vol. AP-32, pp. 70-76, Jan. 1984.

[17] R. Janaswamy, "A Fredholm integral equation method for propagation over small terrain irregularities", IEEE Trans. Antennas and Propagat., vol. 40, pp. 1416-1422, Sept. 1994. 
[18] J. T. Hviid, J. B. Anderson, J. Toftgard, and J. Bojer, "Terrain based propagation model for rural area - an integral equations approach", IEEE Trans. Antennas and Propagat., vol. 43, pp. 41-46, Jan. 1995.

[19] J. T. Johnson, R. T. Shin, J. C. Eidson, L. Tsang, and J. A. Kong, "A method of moments model for VHF propagation", IEEE Trans. Antennas and Propagat., vol. 45, pp. 115-125, Jan. 1997.

[20] C. Brennan and P. J. Cullen, "Application of the fast far-field approximation to the computation of UHF pathloss over irregular terrain", IEEE Trans. Antennas and Propagat., vol. 46, pp. 881-890, June 1998.

[21] J. A. López, M. R. Pino, F. Obelleiro and J. L. Rodríguez, "Application of the spectral acceleration forward-backward method to coverage analysis over terrain profiles", J. of Electromagn. Waves and Appl., vol. 15, pp. 10491074, Aug. 2001.

[22] D. Holliday, L. L. DeRaad Jr., and G. J. St.-Cyr, "Forward-Backward method for scattering from imperfect conductors", IEEE Trans. Antennas and Propagat., vol. 46, pp. 101-107, Jan. 1998.

[23] J. V. Toporkov, R. T. Marchand, and G. S. Brown, "On the discretization of the integral equation describing scattering by rough conducting surfaces", IEEE Trans. Antennas and Propagat., vol. 46, pp. 150-161, Jan. 1998.

[24] R. J. Adams and G. S. Brown, "A combined field approach to scattering from infinite cylinders using the method of ordered multiple interactions ", IEEE Trans. Antennas and Propagat., vol. 47, pp. 364-375, Feb. 1999.

[25] J. C. West and J. M. Sturm, "On iterative approaches for electromagnetic raough-surface scattering problems ", IEEE Trans. Antennas and Propagat., vol. 47, pp. 1281-1288, Aug. 1999.

[26] M. R. Pino, L. Landesa, J. L. Rodríguez, and F. Obelleiro, "The generalized forward-backward method for analyzing the scattering from targets on 
ocean-like rough surfaces", IEEE Trans. Antennas and Propagat., vol. 47, pp. 961-969, June 1999.

[27] H.-T. Chou and J. T. Johnson, "Formulation of forward-backward method using novel spectral acceleration for the modeling of scattering from impedance rough surface", IEEE Trans. Geosci. Remote Sensing, vol. 38, pp. 605-607, Jan. 2000.

[28] J. C. Wang, H.-T. Chou, Y.-Y. Kuo, and H.-Y. Chen, "An effective numerical modeling for the high frequency radio wave propagation over the forest canopies", IEEE Geoscience and Remote Sensing International Symposium, vol. 7, pp. 3114-3116, July 2000.

[29] H.-T. Chou, " Applications of forward-backward method in the fast analysis of large array problems", IEEE Antennas and Propagation Society International Symposium, vol. 4, pp. 2336-2339, July 2000.

[30] H.-T. Chou and H.-K. Ho, " A generalized forward-backward method for the efficient analysis of large array problems", IEEE Antennas and Propagation Society International Symposium, vol. 3, pp. 40-43, July 2001.

[31] D. Torrungrueng and J. T. Johnson, "Some issues related to the novel spectral acceleration method for the fast computation of radiation/scattering from one-dimensional extremely large scale quasi-planar structures", Radio Sci., vol. 37, No. 2, Mar. 2002.

[32] M. R. Pino, R. J. Burkholder, and F. Obelleiro, "Spectral acceleration of the generalized forward-backward method", IEEE Trans. Antennas and Propagat., vol. 50, pp. 785-797, June 2002.

[33] Ö. A. Çivi, "Extension of forward backward method with DFT based acceleration algorithm for the efficient analysis of large printed dipole arrays", IEEE Antennas and Propagation Society International Symposium, vol. 2, pp. 626-629, July 2002. 
[34] T. B. A. Senior, "Impedance boundary conditions for imperfectly conducting surfaces", Appl. Sci. Res., vol. 8, pp. 418-436, 1961.

[35] K. M. Mitzner, "An integral equation approach to scattering from a body of finite conductivity", Radio Sci. Res., vol. 2, pp. 1459-1570, 1967.

[36] D.-S. Wang, "Limits and validity of the impedance boudary condition on penetrable surfaces", IEEE Trans. Antennas and Propagat., vol. AP-35, pp. 453-457, Apr. 1987.

[37] S.-W. Lee and W. Gee, "How good is the impedance boundary condition?", IEEE Trans. Antennas and Propagat., vol. 50, pp. 1313-1315, Nov. 1987.

[38] R. Mittra and A. Peterson, Numerical techniques for RCS computation and scattering center approach to RCS modeling, Lecture Note, vol. 2

[39] C. A. Balanis, Advanced Engineering Electromagnetics, John Wiley \& Sons, 1989.

[40] C. D. Moss, T. M. Grzegorczyk, H. C. Han, J. A. Kong. "Scattering from layered rough surfaces using the forward backward method with spectral acceleration", to be published PIERS 2003.

[41] D. Torrungrueng and J. T. Johnson, "Numerical studies of back-scattering enhancement of electromagnetic waves from two-dimensional random rough surfaces using the FB/NSA method", J. Opt. Soc. Am. A Opt. Image Sci., vol. 18 , pp. 2518-2526, 2001

[42] D. Torrungrueng and J. T. Johnson, "The forward-backward method with a novel acceleration algorithm for the computation of scattering from twodimensional large-scale impedance random rough surfaces", Microwave Opt. Technol. Lett., vol. 29, 232-236, 2001.

[43] Y. Okumura, E. Ohmori, T. Kawano, and K. Fukuda, "Field Strength and Its Variability in VHF and UHF Land-Mobile Radio Service", Review of 
the Electrical Communication Laboratory, vol. 16, pp. 825-873, SeptemberOctober, 1968.

[44] M. Hata, "Empirical Formula for Propagation Loss in Land Mobile Radio Services", IEEE Transactions on Vehicular Technology, vol. VT-29, no. 3, August 1980.

[45] "Calculation of free-space attenuation", Recommendation ITU-R PN.525-2, 1994.

[46] "Propagation by diffraction", Recommendation ITU-R PN.526-7, 2001.

[47] J. Epstein and D. Peterson, "An experimental study of wave propagation at 850 MHz.", Proceedings of IEEE, vol. 41, no. 5, pp. 595-611

[48] K. Bullington, "Radio Propagation at frequencies above 30 megacycles" Proc. IRE, vol. 35, pp. 1122-1136, October 1947.

[49] J. Deygout, "Multiple knife-edge diffraction of microwaves", IEEE Trans. Antemas Propagat., vol. AP-14, no. 4, pp. 480-489, July 1966. 